\title{
Role of Extracellular Vesicles in Neuroinflammatory Progression and Mitochondrial Functional Alterations
}

\author{
Ashley E. Russell \\ West Virginia University, aekerr@mix.wvu.edu
}

Follow this and additional works at: https://researchrepository.wvu.edu/etd

Part of the Molecular and Cellular Neuroscience Commons

\section{Recommended Citation}

Russell, Ashley E., "Role of Extracellular Vesicles in Neuroinflammatory Progression and Mitochondrial Functional Alterations" (2019). Graduate Theses, Dissertations, and Problem Reports. 3947.

https://researchrepository.wvu.edu/etd/3947

This Dissertation is protected by copyright and/or related rights. It has been brought to you by the The Research Repository @ WVU with permission from the rights-holder(s). You are free to use this Dissertation in any way that is permitted by the copyright and related rights legislation that applies to your use. For other uses you must obtain permission from the rights-holder(s) directly, unless additional rights are indicated by a Creative Commons license in the record and/ or on the work itself. This Dissertation has been accepted for inclusion in WVU Graduate Theses, Dissertations, and Problem Reports collection by an authorized administrator of The Research Repository @ WVU.

For more information, please contact researchrepository@mail.wvu.edu. 


\title{
Role of Extracellular Vesicles in Neuroinflammatory Progression and Mitochondrial Functional Alterations
}

\author{
Ashley E. Russell
}

A dissertation submitted to the West Virginia University Health Science Center Neuroscience Program in partial fulfillment of the requirements for the degree of:

Doctor of Philosophy in Neuroscience

\author{
James W. Simpkins, Ph.D., Chair \\ Candice M. Brown, Ph.D. \\ Werner J. Geldenhuys, Ph.D. \\ Rosana Schafer, Ph.D. \\ Gregory Konat, Ph.D. \\ WVU Neuroscience Program \\ Morgantown, West Virginia \\ 2019
}

Keywords: Neuroinflammation, microRNAs, Extracellular Vesicles, Mitochondria

Copyright 2019 Ashley E. Russell 


\title{
ABSTRACT \\ Role of Extracellular Vesicles in Neuroinflammatory Progression and Mitochondrial Functional Alterations
}

\begin{abstract}
Ashley E. Russell
Inflammation within the central nervous system (CNS), termed neuroinflammation, is a defining characteristic of many neuropathological conditions, including Alzheimer's disease (AD) and stroke. Certain inflammatory mediators activate the transcription factor $\mathrm{NF}-\kappa \mathrm{B}$, which induces transcription of many pro-inflammatory genes, including miR-34a and miR-146a. Several target candidate genes of these miRNAs encode for proteins of the mitochondrial electron transport chain. In our studies, we demonstrate that in response to inflammatory stimuli, such as TNF- $\alpha$, the expression of miR-34a and -146a is significantly increased in several CNS cell types, and in their secreted extracellular vesicles (EVs). Exposure to TNF- $\alpha$-derived EVs significantly increases cellular bioenergetics in naïve recipient cells, with a concurrent increase in reactive oxygen species, indicative of impaired mitochondrial function. Further, using an animal model of experimental transient middle cerebral artery occlusion, we assess the effects of intermittent systemic LPS exposure, with long recovery periods, on stroke infarct volume. Relative to saline controls, animals repeatedly exposed to LPS have significantly larger cortical infarcts, and lower mRNA expression of autophagy genes. Inflammation-induced reduction in autophagy may lead to post-stroke increases in apoptosis. Together, these data suggest that the modulation of miR146a and miR-34a in response to neuroinflammatory stimuli may mediate the loss of mitochondrial integrity and function of cells, and that EVs significantly impair mitochondrial function in recipient cells. Further, intermittent systemic inflammation significantly alters the neuroinflammatory response within the brain, leading to increased stroke infarct volumes.
\end{abstract}




\section{Dedication}

This dissertation is dedicated to my parents, Michael and Sharri Kerr. Your unconditional love and support have been instrumental in shaping me into the person I am today. You taught me to always try my best and never give up. I know that wherever life takes me, you will always be right there with me. I love you.

This dissertation is dedicated to my late grandmother, Jeanette Will. You had such a substantial impact on my life in ways that you probably never realized. You instilled in me a passion for learning, which I have carried with me to this day. I am so proud to have called you my grandmother.

This dissertation is dedicated to my husband, Cory Russell. Words cannot describe the amount of appreciation and love I have for you. You are my rock. You have kept me sane over the past few years, and I honestly do not know if I could have made it through graduate school without you by my side. Thank you for loving me, caring for me, and making me laugh every single day. 


\section{Acknowledgments}

I would like to thank my mentor and advisor, Dr. James W. Simpkins. Thank you for taking me in and giving me a chance when I was just a lost first-year rotation student with no laboratory to call home. Your guidance, mentorship, and support have helped me become the scientist I am today. Thank you for letting me pursue my scientific passion.

I would also like to thank my committee members, Dr. Candice M. Brown, Dr. Werner J.

Geldenhuys, Dr. Rosana Schafer, and Dr. Gregory Konat for your support, guidance, and insight, which have allowed me to succeed.

Lastly, I would like to thank the current and past members of the Simpkins' lab, with a special thanks to Dominic Quintana, Stephanie Rellick, Saumyendra Sarkar, John Cavendish, Sujung Jun, Keyana Porter, and Sara Lewis. Your friendships made this lab feel like home. :) 


\section{Table of Contents}

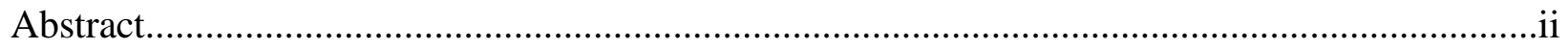

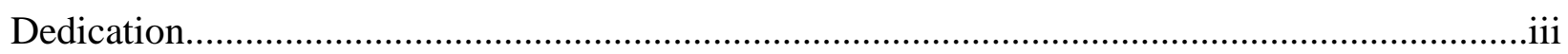

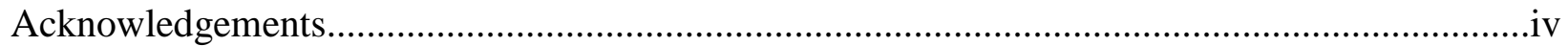

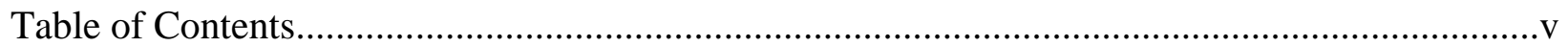

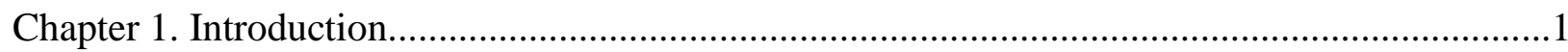

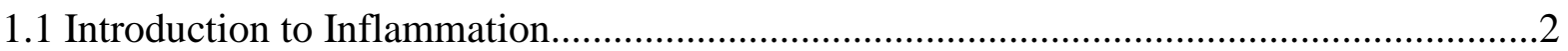

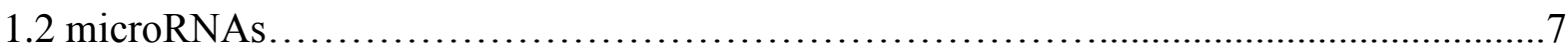

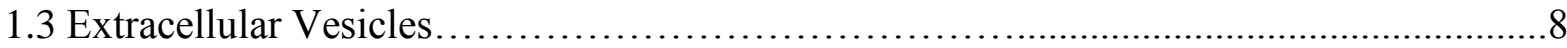

1.4 Neuroinflammation...............................................................

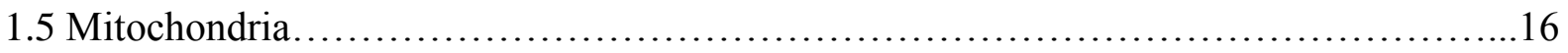

1.6 Alzheimer's Disease.........................................................

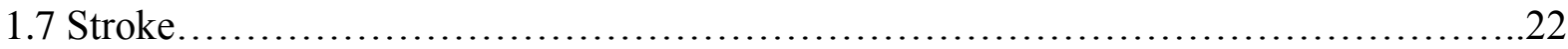

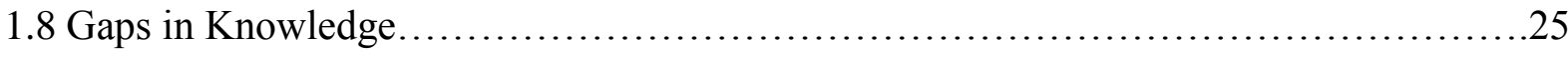

Chapter 2. miR-146a dysregulates energy metabolism during neuroinflammation....................27

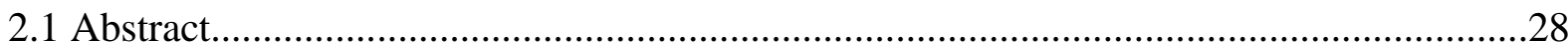

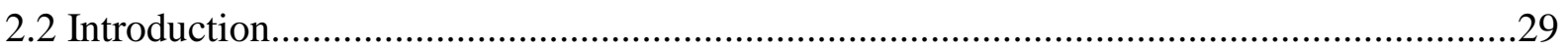

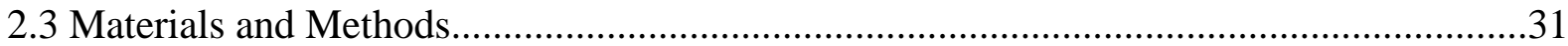

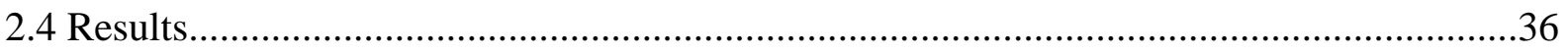

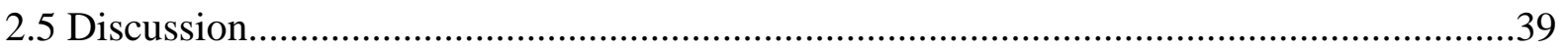

Chapter 3. Extracellular vesicles secreted in response to cytokine exposure increase

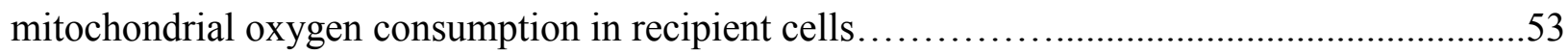

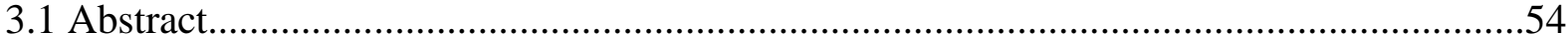

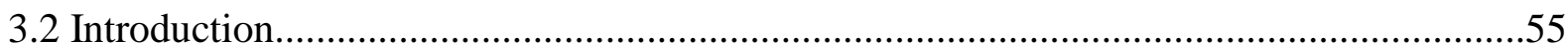

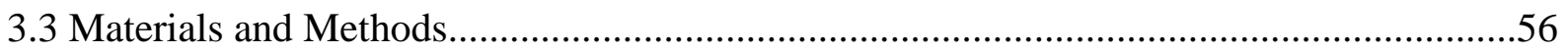

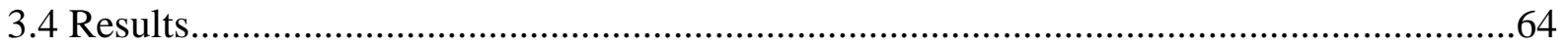


3.5 Discussion..

Chapter 4. Intermittent lipopolysaccharide exposure significantly increases cortical infarct

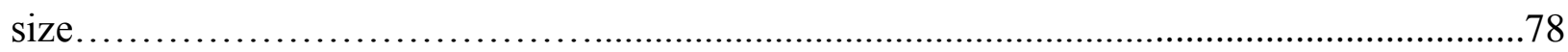

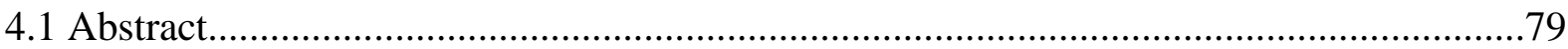

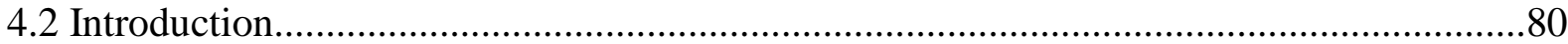

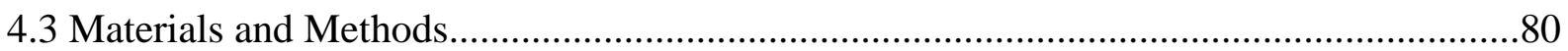

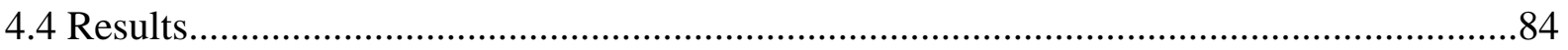

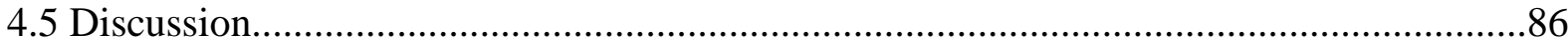

\section{Chapter 5.}

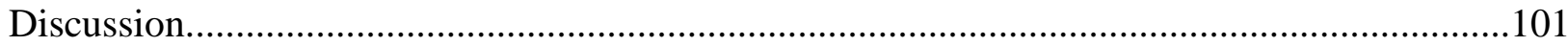

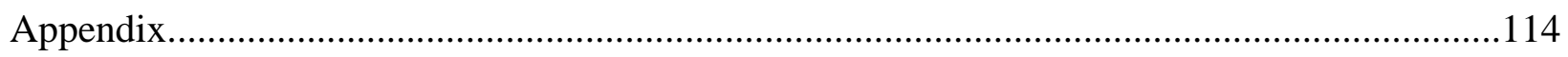

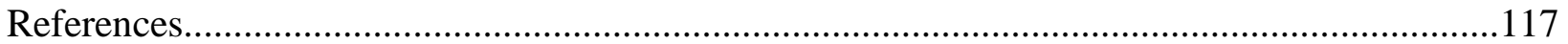




\section{Figure and Tables}

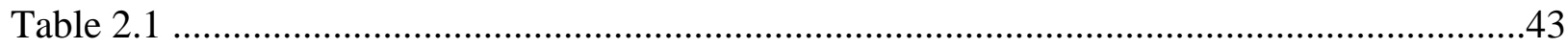

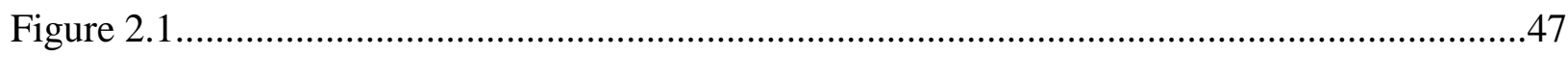

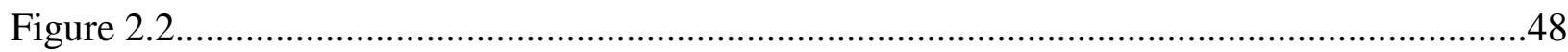

Figure 2.3

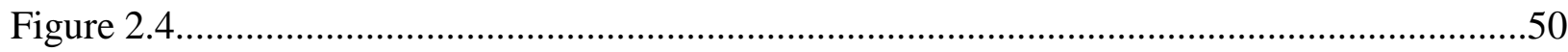

Figure 2.5

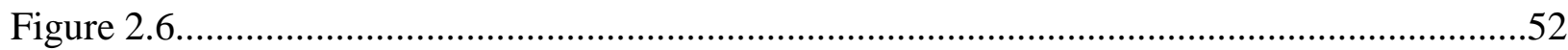

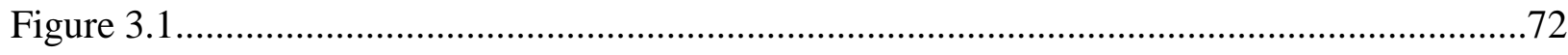

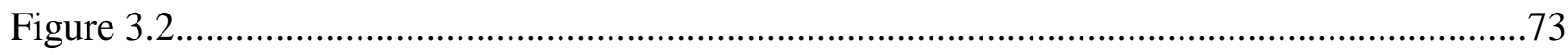

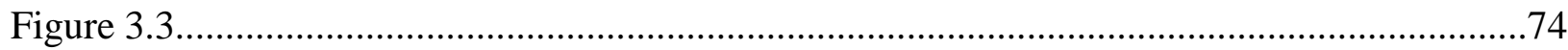

Figure 3.4

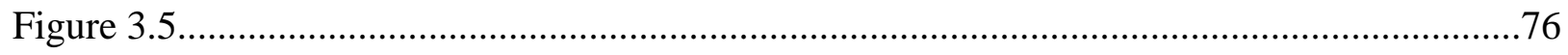

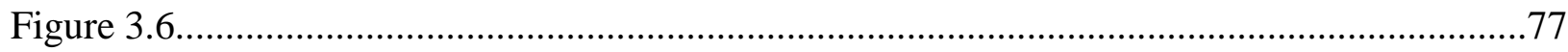

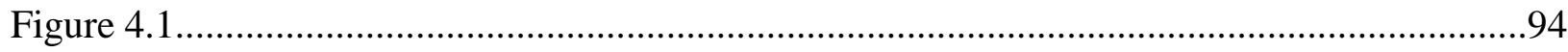

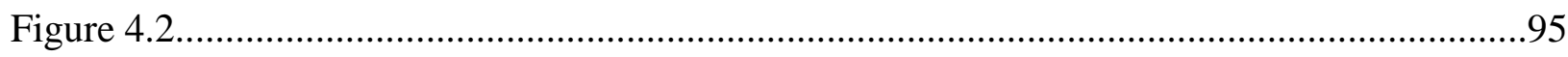

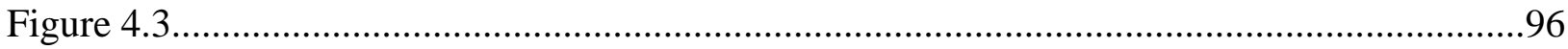

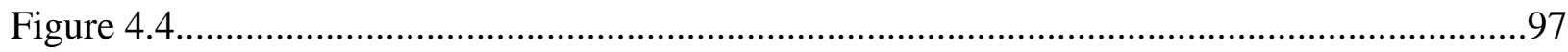

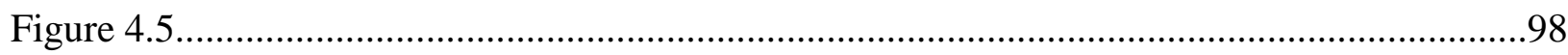

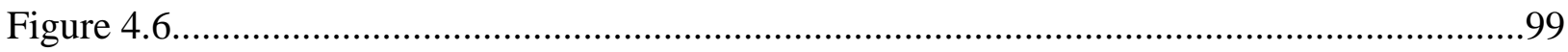

Figure 4.7

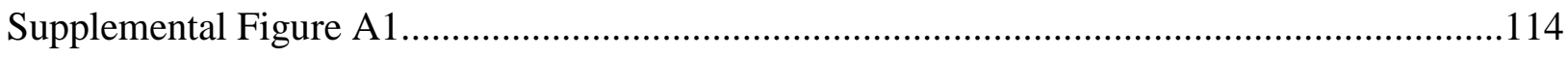

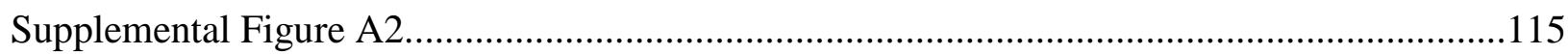

Supplemental Figure A3 …………………………………………………………...116 
Chapter 1

\section{Introduction}




\subsection{Introduction to Inflammation}

Inflammation is an evolutionary adaptation designed to protect organisms from pathogenic infections, remove and restore damaged tissue after injury, and eliminate mutated cells.

Organisms must be able to regulate and balance the immune response to allow for the clearance of pathogens and damaged tissue, while restricting the death of healthy cells. Unfortunately, the immune system is not perfect and a multitude of things can go awry, giving rise to ailments such as autoimmune diseases, cancers, and chronic inflammation.

The immune system is subdivided into two arms comprised of the innate and adaptive systems. While the adaptive branch of the system allows for highly specific recognition of non-self antigens, the innate arm operates on a more general level of detection, surveying the environment for highly conserved structural motifs present on many pathogenic invaders. This is due to the expression of pattern recognition receptors (PRRs) on innate immune cells, which can detect both pathogen associated molecular patterns (PAMPs) expressed on microorganisms like bacteria, and damage associated molecular patterns (DAMPs), which are altered or damaged endogenous ligands, usually released by necrotic, senescent, and/or damaged cells. Among PRRs, toll-like receptors (TLRs) play a central role in the progression of inflammation (Kielian, 2006; Takeda et al., 2003) since their engagement triggers a potent immune signaling pathway; activation of the family of transcription factors, nuclear factor kappa-light-chain-enhancer of activated B cells (NF-kB) (Oeckinghaus and Ghosh, 2009; Kawasaki and Kawai, 2014).

\section{A. NF-kB signaling}

Initially discovered in the Drosophila, TLRs participate in the immune response by recognizing specific molecular patterns. To date, 10 human and 12 murine TLRs have been described, each of which recognizes and responds to different molecular patterns (Kawasaki and Kawai, 2014). For example, TLR4 recognizes lipopolysaccharide (LPS; also referred to as endotoxin), which is one of the main constituents of Gram-negative bacterial cell walls, while TLR3 recognizes double-stranded RNA (dsRNA), characteristic of viruses. Some TLRs are expressed on the cell surface (e.g. TLR4) and participate in identifying microbial membrane components, while other 
TLRs (e.g. TLR3) are expressed on intracellular compartments, such as endosomes, and mostly recognize microbial or viral nucleic acids (Kawai and Akira, 2010). Upon their activation, an intracellular signaling cascade is initiated, eventually leading to the activation of NF- $\mathrm{BB}$.

The NF- $\kappa \mathrm{B}$ complex refers to a family of five Rel proteins expressed in nearly all cell types and tissues. These proteins can homo- or heterodimerize, and upon activation, translocate to the nucleus to bind with specific promotor/enhancer regions of DNA; thus NF- $\kappa$ B plays a prominent role in altering gene transcription in response to various stimuli (Oeckinghaus and Ghosh, 2009). The two major pathways by which NF- $\kappa \mathrm{B}$ can be activated are the canonical, and the alternative (noncanonical) pathways. TLRs or tumor necrosis factor-alpha receptor (TNFR) activation can activate the canonical pathway. Briefly, within the cytoplasm, the NF- $\kappa \mathrm{B}$ complex is sequestered by the inhibitor of $\kappa \mathrm{B}(\mathrm{I} \kappa \mathrm{B})$, which prevents the complex from exerting any transcriptional effects. Activation of the canonical pathway initiates the inducible degradation of I $\mathrm{B}$ via its phosphorylation by IкB kinase (IKK), and thus targeting it for ubiquitination and eventual degradation by the proteasome (Karin and Delhase, 2000). NF- $\kappa B$ is subsequently translocated to the nucleus and activates the transcription of dozens of genes, whose functions range from cellproliferation and anti-apoptotic mechanisms, to transcription of immunomodulators, such as chemokines and cytokines, including tumor necrosis factor-alpha (TNF- $\alpha$ ) (Oeckinghaus and Ghosh, 2009; Pahl, 1999).

\section{B. TNF- $\alpha$ Synthesis and Signaling}

Carswell and colleagues identified a serum factor that induced tumor necrosis, later termed TNF$\alpha$ (Carswell et al., 1975). After decades of research, it has been established that TNF- $\alpha$ is responsible for more than just initiating tumor cell death; its signaling is a highly complex

process that plays a key role in the immune response. Interestingly, TNF- $\alpha$ is produced in response to NF- $\kappa \mathrm{B}$ activation, and its signaling also activates $\mathrm{NF}-\kappa \mathrm{B}$; as such, the proinflammatory cytokine $\mathrm{TNF}-\alpha$ is a major player in the progression of inflammation. 
TNF- $\alpha$ is a member of the TNF superfamily, which includes 19 ligands, all of which signal through at least one of the 29 TNF-receptors (TNFRs) (MacEwan, 2002; Aggarwal, 2003; Wajant et al., 2003; Montgomery and Bowers, 2012). The ligands of the TNF superfamily are capable of inducing cellular proliferation, differentiation, survival, and apoptosis (Aggarwal, 2003). TNF- $\alpha$ is produced by a variety of cell types including most immune cells, endothelial cells, microglia, astrocytes, and neurons (Montgomery and Bowers, 2012), and signals through TNFR1 expressed on all nucleated cells, and TNFR2, expressed on immune, endothelial, and nervous system cells (MacEwan, 2002; Aggarwal, 2003; Montgomery and Bowers, 2012).

TNF- $\alpha$ expression is regulated at the transcriptional, post-transcriptional, and translational level, dependent upon stimulation by pathogens, injury, and immune activation (Montgomery and Bowers, 2012). TNF- $\alpha$ mRNA translation results in a $26-\mathrm{kDa}$ transmembrane-associated integral protein: tmTNF (also referred to as pro-TNF- $\alpha$ ), generally organized in stable homotrimers (MacEwan, 2002; Wajant et al., 2003; Montgomery and Bowers, 2012). TNF- $\alpha$ converting enzyme (TACE) can cleave tmTNF protein to a 51-kDa soluble trimeric form, sTNF, better known as TNF- $\alpha$ (MacEwan, 2002; Wajant et al., 2003; Montgomery and Bowers, 2012).

Understanding the difference between the two forms of TNF (tmTNF and sTNF) and how they exert their signaling pathways is of utmost importance. sTNF has a high affinity for TNFR1 and a slow dissociation period, while tmTNF has a high affinity for TNFR2 (MacEwan, 2002; Montgomery and Bowers, 2012). TNFR1 and TNFR2 are not enzymatically active themselves. These receptors must form homotrimers and associate with intracellular adaptor proteins referred to as TNF receptor-association factors (TRAFs), to induce intracellular signaling pathways upon receptor activation; TRAFs are signal transducers for TNF- $\alpha$ and its receptors (TNFR1 \& TNFR2) (MacEwan, 2002).

Signaling through TNFR1 leads to the formation of two complexes, which lead to the activation of NF- $\kappa \mathrm{B}$ or apoptosis, respectively (Micheau and Tschopp, 2003). On the cytoplasmic portion of the TNFR1 is a death domain (DD), which is usually blocked by the silencer of death domain (SODD) protein. When the receptor is activated by binding of TNF- $\alpha$, within seconds SODD 
dissociates and allows TRADD (TNF receptor-associated death domain) and receptor interacting serine/threonine kinase 1 (RIP1) to interact with the DD (MacEwan, 2002). TRAF2/5 indirectly interacts with TNFR1 by associating with TRADD. This signaling complex of proteins is referred to as complex I, and its formation leads to activation of NFאB (Micheau and Tschopp, 2003). TRAF2/5 activates NFkB-inducing kinase (NIK) which phosphorylates the inhibitor of $\kappa \mathrm{B}$ kinase (IKK), which then leads to the activation of NFאB (MacEwan, 2002) as described in the previous section. As activation of NF- $\kappa \mathrm{B}$ can lead to the transcription of anti-apoptotic genes (e.g. the caspase-8/10 inhibitor, cFLIP), signaling through formation of complex I only does not result in the activation of apoptosis (Ting and Bertrand, 2016). Formation of complex II occurs more transiently, as it requires dissociation of TRADD from complex I and subsequent association with Fas associated via death domain (FADD) (Ting and Bertrand, 2016). This complex recruits and activates caspase-8/10, which successively activates caspase-3 and leads to initiation of apoptosis (MacEwan, 2002; Micheau and Tschopp, 2003).

TNFR2 lacks the DD associated with TNFR1 and has the capacity to directly interact with TRAF2 under some conditions (MacEwan, 2002). This interaction allows for the recruitment of receptor-interacting protein (RIP) and Fas-associated death domain (FADD), which contains a death effector domain (DED) (MacEwan, 2002). This complex can associate with, and activate caspases (namely caspase-8) and lead to cellular apoptosis, or activation of NF- $\kappa \mathrm{B}$, leading to alterations in gene transcription (MacEwan, 2002). Other signaling pathways associated with TNFR2 are not yet fully understood; more is known about TNFR1 pathway activation.

TNF- $\alpha$ is a key regulator of the immune response, low levels of TNF- $\alpha$ promotes the swift initiation of defense responses to inflammatory stimuli, however high levels of TNF- $\alpha$ can lead to chronic inflammation and tissue damage (Tracey et al., 2008). Aberrant signaling of this molecule has been implicated in many systemic inflammatory diseases, such as Crohn's and various types of arthritis (Bradley, 2008; Tracey et al., 2008), and has also been shown to be upregulated in the brains of patients suffering from neuroinflammatory diseases (Montgomery and Bowers, 2012). 
Aside from its role in inflammation, TNF- $\alpha$ has also been implicated in neuronal proliferation, even into adulthood. Adult neurogenesis occurs within only two regions of the brain; the subventricular zone (SVZ) lining the lateral ventricles and the subgranular zone (SGZ) of the dentate gyrus (which is part of the hippocampus thought to be responsible for the formation of episodic memories). Injury and inflammation can modulate the formation of new cells within these regions which can migrate to the site of injury (Wu et al., 2000; Heldmann et al., 2005; Widera et al., 2006). These proliferating progenitor cells express TNF- $\alpha$ and its receptors (Bernardino et al., 2008; Keohane et al., 2010), raising the possibility that in these cells TNF- $\alpha$ signaling is occurring in both paracrine and autocrine manners (Heldmann et al., 2005). The role of TNF- $\alpha$ in this process however, remains yet to be fully elucidated as various labs report conflicting data. Some reports suggest that exogenous administration of TNF- $\alpha$ induces cellular proliferation within the rat SVZ (Wu et al., 2000; Bernardino et al., 2008), while others find that TNF- $\alpha$ administration reduces progenitor cell number, alters neuron morphology, and increases astrocytes and glia (Neumann et al., 2002; Monje et al., 2003; Golan et al., 2004; Liu et al., 2005; Keohane et al., 2010). Inhibition of adult hippocampal neurogenesis has negative consequences on hippocampal-dependent tasks, such as learning temporal associations, suggesting the formation of these types of associations and memories require the development and integration of newly born neurons (Shors et al., 2001). Patients suffering from Alzheimer's disease (AD) struggle with forming new memories and correctly recalling old ones, both of which are hippocampal dependent functions. As will be discussed in a later section, AD is considered a chronic neuroinflammatory disease, and levels of TNF- $\alpha$ are upregulated in patient brains (Sharma et al., 2012), thus furthering the possibility that TNF- $\alpha$ negatively influences adult neurogenesis.

TNF- $\alpha$ is just one of many cytokines critically involved in the initiation and progression of the immune response. Activation of pathways that alter gene transcription, like NF- $\kappa B$, play major roles in the complex process of inflammatory initiation, progression, and resolution. At the posttranscriptional level, other regulators such as microRNAs can come into play and alter inflammatory signaling and disease status. 


\section{2 microRNAs}

MicroRNAs (miRNAs) are small ( 22 nucleotides), non-coding RNAs that play important regulatory roles as gene repressors (Chen and Rajewsky, 2007; Ha and Kim, 2014; Kim, 2005). They function by base pairing to complementary sites within the 3' untranslated region (UTR) of mRNAs, which inhibits translation or leads to the decapping of the 5' mRNA, thus targeting the mRNA for degradation (Rehwinkel et al., 2005; Ha and Kim, 2014). Perfect miRNA basepairing with mRNA is not necessary; candidate target sites for miRNA-mRNA interaction require $~ 6-8$ nucleotides in the 5' region of the miRNA and the 3' UTR of the mRNA to pair (Doench and Sharp, 2004; Krek et al., 2005). Because of this, miRNAs can be a tad promiscuous in their inhibitory functioning; a single miRNA can target approximately 200 mRNAs (Krek et al., 2005). Similarly, a single mRNA can be targeted by several miRNAs, and it is thought that approximately $30 \%$ of human genes can be regulated by miRNAs (Lewis et al., 2005).

RNA polymerase II or III (Pol II/III) transcribe miRNA genes as long primary transcripts $(\sim 1 \mathrm{~kb})$ termed pri-miRNAs; these contain a hairpin structure in which the mature miRNA sequence resides (Ha and Kim, 2014). Within the nucleus, Drosha and DGCR8 form a microprocessor COMPLEX, which cleaves the hairpin structure ( 65 nucleotides), giving rise to a pre-miRNA (Ha and Kim, 2014). Exportin 5 assists with the translocation of the pre-miRNA into the cytoplasm, where Dicer cleaves the pre-miRNA near the terminal loop, creating a doublestranded RNA molecule (Ha and Kim, 2014). Next, the guide strand of the RNA duplex is preferentially loaded onto an Argonaute (AGO) protein, and the passenger strand of RNA (miRNA*) is removed, allowing for the formation of a mature RNA-induced silencing complex (RISC) (Ha and Kim, 2014). The RISC is now capable of targeting complementary mRNAs; near perfect complementarity generally results in RNA slicing (Tolia and Joshua-Tor, 2007), while imperfect complementarity results in translational repression via the blockage of proteinprotein interactions (Iwasaki et al., 2009) or by decapping the mRNA, followed by subsequent degradation (Rehwinkel et al., 2005; Behm-Ansmant et al., 2006).

miRNAs are involved in many important cellular functions, and alterations in their expression are associated with numerous diseases by altering protein expression. A mounting body of 
evidence documents a large number of miRNAs are involved in regulating inflammation. For example, in normal physiological conditions, transcription of miR-146a, miR-155, and miR-21 remain at low levels. However activation of TLRs results in strong NF- $\kappa B$-dependent induction and subsequent upregulation of these miRNAs (Taganov et al., 2006; Olivieri et al., 2013; Rippo et al., 2014). Further, several components of TLR signaling are targeted by these miRNAs, suggesting their complex role in regulating the inflammatory cascade (Quinn and O'Neill, 2011).

\subsection{Extracellular Vesicles}

An interesting point of discussion is that miRNAs are not confined to the cell IN which they were transcribed; they can be secreted out of one cell and taken up by another. This mechanism is usually mediated by extracellular vesicles (EVs). The study of EVs is a newly emerging, exciting area of research, and over the past three and a half decades, the field has progressed substantially. The first description of EVs occurred in the early 1980's; within a week of one another, two seminal papers from independent labs were published, describing the release of transferrin receptors from maturing reticulocytes via externalized vesicles (Harding and Stahl, 1983; Pan and Johnstone, 1983). The exosome release pathway (which will be described in the following section) was first described in the same year (Harding et al., 1983, 1984). EVs were originally thought of "garbage bags" by which cells packaged and disposed of unwanted materials (e.g. unnecessary transferrin receptors) (Pan et al., 1985; Johnstone et al., 1987). With time, and increasing interest from other research areas, EVs began to be viewed as more than just waste disposal units. Today, scientists from virtually every area of biological research are becoming involved in the study of EVs, with cancer, immunology, and neuroscience being some of the largest areas of ongoing EV research.

\section{A. What Are EVs?}

EVs are small, nanosized $(<2 \mu \mathrm{m})$, membrane-bound vesicles shed (or secreted) by most, if not all cell types into the extracellular environment. The term "EV" generally refers to three distinct subpopulations of vesicles, each with specific membrane compositions and unique biogenesis 
pathways. Apoptotic bodies are formed by cells undergoing apoptosis; these vesicles are large $(500-2,000 \mathrm{~nm})$ and express high amounts of phospatidyl-serine, which can act as an "eat me" signal to macrophages (Lee et al., 2012a; Segawa and Nagata, 2015), and will not be discussed in further detail. Microvesicles (MVs; sometimes referred to as ectosomes) are shed from the outward budding of the plasma membrane. These vesicles are slightly smaller in size (100 1,000 nm), and express integrins, selectins, and CD40 ligands (Lee et al., 2012a; Raposo and Stoorvogel, 2013). Lastly, exosomes, which are the smallest type of EV (40 - $100 \mathrm{~nm})$, are derived from the endosomal pathway.

Biogenesis of exosomes is much more complicated than that of apoptotic bodies or MVs. Briefly, as an early endosome matures to a late endosome, its membrane begins to bud inwards, creating intraluminal vesicles (ILVs) in the process, and transforming into what is now considered a multivesicular body (MVB) (Stoorvogel et al., 1991). MVBs can fuse with lysosomes and their contents are degraded. Alternatively, MVBs can fuse with the cell's plasma membrane, thus releasing their ILVs into the extracellular environment, giving rise to exosomes. As these vesicles undergo several processing steps, they express specific proteins which assist in the formation of multivesicular bodies (e.g. ALIX, TSG101), proteins involved in membrane transport and fusion (annexins, flotillin, Rab proteins), and the canonical exosome proteins CD63, CD81, CD9 (Lee et al., 2012). It is now thought that prior to packaging ILVs, late endosomes are destined for one fate or another, and that cells can host different subpopulations of MVBs (White et al., 2006). This however, depends on several factors including their location within the cell (Buschow et al., 2005), the stimulatory factor inducing fusion with lysosomes or plasma membrane, (Wubbolts et al., 2003), and where in the maturation processes a cell is (Colombo et al., 2014).

EVs function as cell-to-cell communicators, transferring information from one cell to another. Indeed, the RNA and protein cargo within EVs is functional and can exert effects on recipient cells. The first evidence of the horizontal transfer of proteins via extracellular vesicles was found nearly two decades ago (Mack et al., 2000; Baj-Krzyworzeka et al., 2002; Rozmyslowicz et al., 2003; Janowska-Wieczorek et al., 2005). Not long after, EV-mediated horizontal transfer of 
mRNA and miRNA was demonstrated (Valadi et al., 2007). Transferred mRNAs could be translated into proteins, and miRNAs were able to function as translational repressors (Lotvall and Valadi, 2007; Colombo et al., 2014).

It is thought that while both exosomes and MVs contain functional proteins and RNAs of the parent cell from which they are derived, exosomal cargo has been specifically, and deliberately, packaged for secretion from the cell (McKenzie et al., 2016). Indeed, there is some evidence suggesting that there are highly specific sorting pathways for the packaging of exosomal cargo (Ramachandran and Palanisamy, 2012). The endosomal sorting complex required for transport (ESCRT) consists of four complexes (ESCRT-0, -I, -II, and -III), and is comprised of a family of approximately thirty proteins (Hanson and Cashikar, 2012). Each of the four ESCRT complexes can play a role in selectively packaging proteins and RNAs into ILVs within the endosome. ESCRT complexes -0, -I, and -II directly bind to ubiquinated proteins, and load them into ILVs (Hislop and von Zastrow, 2011). In addition to sorting ILV contents, ESCRT complexes influence the budding and cleavage of the endosomal membrane, thus giving way to the formation of ILVs (Gupta and Pulliam, 2014). While the ESCRT complexes have a large role in ILV formation, MVBs containing ILVs are still formed, even in the absence/inactivation of ESCRT (Stuffers et al., 2009), indicating the presence of an ESCRT-independent cargo-sorting pathway (Trajkovic et al., 2007).

Areas of the endosomal limiting membrane that become enriched in membrane spanning proteins, known as tetraspanins are referred to as Tetraspanin-enriched membrane domains (TEMs). These areas are often required for proper fission/fusion of vesicles as they begin to bud inwards on the endosomal membrane, thus also contributing to the formation of ILVs (Hemler, 2008). CD63 is a tetraspanin that is highly abundant on ILVs, and has been shown to play a role in the sorting of some proteins (van Niel et al., 2011). Additionally, neutral sphingomyelinase (Trajkovic et al., 2008) and phospholipase D2 (Ghossoub et al., 2014) are both lipid metabolism enzymes which have been shown to induce inward budding of the late endosomal limiting membrane, thus creating ILVs. 
The small GTPases Rab27a and Rab27b also play critical roles in exosome formation and secretion (Ostrowski et al., 2010; Alexander et al., 2017). The Arrestin domain-containing protein 1 (ARRDC1) has been shown to significantly affect the biogenesis and secretion of exosomes, and also mediate the release of a subpopulation of MVs derived from the plasma membrane (Nabhan et al., 2012; Mackenzie et al., 2016; Wang and Lu, 2017; Anand et al., 2018). Several groups have shown highly variable expression patterns in cells and their cognate EVs, which may be due to specificity of cargo sorting (Batagov et al., 2011; Montecalvo et al., 2012; Cha et al., 2015; McKenzie et al., 2016; Hinger et al., 2018).

Alternatively, MV cargo is likely packaged in a more coincidental manner and is reflective of the parental cell's cytoplasmic components near the plasma membrane at the time of shedding. Indeed, the RNA profile of large vesicles reveals more similarity than small vesicles to the cell from which they are derived (Wei et al., 2017). This however, may be a result solely based on vesicle size, as larger vesicles may incorporate full-length mRNAs into their lumen more easily than smaller EVs.

\section{B. EVs and Inflammation}

Evidence also suggests a role of inflammation in EV biogenesis and secretion (Théry et al., 2009). In response to TNF- $\alpha$ or interferon-gamma (IFN- $\gamma$ ) exposure, EVs released from mesenchymal stem cells contained very different protein and RNA profiles, compared to unstimulated control cells (Harting et al., 2018). Similarly, after LPS exposure, dendritic cells released significantly more EVs compared to EV concentrations released from unstimulated cells (Obregon et al., 2006). Further, dsDNA-induced inflammation also increases EV release (Takahashi et al., 2017), and endosomes expressing activated TLRs may also lead to inducible EV release (Srinivasan et al., 2017). Another group has also demonstrated that EVs express major histocompatibility complex I (MHCI) and MHCII, along with surface antibodies; therefore it is expected that these EVs are directly participating in the immune response (Utsugi-Kobukai et al., 2003; Luketic et al., 2007; Saunderson et al., 2008; Bobrie et al., 2011; Chaput and Théry, 2011). Recently, it has been shown that EVs may be targeted to inflamed tissue (Balusu et al., 
2016). Together, these data demonstrate that inflammatory stimuli can alter EV cargo and number, and these vesicles can act as immunomodulatory signaling molecules. These findings are not restricted to the peripheral immune response as EVs have been shown to also participate in neuroinflammation.

\subsection{Neuroinflammation}

Inflammation within the brain, termed neuroinflammation, can stem from within the central nervous system (CNS) or be induce via systemic inflammation. Due to the selective permeability of the blood-brain barrier (BBB), the CNS was previously considered "immune privileged", however, the CNS is indeed vulnerable to systemically-derived bacterial, viral, and autoimmune insult, and accumulating evidence supports the notion of highly complex immune-nervous system interactions (Galea et al., 2007).

\section{A. The Role of the Blood-Brain-Barrier}

The vasculature in some areas in the CNS (circumventricular organs, ventricles, choroid plexus, and meninges) is characterized by large fenestrations between endothelial cells, similar to peripheral vasculature (Meisel et al., 2005). There is direct communication between the CNS and circulating blood in these regions (Dermietzel and Leibstein, 1978; Prat et al., 2001), and due to a high concentration of cytokine receptors, they are well equipped for sensing changes in peripherally circulating cytokine levels (Haddad et al., 2002). Consequently, immune responses induced here are similar to those that occur in the periphery (Galea, 2007).

Conversely, the vascular endothelial cells that form the circulatory network for the rest of the brain comprise the blood-brain barrier (BBB). These cells form and maintain tight junctions in response to factors secreted by glial cells (Prat et al., 2001; Alvarez et al., 2013). The outdated concept of CNS immune privilege stemmed from the belief that the blood-brain barrier (BBB) was completely impenetrable, and therefore allowed the CNS to remain unaffected by systemic inflammation. Contrary to this belief, conclusive evidence shows that while tight junctions are 
selectively permeable and provide somewhat of a physical barrier, the BBB does not serve as an impassable blockade from all peripherally circulating factors.

During normal physiological conditions, tight junctions between endothelial cells prevent the infiltration of unwanted cells, molecules, bacteria, and viruses into the CNS. It has been shown that inflammation, both peripherally- and CNS-derived, can weaken the BBB. Some evidence also suggests a potential role for miRNAs in the inflammation-induced weakening of the BBB. Peripherally circulating cytokines can induce upregulation of miR-155 in brain endothelial cells and lead to the disruption of tight junctions between cells (Lopez-Ramirez et al., 2014). Neuroinflammation induced by the accumulation of amyloid $\beta(\mathrm{A} \beta)$ plaques in the brain have been shown to reduce miR-107 expression in brain endothelial cells, and lead to disruption of the BBB, the effects of which can be reversed upon overexpression of miR-107 (Liu et al., 2016b). The weakening of the BBB reduces its ability to prevent infiltration of peripherally circulating factors, such as soluble, blood-derived plasma proteins (Veerhuis, 2011), monocytes (Nottet et al., 1996; Persidsky and Gendelman, 1997), leukocytes (Wekerle et al., 1986; Hickey et al., 1991; Bell et al., 1996), and pathogens into the CNS, (Montagne et al., 2015). Upon infiltration, these factors further contribute to neuroinflammation.

The concept of inflammaging posits that aging induces chronic, low-grade systemic inflammation in the absence of overt infection, causing a shift in the immune system towards a pro-inflammatory state (Franceschi et al., 2018). In line with this, age has also been shown to heavily influence the permeability and functional capacity of the BBB (Prat et al., 2001; Zeevi et al., 2010; Montagne et al., 2015). Older adults and animals experience reduced transport of key metabolic molecules, such as glucose and choline, from the periphery into the CNS (Shah and Mooradian, 1997), which can lead to impaired neurological functioning. Just as in heightened inflammatory states, when the integrity of the BBB has been compromised with age, it becomes more permeable, or leaky, allowing peripheral substances entrance into the CNS (Shah and Mooradian, 1997). 
In order for immune cells to invade the CNS, they must first adhere to the brain endothelium; this is mediated by cellular adhesion molecules, namely ICAM-1 and VCAM-1 expressed on vascular ECs. In response to immunomodulatory factors that are increased during systemic inflammation such as TNF- $\alpha$, IFN- $\gamma$, and IL-1, brain microvascular ECs upregulate ICAM-1 and VCAM-1 by 3 - to 15 -fold, thus enhancing immune cell invasion. These cytokines may play a large role in the infiltration of peripherally circulating leukocytes into the CNS (Prat et al., 2001), while also contributing to progression of the neuroinflammatory response.

\section{B. The Role of Microglia}

When foreign, peripheral substances cross the $\mathrm{BBB}$, microglia (which are commonly referred to as the resident macrophages of the CNS) become activated. Under normal physiological conditions, microglia were long believed to be resting, or quiescent; however evidence gathered over the past decade indicates that microglia are constantly surveying the microenvironment for disturbances, such as protein aggregates, damaged neurons, alterations in extracellular potassium levels, or intruders like viruses, bacteria, or peripheral immune cells (Gehrmann et al., 1995; Davalos et al., 2005; Nimmerjahn et al., 2005; Morris et al., 2013). In response to inflammatory stimuli, astrocytes also become activated. These specialized cells respond by forming a physical barrier known as a glial scar, in an attempt to contain the inflammation and prevent it from spreading to neighboring, healthy tissue (Cekanaviciute and Buckwalter, 2016).

Microglia play a pivotal role in the neuroinflammatory response (González et al., 2014). When they become activated, they migrate towards the focal site of inflammation within minutes and phagocytose the inflammation-inducing substance (Davalos et al., 2005; Wu et al., 2007). Although resting-state microglia constitutively express immune regulatory markers, their activation leads to a rapid upregulation of these cellular immune markers, such as complement receptors and MHCII antigens, which allow them to interact with other cells in the CNS (e.g. other microglia, neurons, astrocytes, infiltrating T-cells) (Akiyama et al., 2000b). Additionally, activated microglia have also been shown to secrete cytokines, chemokines, complement factors, and free radicals, which promote inflammation and help direct the immune response (Perry et al., 
2001; Rock et al., 2004; Tuppo and Arias, 2005). In normal conditions, acute increases of inflammatory molecules can be beneficial as microglia remove damaged tissue or peripheral invaders; however, exaggerated or chronic activation of microglia can induce major pathology and neurodegeneration.

Inflammaging also appears to influence microglia and their capacity to react properly to brain insult. With age, microglia undergo a shift towards a pro-inflammatory state, and exhibit dysfunctional or exaggerated responses to inflammation, thus creating an unfavorable environment for neurons (Mosher and Wyss-Coray, 2014; Koellhoffer et al., 2017). Studies investigating the protein and RNA profiles of microglia in mice at different ages indicate that age and sex do appear to play a role in expression patterns, and that polarization towards the proinflammatory phenotype occurs at 12 months of age (Crain et al., 2013). Furthermore, several "hallmarks" of aging microglia have been described, including upregulation of TLRs, proinflammatory cytokines, and increased reactivity upon stimulation (Spittau, 2017).

Aside from traditional immune interactions described above, miRNAs have also been recently identified to play a role in microglial activation and regulation in response to inflammation (Guedes et al., 2013). Activation of microglia to either the "pro- inflammatory" M1 (classical activation) or "anti- inflammatory" M2 (alternative activation) phenotypes appears to be dependent on a network of miRNAs. For example, LPS exposure shifts microglia towards the M1 phenotype, downregulates miR-Let-7a expression, and subsequently leads to cell death (Cho et al., 2015). Overexpression of microglial miR-Let-7a altered the phenotypic activation to M2, reduced apoptosis and nitrite production, increased secretion of anti-inflammatory cytokines, and enhanced production of brain-derived neurotrophic factor (BNDF) (Cho et al., 2015). Interestingly, miR-124 is highly expressed in resting-state microglia but becomes significantly downregulated when microglia take on the M2 phenotype(Ponomarev et al., 2011). Overexpressing miR-124 in microglia prevents microglial activation from even occurring (Ponomarev et al., 2011). Furthermore, miR-155 is classically regarded as a pro-inflammatory $\mathrm{miR}$, and is upregulated in M1 activated microglia after exposure to cytokines like TNF- $\alpha$ and IFN- $\gamma$ (Cardoso et al., 2012). This has been shown to further enhance cytokine release from 
microglial cells; however, it has also been shown to induce IFN- $\beta$ secretion, which appears to activate an anti-inflammatory pathway (Dalpke et al., 2008; Wang et al., 2010). These data suggest that miR-155 may influence early induction of the inflammatory response, while also acting to regulate it downstream.

\section{EVs and Neuroinflammation}

Some of the first studies examining the relationship between EVs and the CNS occurred in the early 2000's (Greco et al., 2001; Marzesco et al., 2005; Fauré et al., 2006). Since then, several groups have shown that EVs participate in the spread of pathology within the brain (Vella et al., 2008), including the transfer of prions (Fevrier et al., 2005), A $\beta$ (Rajendran et al., 2006; Aguzzi and Rajendran, 2009), and tau (Saman et al., 2012; Wang et al., 2017b), sparking the interest in investigating the role of EVs in disease progression. As such, it is necessary to elucidate the underlying mechanisms which are responsible for inducing EV-mediated spread of these pathological molecules, how uptake of these EVs affect recipient cells, and how these outcomes differ in various CNS cell types.

Glial cells play significant roles in maintaining CNS homeostasis, regulating neuroinflammation, and supporting neurons, etc. Unsurprisingly, much of the focus of EV research in the realm of neuroscience has been centered on investigating the role of astrocyte (Bianco et al., 2009; Goetzl et al., 2016, 2018; Lafourcade et al., 2016; Luarte et al., 2017; Willis et al., 2017; Xin et al., 2017), microglial (Bianco et al., 2005; Potolicchio et al., 2005; Antonucci et al., 2012; Turola et al., 2012; Glebov et al., 2015), and oligodendrocyte (Krämer-Albers et al., 2007; Frühbeis et al., 2013) derived vesicles, and how these EVs may affect recipient neurons. Interestingly, like EVs derived from systemic immune cells, those secreted by microglia contain MHCII receptors and participate in the immune response (Potolicchio et al., 2005; Verderio et al., 2012). 


\subsection{Mitochondria}

The brain accounts for approximately $2 \%$ of the body's total weight, yet consumes $20 \%$ of the body's energy. Over half of this energy is required for powering and maintaining the sodium/potassium pump required for maintaining the membrane potential in neurons. Mitochondria are the powerhouses of the cell, and neurons critically depend on glucose metabolism and oxidative phosphorylation to meet these high energy demands, as they have no form of energy storage (Falkowska et al., 2015). Astrocytes have energy stores which can be tapped when glucose levels are low (Cataldo and Broadwell, 1986; Baltan, 2015); via the shuttling of lactate from astrocytes to neurons, which may be mediated by astrocyte-derived EVs containing monocarboxylate transporter 1 (Potolicchio et al., 2005), neurons can convert the donated lactate to pyruvate to be utilized in oxidative phosphorylation (Riske et al., 2017). Disruptions in neural energy metabolism can reduce ATP production, which even transiently, can have profound effects on neuronal viability (Simpkins et al., 2010).

Not only are mitochondria responsible for supplying the main source of energy for most cells, adenosine triphosphate (ATP), these organelles also play important roles in the cell, including signaling through reactive oxygen species (ROS) and the induction of apoptosis. Mitochondria are the main source of ROS (Balaban et al., 2005). Superoxide and hydrogen peroxide $\left(\mathrm{H}_{2} \mathrm{O}_{2}\right)$ are typical byproducts of mitochondrial oxidative phosphorylation as electrons are shuttled down the electron transport chain (ETC). Environmental factors, including TNF- $\alpha$ (Shoji et al., 1995;

Corda et al., 2001) and hypoxia (Guzy et al., 2005) can increase mitochondrial ROS production. These reactive molecules can induce oxidative stress by damaging DNA, lipids, and proteins, which can lead to cellular dysfunction and/or death (Gupta et al., 2012). High levels of ROS and can also alter NF- $\mathrm{BB}$ signaling, thereby modulating the immune response (Toledano et al., 1993; Kabe et al., 2005). Mitochondria can participate in the immune response through several other avenues. For example, various cytosolic PRRs can bind with mitochondrial antiviral signaling proteins (MAVS) expressed on the outer mitochondrial membrane, which can lead to the induction of NFאB activation (Meyer et al., 2018). Damaged or stressed mitochondria can leak mitochondrial DNA (mtDNA) into the cytoplasm of the cell and be sensed as a DAMP, which 
can also induce NF- $\mathrm{KB}$ activation (Collins et al., 2004). Improperly functioning mitochondria can be extremely detrimental to cells by producing oxidative stress, or by inducing sterile inflammation.

Apoptosis is also regulated by mitochondria via downstream activation of caspases. The B-cell lymphoma 2 (Bcl-2) protein family consist of both pro- and anti-apoptotic proteins. The antiapoptotic proteins (BCL-2, BCL-w, BCL-xL, A1, and MCL) are localized to the outer mitochondrial membrane (OMM), and work to inhibit the functions of the pro-apoptotic Bcl-2 proteins (including the effector proteins, BAK and BAX, and the essential apoptotic initiator, BH3-only proteins, BAD, BID, BIK, BIM, BMF, bNIP3, HRK, Noxa, and PUMA) (Chipuk and Green, 2008). The anti-apoptotic Bcl-2 proteins form heterodimers with BAK and BAX, sequestering them and preventing activation of pro-apoptotic pathways. Apoptotic stimuli induces BAK and BAX to form oligomers that insert themselves into the OMM, creating pores (Chipuk and Green, 2008). These channels allow for the efflux of cytochrome $c$ (cyt $c$ ) into the cytosol. Under normal conditions, cyt $c$ is anchored to the inner mitochondrial membrane via its interaction with cardiolipin. It is thought that there are two pools of cyt $c$, either tightly and loosely bound (Ott et al., 2002). Pore formation via BAK/BAX membrane insertion can trigger the release of loosely bound cyt $c$, while the tightly bound pool requires cardiolipin oxidation for dissociation and subsequent translocation to the cytosol (Ott et al., 2002; Orrenius and Zhivotovsky, 2005). Upon occurrence of this event, cyt $c$ forms an apoptosome complex with procaspase-9 and apoptotic protease-activating factor 1 (Apaf-1) (Sas et al., 2007). The apoptosome activates capsape-9, which subsequently activates procaspase-3 and/or procaspase-7, leading to caspase-3/7 induced apoptosis (Sas et al., 2007).

The mitochondrial genome (mtDNA) sequestered within the mitochondrial matrix, encodes for 13 proteins of the ETC; the remaining 1500 mitochondrial proteins are nuclear encoded (Taanman, 1999). Despite this, many of the genes encoded by mtDNA can be targeted by miRNAs. For instance, miR-34a, has been shown to target NDUFC2 of complex I; SDHC of complex II; UQCRB and QUCRQ of complex III, and COX10 of complex IV (Sarkar et al., 2016). MiR-34a-mediated translational repression can prevent replacement of these proteins after 
normal turnover. If this were to occur, the ETC would not be able to function properly, mitochondrial dysfunction would arise, and less ATP would be produced. Coincidently, we have identified an NF- $\mathrm{KB}$ binding site on the promotor region of miR-34a (Sarkar et al., 2016), suggesting that inflammatory stimuli which induce NF- $\kappa$ B activation also likely lead to the upregulated transcription of miR-34a. In turn, this could perpetuate inflammation as dysfunctional mitochondria contribute to increased ROS production and potential leakage of mtDNA into the cytosol. Reduced mitochondrial function has also been implicated in many neurodegenerative, neuroinflammatory diseases.

\subsection{Alzheimer's Disease}

Alzheimer's disease (AD) is a progressive, debilitating neurodegenerative disease which affects nearly 6 million Americans, and is the $6^{\text {th }}$ leading cause of death in the United States (Alzheimer's Association, 2016). The two hallmark lesions of the disease are intracellular neurofibrillary tangles of hyperphosphorylated tau, and extracellular aggregation of amyloid beta $(\mathrm{A} \beta)$ in the form of plaques, which are usually surrounded by dystrophic neurites and microglia. The disease is also characterized by wide-spread cellular death, enlarged ventricles, and significant reductions in cellular energy metabolism. At early stages of the disease, these pathologies are first detected in the entorhinal cortex; as time and the disease progress, pathology spreads into the temporal and frontal cortices, with much of the brain being affected at the later stages (Braak and Braak, 1991a).

Axonal cytoskeletal proteins are stabilized by the microtubule-associated phosphoprotein, tau (Binder et al., 1985; Buée et al., 2000; Kadavath et al., 2015). In AD and other tauopathies, tau becomes hyperphosphorylated and dissociates from microtubules; the microtubules are no longer stabilized and begin to break down, while the hyperphosphorylated tau begins to aggregate and form intracellular tangles. Due to the breakdown of the microtubules, axonal transport of key neurotrophic molecules necessary for neural signaling and survival is prevented, and cellular death ensues. These dying cells release factors known as damage associated molecular patterns (DAMPs), which can activate microglia for tissue clearance, and subsequently induce an immune 
response (Thundyil and Lim, 2015).

miRNAs have been shown to influence the expression and aggregation of tau. Both clinical evidence and transgenic animal models indicate that anxiety is a common psychiatric symptom experienced in AD (Teri et al., 1999; Blázquez et al., 2014), and it is likely due to tau dysregulation (Pristerà et al., 2013). Anxiety may be the result of upregulated miR-92a in AD brains; one target of this miR is vGAT, which is responsible for loading GABA, the main inhibitory neurotransmitter, into secretory vesicles (Li et al., 2017). Dysregulation of GABA signaling has been thought to contribute to anxiety, as neurons are left in an unregulated, overexcited state. Both the overexpression of vGAT and the administration of a miR-92a antagomir reduced anxiety-related behaviors in a mouse model of overexpressed human tau $(\mathrm{Li}$ et al., 2017), suggesting a crucial role for miR-92a in the GABA secretory pathway, with subsequent effects on anxiety behavior.

In the normal brain, amyloid precursor protein (APP) is constantly being cleaved (Selkoe, 1994; Akiyama et al., 2000b), producing both soluble APP $\alpha$ (sAPP $\alpha$ ) and sAPP $\beta$ (Nicolas and Hassan, 2014). Soluble APP $\alpha$ is non-pathogenic and does not accumulate, whereas sAPP $\beta$ can be processed to $A \beta 1-42$ (A $\beta 42)$, and aggregate into fibrils and plaques, characteristic of $A D$ (Nicolas and Hassan, 2014). As extracellular A $\beta 42$ accumulates and begins to form plaques, it attracts and activates microglia (Falcão et al., 2017), which leads to the observation of a barrierlike ring of microglia around the plaque (Eikelenboom et al., 2006; Uslu et al., 2012; Hong et al., 2016). $A \beta$ resides in the center of the plaque, and other molecules, such as complement factors, have also been observed within plaques (Eikelenboom et al., 1989). Complement-containing plaques are capable of inducing full blown complement cascades within the AD brain (Eikelenboom et al., 1989; Rogers et al., 1992; Fu et al., 2012), further contributing to chronic local inflammation. Additionally, intracellular accumulations of A $\beta 42$ have been observed within microglia (Akiyama et al., 1996), as A $\beta 42$ molecules can be phagocytosed by microglia prior to aggregation in a dose- and time-dependent manner (Kopec and Carroll, 1998). In healthy brains, this process occurs constantly (Ard et al., 1996), however in disease states, aggregated A $\beta$ can be 
engulfed, but not easily broken down, as it can remain un-degraded within the microglia for several days (Frackowiak et al., 1992).

$\mathrm{AD}$ is usually preceded by mild cognitive impairment. This condition is can be considered a precursor to dementia, and is characterized by noticeable, but non-severe changes in memory. It has been widely shown that adults diagnosed with $\mathrm{MCI}$ experience chronic brain vasculature hypoperfusion, which can lead to increases in $\mathrm{A} \beta$ deposits and death receptor-6 (DR6) protein, activation of caspases-3 and -6 , and dendrite degeneration (Chen et al., 2017b). The presence of DR6 has been implicated with neuronal loss and dendritic degeneration. miR-195 targets DR6 mRNA; upregulation of this miRNA has been demonstrated to reduce translation of DR6 protein, and subsequently diminish the loss of both dendrites and neurons (Chen et al., 2017b). ADlinked neuronal loss and impaired neurite differentiation has also been linked to upregulation of miR-211-5p; and these effects were found to be exacerbated in the presence of extracellular $A \beta$ (Fan et al., 2016). Longitudinal functional imaging studies also indicate that brain energy metabolism is impaired in these patients, and longitudinal studies indicate that these reductions in brain metabolism precede clinical onset of more severe symptoms characteristic of $\mathrm{AD}$ (Fouquet et al., 2009; Mosconi et al., 2009; Sperling et al., 2010). As such, mitochondrial dysfunction has been implicated in AD for decades (Castellani et al., 2002; Swerdlow and Khan, 2004). The mitochondrial cascade hypothesis posits that basal rates of mitochondrial function and ROS production are determined by inherited functionality of the ETC. With time, some individuals have a higher predisposition to greater accumulation of ROS-induced mtDNA damage, which creates a positive feedback loop by increasing ROS generation even further by increasing A $\beta$ formation (Swerdlow and Khan, 2004). Indeed, from a molecular standpoint, mitochondrial dysfunction leads to increases in APP processing towards the amyloidogenic pathway (i.e. producing A $\beta 42$ ) (Gabuzda et al., 1994; Atamna and Frey, 2007; Swerdlow et al., 2014). Our laboratory has recently demonstrated that miR-34a is significantly upregulated within the temporal cortex of post-mortem AD brains, as well as the temporal cortices and hippocampi of a triple transgenic mouse model of AD (3xTgAD) (Sarkar et al., 2016). Additionally, overexpression of miR-34a in primary neurons leads to subsequent mitochondrial dysfunction, as indicated by reduced mitochondrial respiration and ATP production (Sarkar et al., 2016). As 
such, abhorrent upregulation of miR-34a may be a main contributor dysregulated energy metabolism in the AD brain.

Unsurprisingly, many believe that in $\mathrm{AD}$, neuroinflammation is a driving force behind neurodegeneration (Akiyama et al., 2000b; Wyss-Coray, 2006). It is important to stress that inflammation, in it of itself, is not a negative phenomenon. In addition to fighting off infections, inflammatory responses are generated to remove and restore damaged tissue after injury. For example, intrahippocampal injection of lipopolysaccharide (LPS) in amyloid precursor protein (APP) transgenic mice triggers an inflammatory response, resulting in transient clearance of diffuse amyloid- $\beta$ (A $\beta$ ) deposits (Herber et al., 2004, 2007). Unfortunately, inflammation also has the capacity to damage healthy surrounding tissue as well (Laskin and Pendino, 1995). As such, chronic inflammation induced by the accumulation of extracellular A $\beta$ plaques, DAMPs released from dystrophic, necrotic cells, and accumulating mitochondrial ROS all appear to play pertinent roles in the pathophysiology of AD. None of these factors alone have proven adequate to explain the etiology of AD. The cause of this disease, and the driving force behind its progression, appears to be very complex and may rely heavily on genetic risk factors and regulatory mechanisms, which may easily implicate miRs in disease onset and progression.

\subsection{Stroke}

In recent years, the mortality rate of stroke has declined overall; however, it is still the 5th leading cause of death and one of the most prominent causes of disability in the United States (Yang et al., 2017). Every 40 seconds, someone in the United States experiences a stroke, and a stroke-associated death occurs about every four minutes (Mozaffarian et al., 2016). While the etiology of stroke is not fully understood, unhealthy lifestyle habits including poor diet, lack of exercise, hypertension, and smoking all appear to contribute heavily to increased stroke risk (Boehme et al., 2017). 
Strokes occur when a cerebral artery is blocked (ischemic stroke; $87 \%$ of cases) or ruptures (intracerebral or subarachnoid hemorrhage; 10 and 3\% of cases, respectively), resulting in a loss of blood flow to the brain area supplied by that artery (Benjamin et al., 2017). Although proven effective if administered to an eligible patient population, the therapeutic window of recombinant tissue plasminogen activator (tPA), the most widely adopted thrombolytic agent, is exceptionally short (3-4.5 hours from symptom onset), due to the increased risk of cerebral hemorrhage when given after that time point.

During a stroke, brain tissue closest to the ischemic event is deprived of oxygen, glucose, and other key nutrients and begins to die; this region is known as the ischemic core. The surrounding tissue remains at-risk for death, and is commonly referred to as the penumbra. Necrotic cells in the ischemic core release DAMPs which can activate microglia in the surrounding, penumbral region. The activation of these cells can lead to further production of inflammatory mediators, some of which can prompt further neuronal cell death, such as TNF- $\alpha$ and IL-1 $\beta$ (Zhang et al., 2012). Post-stroke apoptosis occurs through activation of the intrinsic or extrinsic apoptosis activation pathways. The intrinsic pathway activates mitochondrial mediated apoptosis, while the extrinsic pathway initiates signaling of death receptor pathways (which may also activate mitochondrial mediated apoptosis); both pathways result in the activation of caspase-3 and subsequent initiation of apoptosis (Broughton et al., 2009). By reducing mitochondrial-mediated apoptosis, Bcl-2 overexpression significantly reduces infarct volume in experimental stroke models (Cao et al., 2002).

Microglial activation, and subsequent cytokine release, has been shown to be inhibited by knockdown of miR-377 (Fan et al., 2017). In a rat MCAO stroke model, knocking down miR377 reduced ischemic infarct size by reducing inflammation mediated by microglial activation, and promoting angiogenesis, thus leading to better stroke outcomes (Fan et al., 2017). As inflammation-induced post-stroke upregulation of TNF- $\alpha$ occurs, there is a pronounced downregulation of miR-181c in activated rat microglial cells; this relationship can be reversed by transfecting microglia with miR-181c, thus decreasing levels of TNF- $\alpha$, reducing inflammation, and subsequently reducing neuronal cell death (Zhang et al., 2012). Indeed, genetically knocking 
out signaling components of the TNF- $\alpha$ death receptor pathway significantly reduced infarct volume in experimental stroke models (Martin-Villalba et al., 1999).

Favorable stroke outcomes rely partially on the integrity of the BBB post-insult. As previously discussed, the BBB can be compromised during periods of inflammation, and inflammation induced via acute ischemic stroke is no different. Recent data implicate significant post-stroke decreases of miR-122 in whole blood of both stroke patients and animal models (Liu et al., 2016a). Targets of miR-122 include proteins that affect cell adhesion and leukocyte extravasation. When miR-122 was administered into the blood, lower infarct volume and better neurological outcomes were observed as compared to when it was directly injected into brain ventricles, indicating that this miRNA is likely affecting CNS leukocyte invasion via peripheral mechanisms rather than directly influencing CNS tissue (Liu et al., 2016a). In the stroke-prone spontaneously hypertensive rat model, it was shown that prior to onset of stroke, levels of miR122 within the brain are decreased substantially. Additionally, vascular endothelial cells showed heightened levels of inflammation and death, leading to degradation of the BBB, however these responses were attenuated after exposure to miR-122 (Stanzione et al., 2017). Together, these data suggest that miR-122 acts through the blood to improve the integrity of endothelial cells comprising the BBB.

In the same vein, miR-126 and miR-126* also appear to regulate leukocyte adhesion and infiltration across the BBB (Cerutti et al., 2017). These miRs are downregulated in response to pro-inflammatory cytokines, such as TNF- $\alpha$, which is upregulated post-stroke. When these miRNAs are downregulated, VCAM1 and E-selectin levels are increased, respectively, and leukocyte adhesion significantly increases (Cerutti et al., 2017), which can lead to enhanced leukocyte extravasation into the brain, thus worsening patient outcomes. Additionally, miR-126 has been shown to facilitate vascular remodeling after cardiovascular insult, such as stroke. In a mouse line with conditional endothelial cell knock-out of miR-126, knockout mice fared worse than controls post-stroke, exhibiting higher levels of inflammation and cardiac hypertrophy (Chen et al., 2017a). Stroke-induced miR-126 downregulation appears to play a large role in outcomes related to inflammation and overall cardiovascular health after insult. 
The relationship between stroke and infection has also gained attention over the past several decades, and recent infection is now considered a stroke risk factor (Grau et al., 1995, 2005; Bova et al., 1996; Paganini-Hill et al., 2003). Systemic immune activation can significantly contribute to thrombosis formation (Maurer et al., 2010), and thus increase the risk of experiencing an ischemic stroke. Post-stroke infection is also a major cause of concern. Immunosuppression is a common ailment seen in stroke patients (Cruse et al., 1992; Członkowska et al., 1979; Meisel et al., 2005), and can commonly lead to development of serious infections including pneumonia and urinary tract infections (Johnston et al., 1998; Weimar et al., 2002). Lymphocyte counts are significantly reduced post-stroke, and those that remain, exhibit a reduced ability to respond to inflammatory stimuli indicating that there is reduced efficacy of the innate immune system signaling (Prass et al., 2003). As both stroke and inflammation contribute to altering transcription of miRNAs, it is highly likely that these factors work in a concerted fashion to mediate stroke onset and outcome severity.

\subsection{Gaps in Knowledge}

The above literature review introduces a broad range of topics, with an overarching focus on neuroinflammation, and its associated diseases.

\section{miRNAs}

Pro-inflammatory stimuli like LPS and TNF- $\alpha$ are clearly implicated in the onset and progression of neuroinflammation, however their effects on miRNA expression are a newer area of research. Understanding the genetic nexus of neuroinflammation would provide new insights on underlying disease pathology, mechanisms of progression, and more importantly, pathways and targets for therapeutic intervention. As of 2015, over 2000 miRNAs had been identified (Hammond, 2015), and it's likely that this number is even larger now. As such, it is impossible to study such a vast quantity of molecules with highly complex, network-level signaling effects. 
Our approach, as with many other groups, is to find a subset of miRNAs of interest, and explore their role in various disease states. In this regard, we focus on the miRNAs, miR-34a and -146a. We have previously demonstrated that in the AD brain, they are significantly upregulated in a disease-stage specific manner. Understanding how this relates to the pathophysiology of disease progression will shed light on how inflammatory stimuli influence their expression, as well as the functional consequences of this. AD progression is characterized by chronic neuroinflammation and reduced brain energy metabolism, and potential targets of miR-34a and 146a include several proteins involved in cellular bioenergetics. Understanding how these miRNAs fit into the broader picture of inflammation and mitochondrial dysfunction could provide insight on the potential benefits of utilizing these miRNAs as therapeutic targets in disease.

\section{Neuronal EVs}

As inflammation has been shown to affect the biogenesis and secretion of EVs as well, it comes as no surprise that the study of EVs in the context of neuroinflammation is also gaining traction. Much of the work in this field however, focuses more so on EVs derived from glial cells. Functional studies regarding neuronal uptake of EVs is also within the context of glial cellderived EVs. Many groups focusing on neuronal-derived EVs are interested in the clinical and diagnostic aspects of these vesicles, via the identification and development of assays for identifying neuronal-derived EVs in circulating biological fluids (e.g. plasma, CSF) (Saman et al., 2012; Mustapic et al., 2017; Sun et al., 2017). Many other studies focusing on neuronal derived EVs concentrate on the effects of synaptic activity on EV production, and their role in cell-to-cell communication (Wang et al., 2017b; Xu et al., 2017; Polanco et al., 2018). There are, however, some groups whom do focus on the effects inflammation or injury-induced neuronal EVs (Putz et al., 2008; Simeoli et al., 2017). To our knowledge, no other groups have assessed the bioenergetic outcomes of neuronal uptake of inflammation-induced neuronal-derived EVs.

\section{Intermittent LPS Exposure}


Interactions between the systemic immune and central nervous systems are highly complex. Stroke risk factors are associated with unhealthy lifestyle habits, which may increase systemic inflammation. Others have demonstrated that LPS exposure, when administered between 1 and 7 days of experimental stroke, is neuroprotective and leads to smaller infarct volumes (Rosenzweig et al., 2004, 2007; Stevens et al., 2008). In line with this proposed timeframe, we previously demonstrated that exposure to lipopolysaccharide (LPS) 30 minutes prior to experimental stroke significantly increases stroke infarct volume (Doll et al., 2015b). Evidence examining repeated LPS exposure appears limited to repeated dosing over several consecutive days prior to stroke (Rosenzweig et al., 2007), however this paradigm is not clinically relevant. To model more closely the rate of sickness experienced in humans, we were interested in determining the effects of intermittent LPS exposure, from which animals recover, and the impact this may have on stroke outcomes. 


\section{Chapter 2}

\section{miR-146a dysregulates energy metabolism during neuroinflammation}

\section{Sujung Jun*, Ashley E. Russell*, Wei Wang, Darren E. Gemoets, Saumyendra N. Sarkar, James W. Simpkins, and Candice M. Brown}

*Co-first authors

Author Contribution: SJ, SS, JWS and CMB designed studies; SJ, AR, WW, SS performed research; SJ, AR and CMB analyzed data; SJ, AR, JWS and CMB wrote the paper.

Jun S*, Russell AE*, Wang W, Gemoets DE, Sarkar S, Simpkins JW, Brown CM (2019) miR-146a dysregulates energy 
metabolism during neuroinflammation. Neuroscience. In revision. 


\subsection{Abstract}

Alzheimer's disease (AD) and other neurodegenerative diseases are characterized by chronic neuroinflammation and a reduction in brain energy metabolism. An important role has emerged for small, non-coding RNA molecules known as microRNAs (miRNAs) in the pathophysiology of many neurodegenerative disorders. As epigenetic regulators, miRNAs possess the capacity to regulate and fine tune protein production by inhibiting translation. Several miRNAs, which include miR-146a, are elevated in the brain, CSF, and plasma of AD patients. miR-146a participates in pathways that regulate immune activation and has several mRNA targets which encode for proteins involved in cellular energy metabolism. An additional role for extracellular vesicles (EVs) has also emerged in the progression $\mathrm{AD}$, as EVs can transfer functionally active proteins and RNAs from diseased to healthy cells. In the current study, we exposed various cell types present within the CNS to immunomodulatory molecules and observed significant upregulation of miR-146a expression, both within cells and within their secreted EVs. Further, we assessed the effects of miR-146a overexpression on bioenergetic function in primary rat glial cells and found significant reductions in oxidative phosphorylation and glycolysis. Lastly, we correlated miR-146a expression levels within various regions of the AD brain to disease staging and found significant, positive correlations. These novel results demonstrate that the modulation of miR-146a in response to neuroinflammatory stimuli may mediate the loss of mitochondrial integrity and function in cells, thereby contributing to the progression of beta-amyloid and tau pathology in the $\mathrm{AD}$ brain. 


\subsection{Introduction}

Many neurodegenerative diseases, including Alzheimer's disease (AD), are characterized by chronic neuroinflammation. Prolonged, unregulated inflammation leads to the increased production of various pro-inflammatory molecules, which likely contribute to disease progression. Although the genetics of late-onset Alzheimer's disease are complex, recent studies have observed significant downregulation of many genes necessary for proper cellular bioenergetics (Liang et al., 2008). Moreover, functional imaging studies of patients suffering from $\mathrm{AD}$ reveal that the severity of brain hypometabolism is correlated with dementia (Brier et al., 2012; Yin et al., 2016). It is clear that dysfunctional brain energetics is an important aspect of AD pathology, yet the molecular mechanisms responsible are poorly understood.

Due to their ability to regulate translational repression of multiple mRNA targets, microRNAs (miRNAs) have recently been implicated as major players in the pathogenesis of AD. A deleterious, pro-inflammatory role has emerged for miR-146a in AD (Alexandrov et al., 2014), as well as other neurological (Nguyen et al., 2018) and degenerative diseases (Pogue and Lukiw, 2018). Classically, miR-146a has been considered a well-characterized anti-inflammatory miRNA, as several mRNA targets include genes that encode for interleukin 1 receptor associated kinase (IRAK1), TNFR-associated factor 6 (TRAF6), and signal transducer and activator of transcription 1 (STAT1) (Taganov et al., 2006; Cui et al., 2010; Iyer et al., 2012; Wang et al., 2013). Many of these proteins work as transcription factors to increase production of inflammatory cytokines or induce apoptosis; therefore miR-146a mediated translationalrepression of these signaling molecules can reduce inflammatory responses within cells. In contrast, more recent studies also suggest a pro-inflammatory role for miR-146a function and have observed increased expression of miR-146a in response to oxidative stress and inflammation (Lukiw and Alexandrov, 2012; Wu et al., 2015; Cardoso et al., 2016). Elevated levels of miR-146a have also been shown to downregulate expression of complement factor $\mathrm{H}$ (CFH), an important repressor of inflammation (Lukiw et al., 2008). Thus, a growing body of literature strongly suggests that miR-146a participates in both pro- and anti-inflammatory roles in human health and disease. 
In addition to the physical hallmarks of $\mathrm{AD}$ such as extracellular $\mathrm{A} \beta$ plaques, intracellular neurofibrillary tangles, and neuronal loss, widespread hypometabolism is also observed within the AD brain (de Leon et al., 2001; Mosconi et al., 2006, 2008). Interestingly, several mRNAs encoding for proteins involved in cellular metabolism have recently been identified as targets of miR-146a; these proteins have been demonstrated to play important roles in glycolysis, mitochondrial respiration, mitochondrial transport, and apoptosis (Rippo et al., 2014). Due to its inflammation-induced upregulation, and the mRNAs in which it targets, miR-146a may be a key player in the pathophysiology and progression of AD.

How miR-146a-mediated inflammatory signals accelerate or exacerbate AD progression is unclear. It is well-established that $\mathrm{AD}$ typically progresses in a very characteristic manner with initial pathology developing in the entorhinal cortex, then spreading throughout the temporal cortex, and finally into the frontal cortex (Smith, 2002). Propagation of disease pathology from one cell to another may be due, in part, to communication between cells via extracellular vesicles (EVs). EVs are small, nano-sized particles shed or excreted from most cell types, which have the capacity to transfer functional RNAs, including miRNAs, from one cell to another (Valadi et al., 2007; van Niel et al., 2018). Thus, EVs have been implicated in the spread of pathological molecules during disease states that include $\mathrm{AD}$ and other neurodegenerative diseases (reviewed by Thompson et al., 2016).

Previous work from our laboratory and others, have shown that miR-146a is significantly upregulated in the brains of $\mathrm{AD}$ patients, compared to their age-matched controls (AMCs) (Sarkar et al., 2016; Pogue and Lukiw, 2018). In the current study, we assessed the effects of various inflammatory mediators on different cell types within the CNS, the functional bioenergetic outcomes of miR-146a overexpression, and the relationship between human brain miR-146a expression and AD pathology. We hypothesized that miR-146a expression would increase after exposure to inflammatory stimuli, and that overexpression of this miRNA would lead to decreased cellular bioenergetics. Further, we hypothesized that AD brains would have significantly higher levels of miR-146a expression when compared to age matched controls 
(AMCs), and these levels would positively correlate with disease severity, and negatively correlate with total mitochondrial protein expression.

\subsection{Experimental Procedures}

\section{Animals}

Pregnant rats (Sprague Dawley) were acquired from Hilltop Laboratory (Scottdale, PA) to obtain E18 brain tissue for preparation of mixed glial and cortical neuronal cultures. Three timedpregnant female rats were used for each primary culture experiment, with 6-10 embryos per dam, and each experiment was repeated three times. Therefore, nine timed pregnant female rats were used for all animal experiments. All animal studies were approved by the Institutional Animal Care and Use Committee at West Virginia University.

\section{Cell culture}

Primary culture: Pregnant rats were euthanized and fetal brain tissue was harvested from E18 rats. Brains were removed and placed into magnesium $\left(\mathrm{Mg}^{2+}\right)$ free Hank's balanced salt solution (HBSS). Cortices and hippocampi were removed under a dissecting microscope, washed, and placed into neurobasal culture media (without phenol red) supplemented with 1X B27 and 1\% penicillin-streptomycin (Gibco, Carlsbad, CA). The cortices and hippocampi were triturated using a graded series of fine polished Pasteur pipettes, and then filtered through a $40 \mu \mathrm{m}$ nylon cell strainer (Becton Dickinson Labware, Franklin Lakes, NJ). Neurons were plated on poly-Llysine coated $150-\mathrm{mm}$ dishes and cultured in vitro in $95 \%$ humidity and $5 \% \mathrm{CO}_{2}$ atmosphere at $37^{\circ} \mathrm{C}$ for 15 days. At day 2, cells were exposed to $5 \mu \mathrm{M} 1-\beta-\mathrm{D}$ - arabinofuranosyl cytosine (AraC) to inhibit glial cell growth as previously described (Sarkar et al., 2015, 2016). To obtain glial cells, following trituration the remaining cortical and hippocampal cell suspension was grown in DMEM for 4-5 days until a mixed glial population of astrocytes and microglia reached confluency.

Immortalized cell lines: A hippocampal neuronal cell line, HT-22 cells, was obtained from Dr. David Schubert at the Salk institute. Additionally, a cerebellar microglial cell line, C8-B4 cells, 
and a brain microvascular endothelial cell line, bEnd.3 cells, were both obtained from ATCC (Manassas, VA). All cell lines were of murine origin and grown in Dulbecco's Modified Essential Medium (high glucose) (Hyclone) with 10\% FBS 1\% pen/strep in humidified 95\% air $/ 5 \% \mathrm{CO} 2$ at $37^{\circ} \mathrm{C}$.

\section{Human brain tissues}

Brain tissue from the temporal cortex, frontal cortex, and cerebellum of confirmed AD patients and AMCs was obtained from the Kathleen Price Bryan Brain Bank, Bryan Alzheimer's Disease Research Center, Duke University Medical Center, Durham, NC. All tissues were staged for AD pathology with Braak and Braak staging (Braak and Braak, 1991b) and cerebral amyloid angiopathy (CAA) (Thal et al., 2003). Brain tissues were used in accordance with the institutional review board/ethical guidelines of the donor institution.

\section{Preparation of soluble amyloid-beta $(A \beta)$}

Synthetic A $\beta 1-42$ (Tocris, Ellisville, MO) oligomers were prepared without the fibrillar component according to published methods (Barghorn et al., 2005; Sarkar et al., 2015). Briefly, A $\beta 1-42$ was dissolved in 1,1,1,3,3,3- hexafluoro-2-propanol to $1 \mathrm{mM}$. The clear solution was then evaporated to dryness. Dried peptide was diluted in DMSO to $5 \mathrm{mM}$ and sonicated for 10 min in a bath sonicator. The peptide solution was resuspended in cold Neurobasal medium and immediately vortexed. The solution was then incubated at $4^{\circ} \mathrm{C}$ for $24 \mathrm{~h}$. After high speed centrifugation, the supernatant was collected, visualized by polyacrylamide gel electrophoresis and silver staining, and found to be comprised of fibrillar-free oligomers and monomers.

\section{Extracellular vesicle isolation}

HT-22 cells were seeded in $100 \mathrm{~mm}$ dishes and exposed to $1 \mathrm{ng} / \mathrm{ml}$ tumor necrosis factor-alpha (TNF- $\alpha$ ) when cells reached 80\% confluency. C8-B4 and bEnd.3 cells were seeded in $150 \mathrm{~mm}$ dishes, and when cells reached 50\% confluency, they were exposed to either $1 \mu \mathrm{g} / \mathrm{ml} \mathrm{LPS}$ (Sigma-Aldrich, St. Louis, MO) or $1 \mathrm{ng} / \mathrm{ml}$ TNF- $\alpha$ (R\&D, Minneapolis, MN). Similarly, rat cortical primary neuronal and glial cells were grown in $150 \mathrm{~mm}$ dishes and when cells were $75 \%$ confluent, they were exposed to 250 or $500 \mathrm{nM}$ of oligomeric A $\beta$. For all cell types and exposure 
conditions, after 24 hours, the media was collected for EV isolation and downstream miRNA purification. EVs were isolated from the cell culture media by either ultracentrifugation (Théry et al., 2006; Sarkar et al., 2015) or ExoRNeasy Isolation Kit (Qiagen, Germantown, MD) according to the manufacturer's protocol. Using the ultracentrifugation method, the conditioned cell culture media was centrifuged ( $320 \mathrm{x}$ g for 5 minutes) to remove cells, and the supernatant was filtered through a Steriflip filter $(0.22 \mu \mathrm{m}$ pore size $)$. EVs were pelleted from the filtered media by ultracentrifugation at $100,000 \mathrm{~g}$ for $60 \mathrm{~min}$ at $4^{\circ} \mathrm{C}$ using Beckman SW 28 rotor running in a Beckman Coulter Optima L-100 XP ultracentrifuge (Beckman Coulter, Brea, CA). EVs were washed once with sterile PBS, collected by ultracentrifugation, and resuspended in $1 \mathrm{ml}$ of sterile PBS.

\section{Overexpression of miR-146a in primary mixed glial cells}

To induce overexpression of miR-146a in cells and their secreted EVs, rat primary mixed glial cells were transfected with either vector or miR-146a expression plasmid DNA by Lipofectamine 2000 (Invitrogen, Carlsbad, CA). The miR-146a expression vector was purchased through Addgene (Addgene plasmid \#15092, Cambridge, MA), which was deposited from David Baltimore (Taganov et al., 2006). Forty hours after transfection, EVs in the conditioned media were prepared as described above. The presence or absence of miR-146a was determined by isolating total RNA using the miRNeasy Kit (Qiagen) followed by qRT-PCR as described below.

miRNA expression analysis

Total RNA enriched in miRNAs was isolated from approximately $5 \mathrm{mg}$ of individual frozen brain tissue samples and various cell samples by using the miRNeasy Micro Kit Isolation Kit (Qiagen). Total RNA enriched in miRNAs was also isolated from EVs using the miRNeasy Serum/Plasma Kit. Prior to isolation of EV RNA, $3.5 \mu 1$ C. elegans miR-39 (at a concentration of $1.6 \times 10^{8}$ copies/ $\mu$ l) was added to each sample as a spike-in control. Total RNA concentrations were measured using a Nanodrop 2000 spectrophotometer (Thermo Scientific, Waltham, MA). Using the miScript II RT Kit (Qiagen), $2 \mu \mathrm{g}$ of total RNA containing miRNA was reverse transcribed in a total volume of $10 \mu \mathrm{l}$ reaction mix to make cDNA. Expression of miR-146a was determined by quantitative RT-PCR, which was performed using target specific miScript primer 
assays and the miScript SYBR® Green PCR Kit (Qiagen). All reactions were performed in triplicate for each sample using a StepOne Plus PCR system (Applied Biosystems, Foster City, CA) for 40 cycles as follows: $10 \mathrm{sec}$ at $95{ }^{\circ} \mathrm{C}, 30 \mathrm{sec}$ at $55^{\circ} \mathrm{C}, 30 \mathrm{sec}$ at $70{ }^{\circ} \mathrm{C}$. Negative control reactions were included as wells containing only master mix and nuclease-free water without any template cDNA. All miRNA specific primers were from Qiagen and included: miScript Primer Assay Hs_miR-146a (MS00003535), Hs_RNU6 (MS00033740), and Ce_miR-39_1 (219610). The expression levels of miR-146a were standardized against those of RNU6 (an internal control for brain tissue and cell samples) and miR-39 (a spike-in control for EV samples) detected in identical cDNA samples. Quantification of PCR amplified miRNA specific cDNA was done by the comparative cycle threshold CT method $(\Delta \Delta \mathrm{CT})$ (Livak and Schmittgen, 2001).

\section{Measurement of mitochondrial respiration: oxygen consumption rate (OCR)} Approximately 20,000 primary mixed glial cells per well were seeded in a XFe 96 cell culture microplate (Agilent, Santa Clara, CA) in DMEM medium with 10\% FBS and 1\% pen/strep. The next day, glial cells were transfected with either vector or miR-146a expression plasmid DNA (1, 2, 3, or $4 \mu \mathrm{g} / \mathrm{ml}$ ) by Lipofectamine 2000 (Invitrogen) according to the manufacturer's protocol. Forty hours after transfection, OCR was measured by the XFe 96 Extracellular Flux Analyzer using a Mito Stress Kit (Agilent) according to the manufacturer's protocol. To measure mitochondrial function in real time, oligomycin, carbonyl cyanide p-[trifluoromethoxy]-phenylhydrazone (FCCP), and antimycin A plus rotenone were added separately to a sensor cartridge and injected sequentially through ports in the XF Assay cartridge to final concentrations of 1 $\mu \mathrm{g} / \mathrm{ml}, 1 \mu \mathrm{M}$ and $10 \mu \mathrm{M}$, respectively. Addition of these reagents allowed for measurement and calculation of the basal level of mitochondrial oxygen consumption, the amount of oxygen consumption linked to ATP production, the level of non-ATP-linked oxygen consumption (proton leak), the maximal respiratory capacity, and the non-mitochondrial oxygen consumption. For each experimental condition six replicate wells were used.

\section{Measurement of glycolytic function: extracellular acidification rate (ECAR)}

Similarly, as performed for OCR, primary mixed glial cells were cultured in a XFe96 cell culture microplate and transfected with vector or miR-146a expression plasmid DNA. Forty hours after 
transfection, ECAR was measured by XFe 96 Extracellular Flux Analyzer using a Glycolysis Stress Kit (Agilent) which measures three key parameters of glycolytic function: glycolysis, glycolytic capacity, and glycolytic reserve. A saturating concentration of glucose, oligomycin, and 2-deoxy-glucose (2-DG; a glucose analog) were added separately in a sensor cartridge and injected sequentially through ports in the cartridge to final concentrations of $10 \mathrm{mM}, 0.6 \square \mathrm{M}$, $125 \mathrm{mM}$, respectively. This assay allows for the calculation of the basal rate of glycolysis/glycolytic flux, maximum glycolytic capacity, and glycolytic reserve. For each experimental condition, six replicate wells were used.

\section{Western blot analysis}

To prepare total protein extracts, frozen human brain tissues were homogenized in in Pierce® RIPA buffer (Thermo Scientific) with 100x Halt ${ }^{\mathrm{TM}}$ Protease Inhibitor Single-Use Cocktail (Thermo Scientific), followed by centrifugation at 13,400 $\mathrm{g}$ for $5 \mathrm{~min}$ at room temperature. The supernatant was collected and the protein concentrations were determined using Pierce ${ }^{\mathrm{TM}} \mathrm{BCA}$ Protein Assay (Thermo Scientific). Approximately $15 \mu \mathrm{g}$ total protein was separated by size in Bolt 4-12\% Bis-Tris Plus poly-acrylamide gels (Novex®, Thermo Scientific) through electrophoresis, along with the Chameleon ${ }^{\mathrm{TM}}$ Kit Pre-stained Protein Ladder (LI-COR, Lincoln, NE) size marker. Proteins were transferred to iBlot ${ }^{\circledR} 2$ PVDF membranes (Invitrogen, Thermo Scientific) by iBlot ${ }^{\circledR} 2$ Gel Transfer Device (Life Technologies). The membranes were blocked in Odyssey blocking buffer in TBS (LI-COR) for 1 hour at room temperature, and then incubated with primary antibodies of target proteins in sequential manner overnight at $4^{\circ} \mathrm{C}$ : VDAC (MilliporeSigma, Burlington, MA, (MABN504), 1:1,000 dilution), and $\beta$-actin (Adipogen (YIFLF-PA0209) 1:15,000 dilution). The following day, membranes were washed with 1x TBST buffer (prepared with 1x Tris buffered saline with 0.1\% Tween 20) 3 times for 5 minutes, and incubated with appropriate anti-rabbit, anti-mouse, secondary antibody conjugated with fluorescence dye (LI-COR, IRDye 800CW anti-Rabbit and anti-Mouse, both 1:15000 diluted in blocking buffer) for one hour, then washed again in 1x TBST 3 times in 5 minute intervals prior to imaging. The specific reaction was visualized by using the Odyssey CLx (LI-COR), and immunoblotting bands were quantified by densitometry using Image Studio 5.1. 


\section{Statistical analyses}

All experiments were repeated at least 3 times with $n=3-6$ wells/treatment/experiment. Results from the experiments are reported as means \pm SEM. Quantitative results that satisfied criteria for parametric analyses were assessed for significance using Welch's t-test for two sample comparisons or a one-way ANOVA with Tukey's post hoc test for three or more comparisons. Data in Figures 3 and 4 were analyzed using regression models where miRNA is the independent variable and ATP production, maximal respiration, protein leak, and spare capacity were dependent variables; all models showed a significant negative linear trend. Spearman's $\rho$ was calculated to determine correlations between miR-146a levels and Braak or CAA staging. Specific details of the statistical reporting for each experiment are provided in the corresponding figure legend. All analyses were performed using R 3.5.1 (https://wwww.R-project.org) and GraphPad Prism 8.0 (GraphPad Software, La Jolla, CA). A p value $\leq 0.05$ was used to establish significance; significance presented as $* p \leq 0.05, * * p \leq 0.01$, and $* * * p \leq 0.0001$.

\subsection{Results}

Neuroinflammatory stimuli induce upregulation of miR-146a in several CNS cell types and their EVs

Due to the heterogeneity of cell types within the CNS, our first goal was to understand how diverse inflammatory stimuli influence miR-146a production in various cells and their released EVs. To this end, we utilized an immortalized neuronal cell line (HT-22), a microglial cell line (C8-B4), and a brain endothelial cell line (bEnd.3). Cells were exposed to either TNF- $\alpha$ (1 $\mathrm{ng} / \mathrm{ml}$ ), a proinflammatory cytokine which is upregulated in AD (Decourt et al., 2016), or lipopolysaccharide (LPS) $(1 \mu \mathrm{g} / \mathrm{ml})$, which may be released from bacteria in the gastrointestinal tract and cross the aged BBB (Zhao and Lukiw, 2018), for 24 hours. TNF- $\alpha$ exposure of HT-22 cells significantly increased miR-146a expression both intracellularly (Fig 1A) and in secreted EVs (Fig 1D). Levels of miR-146a in C8-B4 cells were also significantly increased in response to LPS exposure both intracellularly (Fig 1B) and in secreted EVs (Fig 1E). A similar pattern of miR-146a expression was also examined in bEnd.3 cells (Fig 1C) and EVs (Fig 1F) exposed to LPS, however TNF- $\alpha$ exposure appears to affect these cells in a different manner. Although 
exposure to this cytokine induced significant upregulation of miR-146a in bEnd.3-derived EVs (Fig 1F), intracellular expression of miR-146a is significantly reduced. These data suggest the possibility of cell-type specific, as well as stimulus specific regulatory mechanisms, which influence miRNA packaging and secretion in EVs.

A $\beta$ exposure induces upregulation of miR-146a in primary neurons and mixed glial cells and their EVs

To determine if neuroinflammation-induced alterations in miR-146a were species specific, we next used rat primary neurons and primary mixed glial cells. For 24 hours, these cells were exposed to $\mathrm{A} \beta$; a neuroinflammatory component of plaques and one of the hallmarks of $\mathrm{AD}$. We observed a significant upregulation of miR-146a expression in primary neurons (Fig 2A), and a dose-dependent increase in their secreted EVs (Fig 2C). Exposure of primary mixed glia to A $\beta$ induced similar responses, with a dose-dependent increase of miR-146a expression intracellularly (Fig 2B), and a significant upregulation within their secreted EVs (Fig 2D).

miR-146a inhibits oxidative phosphorylation and glycolysis in primary mixed glial cells Previous work has shown that miR-146a targets several mRNAs which encode for proteins of the mitochondrial electron transport chain (Rippo et al., 2014) and glycolysis (Raschzok et al., 2011). Hence, we hypothesized that elevated levels of miR-146a would lead to significant impairment of cellular bioenergetics. To test this, we overexpressed miR-146a in rat primary mixed glial cells and measured oxidative phosphorylation and glycolysis using the Mito Stress Kit or Glycolysis Stress Test Kit (Agilent Biosciences). To ensure transfection efficiency prior to use of either of the metabolic stress kits, rat primary mixed glial cells were transfected with either vehicle vector or miR-146a overexpression vector, and cultured for 40 hours. We then assessed miR-146a expression levels intracellularly and in secreted EVs qRT-PCR. As shown in Table 1 when compared to vehicle vector, transfection of the miR-146a overexpression vector increased intracellular miR-146a levels more than 2-fold, while EV levels were increased in a dose-dependent manner (53 to 116-fold). In addition, we assessed cellular viability 40 hours after transfection with a calcein AM assay and observed no effects on cellular viability (data not shown). 
We next examined the effects of miR-146a overexpression on oxidative phosphorylation. After 40 hours of transfection, we observed a dose-dependent decrease in all OCR parameters assessed, including ATP production (Fig 3A), spare capacity (Fig 3B), maximal respiration (Fig 3C), and proton leak (Fig 3D) when compared to vector transfected cells. In addition, ECAR values were also measured 40 hours after transfection. Similarly, we observed a dose-dependent decrease in glycolysis (Fig 4A), glycolytic capacity (Fig 4B), and glycolytic reserve (Fig 4C) when compared to vector transfected cells. These data suggest that miR-146a has the capacity to alter cellular bioenergetics by impairing both mitochondrial function and glycolysis.

miR-146a expression is positively correlated with pathological scores in human AD brains Several groups have demonstrated that miR-146a levels are significantly increased in neocortical brain regions of AD patients, along with multiple biological fluids including serum, plasma, and cerebrospinal fluid (Alexandrov et al., 2012; Lukiw et al., 2012; Denk et al., 2015; Dong et al., 2015; Kumar and Reddy, 2016). To test if miR-146a expression exhibits a brain region specific phenotype, we analyzed miR-146a levels from the temporal cortex (TC), frontal cortex (FC), and cerebellum (CB) of 10 control and 13 AD patient brains. In accordance with previous reports by Lukiw et al. (2012), we confirmed that miR-146a levels are significantly increased in the TC of AD patients (3.6-fold) when compared to AMCs (Fig 5A). We also observed increased levels of miR-146a in the FC (5.3-fold; Fig 5D) and CB (3.7-fold; Fig 5G), but this trend did not reach statistical significance due to high variation among samples.

To test if the variation was due to the severity of AD pathology, we further analyzed miR-146a levels in brains when stratified by Braak stage (3 and 6). The increase of miR-146a in AD brains is most highly correlated with Braak stage 6, which designates late stage AD, regardless of which brain region we assessed; 5.7-fold in TC (Fig 5B), 8.6-fold in FC (Fig 5E), and 6.8-fold in CB (Fig 5H). We observed a significant positive correlation between increased levels of miR146a and the severity of Braak staging of AD patients for all three of the assessed brain regions (Figs 5C, 5F, and 5I). Further correlational analyses also indicated that levels of miR-146a were positively correlated with CAA. These data suggest that both tau pathology (as assessed with 
Braak staging) and amyloid burden on cerebral vasculature (as assessed with CAA) are correlated with increased levels of miR-146a.

Due to our finding that overexpression of miR-146a in primary mixed glial cells significantly reduces cellular bioenergetics, and miR-146a expression is significantly elevated in AD brains, we next probed for changes in total mitochondrial protein (VDAC1) levels within the temporal cortices of $\mathrm{AD}$ and $\mathrm{AMC}$ brains (Fig 6A). We found a significant negative correlation between increased expression levels of miR-146a and decreased mitochondrial protein (Fig 6B). Together, our findings suggest that as $\mathrm{AD}$ progresses, expression of miR-146a increases and is correlated with decreased expression of mitochondrial protein.

\subsection{Discussion}

In the current study, we have described a novel role for miR-146a in the pathophysiology of AD. We demonstrated that various inflammatory stimuli induce upregulation of miR-146a in several cell types of both mouse and rat origins. Our assessment of the functional impacts of elevated levels of miR-146a on cellular bioenergetics reveal novel and very interesting findings which indicate that further mechanistic probing is warranted. In addition to our in vitro studies, we utilized human brain samples to demonstrate that patients suffering from AD express significantly elevated levels of miR-146a when compared to AMCs. From these data, we propose that miR-146a plays a key role in the progression and pathophysiology of AD.

During periods of neuroinflammation, microglia and astrocytes act as the first responders and initiate an immune response (Dong and Benveniste, 2001; Yang et al., 2010). This response can be beneficial in the short term, as invading pathogens or aberrantly expressed molecules are cleared to protect and preserve neuronal function (Sochocka et al., 2017a). The negative consequences of neuroinflammation arise when the inflammatory response becomes prolonged, or chronic, such as the case for patients suffering from AD (Sochocka et al., 2017b). As AD progresses, the cells of the CNS are exposed to many inflammatory stimuli, including cytokines, chemokines, and $A \beta$ aggregates (Akiyama et al., 2000a). Here, we have shown that with 
prolonged exposure to these immunomodulatory molecules, miR-146a expression is significantly increased. In line with our results, other recent studies have also shown that miR-146a expression occurs within cells of the CNS, including choroid plexus epithelium (Balusu et al., 2016), human neural cells (Lukiw et al., 2008), and brain endothelial cells (Wu et al., 2015). Similar to our findings, another group recently showed that exposure of glial cells to $\mathrm{A} \beta$ activates nuclear factor kappa-B (NF- $\mathrm{kB}$ ) and induces miR-146a expression (Denk et al., 2015). Interestingly, inflammation-induced miR-146a expression has been reported in various other tissues/cell types, including immune cells (macrophage, dendritic, neutrophil, T cells and B cells) (Taganov et al., 2006; Lu et al., 2010; Boldin et al., 2011; Sun et al., 2015), hepatocytes (Sun et al., 2015), cardiomyocytes, bronchial epithelial cells, endothelial cells (HUVEC), orbital adipose tissue, as well as mesenchymal stem cells (Fu et al., 2017; Song et al., 2017). Together, these findings support the notion that inflammatory stimuli induces upregulation of miR-146a in a wide variety of cell types, and that this mechanism is likely involved in the immune response.

In addition to intracellular increases of miR-146a, we also found significant increases in miR146a expression in the EVs secreted from cells exposed to various inflammatory stimuli. EVmediated spread of disease may exacerbate the neuroinflammatory response (Rajendran et al., 2006) due to their ability to transfer functionally active proteins and RNAs to recipient cells (Ratajczak et al., 2006; Valadi et al., 2007). As inflammation-induced EVs are taken up by neighboring cells, their contents can be transferred into recipient cells and exert functional effects (i.e. miRNAs contained within EVs can repress protein translation in recipient cells) (Valadi et al., 2007). We observed a substantial upregulation of miR-146a in isolated EVs induced from exposure neuroinflammatory stimuli at levels much greater than control, and conclude that this is indicative of an increase in EVs derived from cultured cells. Taken together, our results are in agreement with others (Alexander et al., 2015; Song et al., 2017), and support the concept that EVs play a role in disease progression due to their ability to transfer aberrant proteins and RNAs to recipient cells.

In the $\mathrm{AD}$ brain, $\mathrm{A} \beta$ plaque deposition is considered a pathological hallmark of the disease and has been shown to induce inflammation. Previous work has observed the accumulation of A $\beta$ 
aggregates within microglia, as these brain immune cells attempt to degrade the insoluble protein aggregates to no avail (Paresce et al., 1997; Lee and Landreth, 2010; Krabbe et al., 2013; Baik et al., 2016). Additionally, complement factors and other inflammatory molecules have been observed within plaques themselves (Eikelenboom et al., 1989). It is clear that while the brain is trying to rid itself of these extracellular aggregates, an inflammatory response is being initiated within the surrounding tissue. Evidence from in vitro studies has shown that in response to A $\beta$ exposure, cultured human neuronal and glial cells upregulate expression of miR-146a due to the activation of NF-kB (Lukiw et al., 2008; Cui et al., 2010). Increased expression of miR-146a has been associated with another major characteristic of $\mathrm{AD}$, tau hyperphosphorylation, via the repression of rho-associated, coiled-coil containing protein kinase 1 (ROCK1) (Wang et al., 2016).

To better understand the interplay between inflammatory stimuli and decreased cellular bioenergetics, we investigated the potential effects of elevated levels of miR-146a on mitochondrial function and glycolysis. We show for the first time, that overexpression of miR146a leads to profound reductions in several parameters of mitochondrial function and glycolytic capabilities of primary mixed glial cells, which supports the notion that miR-146a exerts proinflammatory effects when it is significantly upregulated. Although we have not tested the direct effects of inflammatory stimuli on cellular bioenergetics, previous work has demonstrated that exposure to pro-inflammatory cytokines such as TNF- $\alpha$ and IL-1 $\beta$ leads to alterations in glucose metabolism and oxidative stress in astrocytes (Gavillet et al., 2008). In addition to classical inflammatory mediators, chronic exposure to soluble $A \beta$ peptides also results in impairment of energy homeostasis due to the decreased respiratory capacity of the mitochondrial electron transport chain; this may accelerate neuronal death (Rhein et al., 2009) as neurons are extremely dependent on aerobic metabolism and oxygen use. Despite a large reservoir of ATP, neurons are heavily dependent on aerobic metabolism and oxygen use, so reductions in ATP availability due to impaired glycolytic and/or mitochondrial function results in neuronal cell death (Goldberg and Choi, 1993; Lee et al., 2012b). 
Lukiw (2007) first demonstrated differential miRNA expression patterns in AD hippocampi, and later Cogswell et al. (2008) observed this phenomenon in specific brain regions and cerebrospinal fluid (CSF). Another group also found that miR-146a expression levels were significantly upregulated in both the CSF and hippocampi of AD patients (Denk et al., 2015). In addition to measuring miR-146a expression, we also correlated levels within specific brain regions (frontal and temporal cortices, and cerebellum) to Braak \& Braak staging (Braak and Braak, 1991b), and found significant positive correlations between these two factors. Additionally, expression levels of miR-146a were also positively correlated with CAA, which is characterized by deposition of $A \beta$ in the media and adventitia of cortical and leptomeningeal vessels (Salvarani et al., 2016). Aging and AD are established risk factors for developing CAA (Yamada, 2015), suggesting that not only parenchymal deposition of $A \beta$, but also intravascular accumulation, affects the expression of miR-146a during disease progression. These data indicate that multiple cell types may mediate the increase in miR-146a levels in $\mathrm{AD}$ and other neurological disease states. Brain hypometabolism is also significantly correlated with increased dementia in AD (Liang et al., 2008). Here, we have shown that within the temporal cortex of AD patients, as miR-146a expression increases, total mitochondrial protein decreases. Together these data support the hypothesis that inflammation-induced miR-146a overexpression contributes to brain hypometabolism in $\mathrm{AD}$.

In summary, we have shown that inflammatory stimuli expressed within the AD brain induce upregulation of miR-146a in a variety of cell types. Parallel findings in multiple brain cell types (neurons, astrocytes, microglia, and endothelial cells) and species (mouse, rat, and human) demonstrate that our observations may represent a common CNS inflammatory response mediated, in part, by miR-146a. Our functional data indicate that the overexpression of miR146a significantly impairs cellular bioenergetic function and may lead to the hypometabolic phenotype seen in clinical AD. These results suggest a novel pro-inflammatory mechanism of action for miR-146a in the neuroinflammatory etiology of AD and related dementias. 
Table 2.1: Fold change in miR-146a levels following transfection in primary mixed glial cells

Forty hours after transfection of vehicle or overexpression vector, total RNA was purified from primary mixed glial cells and their isolated EVs and miR-146a expression levels were assessed. All data were expressed as mean $\pm \mathrm{SEM} ; * \mathrm{p}<0.05$ compared to vector control.

\begin{tabular}{|cc|c|c|}
\cline { 2 - 4 } \multicolumn{1}{c|}{} & \multicolumn{2}{c|}{ miR-146a expression (relative fold change) } \\
\hline \multicolumn{2}{|c|}{ Transfection } & Intracellular & Extracellular Vesicle \\
\hline Vector & $4 \mu \mathrm{g} / \mathrm{ml}$ & $1.0 \pm 0.2$ & $1.0 \pm 0.0$ \\
\hline miR-146a & $2 \mu \mathrm{g} / \mathrm{ml}$ & $2.1 \pm 0.1$ & $53 \pm 19$ \\
\hline miR-146a & $4 \mu \mathrm{g} / \mathrm{ml}$ & $2.3 \pm 0.4$ & $116 \pm 20 *$ \\
\hline
\end{tabular}




\section{Figure legends}

Figure 2.1: Effects of pro-inflammatory stimuli on intracellular and EV miR-146a levels in different brain cell types. (A \& D) Murine hippocampal neurons (HT-22 cells), (B \& E) brain microglia (C8-B4 cells), or (C \& F) brain endothelial cells (bEnd.3) were exposed to either LPS $(1 \mu \mathrm{g} / \mathrm{ml})$ or TNF- $\alpha 1 \mathrm{ng} / \mathrm{ml}$ for 24 hours, followed by purification of cellular and EV miRNA. Intracellular miR-146a expression was greater in TNF- $\alpha$ exposed neurons (A) than in untreated, control cells ( $\mathrm{t}_{8.1}=-2.63, \mathrm{p} \leq 0.05$.) Similarly, TNF- $\alpha$ exposure also significantly increased miR146a expression in secreted neuronal EVs (D) when compared to EVs secreted from unexposed, control cells $\left(\mathrm{t}_{7.1}=7.06, \mathrm{p} \leq 0.0001\right.$.) Intracellular miR-146a expression was not significantly altered in microglial cells after exposure to LPS (B) when compared to unexposed, control cells $\left(\mathrm{t}_{2.1}=-8.39, \mathrm{p}=0.10\right.$.) Interestingly however, microglial EVs contained significantly elevated levels of miR-146a after exposure to LPS (E) when compared to unexposed control cells $\left(\mathrm{t}_{4}=\right.$ $53.58, \mathrm{p} \leq 0.0001)$. Intracellular miR-146a expression levels in brain endothelial cells exposed to LPS were significantly elevated when compared to control, while TNF- $\alpha$ exposure lead to significantly reduced miR-146a expression $(C)\left(\mathrm{F}_{2,6}=135.6, \mathrm{p} \leq 0.0001\right)$. EVs secreted from brain endothelial cells had significantly elevated miR-146a expression after LPS or TNF- $\alpha$ exposure, when compared to EVs secreted from unexposed, control cells $(F)\left(F_{2,6}=383.7, p \leq\right.$ $0.0001)$. Data are presented as means $\pm S E M$ with significance as $* p \leq 0.05, * * p \leq 0.01$, $* * * \mathrm{p} \leq 0.001$.

Figure 2.2: Effects of $\mathrm{A} \beta$ on intracellular and EV miR-146a levels in primary neurons and mixed glial cells. (A \& C) Rat primary neurons and (B \& D) mixed glial cells were exposed to 0, 250, and $500 \mathrm{nM}$ of $\mathrm{A} \beta$ for 24 hours, followed by purification of cellular and EV miRNA. Primary neurons exposed to both concentrations of $A \beta$ expressed significantly higher levels of intracellular miR-146a (A) when compared to unexposed, control cells $\left(\mathrm{F}_{2,6}=21.94, \mathrm{p} \leq 0.01\right)$. Similarly, EVs secreted from primary neurons exposed to both concentrations of $A \beta$ expressed significantly higher levels of miR-146a (C) when compared to EVs secreted from unexposed, control cells $\left(\mathrm{F}_{2,6}=147.4, \mathrm{p} \leq 0.0001\right)$. Primary microglia exposed to both concentrations of $\mathrm{A} \beta$ expressed significantly higher levels of intracellular miR-146a (B) when compared to unexposed, 
control cells $\left(\mathrm{F}_{2,6}=90.70, \mathrm{p} \leq 0.0001\right)$. Lastly, EVs secreted from primary microglia exposed to both concentrations of $A \beta$ expressed significantly higher levels of miR-146a (D) when compared to EVs secreted from unexposed, control cells $\left(\mathrm{F}_{2,6}=34.65, \mathrm{p} \leq 0.01\right)$. Data are presented as means \pm SEM with significance as $* \mathrm{p} \leq 0.05, * * \mathrm{p} \leq 0.01, * * * \mathrm{p} \leq 0.001$.

Figure 2.3: Functional effects of ectopically expressed miR-146a on oxidative phosphorylation in rat primary mixed glial cells. Mixed primary glial cells were transfected with a miR-146a overexpression vector for 40 hours, and mitochondrial function was assessed. ATP production (A) was significantly reduced in a miR-146a dose-dependent fashion $\left(\mathrm{F}_{1,71}=120, \mathrm{p} \leq 0.0001\right)$. Spare respiratory capacity $(\mathrm{B})$ was significantly reduced in a dose-dependent fashion $\left(\mathrm{F}_{1,72}=\right.$ 120.6, $\mathrm{p} \leq 0.0001)$. Maximal respiration was significantly reduced in a dose-dependent fashion $\left(\mathrm{F}_{1,72}=199.8, \mathrm{p} \leq 0.0001\right)$. Proton leak was significantly reduced in a dose-dependent fashion $\left(\mathrm{F}_{1,70}=77.66, \mathrm{p} \leq 0.0001\right)$. Data were analyzed by using regression models that showed a negative linear trend between the independent variable (miR-146a) and each dependent variable. All data are presented as means \pm SEM.

Figure 2.4: Overexpression of miR-146a reduces glycolysis in rat primary mixed glia. Rat primary mixed glia were transfected with a miR-146a overexpression vector and 40 hours after transfection, glycolysis was assessed. All data were analyzed using an ANOVA linear contrast. Glycolytic capacity (A) was significantly reduced in a miR-146a dose-dependent manner $\left(\mathrm{F}_{1,73}=85.23, \mathrm{p} \leq 0.0001\right)$. Glycolysis $(\mathrm{B})$ was significantly reduced in a dose-dependent fashion $\left(\mathrm{F}_{1,74}=150.7, \mathrm{p} \leq 0.0001\right)$. Glycolytic reserve $(\mathrm{C})$ was significantly reduced in a dosedependent manner $\left(\mathrm{F}_{1,71}=32.25, \mathrm{p} \leq 0.0001\right)$. Data were analyzed by using regression models that showed a negative linear trend between the independent variable (miR-146a) and each dependent variable. All data are presented as means \pm SEM.

Figure 2.5: miR-146a profile and correlation with pathology in human Alzheimer's disease (AD) regions compared to age-matched controls (AMC): temporal cortex (TC), frontal cortex (FC) and cerebellum (CB). Relative fold change of miR-146a levels in AD cases $(n=13)$ compared with $\operatorname{AMCs}(n=10)$ shows significant increases in the $\mathrm{TC}\left(\mathrm{A} ; \mathrm{t}_{11.2}=2.54, \mathrm{p} \leq 0.05\right)$ and $\mathrm{FC}\left(\mathrm{D} ; \mathrm{t}_{12.2}=\right.$ 
$2.29, \mathrm{p} \leq 0.05$ ) of $\mathrm{AD}$ brains when compared to AMCs. Of the AD brains, seven AD cases were diagnosed as Braak \& Braak (B\&B) stage VI, and six were diagnosed as stage III. The relationship of miRNA expression with B\&B stage (control, 0 vs. 3 vs. 6) was also assessed to determine $B \& B$ stage specific miR-146a expression in human brains and found significant increases within the TC (B; $\left.\mathrm{F}_{2,19}=9.91, \mathrm{p} \leq 0.01\right)$, FC (E; $\left.\mathrm{F}_{2,20}=7.05, \mathrm{p} \leq 0.01\right)$ and $\mathrm{CB}\left(\mathrm{H} ; \mathrm{F}_{2,21}\right.$ $=7.19, \mathrm{p} \leq 0.01)$. Correlation of miR-146a expression with $\mathrm{B} \& \mathrm{~B}$ stage or amyloid angiopathy (AA) was calculated by using Spearman's rho $(\rho)$ and showed a significant correlation of miR146a with B\&B stage: (C) TC, $\rho=0.68, p \leq 0.001$; (F) FC, $\rho=0.61, p \leq 0.01$; (I) $C B, \rho=0.56, p \leq$ 0.01 and AA: (C) TC, $\rho=0.53, p \leq 0.05$; (F) FC, $\rho=0.48, p \leq 0.05$; (I) $\mathrm{CB}, \rho=0.49, \mathrm{p} \leq 0.05$ ). Data are presented as means \pm SEM with significance as ${ }^{*} \mathrm{p} \leq 0.05,{ }^{* *} \mathrm{p} \leq 0.01,{ }^{* * *} \mathrm{p} \leq 0.001$.

Figure 2.6: Mitochondrial protein levels are inversely correlated with miR146a gene expression. (A) Western blot of representative $\mathrm{AD}$ and $\mathrm{AMC}$ temporal cortex shows levels of the mitochondrial protein, voltage-dependent anion channel (VDAC, $35 \mathrm{kD}$ ), and the cytosolic housekeeping protein, $\beta$-actin (46 kD). (B) VDAC levels were normalized to beta-actin for each sample (VDAC/ $\beta$-actin). The correlation between the VDAC/ $\beta$-actin ratio and miR-146a expression revealed an inverse releationship between total mitochondrial proteins and miR146a expression (Spearman's $\rho=-0.80, \mathrm{p} \leq 0.01$ ). 

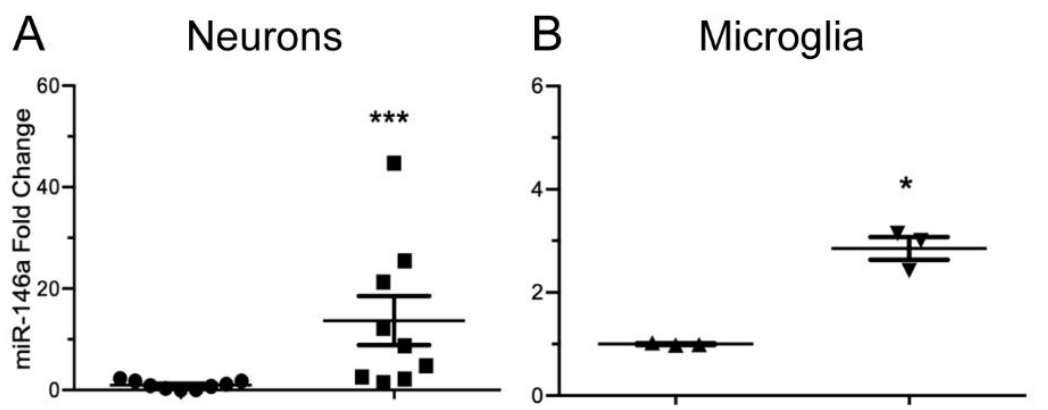

C Brain Endothelium
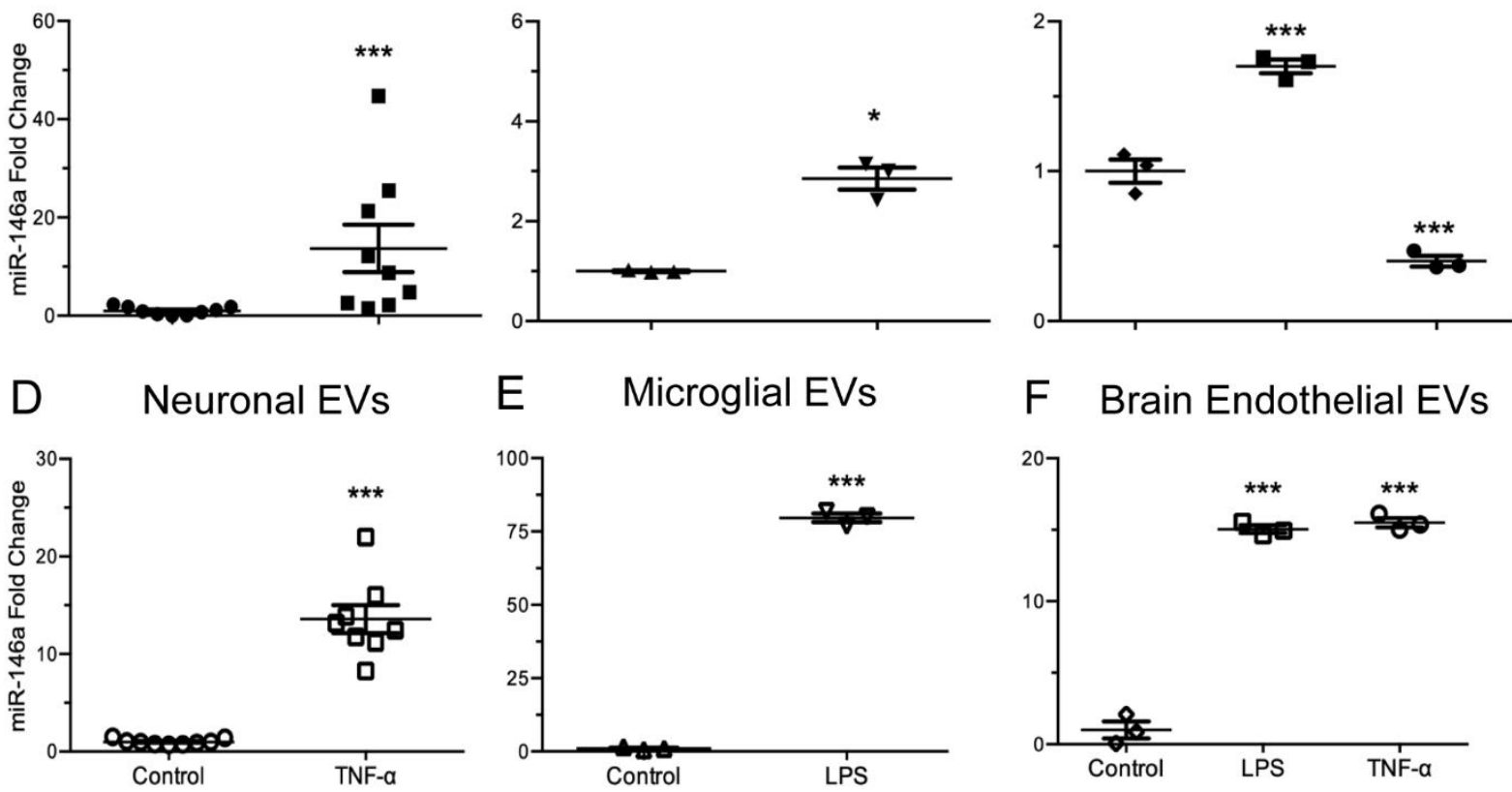

Figure 2.1

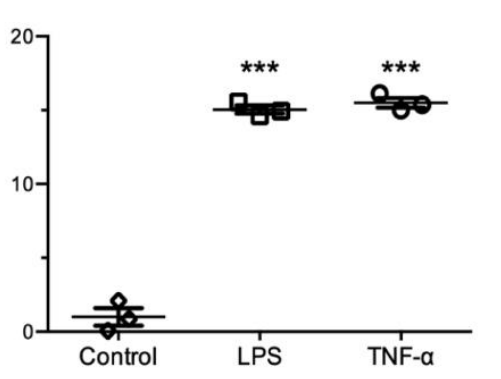



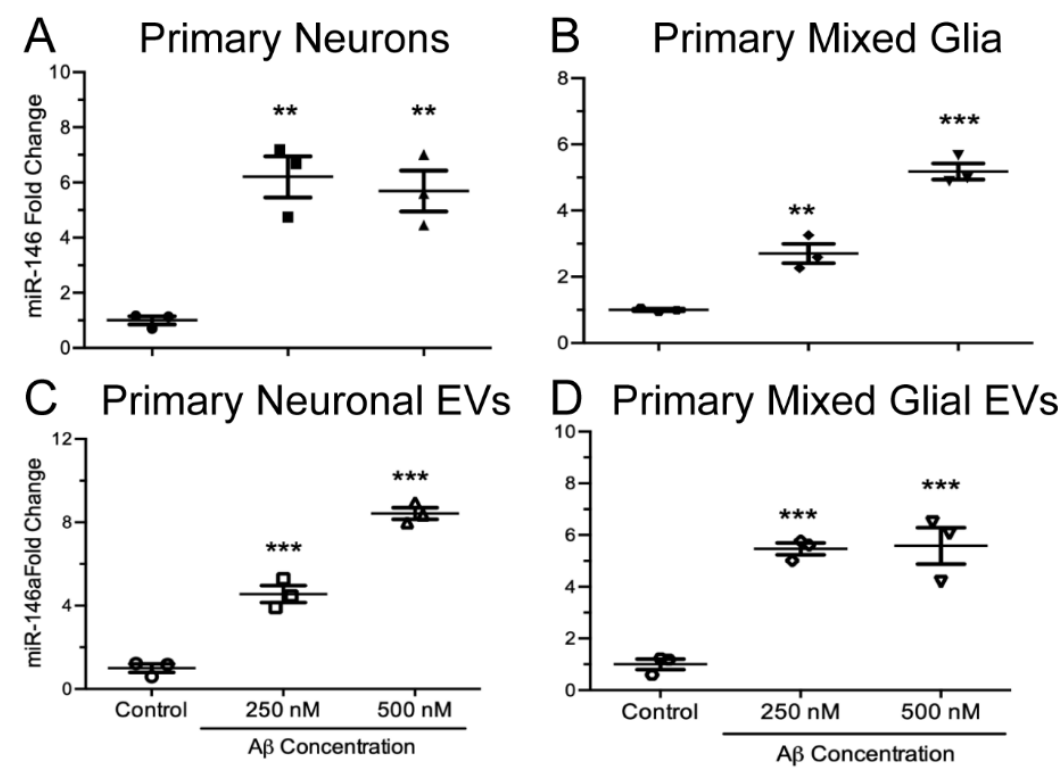

Figure 2.2 

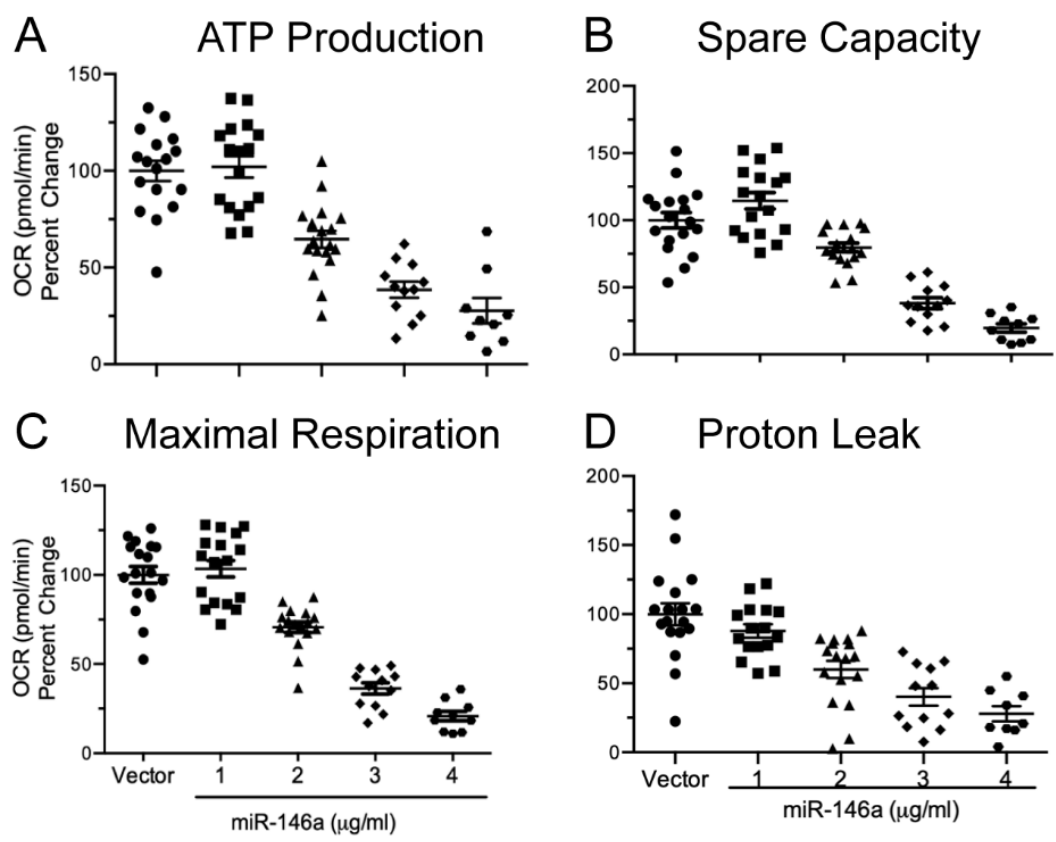

Figure 2.3 

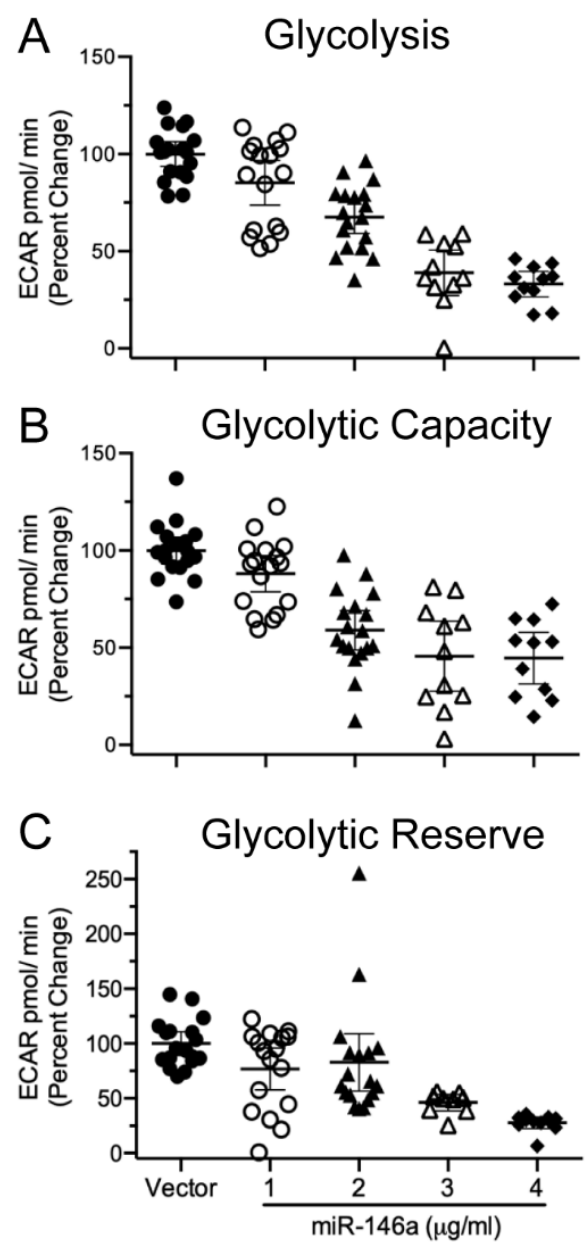

Figure 2.4

52 


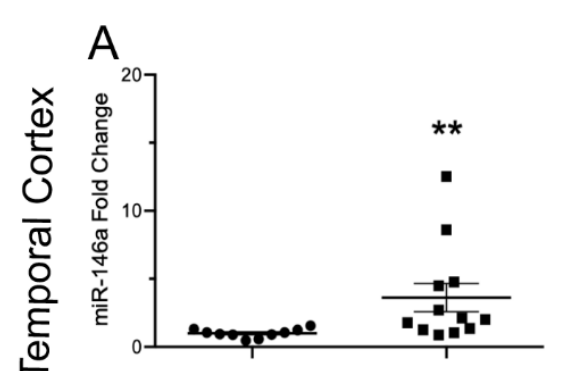

D
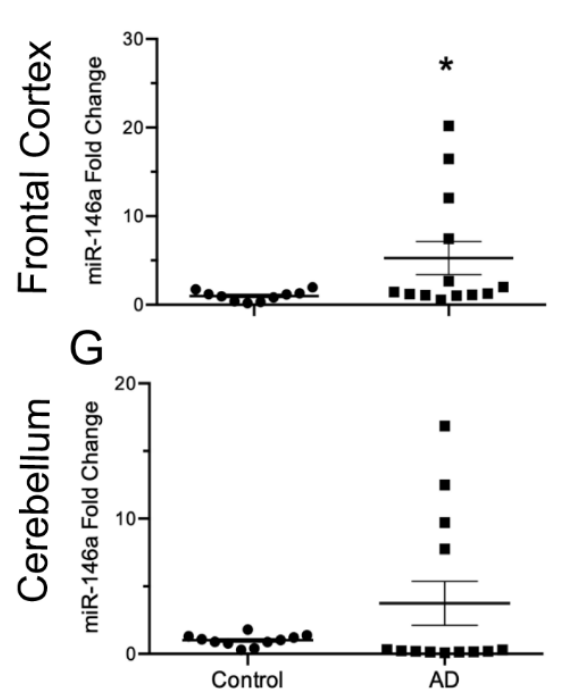
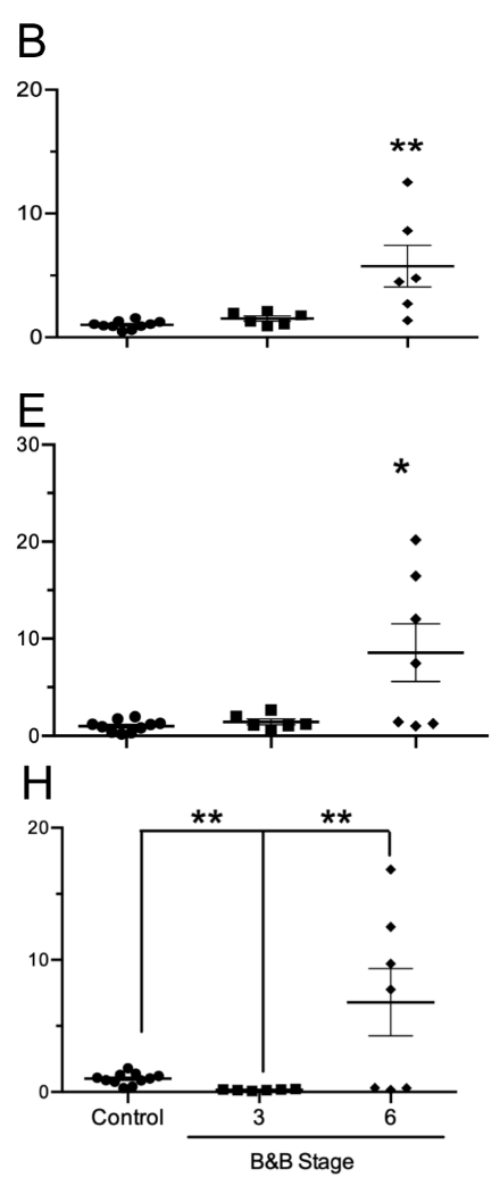

C $\rightarrow$ B\&B Stage
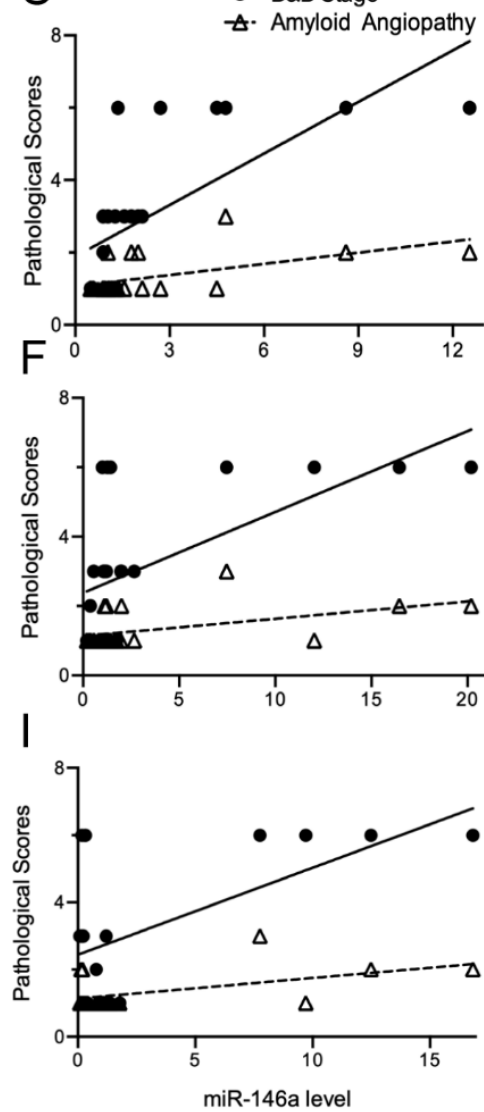

Figure 2.5 
A
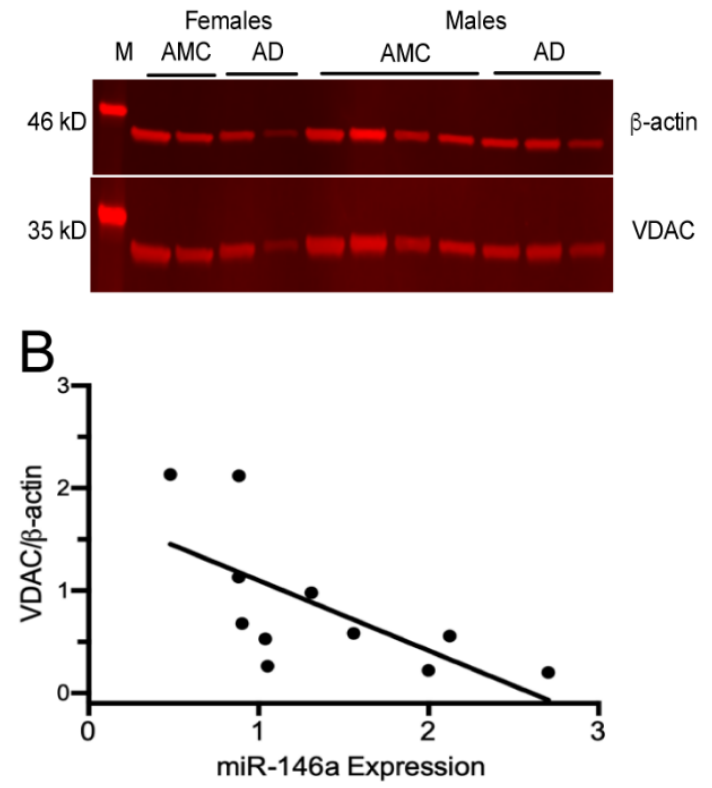

Figure 2.6 


\section{Chapter 3}

\section{Extracellular vesicles secreted in response to cytokine exposure increase mitochondrial oxygen consumption in recipient cells}

\section{Ashley E. Russell, Sujung Jun, Saumyendra Sarkar, Werner J. Geldenhuys, Sara E. Lewis, Stephanie L. Rellick, and James W. Simpkins}

Author Contribution: AR, SJ, SS, WG, and JS designed studies; AR and WG performed research; AR, SJ, SL, and SR analyzed data; AR and JS wrote the paper.

Russell AE, Jun S, Sarkar S, Geldenhuys WJ, Lewis SE, Rellick SL, Simpkins JW (2019) Extracellular vesicles secreted in response to cytokine exposure increase mitochondrial oxygen 


\section{consumption in recipient cells. Frontiers in Neuroscience. In press.}

\subsection{Abstract}

Extracellular vesicles (EVs) are small, membrane-bound nanoparticles released from most, if not all cells, and can carry functionally active cargo (proteins, nucleic acids) which can be taken up by neighboring cells and mediate physiologically relevant effects. In this capacity, EVs are being regarded as novel cell-to-cell communicators, which may play important roles in the progression of neurodegenerative diseases, like Alzheimer's disease. Aside from the canonical physical hallmarks of this disease (amyloid $\beta$ plaques, neurofibrillary tangles, and widespread cell death), $\mathrm{AD}$ is characterized by chronic neuroinflammation and mitochondrial dysfunction. In the current study, we sought to better understand the role of tumor necrosis factor-alpha (TNF- $\alpha$ ), known to be involved in inflammation, in mediating alterations in mitochondrial function and $\mathrm{EV}$ secretion. Using an immortalized hippocampal cell line, we observed significant reductions in several parameters of mitochondrial oxygen consumption after a 24-hour exposure period to TNF- $\alpha$. In addition, after TNF- $\alpha$ exposure we also observed significant upregulation of two miRNAs (miR-34a and miR-146a) associated with mitochondrial dysfunction in secreted EVs. Despite this, when naïve cells are exposed to EVs isolated from TNF- $\alpha$ treated cells, mitochondrial respiration, proton leak, and reactive oxygen species (ROS) production are all significantly increased. Collectively these data indicate that a potent proinflammatory cytokine, TNF- $\alpha$, induces significant mitochondrial dysfunction in a neuronal cell type, in part via the secretion of EVs, which significantly alter mitochondrial activity in recipient cells. 


\subsection{Introduction}

Alzheimer's disease (AD) is a chronic neurodegenerative disease characterized by amyloid $\beta$ (A $\beta$ ) plaques, neurofibrillary tangles, neuroinflammation, and mitochondrial dysfunction (WyssCoray and Rogers, 2012). Extracellular A $\beta$ plaques activate microglia, prompting secretion of immunomodulatory molecules, including tumor necrosis factor-alpha (TNF- $\alpha$ ) (Hanisch, 2002). Elevated levels of this cytokine have been detected in the plasma (Fillit et al., 1991; Zuliani et al., 2007), cerebrospinal fluid (CSF) (Tarkowski et al., 2003), and brains (Sharma et al., 2012) of AD patients when compared to healthy, age matched controls (AMCs). Further, several studies have implicated TNF- $\alpha$ as a key player in the formation of amyloid $\beta$ (A $\beta$ ) plaques (Blasko et al., 2000; Liao et al., 2004; Yamamoto et al., 2007), suggesting that this cytokine may participate in a vicious cycle of $\mathrm{AD}$ progression. TNF- $\alpha$ activates the transcription factor NF- $\kappa \mathrm{B}$ (Schütze et al., 1995), which induces the transcription of many genes, including microRNAs (miRNAs); these small, non-coding RNAs function as gene repressors by base-pairing with complementary mRNAs and inhibiting protein translation, or accelerating the de-adenylation of the poly-A tail (Krek et al., 2005; Huntzinger and Izaurralde, 2011). Changes in expression of various miRNAs have been associated with inflammation and may influence AD progression.

Previous work from our laboratory assessed brain region-specific changes in miRNA expression, and observed significant upregulation of miR-34a and miR-146a in AD patients' temporal cortices, when compared to AMCs (Sarkar et al., 2016). Promotor analysis revealed that various components of the NF- $\kappa \mathrm{B}$ family bind to the promotor region of both miR-34a (Sarkar et al., 2016) and miR-146a (Taganov et al., 2006), suggesting that the activation of pro-inflammatory pathways could lead to increased transcription of these miRNAs. Interestingly, these miRNAs target several mRNAs, which encode for proteins of the mitochondrial electron transport chain (ETC) (Dasgupta et al., 2015; Sarkar et al., 2016). Indeed, overexpression of miR-146a in primary microglial cells (Jun et al., unpublished observation) or miR-34a in primary neurons (Sarkar et al., 2016) significantly reduced mitochondrial function, and repressed expression of all of the associated mitochondrial proteins that were assessed (Sarkar et al., 2016). Inflammationinduced upregulation of NF- $\kappa$ B may lead to aberrant expression of miR-34a and $-146 \mathrm{a}$ and lead 
to translational repression of ETC proteins, thus contributing to the widespread reductions in glucose metabolism and dysregulated mitochondrial function observed in AD brains.

Intercellular communication allows for the transfer of information from one cell to another. The secretion of extracellular vesicles (EVs) affords the opportunity for cells to send important signaling molecules out into the extracellular environment to convey vital messages to neighboring cells (Harding et al., 1983; Pan et al., 1985; Valadi et al., 2007; Tkach and Théry, 2016). As EVs are encapsulated by a lipid bilayer, their contents (including proteins, DNA, and RNA) are protected from degrading enzymes, allowing for delivery of functional cargo. During periods of neuroinflammation, concentrations of circulating EVs have been shown to be significantly upregulated, and within the context of $\mathrm{AD}$, EVs have been shown to confer noxious molecules, including A $\beta$ (Rajendran et al., 2006; Sardar Sinha et al., 2018), to recipient cells and thereby participate in the spreading of AD pathology and neuroinflammation.

Inasmuch as neuroinflammation clearly occurs in affected individuals, and likely occurs years prior to onset of clinical symptoms, it is difficult to model in vitro, the effects of long-term inflammation. In the current study, we sought to characterize the role of acute TNF- $\alpha$ exposure on phenotypes seen in $\mathrm{AD}$, including mitochondrial dysfunction, increased miRNA expression (e.g. miR-34a and miR-146a) (Sarkar et al., 2016), and altered EV secretion in an immortalized hippocampal cell line (HT-22). Here we demonstrate that direct exposure to TNF- $\alpha$ for 24 -hours significantly reduces mitochondrial oxygen consumption, induces cellular death, and induces differential expression of miR-34a and miR-146a both within cells, and in secreted EVs. We also assessed the functional outcomes of exposing TNF- $\alpha$ naïve cells to TNF- $\alpha$-induced EVs and found significant increases in mitochondrial oxygen consumption, proton leak, and reactive oxygen species (ROS) production.

\subsection{Materials and Methods}

\section{Cell culture}


The HT-22 immortalized mouse hippocampal cells were obtained from the Salk Institute for Biological Research (La Jolla, CA, USA). HT-22 cells were cultured in Hyclone Dulbecco's modified Eagle's medium (DMEM)/high glucose (Fisher Scientific, Waltham, MA, USA) with $10 \%$ fetal bovine serum (FBS) (Atlanta Biologicals, Flowery Branch, GA, USA) and 1\% penicillin/streptomycin (Fisher Scientific). When cells were at least $80 \%$ confluent, they were trypsinized and spun down at $325.5 \mathrm{x}$ g for 3 minutes. Cells were counted with a Nexcelom Bioscience Cellometer AutoT4 (Lawrence, MA, USA). Cell passages 5-18 were used for all experiments.

\section{Cytokine reconstitution and exposure}

Recombinant mouse TNF- $\alpha$ was purchased from R\&D Systems (Minneapolis, MN, USA) and reconstituted at $100 \mu \mathrm{g} / \mathrm{ml}$ in phosphate-buffered saline (PBS) containing $0.1 \%$ bovine serum albumin. Dilutions were made in Hyclone DMEM/high glucose with 10\% exosome-depleted FBS (Fisher Scientific), and 1\% penicillin/streptomycin to obtain concentrations of 0.1, 1, and 10 $\mathrm{ng} / \mathrm{ml}$. The 24-hour time point for TNF- $\alpha$ exposure was chosen as preliminary data suggested that shorter exposure period did not result in mitochondrial dysfunction (data not shown). Longer exposure periods were not tested due to the potential complication of TNF- $\alpha$-induced neurotoxicity on cell number, which could affect readouts of all of the assessed parameters in this study.

\section{EV isolation from cell culture media}

Conditioned media was collected after a 24-hour exposure to TNF- $\alpha$ and filtered through a Millex-AA Syringe Filter Unit, $0.80 \mu \mathrm{m}$ (Millipore Sigma, Burlington, MA, USA) to remove cellular debris. EV isolation was performed as per the manufacturer's instructions using either the ExoRNeasy Serum Plasma Maxi Kit (Qiagen, Germantown, MD) utilized for RNA purification from EVs, or the ExoEasy Maxi Kit (Qiagen) for all other EV applications.

Briefly, 1 volume of filtered media was mixed with 1 volume of buffer XBP and then placed on a spin column and centrifuged at $500 \mathrm{x} g$ for 1 minute. The flow-through was discarded and the column was washed with $10 \mathrm{ml}$ buffer XWP and centrifuged at $>3000 \mathrm{xg}$ for 5 minutes. The 
column was then transferred to a new collection tube and the EVs were eluted with $700 \mu \mathrm{l}$ QIAzol for downstream RNA purification, or $400 \mu \mathrm{l}$ buffer XE for all other EV applications.

\section{Particle size distributions and concentrations}

To determine if changes in EV concentrations after exposure to TNF- $\alpha$ could account for alterations in mitochondrial function, EVs were isolated from HT-22 cell conditioned media using the ExoEasy Maxi Kit (as described above) and profiled with the NanoSight NS300 (Malvern, Westborough MA, USA). In the final isolation step using the ExoEasy Maxi Kit, EVs were eluted from the spin column membrane in $400 \mu \mathrm{XE}$ Buffer. Suspended EVs were diluted 1:200 in sterile filtered PBS for injection into the NanoSignt NS300 unit. Capture and analysis settings were manually set according to the manufacturer's instructions. Particles were visualized using laser light scattering to quantify nanoparticles $(10-1000 \mathrm{~nm})$ moving under Brownian motion as they pass through the flow chamber. The Nanoparticle Tracking Analysis (NTA) software generates particle size distributions and concentrations based on an analysis of both Brownian motion and light scattering observed from tracked particles.

\section{EV marker dot blot}

The presence of several EV markers was assessed to ensure that isolated particles from control or TNF- $\alpha$ exposure groups were indeed EVs. Protein concentration was measured using a microBCA kit (Fisher). BSA standards were prepared in the same solution EVs were eluted in (XE Buffer). EVs were diluted in 2\% sodium dodecyl sulfide (SDS) at a ratio of 1:10 to a final volume of $150 \mu \mathrm{l}$, and transferred to a 96 well clear bottom assay plate. The standard curve was prepared using $150 \mu \mathrm{l}$ of each, in duplicate. Working reagent was prepared in a 25: 24: 1 ratio of reagents $\mathrm{A}, \mathrm{B}$, and $\mathrm{C}$, respectfully. $150 \mu \mathrm{l}$ working reagent was added to each of the standards and diluted EV samples, and incubated at room temperature for 2 hours on a shaker. The plate was read using a BioTek Synergy H1 Hybrid reader at $562 \mathrm{~nm}$ absorbance.

The Exo-Check Exosome Antibody Array (System Biosciences) was used to detect the presence of several EV markers as per the manufacturer's instructions. Briefly, from either control or TNF- $\alpha$ induced EVs, $200 \mu \mathrm{g}$ protein was incubated with $600 \mu \mathrm{l}$ Exosome Lysis buffer and 
vortexed. Next, the lysate mixture was combined with $9.4 \mathrm{~mL}$ Exosome Array Binding buffer and was then added to a pre-wet antibody array membrane and incubated overnight at $4{ }^{\circ} \mathrm{C}$ on a shaker. The following day the membrane was washed and $10 \mathrm{~mL}$ Detection buffer was added to the membrane and incubated for 2 hours on a shaker. Next, the membrane was washed $3 x$ and 2 mL SuperSignal West Femto Chemi-luminescent Substrate (Fisher) developer solution was added and the membrane was imaged.

\section{Mitochondrial functional assessment}

HT-22 cells were seeded at 5k per well in an XFe96 cell culture microplate (Agilent, Santa Clara, CA) for 24 hours. Media was gently aspirated from the cells and replaced with $100 \mu$ Hyclone DMEM/high glucose, supplemented with 10\% exosome-depleted FBS and 1\% penicillin/streptomycin, the various concentrations of TNF- $\alpha$, or isolated TNF- $\alpha$-induced EVs. For EV exposure, EVs were isolated from the media of HT-22 cells exposed to varying concentrations of TNF- $\alpha(0,0.1,1$, and $10 \mathrm{ng} / \mathrm{ml})$ for 24 hours. As described above, EVs were isolated from $20 \mathrm{ml}$ media of cytokine-exposed HT-22 cells and eluted from spin columns in 400 $\mu \mathrm{l}$ buffer XE. Assuming a high yield of recovery from a starting volume of $20 \mathrm{ml}$ conditioned cell culture media, EVs eluted in $400 \mu \mathrm{l}$ buffer XE would be concentrated 50x. To mimic in vitro EV concentrations from each cytokine exposure condition, EVs were diluted 50x in DMEM/high glucose, supplemented with $10 \%$ exosome-depleted FBS and $1 \%$ penicillin/streptomycin (i.e. 2 $\mu \mathrm{l}$ EVs suspended in buffer XE were diluted into $98 \mu 1$ media per well). Various parameters of mitochondrial function were assessed using a Mito Stress kit, with the XF 96 Analyzer. Using Wave 2.4.0 Desktop software, all mitochondrial assessments were calculated with the Agilent Seahorse XF Cell Mito Stress Test Report Generator. Non-mitochondrial oxygen consumption rate (OCR) was calculated as the minimum rate measurement after rotenone/antimycin $\mathrm{A}$ injection. Basal respiration was calculated by subtracting non-mitochondrial OCR from the last rate measurement before oligomycin injection. Maximal respiration was calculated by subtracting non-mitochondrial OCR from the maximum rate measurement after trifluorocarbonylcyanide phenylhydrazone (FCCP) injection. Proton leak was calculated by subtracting non-mitochondrial OCR from the minimum rate measurement after Oligomycin injection. ATP production was calculated by subtracting the minimum rate measurement after 
Oligomycin injection from the last rate measurement before Oligomycin injection. Lastly, spare respiratory capacity was calculating by subtracting basal respiration from maximal respiration. All measurements are displayed percent changes from control. All wells with a basal respiratory rate below $40 \mathrm{pmol} / \mathrm{min}$ were excluded from analysis.

While glycolysis was not directly measured, data on the extracellular acidification rate (ECAR) is an additional measurement collected while completing the Mito Stress test. From these measurements, basal levels of glycolytic activity can be determined. To determine basal glycolysis, the third measurement, prior to the injection of oligomycin, was used. For cells treated with TNF- $\alpha$, the average ECAR reading was calculated, and was used to calculate percent of control for each of the TNF- $\alpha$ treatment groups $(0,0.1,1$, and $10 \mathrm{ng} / \mathrm{ml})$. For cells treated with EVs derived from TNF- $\alpha$ exposed cells, the cells treated with the XE buffer served as the control, and percent of control was calculated in the same way in each of the treatment groups $(0$, $0.1,1$, and $10 \mathrm{ng} / \mathrm{ml})$.

\section{Lactate dehydrogenase assay}

Cell death after cytokine or EV exposure was assessed using the Pierce ${ }^{\mathrm{TM}}$ lactate dehydrogenase (LDH) Cytotoxicity Assay (Fisher Scientific) kit. Reaction substrates were prepared as per the manufacturer's instructions. LDH assay was performed with the media from the XFe96 cell culture microplate. Forty-five minutes prior to the end of the 24-hour exposure period, $10 \mu 110 \mathrm{x}$ lysis buffer was added to one control well, and the plate was placed back in the incubator. At the conclusion of the exposure period, $50 \mu \mathrm{l}$ of media was carefully removed from each well and transferred to a new 96 well clear bottom assay plate. The well exposed to 10x lysis buffer was excluded from the mitochondrial functional assessment. Next, $50 \mu \mathrm{l}$ of the LDH reaction mixture was added to each well, and the plate was incubated for 30 minutes at room temperature, protected from light. The reaction was stopped by adding $50 \mu \mathrm{l}$ of LDH stop solution to each sample. The plate was read using the BioTek Synergy H1 Hybrid reader (BioTek, Winooski, VT) at absorbance of $490 \mathrm{~nm}$ and $680 \mathrm{~nm}$. Cell death was calculated as a percentage of total death, relative to maximal cell death induced by treatment with the 10x lysis buffer. 


\section{Intracellular and EV miRNA analysis}

Cells were seeded at $250 \mathrm{k}$ per $10 \mathrm{~cm}$ cell culture dish and left to reach $\sim 80 \%$ confluency over the course of 48 hours. Media was gently aspirated from cells, and replaced by $10 \mathrm{ml}$ of Hyclone DMEM/high glucose, supplemented with 10\% exosome-depleted FBS and 1\% penicillin/streptomycin, or the various concentrations of TNF- $\alpha$. After a 24 hour exposure period the media was collected for EV isolation, and cell lysates were collected using a Cell Lifter (Corning, Corning, NY, USA), washed with PBS, and pelleted by centrifugation at $325.5 \mathrm{x}$ g for 3 minutes, followed by the addition of $700 \mu \mathrm{l}$ QIAzol Lysis Reagent for RNA purification (Qiagen).

RNA was purified from EVs eluted in $700 \mu \mathrm{l}$ QIAzol by using the exoRNeasy Serum Plasma Maxi Kit (Qiagen). For all EV samples, $3.5 \mu 1$ miRNeasy Serum/Plasma Spike-In Control ( $C$. elegans miR-39; at a concentration of $1.6 \times 10^{8}$ copies/ $\mu 1$ ) was added to each sample, followed by the addition of $90 \mu \mathrm{l}$ chloroform and a 3 minute incubation. Next the samples were centrifuged for 15 minutes at $12,000 \mathrm{xg}$ at $4^{\circ} \mathrm{C}$. The upper aqueous phase $(300 \mu \mathrm{l})$ was transferred to a new collection tube and mixed with $600 \mu 100 \%$ ethanol. The solution was then placed on an RNeasy MinElute spin column and centrifuged at 12,000 x g for 15 seconds. The flow through was discarded and $700 \mu \mathrm{l}$ buffer RWT was added to the spin column and centrifuged at $12,000 \mathrm{x}$ g for 15 seconds. Two washes of $500 \mu \mathrm{l}$ buffer RPE were then performed, the first lasting 15 seconds and the second lasting 2 minutes. The RNeasy MinElute spin column was then placed in a new collection tube and spun at 12,000 x g for 5 minutes to dry the column membrane. Lastly, the column was placed in another collection tube and $28 \mu 1$ RNase-free water was added to the center of the membrane and incubated for 1 minute, and then centrifuged at $12,000 \times \mathrm{g}$ for 1 minute ending with the purified RNA eluted in the collection tube. Cellular RNA was purified using the miRNeasy Mini Kit (Qiagen) as per the manufacturer's instructions, similar to the purification process for EVs, as described above.

RNA concentrations for each sample were measured using Nano drop 2000 spectrophotometer (Thermo Scientific). For cDNA synthesis, $0.75 \mu \mathrm{g}$ total RNA containing microRNAs was reverse transcribed using the miScript II RT kit (Qiagen). A $10 \mu$ total volume reaction mix was 
made using $2 \mu \mathrm{l}$ 5x miScript HiSpec Buffer, $1 \mu$ 10x miScript Nucleics Mix, $1 \mu 1$ miScript Reverse Transcriptase Mix, and $6 \mu 1$ Template RNA/nuclease-free water. Prior to use for qRTPCR, cDNA was diluted in nuclease-free water, at a ratio of 1:10.

Previous work from our laboratory indicates that of a panel of miRNAs assessed, only miR-34a and miR-146a showed disease-stage related increases in expression in both human AD and triple transgenic AD mouse (3xTgAD) brains. Therefore, expression of miR-34a and miR-146a were profiled for both EV and intracellular levels using target specific miScript primer assays and the miScript SYBR ${ }^{\circledR}$ Green PCR kit (Qiagen) (5 $\mu$ l SYBR ${ }^{\circledR}$ Green; $1 \mu$ Universal Primer; $1 \mu 1$ Target Primer; $3 \mu$ diluted cDNA). Quantitative RT-PCR reactions were performed in triplicate for each sample, using the CFX384 Touch ${ }^{\mathrm{TM}}$ Real-Time PCR Detection System (Bio-Rad, Hercules, CA, USA) for 45 cycles as follows: $15 \mathrm{sec}$ at $94{ }^{\circ} \mathrm{C}, 30 \mathrm{sec}$ at $55^{\circ} \mathrm{C}, 30 \mathrm{sec}$ at $70{ }^{\circ} \mathrm{C}$. Negative control reactions were included as wells containing only master mix and nuclease-free water (no template cDNA). All miRNA specific primers were miScript Primer Assays (Qiagen); Hs_RNU6-2_11 (MS00033740), Ce_miR-39_1 (219610), Hs_miR-34a (MS00003318), and Hs_miR-146a (MS00003535). The expression levels of target genes in EVs were standardized against those of the spike-in control, c.elegans miR-39 gene, detected in identical cDNA samples, while the expression levels of target genes in cell lysates was standardized against RNU6. Quantification of PCR amplified microRNA specific cDNA was done by comparative cycle threshold CT method $\left(2^{-\Delta \Delta C T}\right)$.

\section{EV uptake via naïve cells}

To determine if naïve HT-22 cells have the capacity to take up cytokine-induced EVs, cells were incubated with EVs labeled with Exo-Red Exosome RNA Fluorescent Label (System Biosciences, Palo Alto, CA). EVs isolated from all TNF- $\alpha$ exposure groups were labeled as per the manufacturer's protocol. Briefly, $\sim 100 \mu \mathrm{g}$ protein of isolated EVs were incubated with $50 \mu \mathrm{l}$ Exo-Red for 10 minutes at $37^{\circ} \mathrm{C}$. ExoQuick-TC reagent $(100 \mu \mathrm{l})$ was then added to the labeled EV suspension and mixed by gentle inversion. This solution was incubated on ice for 30 minutes, followed by centrifugation at 14,000 rpm for 3 minutes. The supernatant was removed and the EV pellet was resuspended in $500 \mu \mathrm{l}$ PBS. Next, $100 \mu$ labeled EVs were added to naïve 
HT-22 cells in a 6-well dish ( $\sim 80 \%$ confluent), and imaged with the Nikon Swept Field Confocal microscope (Nikon, Melville, NY) approximately 10 minutes after addition to naïve cells.

\section{Mitochondrial membrane potential assay}

Mitochondrial membrane potential ( $\Psi \mathrm{m})$ was measured using the tetramethylrhodamine, ethyl ester (TMRE) (Abcam, Cambridge, United Kingdom) reagent. Cells were grown in a 96 well clear bottom black plate and exposed to TNF- $\alpha$ induced vesicles as described for the Seahorse assay. At the end of the experiment, media was gently aspirated from the wells and replaced by $100 \mu 1$ of $60 \mathrm{nM}$ TMRE in cell culture media, and incubated at $37^{\circ} \mathrm{C}$ for 20 minutes, protected from light. Cells were gently washed three times with warmed HBSS and fluorescence was measured on a Synergy HT multimodal plate reader with excitation $549 \mathrm{~nm}$ and emission 575 nm.

\section{Mitochondrial superoxide assay}

The levels of mitochondrial superoxide in cells were determined using the MitoSOX $^{\mathrm{TM}}$ reagent (Fisher). Cells were grown in a 96 well clear bottom black plate and exposed to TNF- $\alpha$ induced vesicles as described for the Seahorse assay. At the end of the experiment, media was gently aspirated from the wells and replaced by $100 \mu \mathrm{l}$ of $5 \mu \mathrm{m}$ MitoSox in cell culture media and incubated for 10 minutes at $37^{\circ} \mathrm{C}$, protected from light. Cells were gently washed three times with warmed HBSS and fluorescence was measured on a Synergy HT multimodal plate reader with excitation $510 \mathrm{~nm}$ and emission $580 \mathrm{~nm}$.

\section{Hydrogen peroxide assay}

The hydrogen peroxide assay was performed using the Amplex Red Kit (Invitrogen). Cells were grown in a 96 well clear bottom black plate, and exposed to TNF- $\alpha$ induced vesicles as described for the Seahorse assay. At the end of the experiment, $50 \square \mathrm{L}$ of the Amplex Red reagent was added to the media in the plate without washing, and incubated for $30 \mathrm{~min}$ at room temperature. The fluorescence of the Amplex Red reagent was measured on a Synergy HT multimodal plate reader with excitation $530 \mathrm{~nm}$ and emission $590 \mathrm{~nm}$. 


\section{Statistical analyses}

All biological experiments were repeated at least 3 times with $n=3-16$ plates/wells per treatment. Results from the experiments are reported as means \pm SEM. All quantitative data were assessed for significance using a one-way ANOVA with Dunnett's post hoc test. All results were analyzed by GraphPad Prism 8.0 software (GraphPad Software, La Jolla, CA). A p value < 0.05 was used to establish significance; significance presented as $* \mathrm{p}<0.05, * * \mathrm{p}<0.01$, $* * * \mathrm{p}<0.001$, and $* * * * \mathrm{p}<0.0001$

\subsection{Results}

TNF- $\alpha$ induces mitochondrial dysfunction and cellular death

A Seahorse mitochondrial stress test was conducted to assess mitochondrial function in HT-22 cells exposed to TNF- $\alpha$ for 24 hours. Results indicate that TNF- $\alpha$ exposure reduced basal and maximal respiration, ATP production, and spare capacity in a dose-dependent manner, with significant reductions occurring at the two highest concentrations ( 1 and $10 \mathrm{ng} / \mathrm{ml}$ ) compared to unexposed control cells (Figures 1A-D). These same concentrations of TNF- $\alpha$ induced significant proton leak (Figure 1E), which can reduce the $\Psi \mathrm{m}$, and uncouple oxidative phosphorylation (Terada, 1990; Jastroch et al., 2010). Significant, dose-dependent decreases in

basal glycolysis were also detected as a result of TNF- $\alpha$ exposure (Figure 1F). Exposure to all concentrations of TNF- $\alpha$ lead to cell death as shown by significant increases in lactate dehydrogenase (LDH) present in the conditioned culture media (Figure $1 \mathrm{G})$. In view of the apparent cell death induced by increasing doses of TNF- $\alpha$ (Figure $1 \mathrm{G}$ ), the observed decline in oxidative phosphorylation (Figures 1A-D) and glycolysis (Figure 1F) may be caused by the loss of cells in the assay.

TNF- $\alpha$ exposure induces EV secretion, but not in a dose-dependent fashion We next assessed the ability of TNF- $\alpha$ exposure to induce EV secretion in this hippocampal cell line. Vesicles isolated from the conditioned media of TNF- $\alpha$ exposed cells were profiled using the NanoSight NS300. Interestingly, TNF- $\alpha$ exposure lead to increased EV particle number, 
however this effect does not appear to be dose-dependent (Figures 2A, B). Exposure to low (0.1 $\mathrm{ng} / \mathrm{ml})$ and high (10 ng/ml) concentrations of TNF- $\alpha$ significantly increased particle concentration, whereas exposure to $1 \mathrm{ng} / \mathrm{ml} \mathrm{TNF}-\alpha$ did not lead to particle concentrations above control. Additionally, TNF- $\alpha$ exposure did not alter vesicle size, as all EV size distributions were between $~ 80-300 \mathrm{~nm}$ in diameter, consistent with what has been shown in the literature (van Niel et al., 2018).

As recommended by the International Society for Extracellular Vesicles (Lötvall et al., 2014), we probed for the presence of several canonical EV markers, including ALIX, CD81, CD63, and TSG101. Vesicles isolated from both the control and TNF- $\alpha$ exposure groups expressed all of the expected markers (Figure 2C) indicating that EVs were indeed being isolated in the vesicle preparation.

\section{TNF- $\alpha$ alters miRNA expression intracellularly and in secreted EVs}

In order to determine the impact of acute TNF- $\alpha$ exposure on miRNA expression, we assayed miR-34a and miR-146a expression within cells and their secreted EVs 24-hours after exposure. Interestingly, intracellular expression levels of miR-34a were not changed after exposure to TNF- $\alpha$ (Figure 3A), yet a dose-dependent significant upregulation of this miRNA was observed in the secreted EVs (Figure 3B). Intracellular miR-146a expression was significantly increased after TNF- $\alpha$ exposure with an apparent ceiling effect (Figure 3C), and was significantly increased in a dose-dependent fashion in secreted EVs (Figure 3D).

\section{EVs are taken up by, and enhance mitochondrial function in recipient cells}

Because the secreted EVs contained TNF- $\alpha$ dose-dependent increased expression of both miR34a and miR-146a, we were interested in determining whether EV cargo could be delivered to TNF- $\alpha$ naïve recipient cells, and how this may affect their mitochondrial function. We exposed TNF- $\alpha$ naïve cells to EVs isolated from the media of TNF- $\alpha$ exposed cells whose RNA was fluorescently labeled; EVs delivered this RNA to naïve recipient cells (Figure 4). We then assessed mitochondrial function, using the $\mathrm{XF}^{\mathrm{e}} 96$ Mito Stress kit, in EV-recipient cells at 24 hours after exposure. To our surprise, we observed significant, dose-dependent increases in 
mitochondrial oxygen consumption after EV exposure (Figures 5A-D). Interestingly, we also observed significant increases in proton leak (Figure 5E) suggesting that although oxidative phosphorylation is increased, mitochondria are likely not functioning optimally, as protons are leaking across the membrane. We also observed a significant dose-dependent upregulation of basal glycolysis after exposure to EVs (Figure 5F). Unlike direct exposure to TNF- $\alpha$, only EVs induced from the highest concentration of TNF- $\alpha(10 \mathrm{ng} / \mathrm{ml})$ caused significant cell death in naïve cells (Figure 5G). The enhanced oxidative phosphorylation (Figures 5A-D) and glycolysis (Figure 5F) seen after exposure to TNF- $\alpha$-induced EVs is likely an underestimate of the bioenergetic function of the cells exposed to $10 \mathrm{ng} / \mathrm{ml} \mathrm{TNF}-\alpha-i n d u c e d \mathrm{EVs}$, as this group experienced significant cell death (Figure 5G).

\section{EVs increase ROS production in recipient cells}

Due to the increased proton leak observed in the Seahorse assay following EV exposure, we next assessed mitochondrial $\Psi \mathrm{m}$. We did not observe any significant changes in potential (Figure 6A) after a 24 hour exposure period to TNF- $\alpha$ induced EVs.

We next assessed ROS production in cells exposed to TNF- $\alpha$ induced EVs. We observed a statistically significant increase in mitochondrial superoxide production when cells were incubated with EVs induced by 0, 0.1, and $1 \mathrm{ng} / \mathrm{ml} \mathrm{TNF- \alpha}$, but not $10 \mathrm{ng} / \mathrm{ml}$ (Figure 6B); however this result may be attributed to the significant amount of cell death observed in this group (Figure 5G). Additionally, we observed significant increases in hydrogen peroxide $\left(\mathrm{H}_{2} \mathrm{O}_{2}\right)$ after exposure to EVs, with the highest amount generated in the $10 \mathrm{ng} / \mathrm{ml} \mathrm{TNF}-\alpha$ induced EV group (Figure 6C). As $\mathrm{H}_{2} \mathrm{O}_{2}$ has the capacity to induce neuronal cell death (Ricart and Fiszman, 2001), this may explain why we observed significant cellular death in this group (Figure 5G).

\subsection{Discussion}

AD is characterized by neuroinflammation (Wyss-Coray and Rogers, 2012) and mitochondrial dysfunction (Swerdlow, 2018). In the present study, we observed that physiologically relevant levels of the proinflammatory cytokine TNF- $\alpha$ are capable of causing significant cellular death 
and mitochondrial dysfunction in hippocampal neurons after a 24-hour exposure period. We also demonstrate that TNF- $\alpha$ induced EVs are taken up by naïve cells, and significantly alter mitochondrial activity in recipient cells. Specifically, we observed increases in mitochondrial respiration, glycolysis, proton leak, and ROS production in recipient cells after 24 hours of EV exposure. Together, these data suggest that direct exposure of TNF- $\alpha$ to a neuronal cell type is highly toxic, and the vesicles secreted in response to this exposure are capable of inducing a stress response in neighboring cells.

We observed significant downregulation of mitochondrial oxidative phosphorylation and glycolysis in HT-22 cells exposed to TNF- $\alpha$ for 24 hours. Under some conditions, when oxidative phosphorylation is inhibited or reduced, neurons can increase glycolysis as a protective, compensatory mechanism (Bas-Orth et al., 2017). However, with the decline in oxidative phosphorylation observed in this study, there is no compensatory increase in glycolysis. We additionally assessed expression of several electron transport chain proteins involved in oxidative phosphorylation (including NDUFC2 of complex I, SHDC of complex II, UQCRB of complex III, and Cytochrome C oxidase of complex IV) after TNF- $\alpha$ exposure, and observed no significant changes in expression (data not shown). High concentrations of LDH in the media, along with reductions in bioenergetic measurements indicate that these observations are likely a result of cell death due to exposure to the cytotoxic protein, TNF- $\alpha$.

We have previously shown that overexpression of miR-34a significantly reduces mitochondrial function in neuronal cells (Sarkar et al., 2016). In view of our evidence that miR-146a, but not miR-34a, was increased intracellularly in HT-22 cells, miR-146a may be responsible for the TNF- $\alpha$ induced mitochondrial impairment observed in these cells. Indeed, overexpression of miR-146a significantly reduces cellular bioenergetics (Jun et al., unpublished observation). Additionally, TNF- $\alpha$ exposure caused a dose-dependent increase in both miR-34a and miR-146a in secreted EVs. In line with other reports, these results suggest differential packaging and secretion mechanisms which are dependent on specific miRNAs (Abels and Breakefield, 2016). The results from the current study support the concept that some miRNAs (e.g. miR-34a) may be 
preferentially sorted out of cells (Jovičić and Gitler, 2017; Wei et al., 2017), however this interpretation is strictly speculative and requires further investigation.

As TNF- $\alpha$ exposure significantly elevated miR-34a and miR-146a expression within secreted EVs, we hypothesized that exposing naïve cells to TNF- $\alpha$ induced EVs would result in significant reduction of mitochondrial respiration due to miRNA-mediated repression of ETC protein translation. Interestingly, we observed significant increases in mitochondrial oxygen consumption in naïve cells exposed to these vesicles. In this study, only relative changes in miRNA expression were assessed, not definitive quantities. It has been postulated that there is fewer than 1 copy of an individual miRNA per EV (Chevillet et al., 2014; Wei et al., 2017), so it is likely that the absolute concentrations of miR-34a and miR-146a were not high enough to induce physiologically relevant repression of ETC proteins in recipient cells and cause reductions in oxygen consumption.

Several groups have also shown that exposure to EVs significantly increases mitochondrial respiration in recipient cells (Phinney et al., 2015; Sansone et al., 2017; Bland et al., 2018), and others have described that in response to stimulation, neurons are able to increase their rate of glycolysis (Yellen, 2018). This unexpected increase in mitochondrial activity may actually be a signal of mitochondrial stress, as we concomitantly observed dose-dependent increases in proton leak, suggesting that although oxidative phosphorylation is increased, the mitochondria are not actually functioning optimally. Indeed, a disproportionately large rate of mitochondrial oxygen consumption likely indicates an attempt to maintain $\Psi \mathrm{m}$ as proton leak across occurs (Jastroch et al., 2010). Spare respiratory capacity is an indicator of mitochondria's ability to respond to stress (Hill et al., 2009). Here we observe significant elevation of spare capacity in cells exposed to EVs, which is likely the mechanism by which mitochondria were able to maintain $\Psi$ m, despite undergoing significant proton leak. As mitochondria are the main generators of ROS, it is likely that the increased oxygen consumption incurred by maintaining $\Psi \mathrm{m}$ is responsible for significant ROS generation. Superoxide anions are quickly converted to less reactive $\mathrm{H}_{2} \mathrm{O}_{2}$ by superoxide dismutases (Lennicke et al., 2015), which may explain why we saw significantly more $\mathrm{H}_{2} \mathrm{O}_{2}$ than superoxide after EV exposure. 
Our observation of altered mitochondrial function after a single exposure to TNF- $\alpha$, or TNF- $\alpha$ induced EVs does not recapitulate the in vivo conditions of $\mathrm{AD}$, as there is constant crosstalk between different cell types, and persistent, dynamic EV-cell interactions. However, the current findings suggest that neuronal, inflammation-derived EVs may acutely alter mitochondrial activity in recipient cells. The mitochondrial cascade hypothesis of AD posits that increased ROS induces mitochondrial DNA damage and reduces mitochondrial function; with time this initiates a "reset response" in which $A \beta$ production is increased, leading to further generation of ROS in a vicious, positive feedback cycle (Swerdlow and Khan, 2004). While we have not identified the cargo within EVs that initiates this mitochondrial response, future studies will address this issue, as any component of EV-associated cargo may be responsible for these effects, e.g. direct transfer of specific proteins, mRNAs, or other miRNAs not assessed here.

Importantly, the current study provides novel insights on the role of inflammation-induced EVs in the inflamed and aging brain. The results underscore the importance of TNF- $\alpha$, a canonical proinflammatory cytokine in the brain, as a regulator of mitochondrial function, miRNA expression, and EV secretion in neurons. Thus, TNF- $\alpha$ induced EVs may be drivers of the bioenergetic function and dysfunction throughout the brain in AD and other neuroinflammatory disorders. 


\section{Figure Legends}

Figure 3.1: TNF- $\alpha$ exposure significantly reduces cellular bioenergetic function and increases cell death. After 24 hours exposure to TNF- $\alpha$, basal respiration $(A ; F 3,292=11.90, p<0.001)$, maximal respiration $(\mathrm{B} ; \mathrm{F} 3,292=42.31, \mathrm{p}<0.0001)$, ATP production $(\mathrm{C} ; \mathrm{F} 3,292=39.80, \mathrm{p}<$ $0.0001)$, and spare capacity $(\mathrm{D} ; \mathrm{F} 3,292=50.10, \mathrm{p}<0.0001)$ were significantly reduced at the highest two concentrations. Proton leak $(E ; F 3,202=10.62$, p < 0.0001) was significantly increased. Glycolysis was also significantly reduced after TNF- $\alpha$ exposure $(F ; F 3,164=59.42$, p $<0.0001)$. LDH data indicate that significant cell death occurred at all concentrations of TNF- $\alpha$ exposure $(\mathrm{G} ; \mathrm{F} 3,223=65.31, \mathrm{p}<0.0001) .{ }^{*} \mathrm{p}<0.05, * * \mathrm{p}<0.01, * * * \mathrm{p}<0.001$, and $* * * * \mathrm{p}<$ 0.0001 .

Figure 3.2: TNF- $\alpha$ exposure induces secretion of EVs. Particle size distributions calculated from the NanoSight NS300 show expected size ranges for EVs (A; F3,3996 = 7.06, p < 0.0001). Overall particle concentrations for each TNF- $\alpha$ exposure group indicate that TNF- $\alpha$ exposure induces vesicle secretion (B). TNF- $\alpha$ induces EVs expressing canonical markers and are appropriately sized. Canonical EV proteins and their locations on a dot blot are shown (C). Presence of proteins expressed for control vesicles (C, top panel) and TNF- $\alpha(10 \mathrm{ng} / \mathrm{ml})$ induced vesicles $(\mathrm{C}$, bottom panel) are shown. $* \mathrm{p}<0.05, * * * * \mathrm{p}<0.0001$.

Figure 3.3: TNF- $\alpha$ significantly alters miRNA expression profiles. TNF- $\alpha$ exposure did not significantly alter intracellular expression of miR-34a $(\mathrm{A} ; \mathrm{F} 3,32=1.29, \mathrm{p}=0.2951)$, but did induce a dose-dependent increase in miR-34a expression within EVs (B; F3,31 = 30.00, p < 0.0001). Conversely, TNF- $\alpha$ exposure induced intracellular upregulation of miR-146a which trended towards significance $(\mathrm{C} ; \mathrm{F} 3,32=2.92, \mathrm{p}=0.0643)$, as well as a significant, dosedependent increases within EVs (D; F3,31 = 13.15, p < 0.0001). *p < 0.05, **p < 0.01, ***p < 0.001 , and $* * * * \mathrm{p}<0.0001$.

Figure 3.4: Naïve cell EV uptake and functional consequences. EVs derived from control and all concentrations of TNF- $\alpha(0,0.1,1$, and $10 \mathrm{ng} / \mathrm{ml})$ are taken up by naïve cells as evidenced by punctate staining of red-labeled EV RNA present within naïve cells. Scale bar $=40 \mu \mathrm{m}$. 
Figure 3.5: TNF- $\alpha$ induced EVs (at all concentrations) significantly increased basal respiration $(\mathrm{A} ; \mathrm{F} 4,67=6.72, \mathrm{p}=0.0001)$ and maximal respiration $(\mathrm{B} ; \mathrm{F} 4,67=15.05, \mathrm{p}<0.0001)$, while all vesicles significantly increased ATP production $(C ; F 4,67=3.64, p=0.0096)$ and spare capacity (D; F4,67 = 21.50, p < 0.0001). Proton leak was also significantly increased at the highest TNF- $\alpha$ concentrations $(\mathrm{E} ; \mathrm{F} 4,67=3.07, \mathrm{p}=0.0221)$. Exposure to these vesicles significantly increased glycolysis as well (F; F4, $84=5.024, \mathrm{p}<0.0011)$. Significant cell death only occurred at the highest concentration $(\mathrm{G} ; \mathrm{F} 4,44=4.717, \mathrm{p}=0.003)$. ${ }^{*} \mathrm{p}<0.05, * * \mathrm{p}<0.01$, and $* * * \mathrm{p}<0.001$.

Figure 3.6: TNF- $\alpha$ induced EVs alter ROS production in recipient cells. EVs do not have a significant impact on the inner mitochondrial membrane potential (A; F4,115 $=1.287, \mathrm{p}=$ 0.2794). Exposure of naïve cells to EVs significantly increases ROS production in the form of mitochondrial superoxide $(\mathrm{B} ; \mathrm{F} 4,113=2.787, \mathrm{p}=0.0298)$, and hydrogen peroxide $(\mathrm{C} ; \mathrm{F} 4,85=$ 5.329, $\mathrm{p}=0.0007)$. 
A

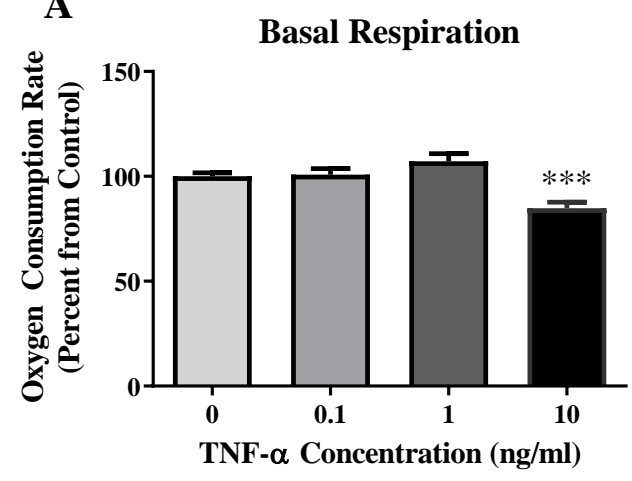

C

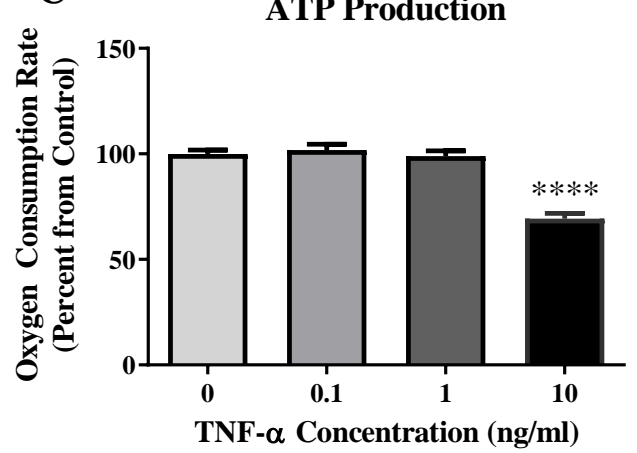

E

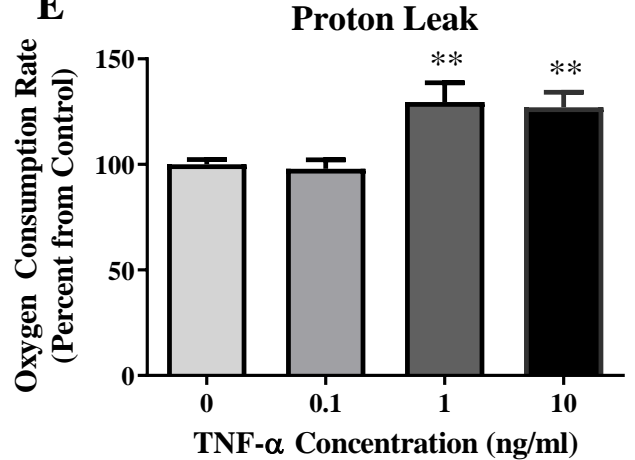

G LDH

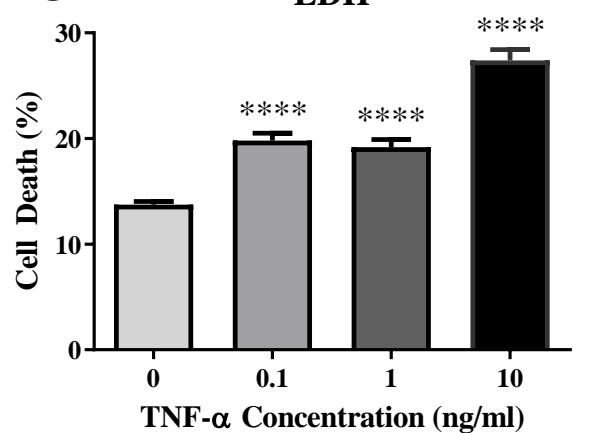

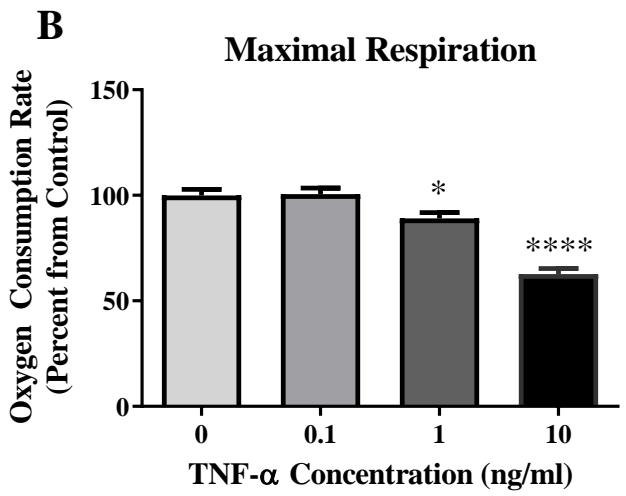
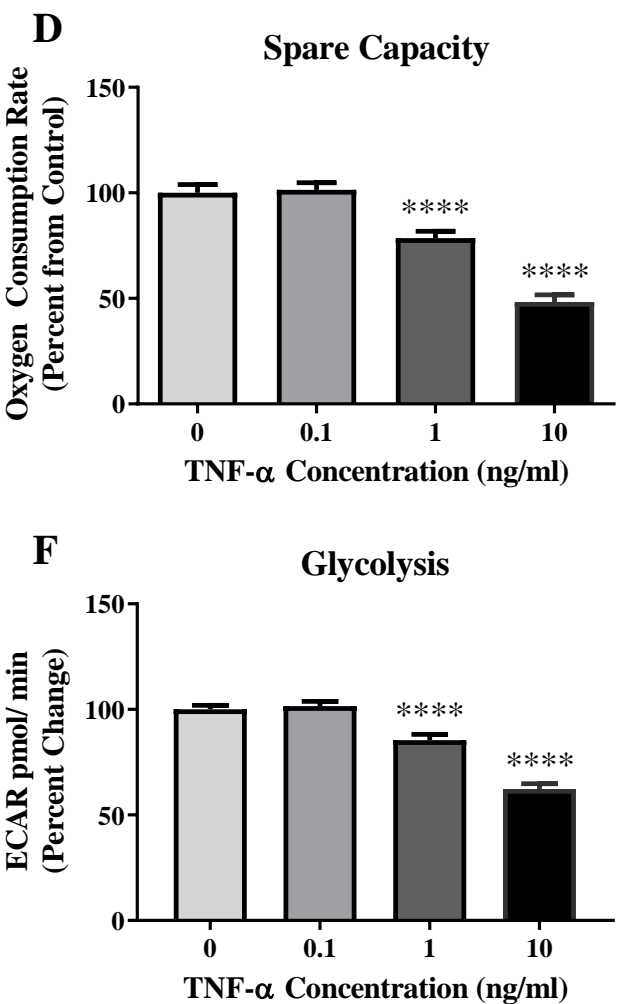
Figure 3.1
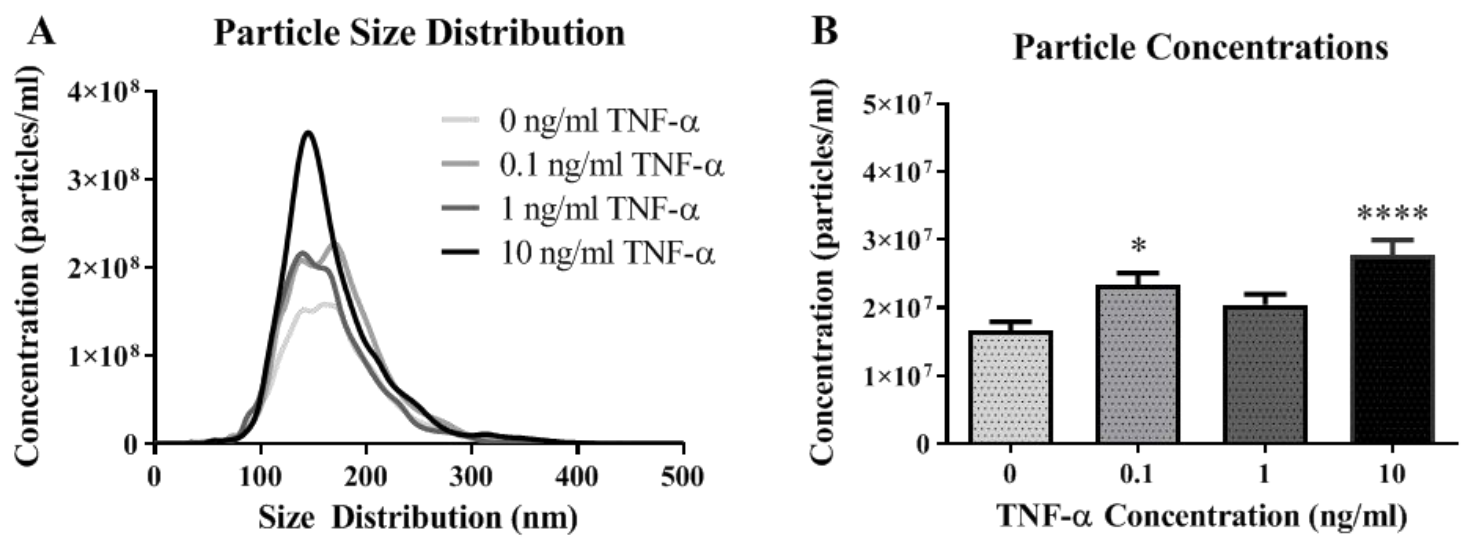

C
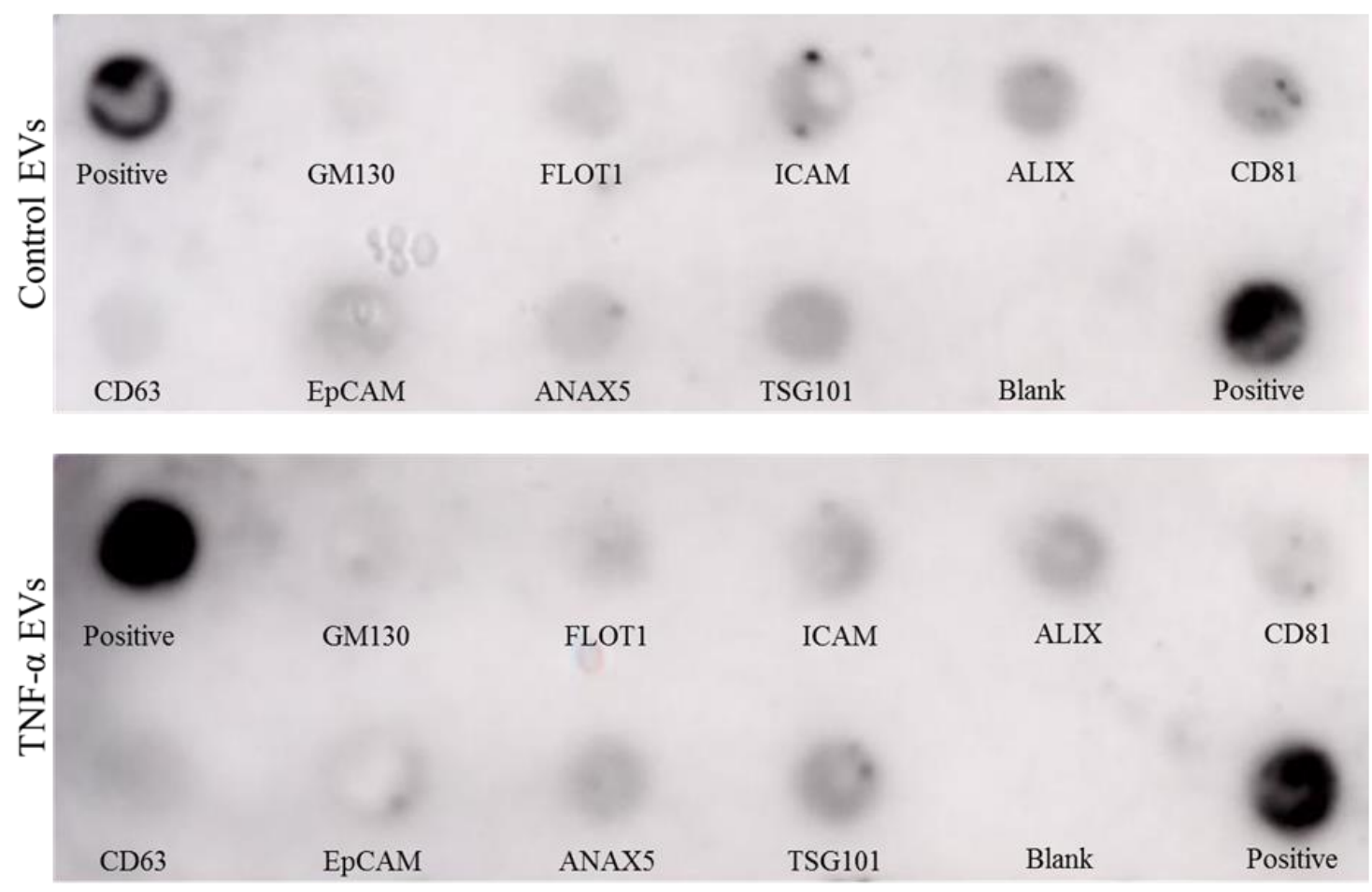

Figure 3.2 

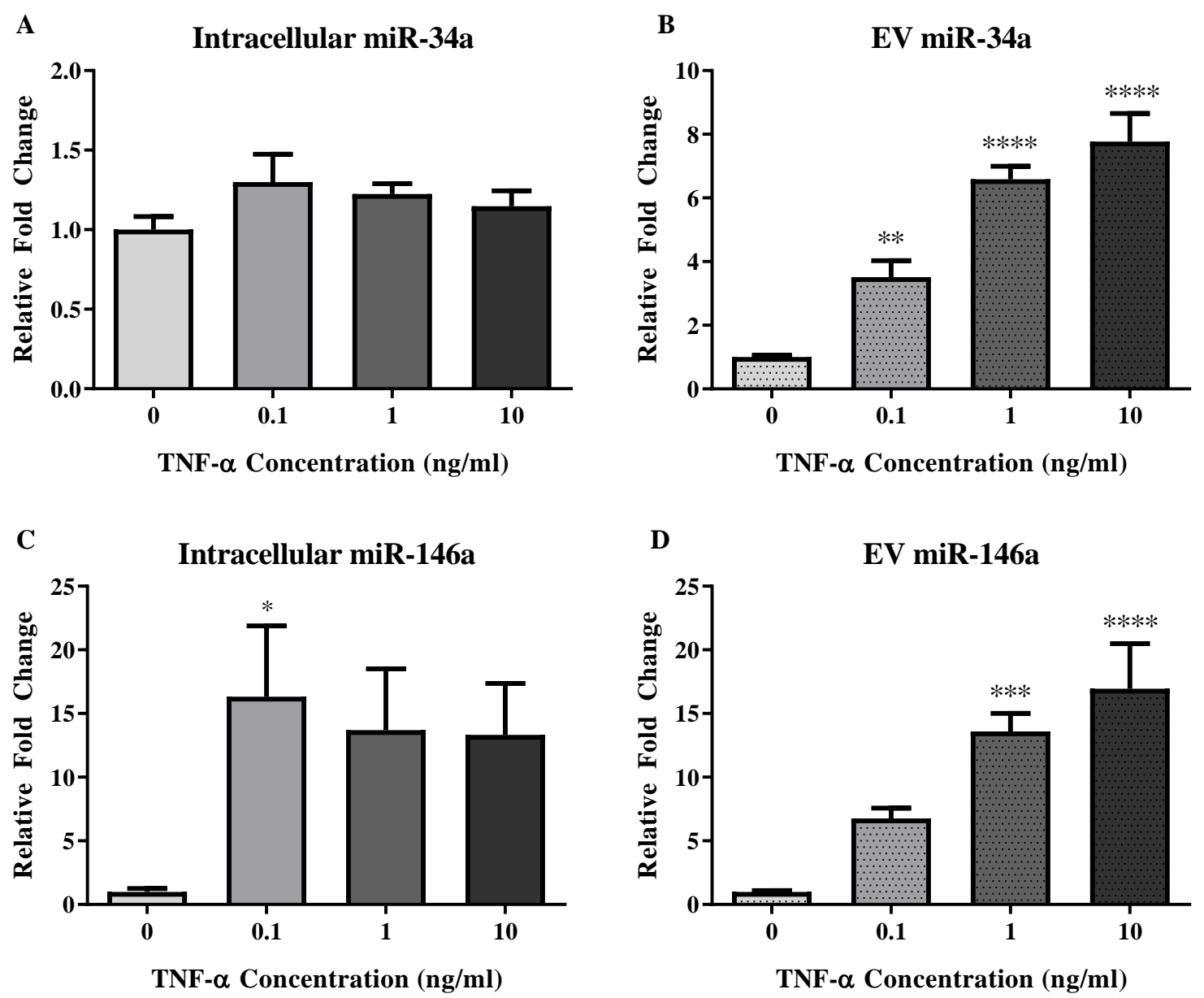

Figure 3.3 


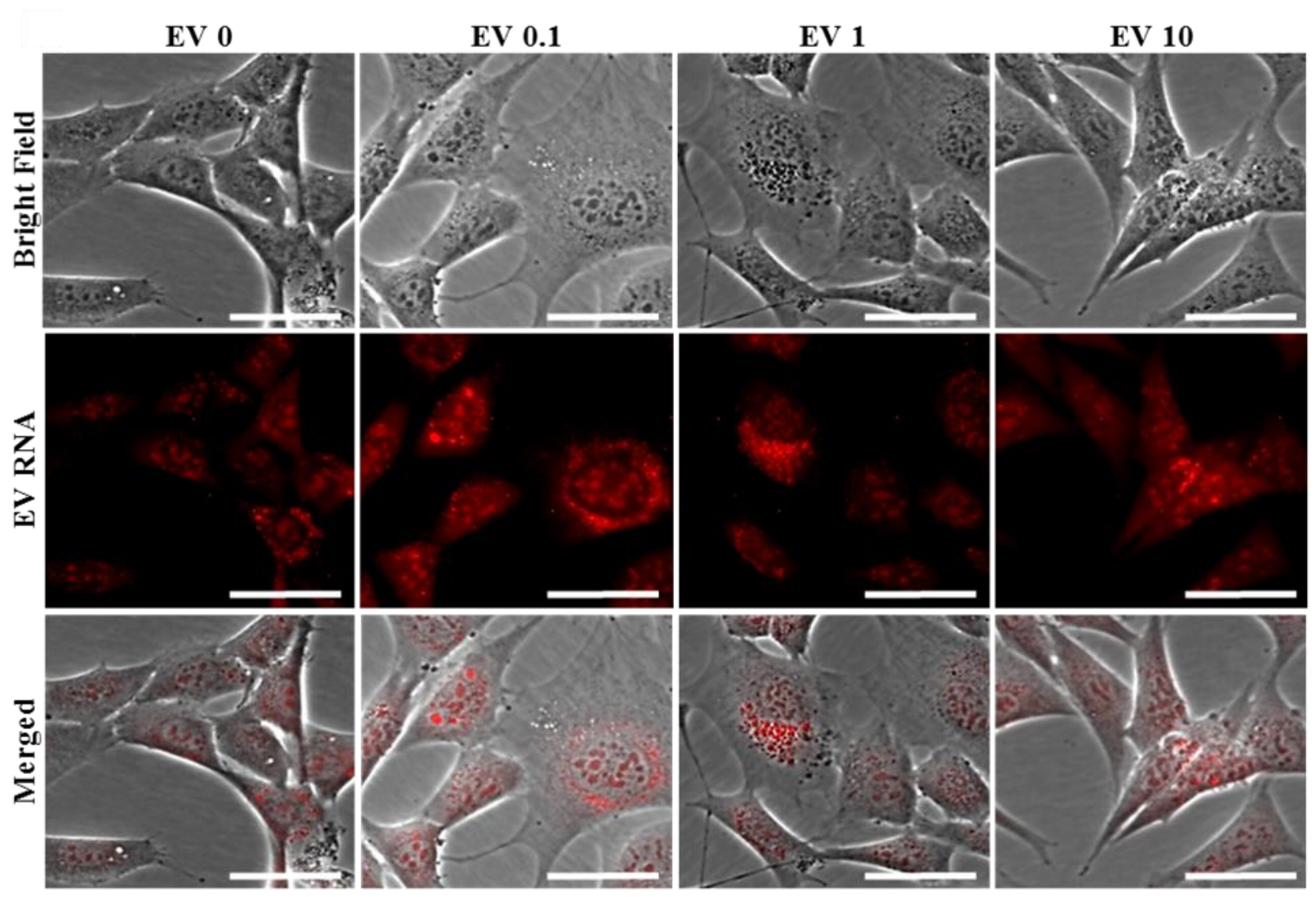

Figure 3.4 


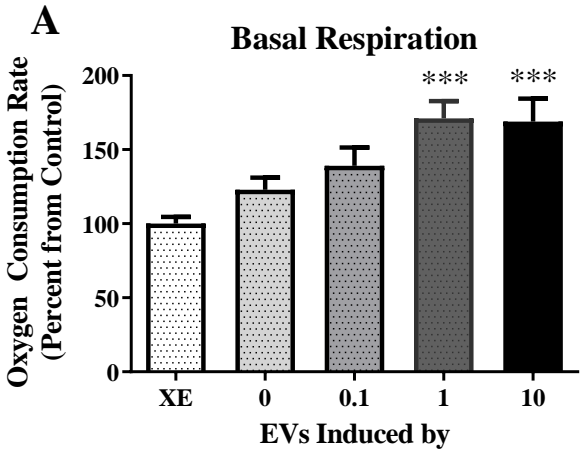

TNF- $\alpha$ Concentration (ng/ml)

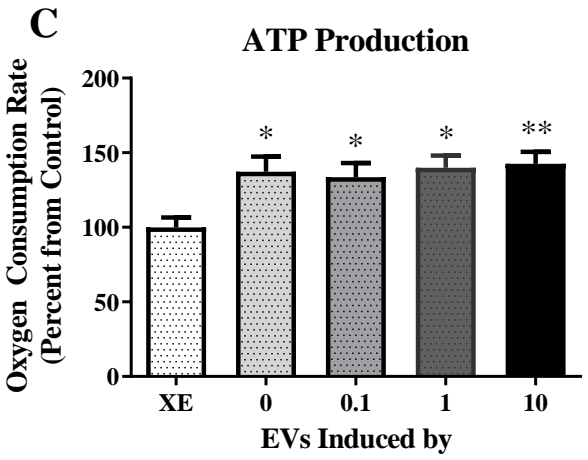

TNF- $\alpha$ Concentration (ng/ml)

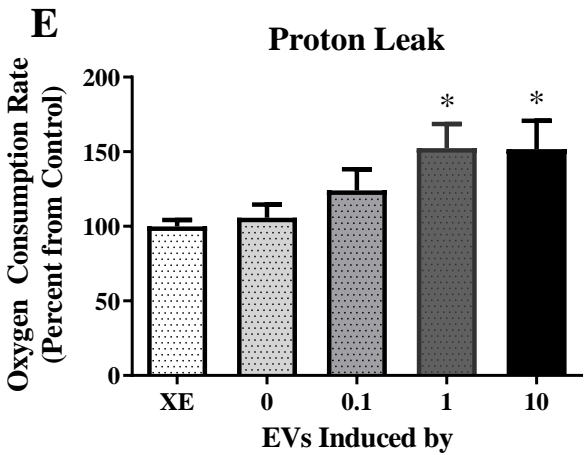

TNF- $\alpha$ Concentration (ng/ml)

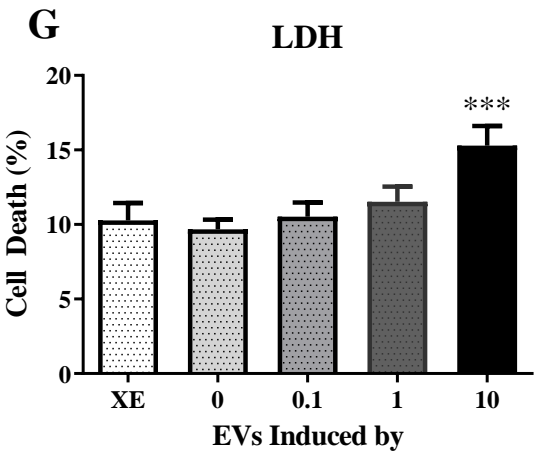

TNF- $\alpha$ Concentration (ng/ml)

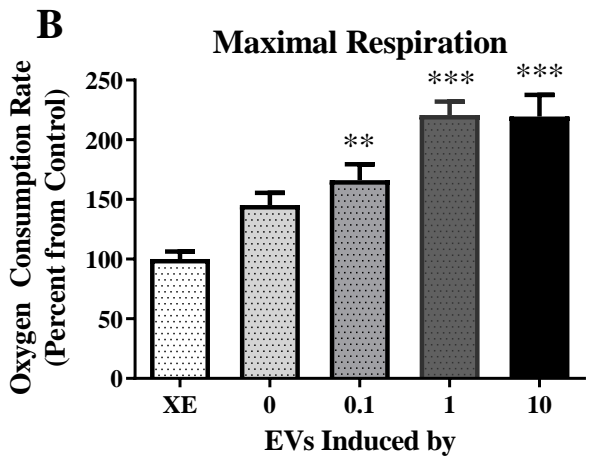

TNF- $\alpha$ Concentration (ng/ml)
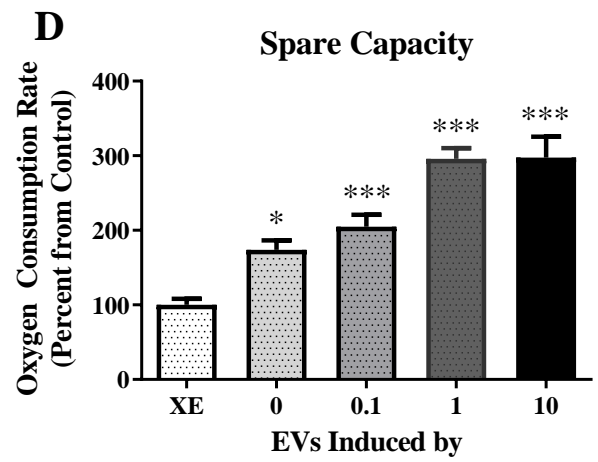

TNF- $\alpha$ Concentration (ng/ml)
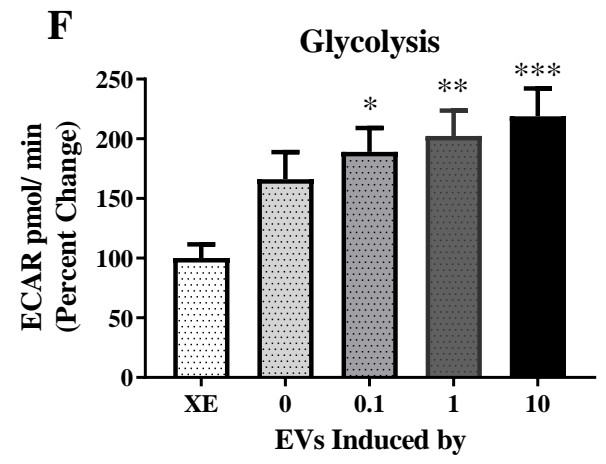

TNF- $\alpha$ Concentration (ng/ml) 
Figure 3.5 

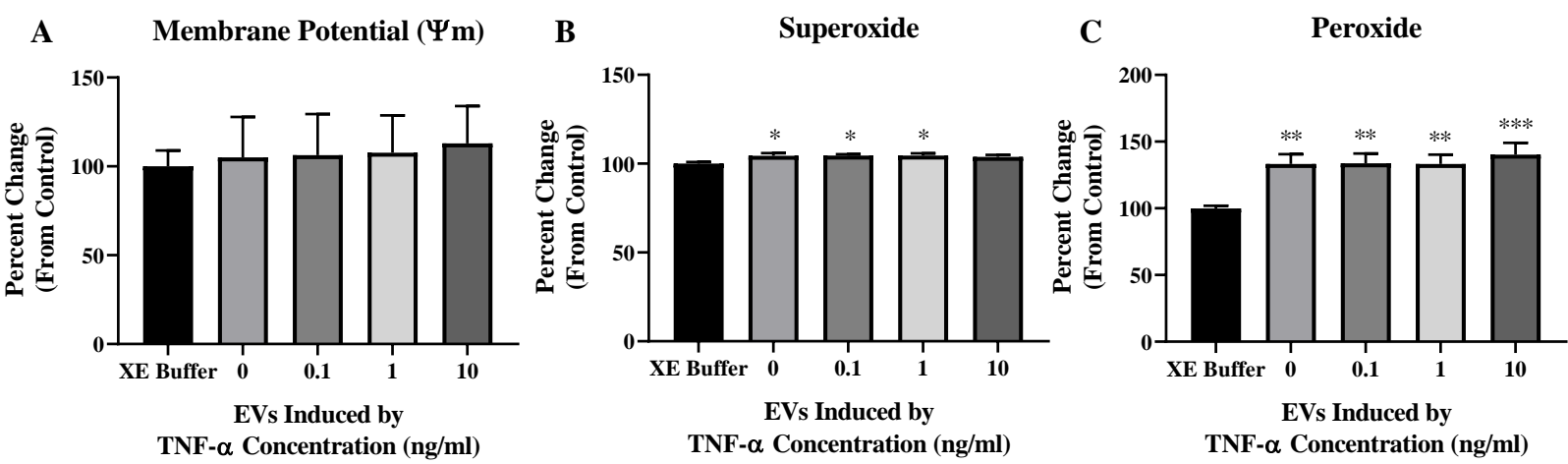

Figure 3.6 


\section{Chapter 4}

\section{Intermittent lipopolysaccharide exposure significantly increases cortical infarct size}

\section{Ashley E. Russell, John Z. Cavendish, Duaa Dakhlallah, Heung Hu, Xeufang Ren, and James W. Simpkins}

Author Contribution: AR, JC, DD, XR, and JS designed studies; AR, JC, HH, and XR performed research; AR analyzed data; AR and JS wrote the paper.

Russell AE, Cavendish JZ, Dakhlallah D, Hu H, Ren X, Simpkins JW (2019) Intermittent lipopolysaccharide exposure significantly increases cortical infarct size. In progress. 


\subsection{Abstract}

Stroke is a leading cause of death and disability in the United States. Traditional risk factors like hypertension, diabetes, and obesity do not fully account for all stroke cases. Recent infection is also now regarded as a stroke risk factor, as changes in systemic immune signaling can increase risk of thrombosis formation. We have previously shown that administration of lipopolysaccharide (LPS) 30 minutes prior to stroke induces significant increases in infarct volume. In the current study, we assessed the effects of intermittent LPS exposure, with a long recovery period, on stroke infarct volume. We found that animals repeatedly exposed to LPS have significantly larger cortical infarcts when compared to saline controls. To elucidate the mechanism behind this phenomenon, several avenues were investigated. We found significant upregulation of TNF- $\alpha$ mRNA, especially in the ipsilateral hemisphere of both saline and LPS exposed groups when compared to sham surgery animals. Further, we assessed expression of genes involved in autophagy (Beclin-1, ATG5, LC3a, and LC3b) and observed significant reduction in their expression in the ipsilateral hemisphere of the LPS stroke animals. Transcription of several of these genes may be regulated by DNA methylation, which is mediated by DNA methyltransferases (DNMTs). We assessed mRNA expression of DNMT1, DNMT3a, and DNMT3b and observed a significant downregulation of DNMT1 and DNMT3a in the ipsilateral hemisphere of LPS stroke animals, suggesting that changes in DNA methylation occur in response to stroke after repeated LPS exposure. 


\subsection{Introduction}

Stroke is the fifth leading cause of death and a major cause of disability in the United States (Yang et al., 2017). Traditionally recognized risk factors for stroke and other acute vascular events include hypertension, diabetes, obesity, and tobacco use (Yang et al., 2017). In addition, recent (7-10 days) bacterial or viral infections are also recognized as potential stroke risk factors (Grau et al., 1995, 2005; Bova et al., 1996; Paganini-Hill et al., 2003). Systemic infection can alter thrombosis formation by increasing platelet aggregation (Zeller et al., 2005; Elkind et al., 2011) and increase circulating cytokine concentrations (Wilson et al., 1998; Guedes et al., 2016) which have been shown to play a major role in fibronectin accumulation (Pawluczyk and Harris, 1998) and thereby contribute to thrombosis formation (Maurer et al., 2010). Additionally, systemic inflammation can induce changes in endothelial cell function (Stern et al., 1988) which may contribute to the development of atherosclerosis (Sessa et al., 2014; Campbell and Rosenfeld, 2015).

Previous work from our laboratory has found that exposure to lipopolysaccharide (LPS) 30 minutes prior to experimental stroke significantly increases infarct volumes when compared to vehicle injected controls (Doll et al., 2015b). In the current study, we sought to expand these findings and determine if intermittent LPS exposure could be additive over time, increasing infection burden of the organism, and lead to significantly larger stroke infarcts. In an effort to mimic the rate of illness experienced by an adult human, we induced an immune response in mice every two weeks (akin to $\sim 2$ years in a human), with ample time to fully recover between insults. Despite animals being fully recovered from the last LPS injection at the time of surgery, we observed a significant increase in infarct size in the LPS treated group when compared to the saline controls. We further explored this finding by assessing changes in gene expression within the ipsilateral and contralateral hemispheres of the brains.

\subsection{Materials and Methods}

Animals 
Twenty four male C57BL/6J mice (12 weeks of age) were acquired from Jackson Laboratory (Bar Harbor, ME). Animals were housed in sterile micro-isolator cages with sterile bedding, and provided food and water ad libitum in the West Virginia University Health Science Center animal facility. All procedures were conducted according to criteria approved by the Institutional Animal Care and Use Committees at West Virginia University.

\section{Intermittent immune activation paradigm}

Lipopolysaccharide (LPS; Escherichia coli 055:B5, Sigma, St. Louis, MO) was reconstituted in sterile, injectable saline (B. Braun Medical Inc, Irvine, CA). Animals were administered LPS (or an equal volume of saline) via intraperitoneal (i.p.) injection. To ensure that animals elicited an immune response, the concentration of LPS was doubled with each subsequent injection. Each injection was separated by a two-week recovery period to allow for animals' health to return to baseline levels (Figure 1).

\section{Sickness behavior monitoring}

To measure animals' sickness behavior prior to, and after each LPS (or saline) injection, a 0-20 point scale of overall health was implemented (Doll et al., 2015a). For the majority of the health screen, animals were observed in their home cage to assess behavior across several parameters, including general appearance, posture, respiration, and spontaneous locomotion/social interaction. Animals were removed from the cage to then assess their body condition, temperature, and weight. A score was assigned for each parameter, which were then summed to provide an overall assessment of the animal's health.

Prior to the beginning of the injection paradigm, baseline health screen measurements were obtained, which were then used for determining changes from the norm for each individual animal. Four hours post-injection, the health screen was administered to ensure animals were exhibiting a sickness response due to LPS exposure. Additionally, 13 days post-injection the health screen was conducted to ensure animals recovered to baseline levels of health (Figure 1).

Transient middle cerebral artery occlusion and sham surgery 
Surgical anesthesia was induced with 4-5\% isoflurane and maintained with 1-2\% isoflurane via face-mask in oxygen-enriched air. Transient middle cerebral artery occlusion (tMCAO) model was performed with a 6.0 monofilament suture (Doccol, Sharon, Massachusetts) for seventy minutes, with rectal body temperature maintained at $37^{\circ} \mathrm{C} \pm 0.5^{\circ} \mathrm{C}$ during surgery with a warm blanket (Stoelting Co, Wood Dale, IL). To confirm successful occlusion (>70\% decrease in blood flow), regional cerebral blood flow was detected using laser Doppler flowmetry (Moor instruments, United Kingdom). All surgeries were performed by one surgeon who was blinded to pre-treatments.

\section{Exclusion criteria for animal experiments}

Several exclusion criteria for tMCAO were implemented in this experiment, as we have previously described (Doll et al., 2015b; Sun et al., 2016). (1) A decrease of regional cerebral blood flow < 70\% during occlusion as detected by laser Doppler flowmetry; (2) surgery time lasting > 70 minutes; (3) neurological score 0 , indicating no neurologic deficit 3 hours after tMCAO; (4) no infarction in the MCA territory as indicated by TTC staining; (5) subarachnoid hemorrhage upon post-mortem examination; (6) substantial ambient temperature changes in the animal housing facility; and (7) body temperature at or below $32^{\circ} \mathrm{C}$.

In this study, 3 mice were excluded: 1 mouse (saline treated) because the laser Doppler flowmetry did not reach 70\% reduction during the occlusion, and 2 mice (LPS treated) because body temperatures were below $32^{\circ} \mathrm{C}$.

\section{Analysis of cerebral infarct volume}

All animals were euthanized 24 hours post-ischemia via cardiac puncture. Animals were then perfused with 1X phosphate buffered saline (PBS). Brain from 3 saline and 3 LPS animals were submerged in formaldehyde for later histological confirmation of infarct (data not shown). The remaining brains were removed and cut into $2-\mathrm{mm}$ sections with a mouse brain matrix. Sections were stained with 2\% 2,3,5-triphenyltetrazolium chloride (TTC; Sigma, Saint Louis, Missouri) in PBS solution at $37^{\circ} \mathrm{C}$ for 15 minutes, and digitally photographed. 
After imaging TTC stained brain slices, brain tissue was dissected into four groups. The main infarcted tissue was dissected away from each of the slices and pooled together as the ipsilateral core, while the remaining tissue from the ipsilateral hemisphere was pooled together as the ipsilateral penumbra. Mirroring sections were taken from the contralateral hemisphere and termed contralateral core and contralateral penumbra. Tissue was snap frozen in liquid nitrogen and stored at $-80^{\circ} \mathrm{C}$. Each pooled sample was homogenized for later RNA analysis as an independent sample.

Digitized images of each brain section were analyzed using computerized image analysis software (ImagJ, NIH) in a blinded manner. Brain and infarct volumes for right (ipsilateral) and left (contralateral) hemispheres were calculated. Volumes were calculated as a percentage of contralateral cortex, striatum, and total hemisphere to reduce the effect of brain edema.

\section{RNA isolation and PCR}

Total RNA, including miRNAs, was isolated from brain tissue via Trizol extraction (Invitrogen). $500 \mu \mathrm{l}$ Trizol was added to each sample and vortexed; $125 \mu 1$ chloroform was next added to the sample and vortexed. After a 10 minute incubation period on ice, samples were centrifuged at $15,000 \mathrm{rpm}$ for 15 minutes at $4^{\circ} \mathrm{C}$. The upper aqueous layer was transferred to a new microfuge tube, followed by the addition of $500 \mu \mathrm{l}$ isopropanol and $1 \mu \mathrm{l}$ glycogen. The samples were stored at $-20^{\circ} \mathrm{C}$ for $24-48$ hours to allow for RNA precipitation. The samples were centrifuged for 10 minutes at 15,000 rpm and washed twice with $200 \mu \mathrm{l}$ of $75 \%$ ethanol. RNA was dried and resuspended in $20 \mu 1$ RNase free water.

RNA concentrations were measured with a NanoDrop One Microvolume UV-Vis Spectrophotometer (ThermoFisher), and $1 \mu \mathrm{g}$ was reverse transcribed using the SuperScriptTM III First-Strand Synthesis SuperMix for qRT-PCR (Invitrogen) as per the manufacturer's instructions. Briefly, $10 \mu \mathrm{l}$ 2X RT Reaction Mix, $2 \mu \mathrm{l}$ RT Enzyme Mix, and $8 \mu 1 \mathrm{cDNA}$ were incubated at $25^{\circ} \mathrm{C}$ for 10 minutes, $50^{\circ} \mathrm{C}$ for 30 minutes, and $85^{\circ} \mathrm{C}$ for 5 minutes. $1 \mu \mathrm{E}$. coli RNase $\mathrm{H}$ was added to each sample, then incubated at $37^{\circ} \mathrm{C}$ for 20 minutes. 
Additionally, $1 \mu \mathrm{g}$ total RNA containing microRNAs was reverse transcribed using the miScript II RT kit (Qiagen) as per the manufacturer's instructions. Briefly, $2 \mu 1$ 5x miScript HiSpec Buffer, $1 \mu 1$ 10x miScript Nucleics Mix, $1 \mu 1$ miScript Reverse Transcriptase Mix, and $6 \mu 1$ Template RNA/nuclease-free water were incubated at $37^{\circ} \mathrm{C}$ for 60 minutes and $95^{\circ} \mathrm{C}$ for 5 minutes. Predesigned PrimeTime qPCR primers for RPLA and CAP-1 (internal controls), as well as TNF- $\alpha$, Beclin-1, ATG5, LC3a, LC3b, DNMT1, DNMT3a, and DNMT3b were purchased from Integrated DNA Technologies (Coralville, IA).

Expression of miR-155, -181a, and -181c was assayed using target specific miScript primer assays and the miScript SYBR ${ }^{\circledR}$ Green PCR kit (Qiagen) (5 $\mu 1$ SYBR ${ }^{\circledR}$ Green; $1 \mu 1$ Universal Primer; $1 \mu$ Target Primer; $3 \mu$ diluted cDNA) using the CFX384 Touch ${ }^{\text {TM }}$ Real-Time PCR Detection System (Bio-Rad, Hercules, CA, USA) for 45 cycles as follows: $15 \mathrm{sec}$ at $94{ }^{\circ} \mathrm{C}, 30$ sec at $55^{\circ} \mathrm{C}, 30 \mathrm{sec}$ at $70{ }^{\circ} \mathrm{C}$. All miRNA specific primers were miScript Primer Assays (Qiagen); Hs_RNU6-2_11 (MS00033740), and Hs_miR-155 (MS000031486); Hs_miR-181a (MS00008827); and Hs_miR-181c (MS00008841). The expression levels of target genes were standardized against RNU6.

Quantification of PCR amplified mRNA and microRNA specific cDNA was done by comparative cycle threshold CT method (2- $\Delta \Delta \mathrm{CT})$. Two independent values were obtained for each hemisphere (core and penumbra) for all animals. Fold changes of target gene expression in the ipsilateral and contralateral hemispheres of saline or LPS exposed stroke animals was relative to that of the corresponding hemisphere of the sham animals. cDNA could not be synthesized from samples with low RNA yield (<1ug total RNA), and were excluded from analysis. Significant outliers, as determined by GraphPad Prism 8.0, were excluded from analysis.

\section{Statistical analyses}

All quantitative data were assessed for significance using either a one-way ANOVA with Dunnett's post hoc test, or Student's t test. All results were analyzed by GraphPad Prism 8.0 software (GraphPad Software, La Jolla, CA). Results from the experiments are reported as means 
\pm SEM. A p value $<0.05$ was used to establish significance; significance presented as ${ }^{*} \mathrm{p}<0.05$ $* * \mathrm{p}<0.01, * * * \mathrm{p}<0.001$, and $* * * * \mathrm{p}<0.0001$.

\subsection{Results}

LPS injections significantly alter animal health status

LPS exposure induces an acute sickness response, from which animals recovered (Figure 2). Throughout the duration of the experiment, there were no significant changes in health screen scores for animals exposed to saline; thus all health screen data for these animals was grouped together. Animals exposed to LPS exhibited significant sickness behaviors four hours postinjection after injections 1, 2, and 4, while injection 5 trended towards significance. At 13 days post-LPS, health screen scores averaged $<1$, indicating that animals returned to normal health status between injections.

Repeated LPS exposure significantly increases cortical infarct volume

By day 13-post injection 5, all animals exhibited a full behavioral recovery. Despite this, we observed a significant increase in stroke infarct volume in animals that were intermittently injected with LPS, compared to the saline-injected controls (Figure 3). There were significantly larger infarct volumes in the cortex and total ipsilateral hemisphere in the LPS treated animals compared to saline; however, there were no significant differences in striatal infarct volume between the two groups (Figure 3B).

\section{TNF- $\alpha$ mRNA transcription is significantly upregulated 24 hours post-stroke}

Due to our findings that intermittent exposure to LPS significantly increased infarct size, we sought to better understand the mechanism by which this effect was occurring. Tumor necrosis factor-alpha (TNF- $\alpha$ ) is a proinflammatory cytokine which is thought to play an important role in the post-stroke immune response (Barone et al., 1997). We measured TNF- $\alpha$ mRNA in the contralateral and ipsilateral hemispheres of saline or LPS-injected animals that underwent tMCAO, as well as sham surgery controls (Figure 4). We found a significant increase in TNF- $\alpha$ mRNA expression in both hemispheres of the animals subjected to tMCAO when compared to 
the sham surgery group. Interestingly, TNF- $\alpha$ mRNA expression increased in both saline and LPS groups $\sim 5$-fold in the contralateral hemisphere (Figure 4A), while in the ipsilateral hemisphere, TNF- $\alpha$ mRNA was increased $\sim 50$-fold in the saline group, but only $\sim 35$-fold in the LPS group (Figure 4B).

\section{LPS exposure significantly alters post-stroke autophagy-associated mRNAs}

As changes in TNF- $\alpha$ mRNA expression did not appear to be the driving force leading to increased infarct size after repeated LPS exposure, we examined expression of various genes associated with autophagy. As shown in Figure 5, we observed significant reductions in all four autophagy genes in the ipsilateral hemispheres of at 24-hours post-stroke in the LPS exposed animals when compared to shams (Figure 5B, D, F, H). Beclin-1 was also significantly reduced in both hemispheres of the saline exposed stroke animals when compared to sham (Figure 5A and $\mathrm{B})$.

\section{Stroke alters miRNA profiles}

Post-stroke alterations in gene expression, including that of microRNAs (miRNAs), has been observed by many groups, (as reviewed by Sarkar et al., 2018). In the ipsilateral hemisphere, we observed significant upregulation of miR-155 (Figure 5B) in both the saline and LPS treated stroke animals, when compared to sham surgery controls. Significant downregulation of miR181a (Figure 5D) was observed in both stroke groups, while significant downregulation of miR181c (Figure 5F) was only observed in the LPS stroke group.

\section{LPS exposure prior to stroke significantly reduces DNMT mRNA expression}

De novo DNA methylation occurs via DNMT3a and DNMT3b, while DNMT1 functions to maintain methylation (Okano et al., 1999). We assessed changes mRNA expression of these DNMTs to indirectly assess the possibility of inflammation-induced changes in DNA methylation. We observed a significant downregulation of DNMT1 mRNA expression in both the contralateral (Figure 7A) and ipsilateral (Figure 7B) hemispheres of stroke animals exposed to LPS when compared to sham controls. We also observed significant downregulation of DNMT3a mRNA expression in the contralateral hemisphere (Figure 7C) of LPS exposed stroke 
animals, and downregulation in the ipsilateral hemispheres (Figure 7D) of both saline and LPS exposed stroke animals. We observed no significant changes in DNMT3b expression (Figures 7E and F).

\subsection{Discussion}

In this study, we found that intermittent immune activation with the gram-negative bacterial wall component, LPS, induces significant sickness behaviors 4 hours post-injection, and that animals recover from this effect in < 13 days. Despite making a full recovery after the last injection of LPS, animals subjected to tMCAO had significantly larger infarct volumes at 24-hours reperfusion when compared to saline injected animals. We investigated several avenues to elucidate the mechanism behind this phenomenon. Consistent with the literature, we found significant upregulation of TNF- $\alpha$ mRNA in the brains of stroke animals, when compared to sham controls. We also assessed expression of autophagy related mRNAs and found significant reductions in expression within the ipsilateral hemispheres of the LPS exposed stroke mice. Reduced autophagic function can lead to the accumulation of damaged proteins and organelles and lead to cellular apoptosis and necrosis. We next assessed changes in miRNAs previously implicated in stroke which may be involved in reducing autophagy gene expression, as well as DNMTs that may be altering the methylation status of the autophagy genes.

As infarct size is significantly increased after exposure to LPS, we sought to determine the mechanism by which this occurs. At the transcriptional level, TNF- $\alpha$ mRNA is significantly upregulated in post-stroke brains (Pan and Kastin, 2007), and protein expression in blood plasma of stroke patients 24 hours post-insult has been positively correlated with infarct volume (Zaremba and Losy, 2001). Administering exogenous TNF- $\alpha$ prior to experimental stroke significantly exacerbates infarct volume, while blocking endogenous TNF- $\alpha$ signaling poststroke has shown to be neuroprotective (Barone et al., 1997). Previous work has also demonstrated that systemic administration of LPS can induce TNF- $\alpha$ mRNA expression (Buttini et al., 1997). Further, it is well established that systemic inflammation activates the sympathetic nervous system (Pongratz and Straub, 2014), and can lead to enhanced transcription of TNF- $\alpha$ 
mRNA within the brain (Zielinski et al., 2013). We hypothesized that our observation of LPSinduced increases in stroke infarct volume may be mediated by increased expression of TNF- $\alpha$ 24 hours post-stroke. Despite observing significant post-stroke upregulation of TNF- $\alpha$ mRNA expression, we observed no significant differences in TNF- $\alpha$ mRNA expression between saline and LPS exposed stroke animals, indicating that another mechanism is likely underlying this phenomenon.

Although not significant, the saline exposed stroke animals expressed higher levels of TNF- $\alpha$ mRNA ( 50-fold) than the LPS exposed stroke animals ( 35-fold). A recent study described an interesting LPS-mediated effect of immune tolerance within the brain. Two consecutive injections of LPS were required to initiate an immune response within the brain, however this effect was attenuated by the fourth injection (Wendeln et al., 2018). We may have observed reduced TNF- $\alpha$ mRNA in the LPS group, compared to the saline, due to immune tolerance, as these animals already had several experiences with brain-immune activation, while the animals injected with saline were experiencing a significant inflammatory event for the first time (i.e. the stroke). We also observed attenuation of sickness behavior with each subsequent LPS exposure, further strengthening the notion of LPS-tolerance after repeated exposure.

TNF- $\alpha$ signaling can activate the transcription factor NF- $\kappa \mathrm{B}$, leading to the activation of mTOR, a prominent inhibitor of autophagy (Djavaheri-Mergny et al., 2006). We hypothesized that chronic LPS exposure would significantly upregulate TNF- $\alpha$ expression and potentially inhibit autophagy, leading to the promotion of apoptosis and necrosis, thus resulting in significantly larger infarct sizes. While this may occur in the general pathology of stroke, there do not appear to be significant differences in TNF- $\alpha$ expression between the saline and LPS exposed stroke animals.

Autophagy is generally regarded as a self-protective mechanism, which breaks down and recycles long-lived, damaged, or misfolded proteins and organelles (Wang et al., 2018b). Oxygen and nutrient deprivation can activate autophagy, and several studies have found that activation of autophagy during cerebral ischemia has neuroprotective benefits by degrading 
damaged structures and thereby limiting apoptosis (Wang et al., 2012; Papadakis et al., 2013). We assessed mRNA expression of several genes involved in the autophagy pathway; Beclin-1, ATG5, LC3a, and LC3b, which all have important functions in the formation and maturation of the autophagosome (Wang et al., 2018b). If post-stroke autophagy is being inhibited in the ipsilateral hemisphere of LPS exposed animals, damaged organelles and proteins are not being degraded. The accumulation of such damaged cellular contents can trigger apoptosis, or induce necrosis in these cells. This could contribute to the enlarged infarct we observed in the LPS exposed animals.

We also assessed levels of miRNAs previously implicated in stroke. miRNAs are small ( 22 nucleotides), non-coding RNAs that function as gene repressors by cleaving mRNAs, inhibiting mRNA translation, or targeting mRNAs for degradation (Ha and Kim, 2014). Up or downregulation of various miRNAs have been demonstrated to significantly impact stroke outcome. We chose to assess three miRNAs that have been previously implicated in stroke, including miR-155 (Caballero-Garrido et al., 2015; Pena-Philippides et al., 2016), miR-181a (Ouyang et al., 2012; Hough et al., 2018), and miR-181c (Ma et al., 2016). Contrary to our findings, several studies in the literature report observing increased post-stroke expression of miR-181a and miR-181c, and describe their detrimental role in stroke neuropathology (Ouyang et al., 2012; Ma et al., 2016; Hough et al., 2018). However, miR-181a (Zhu et al., 2017) and miR-181c (Zhang et al., 2012) negatively regulate TNF- $\alpha$ expression. Reduced expression of these miRNAs may allow for significant upregulation of TNF- $\alpha$ mRNA. TNF- $\alpha$ induces miR155 upregulation (Migita et al., 2017), and miR-155 targets several key players in autophagy (Wang et al., 2018a), including ATG5 (D’Adamo et al., 2016) and beclin-1. We observed significantly lower expression of miR-181a and -181c in the ipsilateral hemisphere of both the saline and LPS exposed stroke groups when compared to sham controls, and we also observed a striking increase in TNF- $\alpha$ mRNA in this region. Further, in the ipsilateral hemisphere, we observe significant elevation of miR-155, which can be upregulated by TNF- $\alpha$. As miR-155 targets several genes associated with autophagy, and we observe significant reductions in the ipsilateral hemisphere of all four genes, a link between these molecules is emerging, potentially leading to reduced autophagic functioning in the ipsilateral hemisphere of LPS exposed stroke 
animals. Utilizing miRNA overexpression or antagomir models would allow for more direct manipulation of these pathways to better aid in understanding their role in stroke outcomes. In addition, assessment of mRNA transcripts at the protein level would allow for direct understanding of miRNA-mediated gene repression.

DNA methylation is an epigenetic modification that can be inherited, as well as influenced by environmental factors. In recent years a growing interest in stroke-induced alterations of DNA methylation has surfaced (Qureshi and Mehler, 2010; Soriano-Tárraga et al., 2014, 2017). Segments ( $\geq 500$ base pairs) of DNA which contain $>55 \%$ cytosine and guanine nucleotides can be epigenetically modified via the methylation of cytosine residues. This mechanism is mediated by DNA methyl transferases (DNMTs), and inhibits gene transcription by preventing transcription factor binding (Krupinski et al., 2018). Aging, which is characterized by low-grade chronic inflammation (Franceschi et al., 2018), is the main risk factor for stroke, and is associated with global DNA hypomethylation (Johnson et al., 2012). Inflammation has also been directly associated with changes in DNA methylation (Abu-Remaileh et al., 2015; Maiuri et al., 2018). In animals chronically exposed to LPS, we observed significant downregulation of DNMT1 and DNMT3a in the ischemic hemisphere post-stroke. This downregulation should result in overexpression of numerous genes that have $\mathrm{CpG}$ islands. Future work should address the methylation status of the genome after intermittent exposures to LPS alone, as well as poststroke.

In conclusion, this study has shown that chronic systemic inflammation induced by intermittent injections of LPS can significantly increase cortical infarcts in an experimental stroke model. We show that in the brain, TNF- $\alpha$ mRNA expression is significantly elevated post-stroke, regardless of prior saline or LPS exposure. Further, we show that mRNA expression of autophagy genes is significantly downregulated in the ischemic hemisphere of LPS exposed mice. We propose that the mechanism by which significant increases in infarct volume occurs in the LPS exposed mice is by de-regulated autophagy, leading to significantly increased neuronal apoptosis.

Alternatively, in view of our observation of a downregulation of DNMT mRNA expression in 
the ipsilateral hemisphere of LPS exposed stroke animals, we also propose that changes in DNA methylation may also significantly contribute to increased infarct volume observed in this group. 


\section{Figure Legends}

Figure 4.1: Experimental timeline and study design. Prior to the start of the LPS (or saline) injection paradigm, baseline health scores were obtained. Four hours post-injection, health screen scores were measured to ensure animals exhibited sickness behaviors. To confirm recovery between LPS exposures, thirteen days post-injection (i.e. one day prior to the next injection) the health screen was again administered. LPS concentrations were doubled with each injection. Thirteen days after injection 5, the health screen was administered once more to verify that animals were no longer exhibiting sickness behavior. The following day, animals were subjected to a 70 minute tMCAO surgery, followed by a 24-hour reperfusion period, at the conclusion of which, animals were euthanized and tissues were collected.

Figure 4.2: LPS exposure induces an acute sickness response, from which animals recovered. Health screen scores for animals exposed to saline were grouped together as they did not significantly differ across time. There was a significant effect of LPS exposure on sickness behavior when compared to saline injected animals $(\mathrm{F} 10,157=12.69, \mathrm{p}<0.0001)$. Dunnett's post-hoc analysis indicated that when compared to saline-treated animals, animals exposed to LPS exhibited significant sickness behavior four hours post-injections 1, 2, and 4, while injection 5 trended towards significance $(\mathrm{p}=0.0667)$. Further, health sickness scores at the 13 day time point did not significantly differ from those in the saline-injected group, indicating that animals returned to normal health status. Data are expressed as mean \pm SEM; one-way ANOVA. **p < 0.01 and $* * * * \mathrm{p}<0.0001$.

Figure 4.3: Repeated LPS exposure significantly increases stroke infarct volume. A. Representative TTC stained coronal slices used to analyze infarct volume 24 hours post-stroke. B. Animals repeatedly exposed to LPS had significantly larger infarct volume than saline exposed mice in cortex and total hemisphere. Data are expressed as mean \pm SEM; brackets connect statistically significant groups; Student t test. $* \mathrm{p}<0.05$.

Figure 4.4: Stroke induces significant upregulation of TNF- $\alpha$ mRNA expression across the entire brain. A. Compared to sham animals, TNF- $\alpha$ mRNA was significantly upregulated in the 
contralateral hemisphere of animals subjected to a 70 minute tMCAO, regardless of prior saline or LPS exposure (F2, $25=28.33, \mathrm{p}<0.0001)$. B. Similarly, expression of TNF- $\alpha$ mRNA was significantly upregulated in the ipsilateral hemisphere of animals subjected to a 70 minute tMCAO when compared to sham $(\mathrm{F} 2,31=14.92, \mathrm{p}<0.0001)$. Data are expressed as mean \pm SEM; brackets connect statistically significant groups; $* * \mathrm{p}<0.01$ and $* * * * \mathrm{p}<0.0001$.

Figure 4.5: Autophagy-related genes are downregulated in the ipsilateral hemisphere of LPS exposed stroke animals. A. In the contralateral hemisphere, only saline injected animals had a significant reduction in Beclin-1 mRNA expression when compared to sham controls $(\mathrm{F} 2,26=$ 4.644, $\mathrm{p}=0.0189)$. B. Both saline and LPS injected animals expressed significantly lower levels of Beclin-1 in the ipsilateral hemisphere when compared to sham controls $(\mathrm{F} 2,31=5.457, \mathrm{p}<$ 0.0093). In the contralateral hemisphere, no significant differences in (C) ATG5, (E) LC3a, or (G) LC3b mRNA expression were observed. In the ipsilateral hemisphere, significant downregulation of (D) ATG5 (F2, $31=3.972, \mathrm{p}=0.0291)$, (F) LC3a (F2, $31=3.393, \mathrm{p}=$ 0.0465), and (H) LC3b mRNA expression was observed in the LPS exposed stroke animals when compared to sham controls. Data are expressed as mean \pm SEM; brackets connect statistically significant groups; $* \mathrm{p}<0.05$.

Figure 4.6: miRNA expression is altered 24-hours post-stroke. In the contralateral hemisphere, no significant changes in (A) miR-155, (C) miR-181a, or (E) miR-181c were observed. In the ipsilateral hemisphere, there was significant upregulation of $(\mathrm{B}) \operatorname{miR}-155(\mathrm{~F} 2,28=11.82, \mathrm{p}=$ 0.0002), and downregulation of (D) miR-181a (F2, $30=10.03, \mathrm{p}=0.0005)$ in both saline and LPS treated stroke animals, when compared to sham controls. Significant downregulation of (F) miR-181c $(\mathrm{F} 2,29=5.032, \mathrm{p}=0.0133)$ was observed only in the LPS treated animals when compared to sham controls. Data are expressed as mean \pm SEM; brackets connect statistically significant groups; $* \mathrm{p}<0.05, * * \mathrm{p}<0.01$ and $* * * \mathrm{p}<0.001$

Figure 4.7: DNMT mRNA expression is reduced post-stroke. DNMT1 mRNA expression was significantly reduced in both the $(\mathbf{A})$ contralateral $\left(\mathrm{F}_{2,26}=6.713, p=0.0045\right)$ and $(\mathbf{B})$ ipsilateral $\left(F_{2,30}=10.02, p=0.0005\right)$ hemispheres of the LPS exposed stroke animals, when compared to 
sham controls. Similarly, DNMT3a mRNA expression was significantly reduced in the (C) contralateral hemisphere of LPS stroke animals $\left(\mathrm{F}_{2,26}=4.388, p=0.0228\right)$, and in the $(\mathbf{D})$ ipsilateral hemisphere of both saline and LPS stroke animals $\left(F_{2,30}=9.661, p=0.0006\right)$. No significant changes in DNMT3b mRNA expression were observed $(\mathbf{E}, \mathbf{F})$. Data are expressed as mean \pm SEM; brackets connect statistically significant groups; $* * p<0.01$, ***p $<0.001$, and $* * * * p<0.0001$. 


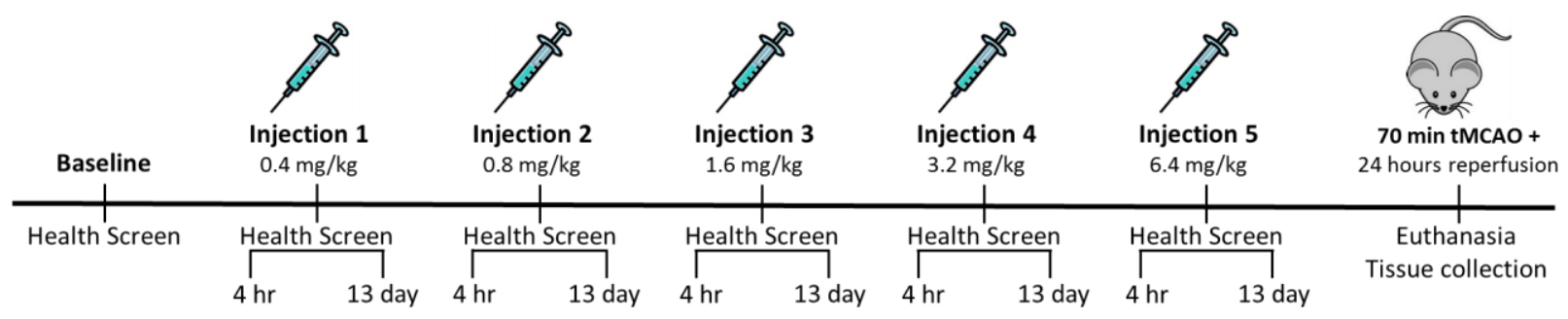

Figure 4.1 


\section{Post-Injection Sickness Behavior}

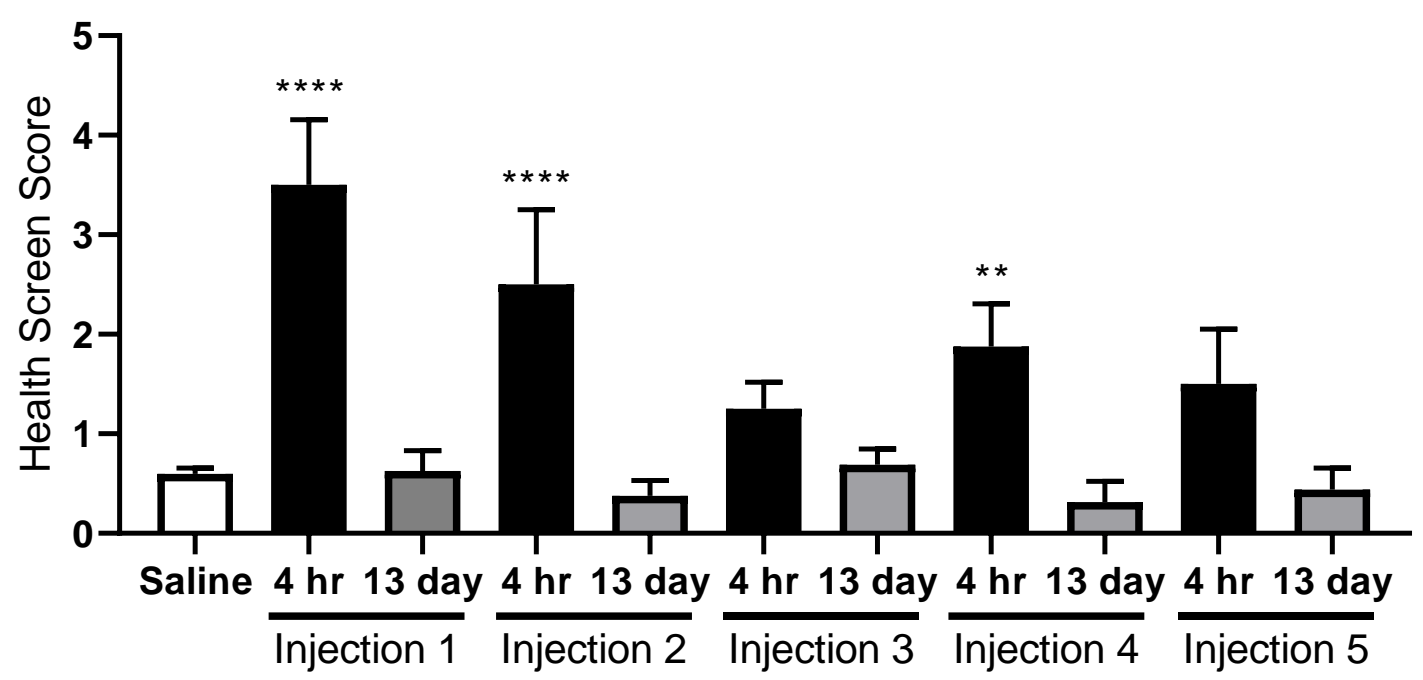

Figure 4.2 


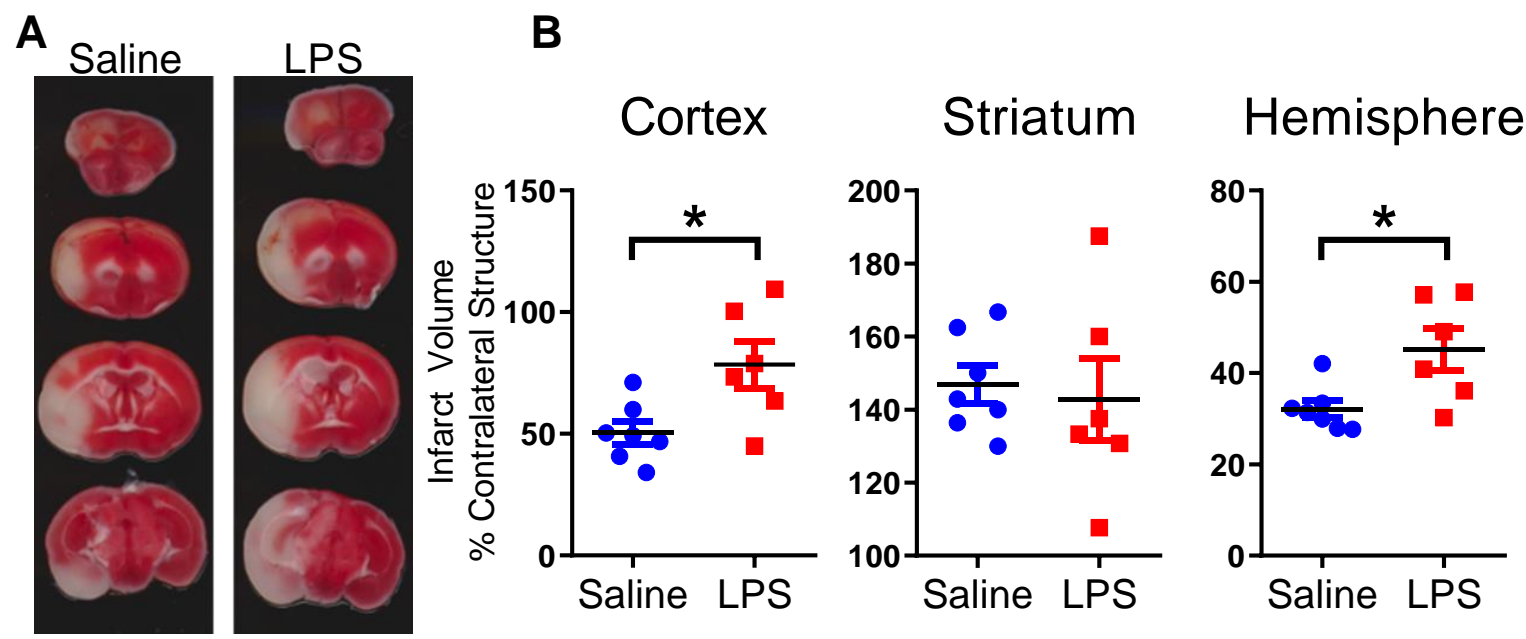

Figure 4.3 

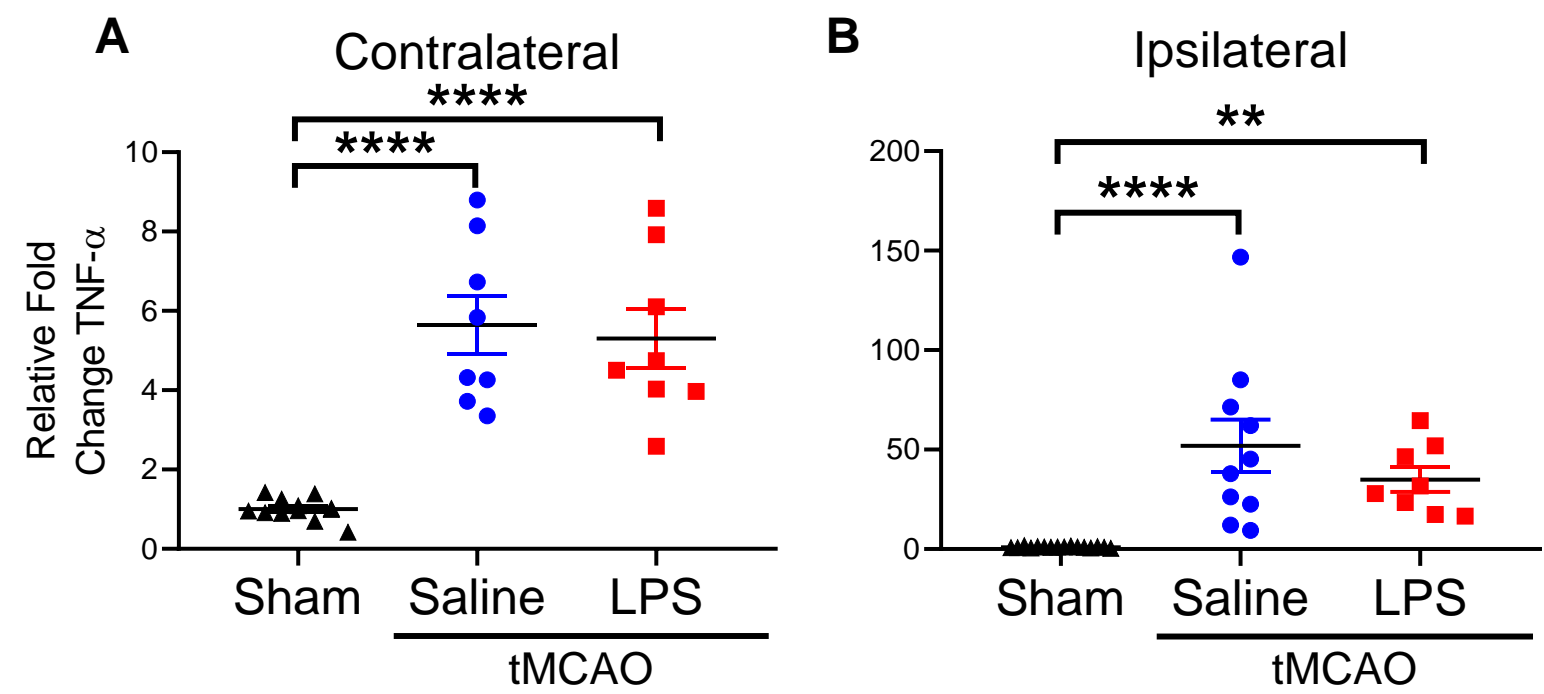

Figure 4.4 

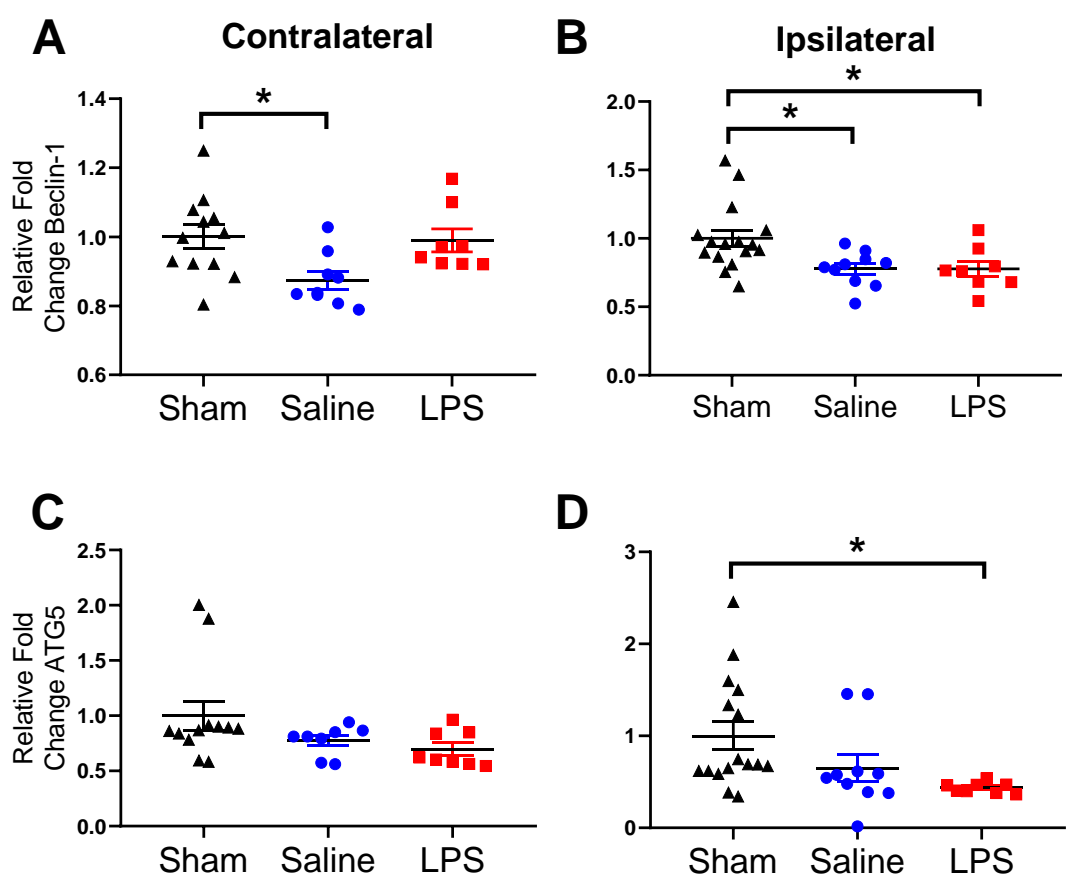

D

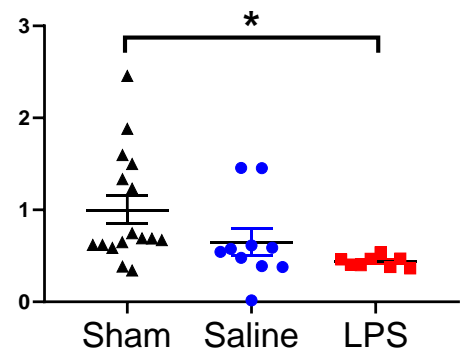

E

F
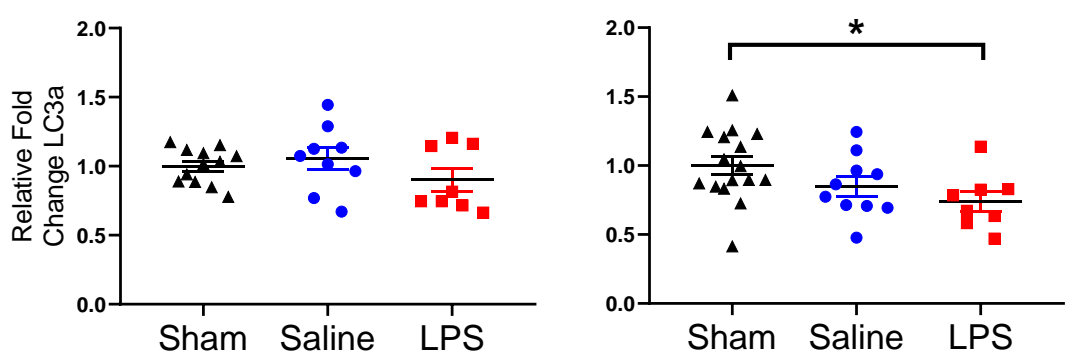

G

H
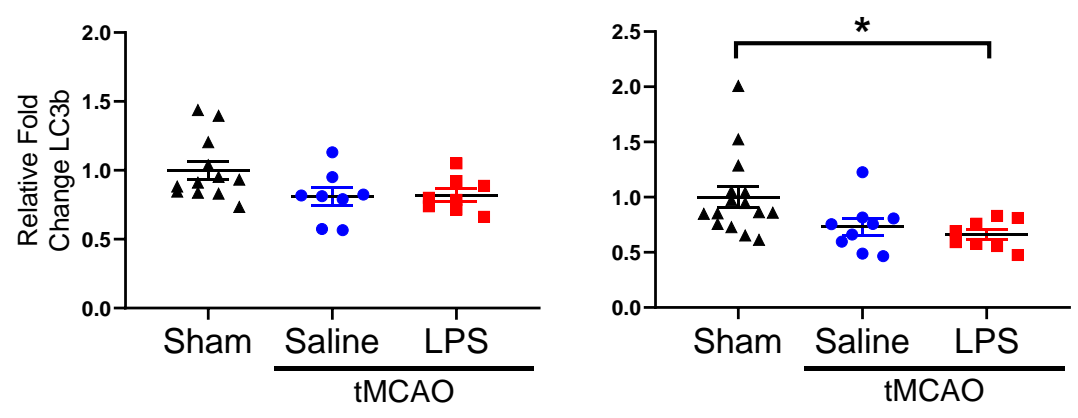

Figure 4.5 

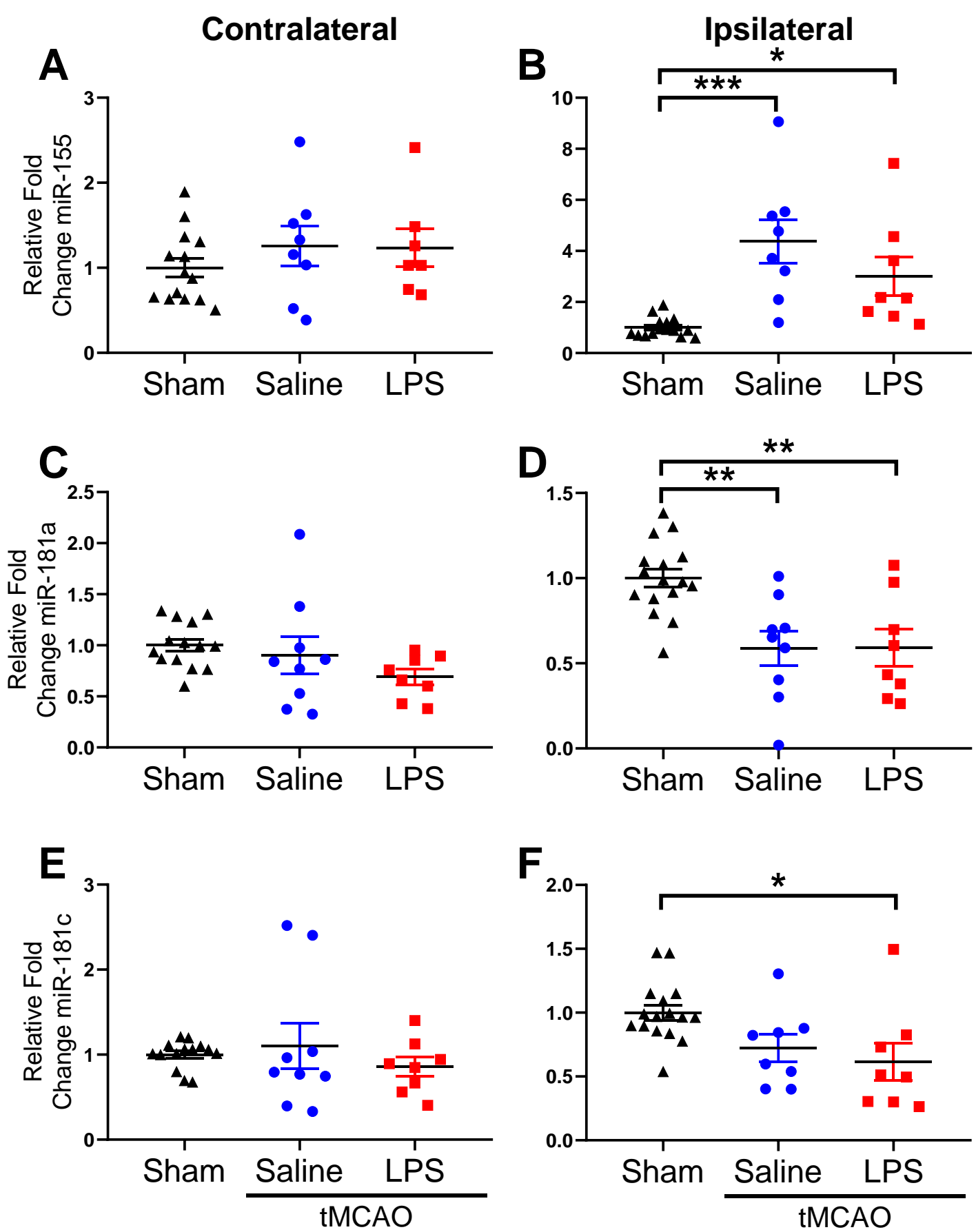

Figure 4.6 

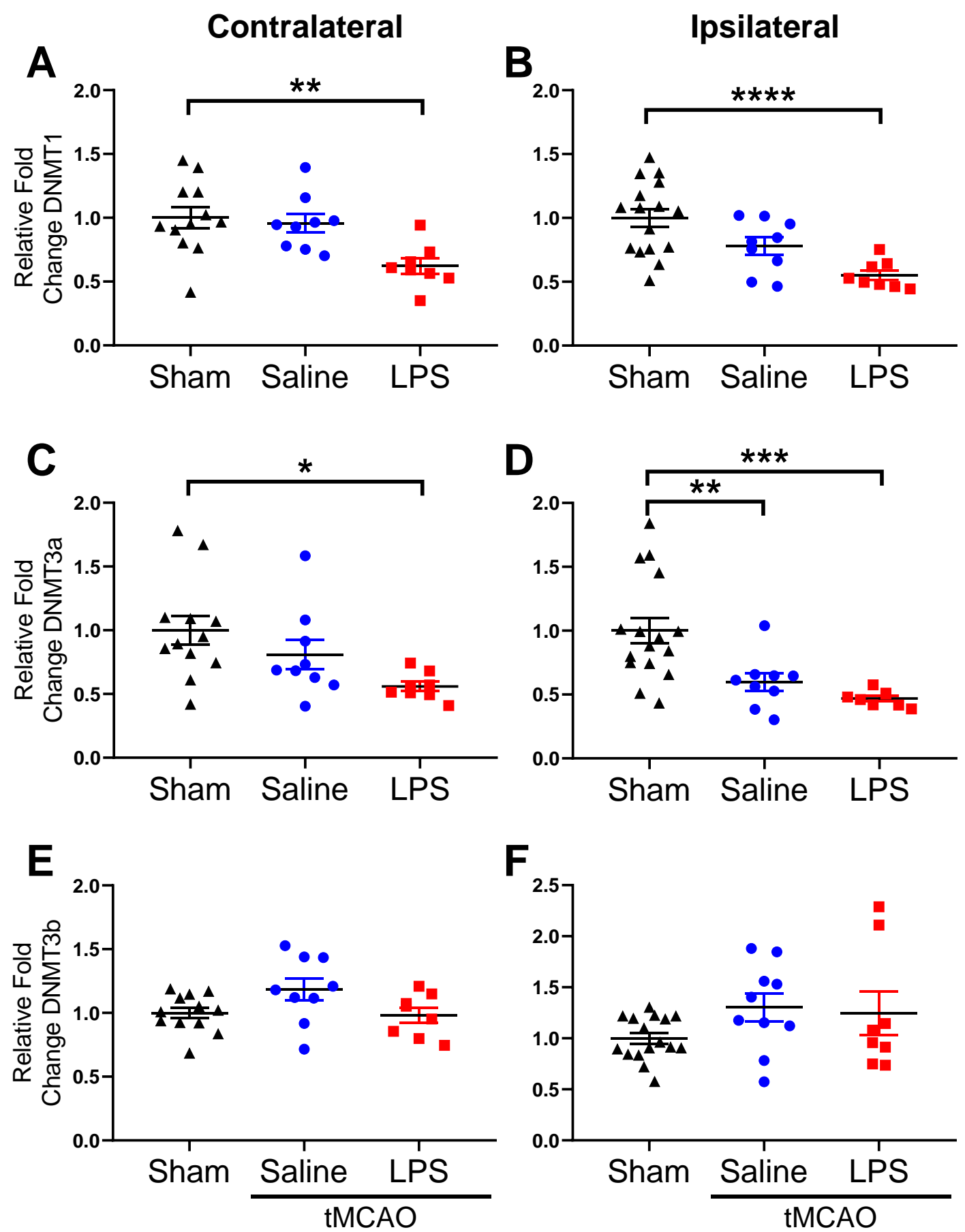

Figure 4.7 
Chapter 5

\section{Discussion}




\section{Discussion}

The immune and nervous systems are both very complex networks. Elucidating the numerous mechanisms that influence their crosstalk will undoubtedly assist in unveiling the etiology and pathophysiology of various diseases, including that of AD and stroke. Discovery of viable treatments and cures for such ailments is indeed on the horizon, as science and technology converge and provide researchers with more information and experimental techniques than ever before. In this dissertation, I have focused on the inflammatory mediators, TNF- $\alpha$ and LPS, and their role in inflammatory processes in the brain, with a focus on EV production, miRNA expression, mitochondrial dysfunction, and autophagy.

Through this body of work, I have found that direct exposure of TNF- $\alpha$ and LPS significantly alters the expression of miRNAs associated with mitochondrial dysfunction (miR-146a and miR34a) in a variety of CNS-related cell types. Indeed, overexpression of miR-146a significantly reduces cellular bioenergetic functioning, as does overexpression of miR-34a (Sarkar et al., 2016). Further, stimulation of cells with TNF- $\alpha$ or LPS significantly alters EV secretion and cargo, indicating that the communicative signaling packages (i.e. EVs) released by these stimulated cells are modified as a direct result of their environment. Additionally, EV uptake significantly alters recipient cell bioenergetics. Lastly, intermittent systemic exposure to LPS significantly increases stroke cortical infarct volume at 13 day post-stroke. After assessing expression of several mRNAs and miRNAs, we believe this effect is mediated through downregulation of autophagy and changes in DNA methylation.

\section{Inflammatory stimuli, EVs, and miRNAs}

Inflammation significantly alters EV secretion and miRNA expression profiles in various CNS cell types. Our studies indicate that 24-hours of TNF- $\alpha$ exposure significantly increases EV secretion from neurons. Several recent papers report similar findings after TNF- $\alpha$ exposure in astrocytes (Wang et al., 2017a; Chaudhuri et al., 2018) and brain endothelial cells (Dozio and Sanchez, 2017). Others have reported significant changes to EV profiles post-inflammatory insult (Yang et al., 2018), and it has been shown that EVs can mediate an immune response 
(Buzas et al., 2014). It is very likely that this secretory event occurring after exposure to an inflammatory stimulus is the first step in a mechanism by which cells signal to their neighbors that something is amiss, with EV-cell interaction being the next step in the process.

Previous work has shown that the EV-mediated transfer of miRNAs can alter protein expression in recipient cells. As such, we also assessed changes in miRNA expression post-insult, both within cells and their secreted EVs, and found that inflammatory stimuli significantly increase expression of miR-34a and miR-146a.

Some intriguing observations have been made regarding intracellular vs EV RNA profiles which warrant further introspection. It has been shown that larger EVs appear to have RNA profiles which more closely mirror the parent cells from which they are derived, while smaller EVs differ significantly from their cognate cells (Wei et al., 2017). As large EVs inherently have more luminal space, they may be predisposed to contain more molecules representative of the parent cell. On the other hand, small EVs have limited space, and may not be able to house many of the molecules found in their parent cells. Conversely, several groups have posited that differences in $\mathrm{EV}$ and cell RNA profiles represent differential sorting and secretion mechanisms, based on RNA sequence (Abels and Breakefield, 2016; Wei et al., 2017). Data from our studies provide evidence to support the notion of specific sorting mechanisms, however our data also suggest that this may rely on the cell and stimulus type.

Neuronal exposure to TNF- $\alpha$ for 24 hours does not alter intracellular expression of miR-34a, however there is a dose-dependent, significant increase in miR-34a expression in secreted EVs. Conversely, the same treatment conditions induced significant intracellular upregulation of miR146a, which appeared to reach a ceiling threshold, after which it was secreted in EVs in a dosedependent manner. We also present similar data in primary neurons exposed to increasing concentrations of A $\beta$; again, intracellular levels of miR-146a appear to reach a ceiling threshold, at which point it is then secreted in EVs. Initial interpretation of these data are in support of the concept that one miRNA, miR-146a, may rely on stochastic sorting mechanisms based on concentration and distribution throughout the cell, while another, miR-34a, may be preferentially 
secreted from cells upon its synthesis, perhaps to act as an intercellular signaling molecule. To complicate this idea however, we also have data from TNF- $\alpha$ exposed brain endothelial cells indicating that intracellular miR-146a levels are significantly reduced post-exposure, yet significantly increased in secreted EVs. However, when these cells are exposed to LPS, miR146a expression is significantly increased, both intracellularly and in secreted EVs. It is plausible that miRNA expression and sorting mechanisms may be activated in both cell and stimulus-type specific fashions. Determining whether or not these sorting mechanisms exist, if they are differentially affected by varying stimuli, and the cell specificity of this effect are areas of ongoing research in the EV field (Cha et al., 2015; McKenzie et al., 2016; Hinger et al., 2018).

Our studies indicate that both LPS and TNF- $\alpha$ exposure significantly increase the expression of miR-34a and miR-146a in vitro in various cell types of the CNS. Because of the proinflammatory cascade initiated by oxygen-glucose deprivation, we also assessed expression of these miRNAs in the brains of our sham and stroke animals. Interestingly, we found no significant changes in expression of either miRNA post-stroke (Figure A1). These data further indicate that miRNA expression may be heavily stimulus and context specific.

\section{The importance of cellular-specific RNA expression}

Knowing the cellular source of miRNA expression in tissue is also an important consideration for discussion. For example, miR-155 is significantly upregulated in the ischemic hemisphere of post-stroke LPS exposed animals. In neurons and their secreted EVs, TNF- $\alpha$ exposure significantly increases miR-155 expression in a pattern similar to that of miR-146a expression (Figure A2). Because expression of miRNAs in our animal studies was performed with whole brain tissue, we cannot accurately determine the source of miR-155. Other cell types, including microglia (Cardoso et al., 2012; Yin et al., 2017), have been shown to release miR-155 in response to inflammatory stimuli (Alexander et al., 2015).

A viable approach for determining cell-specific miRNA expression from tissue is to utilize fluorescence-activated cell sorting (FACS). By labeling neuron, microglial, astrocytic, and 
endothelial cell specific markers, we could sort tissue homogenates into cell-specific populations for downstream miRNA assessment (Guez-Barber et al., 2012). From this, we could determine the specific cell types implicated in disease pathogenesis.

The importance of cell-type determination of RNA expression can also be discussed in the context of mRNAs. We assessed expression of the autophagy genes Beclin-1, ATG5, LC3a, and LC3b, at the tissue level in sham and stroke animals. Significant downregulation of these genes was observed in the ischemic hemisphere of the LPS exposed animals, however we do not know which cells are being affected by the potential downregulation of autophagy. During ischemia, neurons are deprived of oxygen and glucose, and mitochondrial dysfunction occurs. Mitophagy is a quality control process by which damaged or stressed mitochondria are degraded via autophagy (Ding and Yin, 2012). This process has been implicated in the pathogenesis of stroke (Tang et al., 2016; Guan et al., 2018). Administration of the autophagy activator rapamycin, significantly increased mitophagic activity after stroke, and significantly reduced infarct volume (Li et al., 2014). Due to our observation of downregulated autophagy genes in the LPS exposed stroke animals, we believe that the significantly larger infarct volumes exhibited in this group may be due to reductions in autophagic capacity, specifically mitophagy. Downregulation of this pathway will lead to accumulation of damaged and stressed mitochondria, with subsequent release of cyt $c$ into the cytosol, thus triggering apoptosis (Hara et al., 1997; Liang et al., 2013).

However, due to the utilization of whole-tissue samples, as opposed to cell-specific analysis, we cannot definitively ascertain which cell type autophagy is downregulated in. Is this a whole-brain issue, occurring within all CNS cell types, or does it only occur in specific cells? Most commonly studied in this context are neurons, however microglia may also be negatively impacted by reductions in autophagy. Although the role of microglial autophagy in ischemia is unknown (Plaza-Zabala et al., 2017), a recent study suggests that inhibition of this process may over-sensitize microglia and cause an over-activation of the proinflammatory response (Ye et al., 2017). If our observations of downregulated autophagy genes are predominately occurring in microglia, the subsequent increases in infarct size we may actually be a result of enhanced inflammation, not direct cell death incurred from inhibited autophagy in neurons. For 
understanding the pathophysiology of autophagy in stroke, it would be very informative to delineate exactly which cell types may be experiencing autophagic downregulation.

There is a large push in the EV field to utilize these small, lipid bound vesicles as therapeutic agents for drug delivery (Di Rocco et al., 2016; Vader et al., 2016; Lv et al., 2018). As EVs can cross the $\mathrm{BBB}$, there is great interest in utilizing designer EVs to deliver therapeutic molecules to the brain (Neubert and Glumm, 2016; Krämer-Albers, 2017; Rufino-Ramos et al., 2017). Additionally, some groups are working to create EVs that can target cells specifically, based on specific protein markers on the EV and cell surface (Antes et al., 2018). If these types of treatments become a reality, it is plausible that EVs could be engineered to specifically target subsets of cells for delivery of therapeutic agents. In light of the discussion above, knowledge of the cell-source from which altered RNA expression is stemming would greatly aid in designing safe and effective EV-delivery therapies. For example, if autophagy was downregulated specifically in microglia, and not neurons, adoptive transfer of microglia-targeted-EVs containing mRNAs of the endogenously downregulated proteins (or the proteins themselves) could rescue autophagy, and reduce the exaggerated pro-inflammatory phenotype. Incorrectly targeting these EVs to neurons instead of microglia could result in over activation of autophagy in these cells and exacerbate cell death even further (Liu and Levine, 2015). Conducting experiments assessing gene expression changes at the cell-level are significantly more informative than those at the tissue level, and will provide better insight for the future development of therapeutic agents.

\section{miR-146a and Mitochondrial Function}

In our studies, we found that neural exposure to the cytokine TNF- $\alpha$ induces significant reductions in oxidative phosphorylation and glycolysis, which may be due to significant cell death. However, downregulation of cellular bioenergetic function may be due, in part, to the increased intracellular expression of miR-146a. The potential mitochondrial electron transport chain (ETC) targets of miR-146a include: NADH-ubiquinone oxidoreductase chain 1, NADH dehydrogenase 2, NADH dehydrogenase subunit 4, NADH:Ubiquinone Oxidoreductase Core 
Subunit 5, NADH:Ubiquinone Oxidoreductase Core Subunit 6, ATP Synthase Membrane Subunit 8, as well as phosphoglycolate phosphatase (PGK1) which is involved in glycolysis. In these studies, we have also shown that overexpression miR-146a significantly reduces oxidative phosphorylation and glycolysis in primary microglia. The ETC mRNA targets described above are all encoded by mitochondrial DNA (as opposed to being nuclear encoded), therefore, these mRNAs should be constitutively expressed in neural cells as well. Although we did not directly examine the effects of miR-146a overexpression in neural cells, it is likely that we would observe similar significant reductions in oxidative phosphorylation, as shown in microglia.

We have also shown that miR-146a expression is positively correlated with AD pathology, specifically amyloid angiopathy. Mitochondrial dysfunction leads to increases in APP processing towards the amyloidogenic pathway (i.e. producing A 342 ) (Gabuzda et al., 1994; Atamna and Frey, 2007; Swerdlow et al., 2014). Upregulation of miR-146a in the AD brain leads has been mechanistically linked to hyperphosphorylation of tau (Wang et al., 2016), which is the basis of Braak \& Braak staging of AD (Braak and Braak, 1991b). Our data also show a positive correlation between miR-146a expression and Braak \& Braak staging. Together, these data suggest that inflammation-induced expression of miR-146a is highly pathological and in part, mediates mitochondrial dysfunction occurring within $\mathrm{AD}$ and as such, this miRNA may be a potential therapeutic target in AD.

EVs have been shown to contain functionally active proteins, RNA, and DNA, which can exert functions in recipient cells. As such, studying how their internal cargo affects recipient cells upon uptake is an exciting area of research (Baj-Krzyworzeka et al., 2002; Lotvall and Valadi, 2007; Raposo and Stoorvogel, 2013). As neural communication is one of the basic tenants of cognition, it is surprising how few groups focus on neuron-to-neuron communication via EVs. We were interested in determining the effects of cytokine-induced neuronal-derived EVs on naïve recipient neurons. Our observation of significantly elevated expression of miR-146a and 34a prompted the hypothesis that EV-uptake would induce significant mitochondrial and glycolytic dysfunction in recipient cells. 
Exposing cells to TNF- $\alpha$ induced EVs actually resulted in significant upregulation of oxidative phosphorylation with a concurrent increases in ROS. The $10 \mathrm{ng} / \mathrm{ml} \mathrm{TNF-} \alpha$ induced EV group displayed the highest amount of peroxide generation (and a non-significant increase in superoxide). Therefore, it is plausible that the significant amount of cell death observed in this group may be mediated by cytochrome $c$ (cyt $c$ ). miR-146a and miR-34a downregulates expression of B-cell lymphoma 2 (Bcl-2), which is an anti-apoptotic protein which resides in the mitochondrial outer membrane (Rippo et al., 2014). Downregulation of Bcl-2 augments ROS formation (Zimmermann et al., 2007; Uraoka et al., 2011), and can contribute to loss of the mitochondrial membrane potential (Wang and Welsh, 2014). Although we did not observe significant loss of membrane potential, measurements of this parameter were highly variable only in cells exposed to EVs (control group was less variable). Additionally, proton leak was significantly elevated, likely in an attempt to maintain membrane potential. It is possible that EV-mediated transfer of miR-146a and miR-34a was affecting these cells via the downregulation of Bcl-2, and subsequent alterations of mitochondrial membrane potential.

In these experiments, we induced the production of neuronal-derived EVs via acute exposure to an inflammatory stimulus. In turn, recipient cells were acutely exposed to these EVs, and although they produced significant amounts of ROS as a result, we did not observe significant downregulation of oxidative phosphorylation as we had anticipated. However, chronic inflammation is characterized by the persistent presence of inflammatory stimuli. Therefore, in states of chronic exposure, transcription of NFאB dependent miRNAs may occur more consistently. Further, chronic inflammation may also induce persistent EV secretion, and due to elevated miRNA transcription, resultant EVs may contain higher copy numbers of these miRNAs. As such, persistent exposure to EVs induced by persistent inflammatory stimuli more accurately recapitulate what is occurring in the inflamed brain. Time-course studies in which cells are repeatedly exposed to inflammatory stimuli, or inflammation-induced EVs would be interesting endeavors to pursue, as they would more accurately recapitulate what is occurring in vivo. 


\section{Systemic Inflammation and Stroke Outcome}

Our in vivo studies provide novel insights by which intermittent systemic inflammation can significantly alter gene expression within the brain, and subsequently, stroke outcome. At 13 days post-recovery, animals intermittently exposed to LPS had significantly larger infarcts than those exposed to saline. Further, these animals experienced significant reductions in mRNA expression of autophagy and DNMT genes. These data suggest that even during periods of perceived health, infection burden may be heavily weighing on the ability to respond to a negative immunological insult, such as a stroke, by changing the epigenetic landscape.

Although many stroke risk factors have been identified, with recent infection being one of them, the full contribution of each to onset of stroke remains unknown. Some evidence suggests that ischemic preconditioning prior to stroke can reduce negative stroke outcomes by inducing tolerance and augmentation of cellular survival mechanisms (Koch and Gonzalez, 2013). Additionally, others have shown that systemic preconditioning via immune activation with the TLR4 agonist, LPS, 48 hours prior to experimental stroke significantly reduces infarct volume and suppresses neutrophil infiltration to the brain (Rosenzweig et al., 2004). Similar results were obtained when low-doses of LPS $(0.05-0.2 \mathrm{mg} / \mathrm{kg})$ were chronically administered for three consecutive days prior to stroke (Rosenzweig et al., 2007). It appears that most LPS preconditioning studies utilize short-term paradigms. Indeed, the neuroprotective effects appear to only be relevant if LPS is administered within 1-7 days of experimental stroke (Stevens et al., 2008). In line with this, previous work from our laboratory demonstrated that administration of LPS 30 minutes prior to experimental stroke significantly exacerbated stroke infarct volume (Doll et al., 2015b). We present here our novel findings of intermittent LPS exposure on stroke infarct volume, which is more reflective of the infection burden experienced by a human; most humans do not only experience one illness during their lifetime, nor do they usually experience all of them over the course of several days. 
LPS tolerance has been studied for decades, however the mechanism by which it occurs has yet to be elucidated (López-Collazo and del Fresno, 2013). Data from our health screen assessment confer that systemic tolerance was achieved with repeated LPS exposure, as sickness behavior scores diminished with each injection. However, our infarct data are in strict contrast to much of the previously published LPS tolerance/preconditioning work.

Interestingly, TNF- $\alpha$ has been implicated in LPS preconditioning. After three days of low-dose systemic exposure to LPS, plasma TNF- $\alpha$ was significantly downregulated, as was TNFR1 in the cortex of the LPS exposed animals (Rosenzweig et al., 2007). Although we did not assess these parameters in our study, we did observe lower levels of TNF- $\alpha$ mRNA expression in the brains of LPS exposed stroke mice when compared to saline (although this was not statistically significant). If TNFR1 is indeed downregulated in the cortices of LPS exposed animals, this may explain why we observed lower expression levels of TNF- $\alpha$ mRNA. As TNFR1 signaling activates NF-kB, transcription of pro-inflammatory cytokines, including TNF- $\alpha$, is induced. This may create a positive feedback cycle; however if TNFR1 is downregulated, this cycling is blunted and less TNF- $\alpha$ expression is observed as a result.

Understanding how systemic immune activation can influence the pathophysiology of stroke is of great clinical relevance. In the current study, we utilized a bacterial mimetic, LPS to induce an immune response. The majority of stroke patients are elderly, and people over the age of 65 tend to experience more viral than bacterial infections (Leng and Goldstein, 2010). This may be due, in part, to the slow rate of bacterial mutation, which allows individuals to build immunity towards a greater number of bacterial pathogens throughout their lifespan. Viruses on the other hand, mutate rapidly, and the elderly typically have less-effective immune responses, making them more susceptible to viral infections (Leng and Goldstein, 2010). To be more clinically relevant, future studies could induce an immune response with polyinosinic:polycytidylic acid (poly I:C) to mimic a viral infection. In our immune activation paradigm, we induced a very mild sickness, from which animals were able to recover. This was akin to a human experiencing a mild cold about once every two years. If the number of times a person catches a cold throughout 
their lifetime significantly impacts the pathophysiology of stroke, we as a society need to rethink the way we view illnesses.

As we observed significant changes in gene expression, it is highly likely that other genes not measured in this experiment were also altered in the LPS exposed stroke animals. Further investigation is warranted here, as we did not assess changes in gene expression in a LPS-sham group of animals. Additionally, as we observed downregulation of DNMTs, it is also possible that changes in DNA methylation have occurred in the LPS exposed stroke animals. Acetylation of histones prevents DNA transcription; inflammation has been shown to reduce acetylation and allow for aberrant expression of normally repressed genes (Khangura et al., 2017). Although not assessed in the current study, this type of epigenetic modification may also have played a role in exacerbating infarct volumes in the LPS exposed stroke animals. Again, assessment of these parameters in LPS-sham animals is necessary to obtain a better understanding of the mechanisms at play here. We cannot say for certain that LPS alone has triggered these changes in gene expression, as these alterations may be a combinatorial result of intermittent LPS exposure and stroke.

\section{Inflammation and Systemically Circulating EVs}

Recent evidence suggests that both systemic and nervous system inflammation alter circulating EVs, and these EVs can exert immunologic effects on recipient cells/organisms. Monocytedifferentiated macrophages were exposed to EVs isolated from the serum of acute stroke patients; after a four-hour exposure period, macrophages incubated with stroke patient EVs were activated, and TNF- $\alpha$ mRNA was significantly upregulated (Couch et al., 2017a).

Experimentally induced inflammation via peripheral LPS challenge significantly increased circulating EVs (Li et al., 2018). These vesicles did not express endotoxin, but when injected into naïve animals, were able to induce neuroinflammation as evidenced by elevated microglial activation, astrogliosis, increased pro-inflammatory cytokine expression, and significant upregulation of miR-155 (Li et al., 2018). In a similar experiment, CNS injury was induced by 
striatal injection of IL-1B, followed by isolation of circulating EVs (Couch et al., 2017b). These EVs were then injected into naïve animals, which subsequently expressed sickness behavior and upregulated expression of TNF- $\alpha$ mRNA in the periphery (Couch et al., 2017b). Together, these data suggest that inflammation significantly alters EV concentrations, and these EVs have the capacity to significantly alter health status, and likely contribute to the immune response.

We evaluated the circulating plasma EV profile of animals intermittently injected with LPS or saline 24 hours post-stroke, as well as profiles from sham surgery animals (Figure A3). Surprisingly, we found that stroke significantly reduced EV concentrations when compared to sham controls (Figure A3A). We also made an interesting observation, noting that there were significant differences in EV concentrations between the saline and LPS injected stroked animals, with LPS injected animals having significantly higher concentrations of EVs than the saline animals. EV size distributions were also significantly affected by stroke (Figure A3B); sham animal EVs were mostly concentrated at $150 \mathrm{~nm}$, while stroke EVs were more concentrated between 200-350 nm. Differences in EV diameter may represent varying EV populations, as exosomes are believed to be $\leq 150 \mathrm{~nm}$, and MVs can be up to $1,000 \mathrm{~nm}$. It is possible that stroke reduces the amount of bona fide exosomes released into circulation, and enhances membrane shedding of MVs.

Our data are in direct opposition of the previous studies detailed above in which LPS and stroke increase circulating EV concentrations. An important caveat to point out is that the above mentioned studies isolated EVs from serum, while we utilized plasma. To isolate serum, blood must first be allowed to clot. During this process, platelets secrete substantial amounts of EVs (Gemmell et al., 1993) which may constitute over half of all EVs detected in a serum preparation (Witwer et al., 2013). Indeed, thrombogenic activators have been shown to be extremely potent inducers of platelet-EV secretion (Aatonen et al., 2014, 2017). Additionally, platelets are activated post-stroke (Marquardt et al., 2002; Cevik et al., 2016). The combination of these two factors (thrombogenic activation and ischemic stroke) may significantly increase EV 
concentrations, post-blood collection. As such, plasma is the preferred fluid for EV isolation from blood (Witwer et al., 2013), and may serve as a more accurate representation of circulating blood EV profiles.

An additional confounding factor that differentiates our study from the two stroke studies described above are that mice do not spontaneously experience strokes, they must be experimentally induced. Stroke risk factors like hypertension (Erne et al., 1985), smoking (Erikssen et al., 1977; Ghahremanfard et al., 2015), diet (McEwen, 2014), and weight (Santilli et al., 2012) have been shown to influence platelet number, adhesiveness, and microparticle (i.e. $\mathrm{EV})$ secretion. If all of these variables increase, and activate platelets, these patients may have significantly elevated levels of platelet-derived circulating EVs to begin with. After the ischemic event, and after coagulation occurs for serum isolation, these concentrations may rise substantially.

Regardless of the discrepancies in EV concentrations, an intriguing follow-up experiment would be to isolate EVs from four groups of animals (intermittent LPS, intermittent saline, intermittent LPS + stroke, intermittent saline + stroke) and tease out the effects of chronic immune activation vs. stroke on circulating EV concentrations. Further, we could then inject naïve animals with these EVs to determine how these EVs affect sickness behavior, systemic inflammation, and neuroinflammation in the recipient animals. As EVs are postulated to cross the BBB (Matsumoto et al., 2018), we could also assay the effects of these isolated EVs on microglia and neurons in vitro, and examine their potential inflammatory effects.

\section{Summary}

My dissertation provides novel data demonstrating that various inflammatory stimuli (LPS, TNF$\alpha$, and $A \beta$ ) significantly alter miR-146a expression in the secreted EVs of neurons, microglia, and brain endothelial cells. We also show that overexpression of this miRNA significantly 
downregulates mitochondrial function, which may underlie mitochondrial dysfunction observed in neuroinflammatory diseases. Further, we describe an understudied mechanism by which neuron-to-neuron communication occurs during periods of inflammation, and for the first time, provide data on the functional outcomes of neural uptake of inflammation-induced EVs on cellular bioenergetics. In vivo evidence from our animal studies suggest that intermittent systemic LPS exposure significantly increases stroke infarct size. The underlying mechanisms by which this phenomenon occurs are likely related to downregulation of autophagy or changes in DNA methylation. Together, these data suggest that inflammation is a powerful modulator of genetic alterations within the brain. 


\section{Appendix}
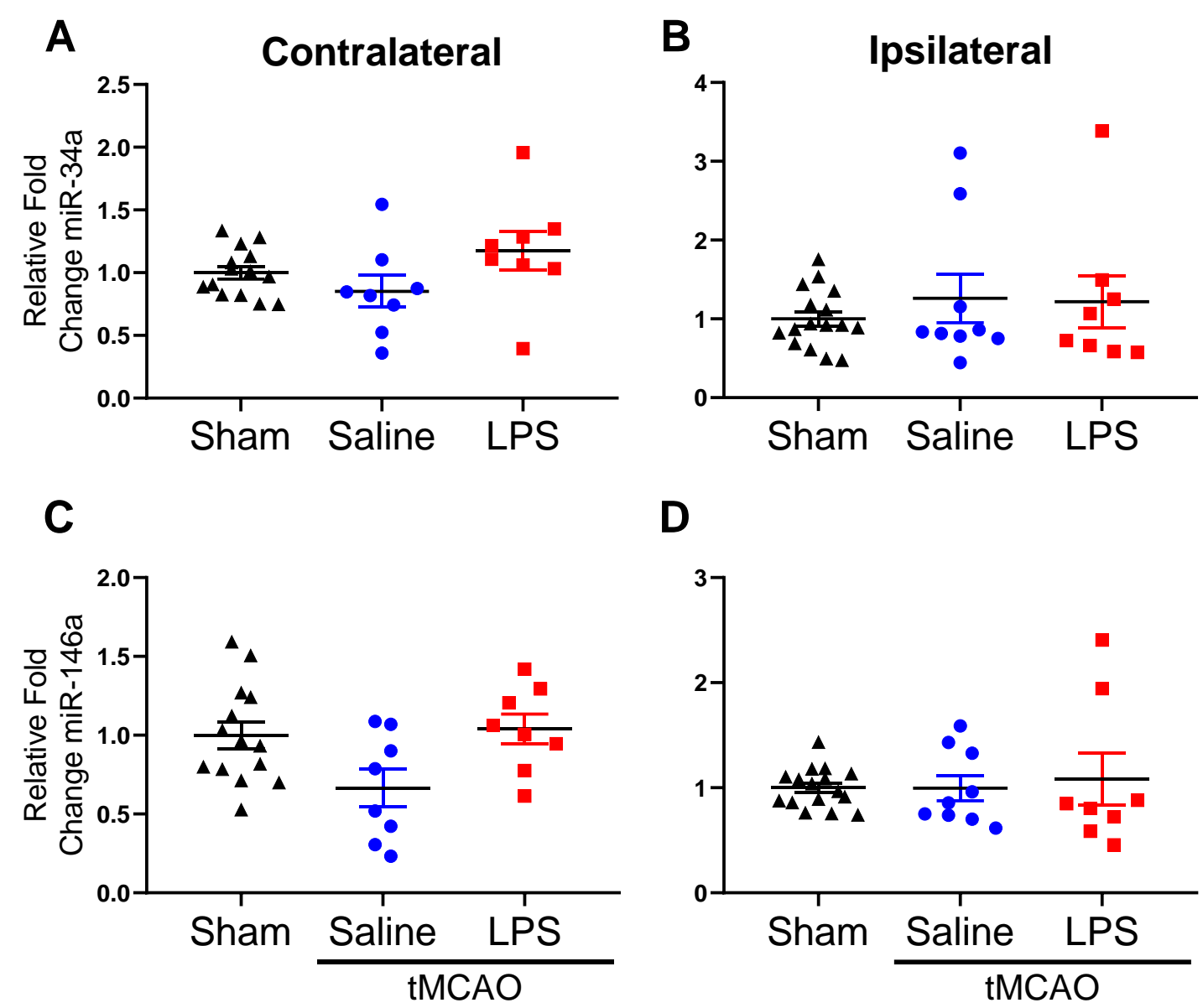

D

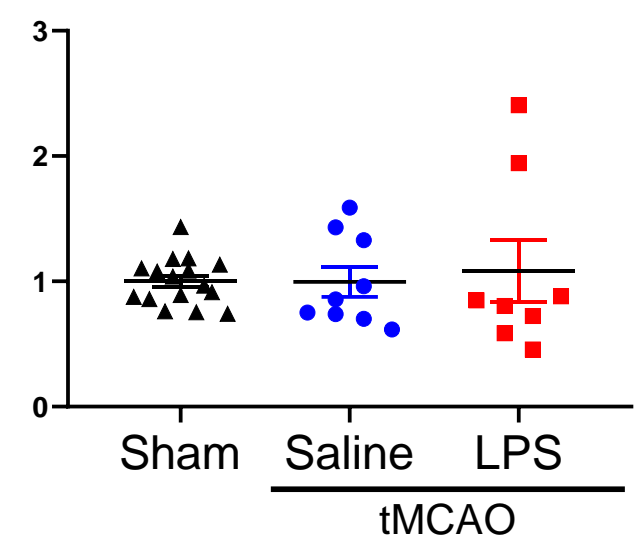

Figure A1. Post-stroke expression of miR-34a and miR-146a. There are no significant changes in miR-34a expression in the (A) contralateral or (B) ipsilateral hemisphere. Similarly, there are no significant changes in miR-146a expression in the (A) contralateral or (B) ipsilateral hemisphere. Data are expressed as mean \pm SEM. 

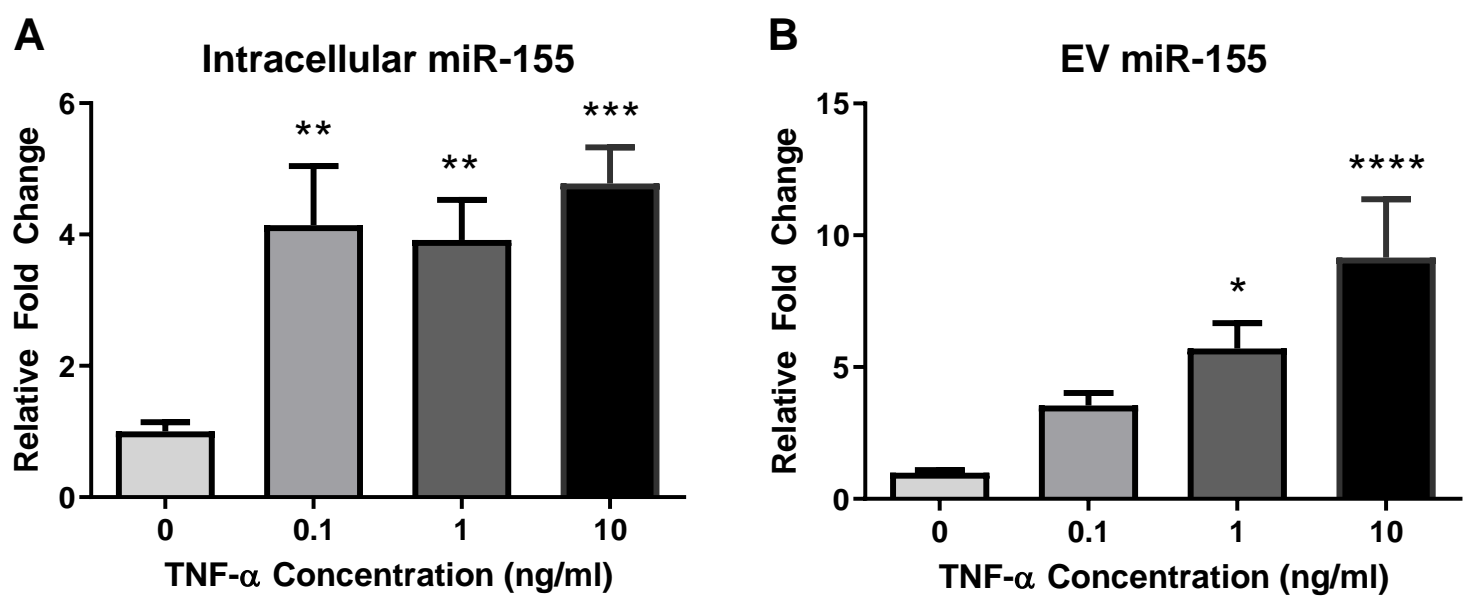

Figure A2. Intracellular and EV miR-155 expression. HT-22 cells were exposed to TNF- $\alpha$ for 24 hours and expression of miR-155 was profiled both intracellularly and within the secreted EVs. Intracellular expression was significantly elevated in all TNF- $\alpha$ exposure groups $\left(\mathrm{A}_{3} ; \mathrm{F}_{3,31}=\right.$ 7.504, $p=0.0006)$. EV miR-155 expression was significantly elevated at the two highest TNF- $\alpha$ exposures $\left(\mathrm{B} ; \mathrm{F}_{3,31}=8.762, p=0.0002\right)$. Data are expressed as mean $\pm \mathrm{SEM}$; brackets connect statistically significant groups; $* \mathrm{p}<0.05,{ }^{*} \mathrm{p} p<0.01, * * * \mathrm{p}<0.001, * * * * \mathrm{p}<0.0001$. 

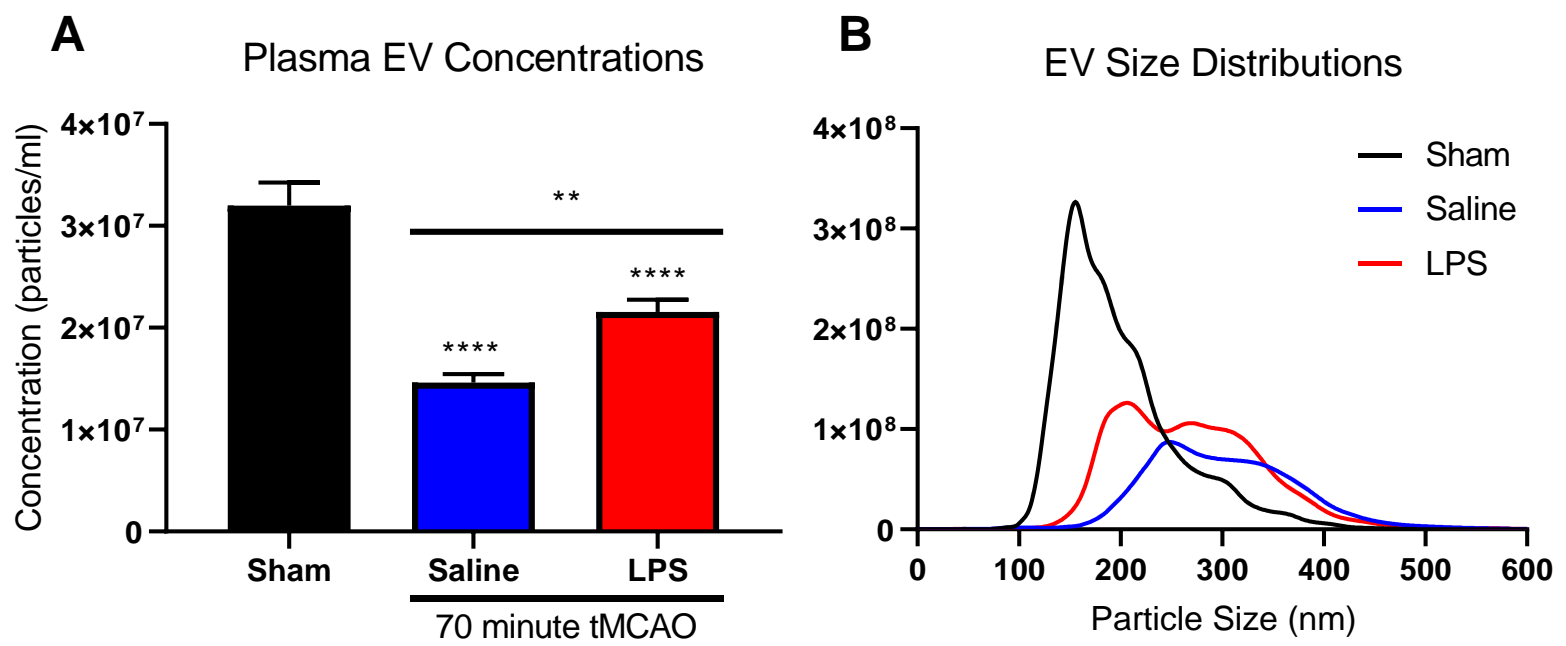

Figure A3. Post-stroke plasma EV concentrations. EVs were isolated from the plasma of stroke animals at 24 hours reperfusion. Stroke surgery significantly reduced circulating plasma EV concentrations $\left(\mathbf{A} ; \mathrm{F}_{2,2997}=31.34, p<0.0001\right)$. Dunnett's post-hoc analysis significantly indicated that there was a significant difference in EV concentration between saline and LPS treated animals, with LPS animals having significantly higher circulating levels. Stroke also substantially altered the size distribution profiles of EVs (B). Sham animal EVs were mostly concentrated at $150 \mathrm{~nm}$, while stroke EVs were more concentrated between 200-350 nm. Data are expressed as mean \pm SEM; brackets connect statistically significant groups; $* * p<0.01$, $* * * * \mathrm{p}<0.0001$. 


\section{References}

Aatonen M, Valkonen S, Böing A, Yuana Y, Nieuwland R, Siljander P (2017) Isolation of Platelet-Derived Extracellular Vesicles. In: Methods in molecular biology (Clifton, N.J.), pp 177-188 Available at: http://www.ncbi.nlm.nih.gov/pubmed/27943214 [Accessed January $6,2019]$.

Aatonen MT, Ohman T, Nyman TA, Laitinen S, Grönholm M, Siljander PR-M (2014) Isolation and characterization of platelet-derived extracellular vesicles. J Extracell vesicles 3 Available at: http://www.ncbi.nlm.nih.gov/pubmed/25147646 [Accessed January 6, 2019]. Abels ER, Breakefield XO (2016) Introduction to Extracellular Vesicles: Biogenesis, RNA Cargo Selection, Content, Release, and Uptake. Cell Mol Neurobiol 36:301-312 Available at: http://www.ncbi.nlm.nih.gov/pubmed/27053351 [Accessed July 26, 2018].

Abu-Remaileh M, Bender S, Raddatz unter, Ansari I, Cohen D, Gutekunst J, Musch T, Linhart H, Breiling A, Pikarsky E, Bergman Y, Lyko F (2015) Tumor and Stem Cell Biology Chronic Inflammation Induces a Novel Epigenetic Program That Is Conserved in Intestinal Adenomas and in Colorectal Cancer. Available at: http://cancerres.aacrjournals.org/ [Accessed January 3, 2019].

Aggarwal BB (2003) Signalling pathways of the TNF superfamily: a double-edged sword. Nat Rev Immunol 3:745-756 Available at: http://www.ncbi.nlm.nih.gov/pubmed/12949498 [Accessed January 1, 2019].

Aguzzi A, Rajendran L (2009) The Transcellular Spread of Cytosolic Amyloids, Prions, and Prionoids. Neuron 64:783-790 Available at: http://www.ncbi.nlm.nih.gov/pubmed/20064386 [Accessed January 5, 2019].

Akiyama H et al. (2000a) Inflammation and Alzheimer's disease. Neurobiol Aging 21:383-421 Available at: http://www.ncbi.nlm.nih.gov/pubmed/10858586 [Accessed August 12, 2017].

Akiyama H, Arai T, Kondo H, Tanno E, Haga C, Ikeda K (2000b) Cell mediators of inflammation in the Alzheimer disease brain. Alzheimer Dis Assoc Disord 14 Suppl 1:S4753 Available at: http://www.ncbi.nlm.nih.gov/pubmed/10850730 [Accessed August 4, 2017].

Akiyama H, Schwab C, Kondo H, Mori H, Kametani F, Ikeda K, McGeer PL (1996) Granules in glial cells of patients with Alzheimer's disease are immunopositive for C-terminal sequences of beta-amyloid protein. Neurosci Lett 206:169-172 Available at: http://www.ncbi.nlm.nih.gov/pubmed/8710178 [Accessed August 4, 2017].

Alexander M, Hu R, Runtsch MC, Kagele DA, Mosbruger TL, Tolmachova T, Seabra MC, Round JL, Ward DM, O'Connell RM (2015) Exosome-delivered microRNAs modulate the inflammatory response to endotoxin. Nat Commun 6:7321 Available at: http://www.ncbi.nlm.nih.gov/pubmed/26084661 [Accessed August 23, 2018].

Alexander M, Ramstead AG, Bauer KM, Lee S-H, Runtsch MC, Wallace J, Huffaker TB, Larsen DK, Tolmachova T, Seabra MC, Round JL, Ward DM, O'Connell RM (2017) Rab27Dependent Exosome Production Inhibits Chronic Inflammation and Enables Acute Responses to Inflammatory Stimuli. J Immunol 199:3559-3570 Available at: http://www.ncbi.nlm.nih.gov/pubmed/28978688 [Accessed April 23, 2018].

Alexandrov PN, Dua P, Hill JM, Bhattacharjee S, Zhao Y, Lukiw WJ (2012) microRNA (miRNA) speciation in Alzheimer's disease (AD) cerebrospinal fluid (CSF) and 
extracellular fluid (ECF). Int J Biochem Mol Biol 3:365-373 Available at:

http://www.ncbi.nlm.nih.gov/pubmed/23301201 [Accessed August 23, 2018].

Alexandrov PN, Dua P, Lukiw WJ (2014) Up-Regulation of miRNA-146a in Progressive, Age-

Related Inflammatory Neurodegenerative Disorders of the Human CNS. Front Neurol 5:181

Available at: http://www.ncbi.nlm.nih.gov/pubmed/25324823 [Accessed August 25, 2018].

Alvarez JI, Katayama T, Prat A (2013) Glial influence on the blood brain barrier. Glia 61:19391958.

Alzheimer's Association (2016) 2016 Alzheimer's disease facts and figures. Alzheimers Dement 12:459-509 Available at: http://www.ncbi.nlm.nih.gov/pubmed/27570871 [Accessed January 1, 2019].

Anand S, Foot N, Ang C-S, Gembus KM, Keerthikumar S, Adda CG, Mathivanan S, Kumar S (2018) Arrestin-Domain Containing Protein 1 (Arrdc1) Regulates the Protein Cargo and Release of Extracellular Vesicles. Proteomics 18:1800266 Available at: http://www.ncbi.nlm.nih.gov/pubmed/30035390 [Accessed October 29, 2018].

Antes TJ, Middleton RC, Luther KM, Ijichi T, Peck KA, Liu WJ, Valle J, Echavez AK, Marbán E (2018) Targeting extracellular vesicles to injured tissue using membrane cloaking and surface display. J Nanobiotechnology 16:61 Available at: https://jnanobiotechnology.biomedcentral.com/articles/10.1186/s12951-018-0388-4 [Accessed January 6, 2019].

Antonucci F, Turola E, Riganti L, Caleo M, Gabrielli M, Perrotta C, Novellino L, Clementi E, Giussani P, Viani P, Matteoli M, Verderio C (2012) Microvesicles released from microglia stimulate synaptic activity via enhanced sphingolipid metabolism. EMBO J 31:1231-1240 Available at: http://www.ncbi.nlm.nih.gov/pubmed/22246184 [Accessed January 5, 2019].

Ard MD, Cole GM, Wei J, Mehrle AP, Fratkin JD (1996) Scavenging of Alzheimer's amyloid ?protein by microglia in culture. J Neurosci Res 43:190-202 Available at:

http://doi.wiley.com/10.1002/\%28SICI\%2910974547\%2819960115\%2943\%3A2\%3C190\%3A\%3AAID-JNR7\%3E3.0.CO\%3B2-B

[Accessed August 12, 2017].

Atamna H, Frey WH (2007) Mechanisms of mitochondrial dysfunction and energy deficiency in Alzheimer's disease. Mitochondrion 7:297-310 Available at: http://www.ncbi.nlm.nih.gov/pubmed/17625988 [Accessed January 7, 2019].

Baik SH, Kang S, Son SM, Mook-Jung I (2016) Microglia contributes to plaque growth by cell death due to uptake of amyloid $\beta$ in the brain of Alzheimer's disease mouse model. Glia 64:2274-2290 Available at: http://doi.wiley.com/10.1002/glia.23074 [Accessed August 9, 2018].

Baj-Krzyworzeka M, Majka M, Pratico D, Ratajczak J, Vilaire G, Kijowski J, Reca R, Janowska-Wieczorek A, Ratajczak MZ (2002) Platelet-derived microparticles stimulate proliferation, survival, adhesion, and chemotaxis of hematopoietic cells. Exp Hematol 30:450-459 Available at: http://ac.els-cdn.com/S0301472X02007919/1-s2.0-

S0301472X02007919-main.pdf?_tid=35f45608-761d-11e7-acfa00000aab0f27\&acdnat=1501525530_f1d973d0680591a69d4cc386f6b56935 [Accessed July 31, 2017].

Balaban RS, Nemoto S, Finkel T (2005) Mitochondria, Oxidants, and Aging. Cell 120:483-495 Available at: https://www.sciencedirect.com/science/article/pii/S0092867405001091 
[Accessed January 2, 2019].

Baltan S (2015) Can lactate serve as an energy substrate for axons in good times and in bad, in sickness and in health? Metab Brain Dis 30:25-30 Available at: http://www.ncbi.nlm.nih.gov/pubmed/25034458 [Accessed January 5, 2019].

Balusu S, Van Wonterghem E, De Rycke R, Raemdonck K, Stremersch S, Gevaert K, Brkic M, Demeestere D, Vanhooren V, Hendrix A, Libert C, Vandenbroucke RE (2016) Identification of a novel mechanism of blood-brain communication during peripheral inflammation via choroid plexus-derived extracellular vesicles. EMBO Mol Med 8:11621183 Available at: http://www.ncbi.nlm.nih.gov/pubmed/27596437 [Accessed August 10, 2018].

Barghorn S, Nimmrich V, Striebinger A, Krantz C, Keller P, Janson B, Bahr M, Schmidt M, Bitner RS, Harlan J, Barlow E, Ebert U, Hillen H (2005) Globular amyloid beta-peptide142 oligomer - a homogenous and stable neuropathological protein in Alzheimer's disease. J Neurochem 95:834-847 Available at: http://www.ncbi.nlm.nih.gov/pubmed/16135089 [Accessed August 9, 2018].

Barone FC, Arvin B, White RF, Miller A, Webb CL, Willette RN, Lysko PG, Feuerstein GZ (1997) Tumor necrosis factor-alpha. A mediator of focal ischemic brain injury. Stroke 28:1233-1244 Available at: http://www.ncbi.nlm.nih.gov/pubmed/9183357 [Accessed January 3,2019$]$.

Bas-Orth C, Tan Y-W, Lau D, Bading H (2017) Synaptic Activity Drives a Genomic Program That Promotes a Neuronal Warburg Effect. J Biol Chem 292:5183-5194 Available at: http://www.ncbi.nlm.nih.gov/pubmed/28196867 [Accessed January 4, 2019].

Batagov AO, Kuznetsov VA, Kurochkin I V (2011) Identification of nucleotide patterns enriched in secreted RNAs as putative cis-acting elements targeting them to exosome nano-vesicles. BMC Genomics 12:S18 Available at: http://www.ncbi.nlm.nih.gov/pubmed/22369587 [Accessed August 2, 2017].

Behm-Ansmant I, Rehwinkel J, Doerks T, Stark A, Bork P, Izaurralde E (2006) mRNA degradation by miRNAs and GW182 requires both CCR4:NOT deadenylase and DCP1:DCP2 decapping complexes. Genes Dev 20:1885-1898 Available at: http://www.ncbi.nlm.nih.gov/pubmed/16815998 [Accessed January 2, 2019].

Bell MD, Taub DD, Perry VH (1996) Overriding the brain's intrinsic resistance to leukocyte recruitment with intraparenchymal injections of recombinant chemokines. Neuroscience 74:283-292 Available at: http://www.ncbi.nlm.nih.gov/pubmed/8843093 [Accessed August $3,2017]$.

Bernardino L, Balosso S, Ravizza T, Marchi N, Ku G, Randle JC, Malva JO, Vezzani A (2008) Inflammatory events in hippocampal slice cultures prime neuronal susceptibility to excitotoxic injury: a crucial role of $\mathrm{P} 2 \mathrm{X}_{7}$ receptor-mediated IL-1 $\beta$ release. J Neurochem 106:271-280 Available at: http://www.ncbi.nlm.nih.gov/pubmed/18384650 [Accessed January 2, 2019].

Bianco F, Perrotta C, Novellino L, Francolini M, Riganti L, Menna E, Saglietti L, Schuchman EH, Furlan R, Clementi E, Matteoli M, Verderio C (2009) Acid sphingomyelinase activity triggers microparticle release from glial cells. EMBO J 28:1043-1054 Available at: http://www.ncbi.nlm.nih.gov/pubmed/19300439 [Accessed January 5, 2019].

Bianco F, Pravettoni E, Colombo A, Schenk U, Möller T, Matteoli M, Verderio C (2005) 
Astrocyte-Derived ATP Induces Vesicle Shedding and IL-1 $\beta$ Release from Microglia. J Immunol 174:7268-7277 Available at: http://www.ncbi.nlm.nih.gov/pubmed/15905573 [Accessed January 5, 2019].

Binder LI, Frankfurter A, Rebhun LI (1985) The distribution of tau in the mammalian central nervous system. J Cell Biol 101:1371-1378 Available at: http://www.ncbi.nlm.nih.gov/pubmed/3930508 [Accessed January 1, 2019].

Bland CL, Byrne-Hoffman CN, Fernandez A, Rellick SL, Deng W, Klinke DJ (2018) Exosomes derived from B16F0 melanoma cells alter the transcriptome of cytotoxic T cells that impacts mitochondrial respiration. FEBS J 285:1033-1050 Available at: http://www.ncbi.nlm.nih.gov/pubmed/29399967 [Accessed November 16, 2018].

Blasko I, Veerhuis R, Stampfer-Kountchev M, Saurwein-Teissl M, Eikelenboom P, GrubeckLoebenstein B (2000) Costimulatory Effects of Interferon- $\gamma$ and Interleukin-1 $\beta$ or Tumor Necrosis Factor $\alpha$ on the Synthesis of $A \beta 1-40$ and $A \beta 1-42$ by Human Astrocytes. Neurobiol Dis 7:682-689 Available at: http://www.ncbi.nlm.nih.gov/pubmed/11114266 [Accessed November 1, 2018].

Blázquez G, Cañete T, Tobeña A, Giménez-Llort L, Fernández-Teruel A (2014) Cognitive and emotional profiles of aged Alzheimer's disease $(3 \times \mathrm{TgAD})$ mice: Effects of environmental enrichment and sexual dimorphism. Behav Brain Res 268:185-201 Available at: http://www.ncbi.nlm.nih.gov/pubmed/24746486 [Accessed August 12, 2017].

Bobrie A, Colombo M, Raposo G, Théry C (2011) Exosome Secretion: Molecular Mechanisms and Roles in Immune Responses. Traffic 12:1659-1668 Available at: http://www.ncbi.nlm.nih.gov/pubmed/21645191 [Accessed August 2, 2017].

Boldin MP, Taganov KD, Rao DS, Yang L, Zhao JL, Kalwani M, Garcia-Flores Y, Luong M, Devrekanli A, Xu J, Sun G, Tay J, Linsley PS, Baltimore D (2011) miR-146a is a significant brake on autoimmunity, myeloproliferation, and cancer in mice. J Exp Med 208:1189-1201 Available at: http://www.ncbi.nlm.nih.gov/pubmed/21555486 [Accessed August 23, 2018].

Bova IY, Bornstein NM, Korczyn AD (1996) Acute infection as a risk factor for ischemic stroke. Stroke 27:2204-2206 Available at: http://www.ncbi.nlm.nih.gov/pubmed/8969781 [Accessed December 16, 2018].

Braak H, Braak E (1991a) Neuropathological stageing of Alzheimer-related changes. Acta Neuropathol 82:239-259 Available at: http://www.ncbi.nlm.nih.gov/pubmed/1759558 [Accessed November 1, 2018].

Braak H, Braak E (1991b) Neuropathological stageing of Alzheimer-related changes. Acta Neuropathol 82:239-259 Available at: http://www.ncbi.nlm.nih.gov/pubmed/1759558 [Accessed August 9, 2018].

Bradley J (2008) TNF-mediated inflammatory disease. J Pathol 214:149-160 Available at: http://doi.wiley.com/10.1002/path.2287 [Accessed May 24, 2018].

Brier MR, Thomas JB, Snyder AZ, Benzinger TL, Zhang D, Raichle ME, Holtzman DM, Morris JC, Ances BM (2012) Loss of Intranetwork and Internetwork Resting State Functional Connections with Alzheimer's Disease Progression. J Neurosci 32:8890-8899 Available at: http://www.ncbi.nlm.nih.gov/pubmed/22745490 [Accessed August 21, 2018].

Broughton BRS, Reutens DC, Sobey CG (2009) Apoptotic Mechanisms After Cerebral Ischemia. Available at: http://stroke.ahajournals.org [Accessed January 7, 2019]. 
Buée L, Bussière T, Buée-Scherrer V, Delacourte A, Hof PR (2000) Tau protein isoforms, phosphorylation and role in neurodegenerative disorders. Brain Res Brain Res Rev 33:95130 Available at: http://www.ncbi.nlm.nih.gov/pubmed/10967355 [Accessed January 1, 2019].

Buttini M, Mir A, Appel K, Wiederhold KH, Limonta S, Gebicke-Haerter PJ, Boddeke HWGM (1997) Lipopolysaccharide induces expression of tumour necrosis factor alpha in rat brain: inhibition by methylprednisolone and by rolipram. Available at: http://europepmc.org/backend/ptpmcrender.fcgi?accid=PMC1565066\&blobtype=pdf [Accessed January 3, 2019].

Buzas EI, György B, Nagy G, Falus A, Gay S (2014) Emerging role of extracellular vesicles in inflammatory diseases. Nat Rev Rheumatol 10:356-364 Available at: http://www.nature.com/articles/nrrheum.2014.19 [Accessed January 6, 2019].

Caballero-Garrido E, Pena-Philippides JC, Lordkipanidze T, Bragin D, Yang Y, Erhardt EB, Roitbak T (2015) In Vivo Inhibition of miR-155 Promotes Recovery after Experimental Mouse Stroke. J Neurosci 35:12446-12464 Available at: http://www.ncbi.nlm.nih.gov/pubmed/26354913 [Accessed January 3, 2019].

Campbell LA, Rosenfeld ME (2015) Infection and Atherosclerosis Development. Arch Med Res 46:339-350 Available at: http://www.ncbi.nlm.nih.gov/pubmed/26004263 [Accessed January 3, 2019].

Cardoso AL, Guedes JR, de Lima MCP (2016) Role of microRNAs in the regulation of innate immune cells under neuroinflammatory conditions. Curr Opin Pharmacol 26:1-9 Available at: https://www.sciencedirect.com/science/article/pii/S1471489215001034?via\%3Dihub [Accessed August 21, 2018].

Cardoso AL, Guedes JR, Pereira de Almeida L, Pedroso de Lima MC (2012) miR-155 modulates microglia-mediated immune response by down-regulating SOCS-1 and promoting cytokine and nitric oxide production. Immunology 135:73-88 Available at: http://www.ncbi.nlm.nih.gov/pubmed/22043967 [Accessed January 6, 2019].

Carswell EA, Old LJ, Kassel RL, Green S, Fiore N, Williamson B (1975) An endotoxin-induced serum factor that causes necrosis of tumors. Proc Natl Acad Sci U S A 72:3666-3670 Available at: http://www.ncbi.nlm.nih.gov/pubmed/1103152 [Accessed May 24, 2018].

Castellani R, Hirai K, Aliev G, Drew KL, Nunomura A, Takeda A, Cash AD, Obrenovich ME, Perry G, Smith MA (2002) Role of mitochondrial dysfunction in Alzheimer's disease. J Neurosci Res 70:357-360 Available at: http://www.ncbi.nlm.nih.gov/pubmed/12391597 [Accessed January 7, 2019].

Cataldo AM, Broadwell RD (1986) Cytochemical identification of cerebral glycogen and glucose-6-phosphatase activity under normal and experimental conditions. II. Choroid plexus and ependymal epithelia, endothelia and pericytes. J Neurocytol 15:511-524 Available at: http://www.ncbi.nlm.nih.gov/pubmed/3018177 [Accessed January 5, 2019].

Cekanaviciute E, Buckwalter MS (2016) Astrocytes: Integrative Regulators of Neuroinflammation in Stroke and Other Neurological Diseases. Neurotherapeutics 13:685701 Available at: http://www.ncbi.nlm.nih.gov/pubmed/27677607 [Accessed January 20, 2019].

Cerutti C, Edwards LJ, de Vries HE, Sharrack B, Male DK, Romero IA (2017) MiR-126 and miR-126* regulate shear-resistant firm leukocyte adhesion to human brain endothelium. Sci 
Rep 7:45284 Available at: http://www.ncbi.nlm.nih.gov/pubmed/28358058 [Accessed August 13, 2017].

Cevik O, Baykal AT, Sener A (2016) Platelets Proteomic Profiles of Acute Ischemic Stroke Patients. PLoS One 11:e0158287 Available at: http://www.ncbi.nlm.nih.gov/pubmed/27336623 [Accessed January 6, 2019].

Cha DJ, Franklin JL, Dou Y, Liu Q, Higginbotham JN, Demory Beckler M, Weaver AM, Vickers K, Prasad N, Levy S, Zhang B, Coffey RJ, Patton JG (2015) KRAS-dependent sorting of miRNA to exosomes. Elife 4:e07197 Available at: http://www.ncbi.nlm.nih.gov/pubmed/26132860 [Accessed October 28, 2018].

Chaput N, Théry C (2011) Exosomes: immune properties and potential clinical implementations. Semin Immunopathol 33:419-440 Available at: http://www.ncbi.nlm.nih.gov/pubmed/21174094 [Accessed August 2, 2017].

Chaudhuri AD, Dastgheyb RM, Yoo S-W, Trout A, Talbot Jr CC, Hao H, Witwer KW, Haughey NJ (2018) TNF $\alpha$ and IL-1 $\beta$ modify the miRNA cargo of astrocyte shed extracellular vesicles to regulate neurotrophic signaling in neurons. Cell Death Dis 9:363 Available at: http://www.nature.com/articles/s41419-018-0369-4 [Accessed January 6, 2019].

Chen J, Cui C, Yang X, Xu J, Venkat P, Zacharek A, Yu P, Chopp M (2017a) MiR-126 Affects Brain-Heart Interaction after Cerebral Ischemic Stroke. Transl Stroke Res 8:374-385 Available at: http://www.ncbi.nlm.nih.gov/pubmed/28101763 [Accessed August 13, 2017]. Chen X, Jiang X-M, Zhao L-J, Sun L-L, Yan M-L, Tian Y, Zhang S, Duan M-J, Zhao H-M, Li W-R, Hao Y-Y, Wang L-B, Xiong Q-J, Ai J (2017b) MicroRNA-195 prevents dendritic degeneration and neuron death in rats following chronic brain hypoperfusion. Cell Death Dis 8 Available at: https://www.nature.com/cddis/journal/v8/n6/pdf/cddis2017243a.pdf [Accessed August 12, 2017].

Chevillet JR, Kang Q, Ruf IK, Briggs HA, Vojtech LN, Hughes SM, Cheng HH, Arroyo JD, Meredith EK, Gallichotte EN, Pogosova-Agadjanyan EL, Morrissey C, Stirewalt DL, Hladik F, Yu EY, Higano CS, Tewari M (2014) Quantitative and stoichiometric analysis of the microRNA content of exosomes. Proc Natl Acad Sci 111:14888-14893 Available at: http://www.ncbi.nlm.nih.gov/pubmed/25267620 [Accessed November 24, 2018].

Chipuk JE, Green DR (2008) How do BCL-2 proteins induce mitochondrial outer membrane permeabilization? Trends Cell Biol 18:157-164 Available at: http://www.ncbi.nlm.nih.gov/pubmed/18314333 [Accessed January 7, 2019].

Cho KJ, Song J, Oh Y, Lee JE (2015) MicroRNA-Let-7a regulates the function of microglia in inflammation. Mol Cell Neurosci 68:167-176 Available at: http://linkinghub.elsevier.com/retrieve/pii/S1044743115300051 [Accessed August 3, 2017].

Cogswell JP, Ward J, Taylor IA, Waters M, Shi Y, Cannon B, Kelnar K, Kemppainen J, Brown D, Chen C, Prinjha RK, Richardson JC, Saunders AM, Roses AD, Richards CA (2008) Identification of miRNA changes in Alzheimer's disease brain and CSF yields putative biomarkers and insights into disease pathways. J Alzheimers Dis 14:27-41 Available at: http://www.ncbi.nlm.nih.gov/pubmed/18525125 [Accessed August 23, 2018].

Collins LV, Hajizadeh S, Holme E, Jonsson I-M, Tarkowski A (2004) Endogenously oxidized mitochondrial DNA induces in vivo and in vitro inflammatory responses. J Leukoc Biol 75:995-1000 Available at: http://www.ncbi.nlm.nih.gov/pubmed/14982943 [Accessed January 2, 2019]. 
Colombo M, Raposo G, Théry C (2014) Biogenesis, Secretion, and Intercellular Interactions of Exosomes and Other Extracellular Vesicles. Annu Rev Cell Dev Biol 30:255-289 Available at: file:///home/chronos/u-

9f9ac28c290b0e5538196eabebee0230bde01893/Downloads/annurev-cellbio-101512122326.pdf [Accessed July 7, 2017].

Corda S, Laplace C, Vicaut E, Duranteau J (2001) Rapid Reactive Oxygen Species Production by Mitochondria in Endothelial Cells Exposed to Tumor Necrosis Factor- $\alpha$ Is Mediated by Ceramide. Am J Respir Cell Mol Biol 24:762-768 Available at: http://www.ncbi.nlm.nih.gov/pubmed/11415943 [Accessed January 7, 2019].

Couch Y, Akbar N, Davis S, Fischer R, Dickens AM, Neuhaus AA, Burgess AI, Rothwell PM, Buchan AM (2017a) Inflammatory Stroke Extracellular Vesicles Induce Macrophage Activation. Stroke 48:2292-2296 Available at: http://www.ncbi.nlm.nih.gov/pubmed/28536169 [Accessed December 16, 2018].

Couch Y, Akbar N, Roodselaar J, Evans MC, Gardiner C, Sargent I, Romero IA, Bristow A, Buchan AM, Haughey N, Anthony DC (2017b) Circulating endothelial cell-derived extracellular vesicles mediate the acute phase response and sickness behaviour associated with CNS inflammation. Sci Rep 7:9574 Available at: http://www.nature.com/articles/s41598-017-09710-3 [Accessed December 16, 2018].

Crain JM, Nikodemova M, Watters JJ (2013) Microglia express distinct M1 and M2 phenotypic markers in the postnatal and adult central nervous system in male and female mice. $\mathrm{J}$ Neurosci Res 91:1143-1151 Available at: http://www.ncbi.nlm.nih.gov/pubmed/23686747 [Accessed January 1, 2019].

Cui JG, Li YY, Zhao Y, Bhattacharjee S, Lukiw WJ (2010) Differential Regulation of Interleukin-1 Receptor-associated Kinase-1 (IRAK-1) and IRAK-2 by MicroRNA-146a and NF- $\kappa$ B in Stressed Human Astroglial Cells and in Alzheimer Disease. J Biol Chem 285:38951-38960 Available at: http://www.ncbi.nlm.nih.gov/pubmed/20937840 [Accessed August 21, 2018].

D'Adamo S, Alvarez-Garcia O, Muramatsu Y, Flamigni F, Lotz MK (2016) MicroRNA-155 suppresses autophagy in chondrocytes by modulating expression of autophagy proteins. Osteoarthr Cartil 24:1082-1091 Available at: http://www.ncbi.nlm.nih.gov/pubmed/26805019 [Accessed January 3, 2019].

Dalpke A, Heeg K, Bartz H, Baetz A (2008) Regulation of innate immunity by suppressor of cytokine signaling (SOCS) proteins. Immunobiology 213:225-235 Available at: http://www.ncbi.nlm.nih.gov/pubmed/18406369 [Accessed August 4, 2017].

Dasgupta N, Peng Y, Tan Z, Ciraolo G, Wang D, Li R (2015) miRNAs in mtDNA-less cell mitochondria. Cell Death Discov 1:15004 Available at: http://www.nature.com/articles/cddiscovery20154 [Accessed November 16, 2018].

Davalos D, Grutzendler J, Yang G, Kim J V, Zuo Y, Jung S, Littman DR, Dustin ML, Gan W-B (2005) ATP mediates rapid microglial response to local brain injury in vivo. Nat Neurosci 8:752-758 Available at: http://www.ncbi.nlm.nih.gov/pubmed/15895084 [Accessed August 4, 2017].

de Leon MJ, Convit A, Wolf OT, Tarshish CY, DeSanti S, Rusinek H, Tsui W, Kandil E, Scherer AJ, Roche A, Imossi A, Thorn E, Bobinski M, Caraos C, Lesbre P, Schlyer D, Poirier J, Reisberg B, Fowler J (2001) Prediction of cognitive decline in normal elderly 
subjects with 2-[18F]fluoro-2-deoxy-D-glucose/positron-emission tomography (FDG/PET). Proc Natl Acad Sci 98:10966-10971 Available at: http://www.ncbi.nlm.nih.gov/pubmed/11526211 [Accessed August 9, 2018].

Decourt B, Lahiri D, Sabbagh M (2016) Targeting Tumor Necrosis Factor Alpha for Alzheimer's Disease. Curr Alzheimer Res 13:1-1 Available at: http://www.eurekaselect.com/openurl/content.php?genre=article\&doi=10.2174/1567205013 666160930110551 [Accessed November 25, 2018].

Denk J, Boelmans K, Siegismund C, Lassner D, Arlt S, Jahn H (2015) MicroRNA Profiling of CSF Reveals Potential Biomarkers to Detect Alzheimer`s Disease. PLoS One 10:e0126423 Available at: http://www.ncbi.nlm.nih.gov/pubmed/25992776 [Accessed August 23, 2018].

Di Rocco G, Baldari S, Toietta G (2016) Towards Therapeutic Delivery of Extracellular Vesicles: Strategies for In Vivo Tracking and Biodistribution Analysis. Stem Cells Int 2016:1-12 Available at: https://www.hindawi.com/journals/sci/2016/5029619/ [Accessed January 6, 2019].

Ding W-X, Yin X-M (2012) Mitophagy: mechanisms, pathophysiological roles, and analysis. Biol Chem 393:547-564 Available at: http://www.ncbi.nlm.nih.gov/pubmed/22944659 [Accessed January 6, 2019].

Djavaheri-Mergny M, Amelotti M, Mathieu J, Besançon F, Bauvy C, Souquère S, Pierron G, Codogno P (2006) NF-kappaB activation represses tumor necrosis factor-alpha-induced autophagy. J Biol Chem 281:30373-30382 Available at: http://www.ncbi.nlm.nih.gov/pubmed/16857678 [Accessed January 3, 2019].

Doench JG, Sharp PA (2004) Specificity of microRNA target selection in translational repression. Genes Dev 18:504-511 Available at: http://www.ncbi.nlm.nih.gov/pubmed/15014042 [Accessed January 2, 2019].

Doll DN, Engler-Chiurazzi EB, Lewis SE, Hu H, Kerr AE, Ren X, Simpkins JW (2015a) Lipopolysaccharide exacerbates infarct size and results in worsened post-stroke behavioral outcomes. Behav Brain Funct 11:32 Available at: http://www.ncbi.nlm.nih.gov/pubmed/26463864 [Accessed January 4, 2019].

Doll DN, Hu H, Sun J, Lewis SE, Simpkins JW, Ren X (2015b) Mitochondrial Crisis in Cerebrovascular Endothelial Cells Opens the Blood-Brain Barrier. Stroke 46:1681-1689 Available at: http://www.ncbi.nlm.nih.gov/pubmed/25922503 [Accessed December 12, 2018].

Dong H, Li J, Huang L, Chen X, Li D, Wang T, Hu C, Xu J, Zhang C, Zen K, Xiao S, Yan Q, Wang C, Zhang C-Y (2015) Serum MicroRNA Profiles Serve as Novel Biomarkers for the Diagnosis of Alzheimer's Disease. Dis Markers 2015:625659 Available at: http://www.ncbi.nlm.nih.gov/pubmed/26078483 [Accessed August 23, 2018].

Dong Y, Benveniste EN (2001) Immune function of astrocytes. Glia 36:180-190 Available at: http://www.ncbi.nlm.nih.gov/pubmed/11596126 [Accessed August 24, 2018].

Dozio V, Sanchez J-C (2017) Characterisation of extracellular vesicle-subsets derived from brain endothelial cells and analysis of their protein cargo modulation after TNF exposure. $\mathbf{J}$ Extracell vesicles 6:1302705 Available at: http://www.ncbi.nlm.nih.gov/pubmed/28473883 [Accessed January 6, 2019].

Eikelenboom P, Hack CE, Rozemuller JM, Stam FC (1989) Complement activation in amyloid plaques in Alzheimer's dementia. Virchows Arch B Cell Pathol Incl Mol Pathol 56:259- 
262 Available at: http://www.ncbi.nlm.nih.gov/pubmed/2565620 [Accessed August 12, 2017].

Eikelenboom P, Veerhuis R, Scheper W, Rozemuller AJM, van Gool WA, Hoozemans JJM (2006) The significance of neuroinflammation in understanding Alzheimer's disease. J Neural Transm 113:1685-1695 Available at: http://www.ncbi.nlm.nih.gov/pubmed/17036175 [Accessed August 12, 2017].

Elkind MS V, Carty CL, O’Meara ES, Lumley T, Lefkowitz D, Kronmal RA, Longstreth WT, Jr. (2011) Hospitalization for infection and risk of acute ischemic stroke: the Cardiovascular Health Study. Stroke 42:1851-1856 Available at: http://www.ncbi.nlm.nih.gov/pubmed/21546476 [Accessed December 16, 2018].

Erikssen J, Hellem A, Stormorken H (1977) Chronic effect of smoking on platelet count and \&quot;platelet adhesiveness\&quot; in presumably healthy middle-aged men. Thromb Haemost 38:606-611 Available at: http://www.ncbi.nlm.nih.gov/pubmed/579506 [Accessed January 6, 2019].

Erne P, Resink TJ, Bürgisser E, Bühler FR (1985) Platelets and hypertension. J Cardiovasc Pharmacol 7 Suppl 6:S103-8 Available at: http://www.ncbi.nlm.nih.gov/pubmed/2414569 [Accessed January 6, 2019].

Falcão AS, Carvalho LAR, Lidónio G, Vaz AR, Lucas SD, Moreira R, Brites D (2017) Dipeptidyl Vinyl Sulfone as a Novel Chemical Tool to Inhibit HMGB1/NLRP3Inflammasome and Inflamma-miRs in A $\beta$-Mediated Microglial Inflammation. ACS Chem Neurosci 8:89-99 Available at: http://www.ncbi.nlm.nih.gov/pubmed/27797173 [Accessed August 12, 2017].

Falkowska A, Gutowska I, Goschorska M, Nowacki P, Chlubek D, Baranowska-Bosiacka I (2015) Energy Metabolism of the Brain, Including the Cooperation between Astrocytes and Neurons, Especially in the Context of Glycogen Metabolism. Int J Mol Sci 16:25959-25981 Available at: http://www.ncbi.nlm.nih.gov/pubmed/26528968 [Accessed January 5, 2019].

Fan C, Wu Q, Ye X, Luo H, Yan D, Xiong Y, Zhu H, Diao Y, Zhang W, Wan J (2016) Role of miR-211 in Neuronal Differentiation and Viability: Implications to Pathogenesis of Alzheimer's Disease. Front Aging Neurosci 8:166 Available at: http://www.ncbi.nlm.nih.gov/pubmed/27458373 [Accessed August 12, 2017].

Fan Y, Ding S, Sun Y, Zhao B, Pan Y, Wan J (2017) MiR-377 regulates inflammation and angiogenesis in rats after cerebral ischemic injury. J Cell Biochem Available at: http://www.ncbi.nlm.nih.gov/pubmed/28569430 [Accessed August 13, 2017].

Fauré J, Lachenal G, Court M, Hirrlinger J, Chatellard-Causse C, Blot B, Grange J, Schoehn G, Goldberg Y, Boyer V, Kirchhoff F, Raposo G, Garin J, Sadoul R (2006) Exosomes are released by cultured cortical neurones. Mol Cell Neurosci 31:642-648 Available at: https://www.sciencedirect.com/science/article/pii/S1044743105003027 [Accessed January 5, 2019].

Fevrier B, Vilette D, Laude H, Raposo G (2005) Exosomes: A Bubble Ride for Prions? Traffic 6:10-17 Available at: http://www.ncbi.nlm.nih.gov/pubmed/15569241 [Accessed January $5,2019]$.

Fillit H, Ding WH, Buee L, Kalman J, Altstiel L, Lawlor B, Wolf-Klein G (1991) Elevated circulating tumor necrosis factor levels in Alzheimer's disease. Neurosci Lett 129:318-320 Available at: http://www.ncbi.nlm.nih.gov/pubmed/1745413 [Accessed November 1, 2018]. 
Fouquet M, Desgranges B, Landeau B, Duchesnay E, Mézenge F, de la Sayette V, Viader F, Baron J-C, Eustache F, Chételat G (2009) Longitudinal brain metabolic changes from amnestic mild cognitive impairment to Alzheimer's disease. Brain 132:2058-2067 Available at: http://www.ncbi.nlm.nih.gov/pubmed/19477964 [Accessed January 7, 2019].

Frackowiak J, Wisniewski HM, Wegiel J, Merz GS, Iqbal K, Wang KC (1992) Ultrastructure of the microglia that phagocytose amyloid and the microglia that produce beta-amyloid fibrils. Acta Neuropathol 84:225-233 Available at: http://www.ncbi.nlm.nih.gov/pubmed/1414275 [Accessed August 12, 2017].

Franceschi C, Garagnani P, Parini P, Giuliani C, Santoro A (2018) Inflammaging: a new immune-metabolic viewpoint for age-related diseases. Nat Rev Endocrinol 14:576-590 Available at: http://www.ncbi.nlm.nih.gov/pubmed/30046148 [Accessed January 1, 2019].

Frühbeis C, Fröhlich D, Kuo WP, Amphornrat J, Thilemann S, Saab AS, Kirchhoff F, Möbius W, Goebbels S, Nave K-A, Schneider A, Simons M, Klugmann M, Trotter J, KrämerAlbers E-M (2013) Neurotransmitter-Triggered Transfer of Exosomes Mediates Oligodendrocyte-Neuron Communication Barres BA, ed. PLoS Biol 11:e1001604 Available at: https://dx.plos.org/10.1371/journal.pbio.1001604 [Accessed January 5, 2019].

Fu H, Liu B, Frost JL, Hong S, Jin M, Ostaszewski B, Shankar GM, Costantino IM, Carroll MC, Mayadas TN, Lemere CA (2012) Complement component C3 and complement receptor type 3 contribute to the phagocytosis and clearance of fibrillar A $\beta$ by microglia. Glia 60:993-1003 Available at: http://www.ncbi.nlm.nih.gov/pubmed/22438044 [Accessed August 12, 2017].

Fu Y, Zhang L, Zhang F, Tang T, Zhou Q, Feng C, Jin Y, Wu Z (2017) Exosome-mediated miR146a transfer suppresses type I interferon response and facilitates EV71 infection Coyne CB, ed. PLOS Pathog 13:e1006611 Available at: http://www.ncbi.nlm.nih.gov/pubmed/28910400 [Accessed August 23, 2018].

Gabuzda D, Busciglio J, Chen LB, Matsudaira P, Yankner BA (1994) Inhibition of energy metabolism alters the processing of amyloid precursor protein and induces a potentially amyloidogenic derivative. J Biol Chem 269:13623-13628 Available at: http://www.ncbi.nlm.nih.gov/pubmed/8175797 [Accessed January 7, 2019].

Galea I, Bechmann I, Perry VH (2007) What is immune privilege (not)? Trends Immunol 28:1218.

Gavillet M, Allaman I, Magistretti PJ (2008) Modulation of astrocytic metabolic phenotype by proinflammatory cytokines. Glia 56:975-989 Available at: http://www.ncbi.nlm.nih.gov/pubmed/18383346 [Accessed August 10, 2018].

Gehrmann J, Matsumoto Y, Kreutzberg GW (1995) Microglia: intrinsic immuneffector cell of the brain. Brain Res Brain Res Rev 20:269-287 Available at: http://www.ncbi.nlm.nih.gov/pubmed/7550361 [Accessed August 4, 2017].

Gemmell CH, Sefton M V, Yeo EL (1993) Platelet-derived microparticle formation involves glycoprotein IIb-IIIa. Inhibition by RGDS and a Glanzmann's thrombasthenia defect. J Biol Chem 268:14586-14589 Available at: http://www.ncbi.nlm.nih.gov/pubmed/8325838 [Accessed January 6, 2019].

Ghahremanfard F, Semnani V, Ghorbani R, Malek F, Behzadfar A, Zahmatkesh M (2015) Effects of cigarette smoking on morphological features of platelets in healthy men. Saudi Med J 36:847-850 Available at: http://www.ncbi.nlm.nih.gov/pubmed/26108590 [Accessed 
January 6, 2019].

Glebov K, Löchner M, Jabs R, Lau T, Merkel O, Schloss P, Steinhäuser C, Walter J (2015)

Serotonin stimulates secretion of exosomes from microglia cells. Glia 63:626-634

Available at: http://www.ncbi.nlm.nih.gov/pubmed/25451814 [Accessed October 30, 2018].

Goetzl EJ, Mustapic M, Kapogiannis D, Eitan E, Lobach I V., Goetzl L, Schwartz JB, Miller BL (2016) Cargo proteins of plasma astrocyte-derived exosomes in Alzheimer's disease.

FASEB J 30:3853-3859 Available at: http://www.fasebj.org/doi/10.1096/fj.201600756R [Accessed January 5, 2019].

Goetzl EJ, Schwartz JB, Abner EL, Jicha GA, Kapogiannis D (2018) High complement levels in astrocyte-derived exosomes of Alzheimer disease. Ann Neurol 83:544-552 Available at: http://www.ncbi.nlm.nih.gov/pubmed/29406582 [Accessed January 5, 2019].

Golan H, Levav T, Mendelsohn A, Huleihel M (2004) Involvement of tumor necrosis factor alpha in hippocampal development and function. Cereb Cortex 14:97-105 Available at: http://www.ncbi.nlm.nih.gov/pubmed/14654461 [Accessed January 2, 2019].

Goldberg MP, Choi DW (1993) Combined oxygen and glucose deprivation in cortical cell culture: calcium-dependent and calcium-independent mechanisms of neuronal injury. J Neurosci 13:3510-3524 Available at: http://www.ncbi.nlm.nih.gov/pubmed/8101871 [Accessed August 24, 2018].

González H, Elgueta D, Montoya A, Pacheco R (2014) Neuroimmune regulation of microglial activity involved in neuroinflammation and neurodegenerative diseases. J Neuroimmunol 274:1-13 Available at: http://www.ncbi.nlm.nih.gov/pubmed/25091432 [Accessed August 12, 2017].

Grau AJ, Buggle F, Heindl S, Steichen-Wiehn C, Banerjee T, Maiwald M, Rohlfs M, Suhr H, Fiehn W, Becher H (1995) Recent infection as a risk factor for cerebrovascular ischemia. Stroke 26:373-379 Available at: http://www.ncbi.nlm.nih.gov/pubmed/7886709 [Accessed December 16, 2018].

Grau AJ, Fischer B, Barth C, Ling P, Lichy C, Buggle F (2005) Influenza Vaccination Is Associated With a Reduced Risk of Stroke. Stroke 36:1501-1506 Available at: http://www.ncbi.nlm.nih.gov/pubmed/15947266 [Accessed December 16, 2018].

Greco V, Hannus M, Eaton S (2001) Argosomes: A Potential Vehicle for the Spread of Morphogens through Epithelia. Cell 106:633-645 Available at: https://www.sciencedirect.com/science/article/pii/S0092867401004846 [Accessed January $5,2019]$.

Guan R, Zou W, Dai X, Yu X, Liu H, Chen Q, Teng W (2018) Mitophagy, a potential therapeutic target for stroke. J Biomed Sci 25:87 Available at: http://www.ncbi.nlm.nih.gov/pubmed/30501621 [Accessed January 6, 2019].

Guedes J, Cardoso ALC, Pedroso de Lima MC (2013) Involvement of microRNA in microgliamediated immune response. Clin Dev Immunol 2013:186872 Available at: http://www.ncbi.nlm.nih.gov/pubmed/23762086 [Accessed August 3, 2017]

Guedes PMM, de Andrade CM, Nunes DF, de Sena Pereira N, Queiroga TBD, Machado-Coelho GLL, Nascimento MSL, Do-Valle-Matta MA, da Câmara ACJ, Chiari E, Galvão LM da C (2016) Inflammation Enhances the Risks of Stroke and Death in Chronic Chagas Disease Patients. PLoS Negl Trop Dis 10:e0004669 Available at: http://www.ncbi.nlm.nih.gov/pubmed/27115869 [Accessed December 16, 2018]. 
Guez-Barber D, Fanous S, Harvey BK, Zhang Y, Lehrmann E, Becker KG, Picciotto MR, Hope BT (2012) FACS purification of immunolabeled cell types from adult rat brain. J Neurosci Methods 203:10-18 Available at: http://www.ncbi.nlm.nih.gov/pubmed/21911005 [Accessed January 6, 2019].

Gupta SC, Hevia D, Patchva S, Park B, Koh W, Aggarwal BB (2012) Upsides and downsides of reactive oxygen species for cancer: the roles of reactive oxygen species in tumorigenesis, prevention, and therapy. Antioxid Redox Signal 16:1295-1322 Available at: http://www.ncbi.nlm.nih.gov/pubmed/22117137 [Accessed January 2, 2019].

Guzy RD, Hoyos B, Robin E, Chen H, Liu L, Mansfield KD, Simon MC, Hammerling U, Schumacker PT (2005) Mitochondrial complex III is required for hypoxia-induced ROS production and cellular oxygen sensing. Cell Metab 1:401-408 Available at: http://www.ncbi.nlm.nih.gov/pubmed/16054089 [Accessed January 7, 2019].

Ha M, Kim VN (2014) Regulation of microRNA biogenesis. Nat Rev Mol Cell Biol 15:509-524 Available at: http://www.ncbi.nlm.nih.gov/pubmed/25027649 [Accessed January 2, 2019].

Haddad JJ, Saadé NE, Safieh-Garabedian B (2002) Cytokines and neuro-immune-endocrine interactions: a role for the hypothalamic-pituitary-adrenal revolving axis. J Neuroimmunol 133:1-19 Available at: http://www.ncbi.nlm.nih.gov/pubmed/12446003 [Accessed January 3, 2019].

Hammond SM (2015) An overview of microRNAs. Adv Drug Deliv Rev 87:3-14 Available at: http://www.ncbi.nlm.nih.gov/pubmed/25979468 [Accessed January 7, 2019].

Hanisch U-K (2002) Microglia as a source and target of cytokines. Glia 40:140-155 Available at: http://doi.wiley.com/10.1002/glia.10161 [Accessed November 1, 2018].

Hara H, Friedlander RM, Gagliardini V, Ayata C, Fink K, Huang Z, Shimizu-Sasamata M, Yuan J, Moskowitz MA, Fiskum G, Reed JC (1997) Inhibition of interleukin 1 converting enzyme family proteases reduces ischemic and excitotoxic neuronal damage. Proc Natl Acad Sci 94:2007-2012 Available at: http://www.ncbi.nlm.nih.gov/pubmed/9050895 [Accessed January 6, 2019].

Harding C, Heuser J, Stahl P (1983) Receptor-mediated endocytosis of transferrin and recycling of the transferrin receptor in rat reticulocytes. J Cell Biol 97:329-339 Available at: http://www.ncbi.nlm.nih.gov/pubmed/6309857 [Accessed May 22, 2018].

Harding C, Heuser J, Stahl P (1984) Endocytosis and intracellular processing of transferrin and colloidal gold-transferrin in rat reticulocytes: demonstration of a pathway for receptor shedding. Eur J Cell Biol 35:256-263 Available at: http://www.ncbi.nlm.nih.gov/pubmed/6151502 [Accessed January 5, 2019].

Harding C, Stahl P (1983) Transferrin recycling in reticulocytes: $\mathrm{pH}$ and iron are important determinants of ligand binding and processing. Biochem Biophys Res Commun 113:650658 Available at: http://www.ncbi.nlm.nih.gov/pubmed/6870878 [Accessed January 5, 2019].

Harting MT, Srivastava AK, Zhaorigetu S, Bair H, Prabhakara KS, Toledano Furman NE, Vykoukal J V., Ruppert KA, Cox CS, Olson SD (2018) Inflammation-Stimulated Mesenchymal Stromal Cell-Derived Extracellular Vesicles Attenuate Inflammation. Stem Cells 36:79-90 Available at: http://www.ncbi.nlm.nih.gov/pubmed/29076623 [Accessed October 29, 2018].

Heldmann U, Thored P, Claasen J-H, Arvidsson A, Kokaia Z, Lindvall O (2005) TNF-alpha 
antibody infusion impairs survival of stroke-generated neuroblasts in adult rat brain. Exp Neurol 196:204-208 Available at: http://www.ncbi.nlm.nih.gov/pubmed/16157335

[Accessed January 2, 2019].

Herber DL, Mercer M, Roth LM, Symmonds K, Maloney J, Wilson N, Freeman MJ, Morgan D, Gordon MN (2007) Microglial Activation is Required for A $\beta$ Clearance After Intracranial Injection of Lipopolysaccharide in APP Transgenic Mice. J Neuroimmune Pharmacol 2:222-231 Available at: http://www.ncbi.nlm.nih.gov/pubmed/18040847 [Accessed August 12, 2017].

Herber DL, Roth LM, Wilson D, Wilson N, Mason JE, Morgan D, Gordon MN (2004) Timedependent reduction in A $\beta$ levels after intracranial LPS administration in APP transgenic mice. Exp Neurol 190:245-253 Available at: http://www.ncbi.nlm.nih.gov/pubmed/15473997 [Accessed August 12, 2017].

Hickey WF, Hsu BL, Kimura H (1991) T-lymphocyte entry into the central nervous system. J Neurosci Res 28:254-260 Available at: http://www.ncbi.nlm.nih.gov/pubmed/2033653 [Accessed August 3, 2017].

Hill BG, Dranka BP, Zou L, Chatham JC, Darley-Usmar VM (2009) Importance of the bioenergetic reserve capacity in response to cardiomyocyte stress induced by 4hydroxynonenal. Biochem J 424:99-107 Available at: http://www.ncbi.nlm.nih.gov/pubmed/19740075 [Accessed November 28, 2018]. Hinger SA, Cha DJ, Franklin JL, Higginbotham JN, Dou Y, Ping J, Shu L, Prasad N, Levy S, Zhang B, Liu Q, Weaver AM, Coffey RJ, Patton JG (2018) Diverse Long RNAs Are Differentially Sorted into Extracellular Vesicles Secreted by Colorectal Cancer Cells. Cell Rep 25:715-725.e4 Available at: https://www.sciencedirect.com/science/article/pii/S2211124718314992 [Accessed October 28, 2018].

Hong S, Dissing-Olesen L, Stevens B (2016) New insights on the role of microglia in synaptic pruning in health and disease. Curr Opin Neurobiol 36:128-134 Available at: http://www.ncbi.nlm.nih.gov/pubmed/26745839 [Accessed August 12, 2017].

Hough KP, Trevor JL, Strenkowski JG, Wang Y, Chacko BK, Tousif S, Chanda D, Steele C, Antony VB, Dokland T, Ouyang X, Zhang J, Duncan SR, Thannickal VJ, Darley-Usmar VM, Deshane JS (2018) Exosomal transfer of mitochondria from airway myeloid-derived regulatory cells to T cells. Redox Biol 18:54-64 Available at: https://www.sciencedirect.com/science/article/pii/S2213231718304701 [Accessed November 14, 2018].

Huntzinger E, Izaurralde E (2011) Gene silencing by microRNAs: contributions of translational repression and mRNA decay. Nat Rev Genet 12:99-110 Available at: http://www.ncbi.nlm.nih.gov/pubmed/21245828 [Accessed November 19, 2018].

Iwasaki S, Kawamata T, Tomari Y (2009) Drosophila Argonaute1 and Argonaute2 Employ Distinct Mechanisms for Translational Repression. Mol Cell 34:58-67 Available at: http://www.ncbi.nlm.nih.gov/pubmed/19268617 [Accessed January 2, 2019].

Iyer A, Zurolo E, Prabowo A, Fluiter K, Spliet WGM, van Rijen PC, Gorter JA, Aronica E (2012) MicroRNA-146a: A Key Regulator of Astrocyte-Mediated Inflammatory Response Villoslada P, ed. PLoS One 7:e44789 Available at: http://www.ncbi.nlm.nih.gov/pubmed/23028621 [Accessed August 21, 2018]. 
Janowska-Wieczorek A, Wysoczynski M, Kijowski J, Marquez-Curtis L, Machalinski B, Ratajczak J, Ratajczak MZ (2005) Microvesicles derived from activated platelets induce metastasis and angiogenesis in lung cancer. Int J Cancer 113:752-760 Available at: http://www.ncbi.nlm.nih.gov/pubmed/15499615 [Accessed May 29, 2018].

Jastroch M, Divakaruni AS, Mookerjee S, Treberg JR, Brand MD (2010) Mitochondrial proton and electron leaks. Essays Biochem 47:53-67 Available at: http://www.ncbi.nlm.nih.gov/pubmed/20533900 [Accessed November 1, 2018].

Johnson AA, Akman K, Calimport SRG, Wuttke D, Stolzing A, de Magalhães JP (2012) The role of DNA methylation in aging, rejuvenation, and age-related disease. Rejuvenation Res 15:483-494 Available at: http://www.ncbi.nlm.nih.gov/pubmed/23098078 [Accessed January 3, 2019].

Johnstone RM, Adam M, Hammond JR, Orr L, Turbide C (1987) Vesicle formation during reticulocyte maturation. Association of plasma membrane activities with released vesicles (exosomes). J Biol Chem 262:9412-9420.

Jovičić A, Gitler AD (2017) Distinct repertoires of microRNAs present in mouse astrocytes compared to astrocyte-secreted exosomes Dehghani F, ed. PLoS One 12:e0171418 Available at: https://dx.plos.org/10.1371/journal.pone.0171418 [Accessed January 5, 2019].

Kabe Y, Ando K, Hirao S, Yoshida M, Handa H (2005) Redox Regulation of NF-кB Activation: Distinct Redox Regulation Between the Cytoplasm and the Nucleus. Antioxid Redox Signal 7:395-403 Available at: http://www.liebertpub.com/doi/10.1089/ars.2005.7.395 [Accessed January 2, 2019].

Kadavath H, Hofele R V., Biernat J, Kumar S, Tepper K, Urlaub H, Mandelkow E, Zweckstetter M (2015) Tau stabilizes microtubules by binding at the interface between tubulin heterodimers. Proc Natl Acad Sci 112:7501-7506 Available at: http://www.ncbi.nlm.nih.gov/pubmed/26034266 [Accessed January 1, 2019].

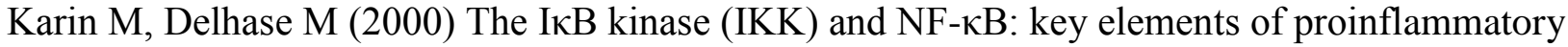
signalling. Semin Immunol 12:85-98 Available at: https://www.sciencedirect.com/science/article/pii/S1044532300902100?via\%3Dihub [Accessed January 2, 2019].

Kawai T, Akira S (2010) The role of pattern-recognition receptors in innate immunity: update on Toll-like receptors. Nat Immunol 11:373-384 Available at: http://www.nature.com/articles/ni.1863 [Accessed May 24, 2018].

Kawasaki T, Kawai T (2014) Toll-Like Receptor Signaling Pathways. Front Immunol 5:461 Available at: http://www.ncbi.nlm.nih.gov/pubmed/25309543 [Accessed May 23, 2018].

Keohane A, Ryan S, Maloney E, Sullivan AM, Nolan YM (2010) Tumour necrosis factor- $\alpha$ impairs neuronal differentiation but not proliferation of hippocampal neural precursor cells: Role of Hes1. Mol Cell Neurosci 43:127-135 Available at: http://www.ncbi.nlm.nih.gov/pubmed/19840854 [Accessed January 2, 2019].

Khangura RK, Bali A, Jaggi AS, Singh N (2017) Histone acetylation and histone deacetylation in neuropathic pain: An unresolved puzzle? Eur J Pharmacol 795:36-42 Available at: http://www.ncbi.nlm.nih.gov/pubmed/27916557 [Accessed January 20, 2019].

Koch S, Gonzalez N (2013) Preconditioning the Human Brain. Stroke 44:1748-1753 Available at: https://www.ahajournals.org/doi/10.1161/STROKEAHA.111.000773 [Accessed January 8, 2019]. 
Koellhoffer E, McCullough L, Ritzel R (2017) Old Maids: Aging and Its Impact on Microglia Function. Int J Mol Sci 18:769 Available at: http://www.ncbi.nlm.nih.gov/pubmed/28379162 [Accessed January 1, 2019].

Kopec KK, Carroll RT (1998) Alzheimer's beta-amyloid peptide 1-42 induces a phagocytic response in murine microglia. J Neurochem 71:2123-2131 Available at: http://www.ncbi.nlm.nih.gov/pubmed/9798938 [Accessed August 12, 2017].

Krabbe G, Halle A, Matyash V, Rinnenthal JL, Eom GD, Bernhardt U, Miller KR, Prokop S, Kettenmann H, Heppner FL (2013) Functional Impairment of Microglia Coincides with Beta-Amyloid Deposition in Mice with Alzheimer-Like Pathology Priller J, ed. PLoS One 8:e60921 Available at: http://dx.plos.org/10.1371/journal.pone.0060921 [Accessed August $9,2018]$.

Krämer-Albers E-M (2017) Ticket to Ride: Targeting Proteins to Exosomes for Brain Delivery. Mol Ther 25:1264-1266 Available at: http://www.ncbi.nlm.nih.gov/pubmed/28499750 [Accessed January 6, 2019].

Krämer-Albers E-M, Bretz N, Tenzer S, Winterstein C, Möbius W, Berger H, Nave K-A, Schild $\mathrm{H}$, Trotter J (2007) Oligodendrocytes secrete exosomes containing major myelin and stressprotective proteins: Trophic support for axons? PROTEOMICS - Clin Appl 1:1446-1461 Available at: http://www.ncbi.nlm.nih.gov/pubmed/21136642 [Accessed January 5, 2019].

Krek A, Grün D, Poy MN, Wolf R, Rosenberg L, Epstein EJ, MacMenamin P, da Piedade I, Gunsalus KC, Stoffel M, Rajewsky N (2005) Combinatorial microRNA target predictions. Nat Genet 37:495-500 Available at: http://www.ncbi.nlm.nih.gov/pubmed/15806104 [Accessed November 19, 2018].

Krupinski J, Carrera C, Muiño E, Torres N, Al-Baradie R, Cullell N, Fernandez-Cadenas I (2018) DNA Methylation in Stroke. Update of Latest Advances. Comput Struct Biotechnol J 16:1-5 Available at: http://www.ncbi.nlm.nih.gov/pubmed/29321829 [Accessed January 3, 2019].

Kumar S, Reddy PH (2016) Are circulating microRNAs peripheral biomarkers for Alzheimer's disease? Biochim Biophys Acta - Mol Basis Dis 1862:1617-1627 Available at: http://linkinghub.elsevier.com/retrieve/pii/S0925443916301429 [Accessed August 19, 2017].

Lafourcade C, Ramírez JP, Luarte A, Fernández A, Wyneken U (2016) MIRNAS in AstrocyteDerived Exosomes as Possible Mediators of Neuronal Plasticity. J Exp Neurosci 10s1:JEN.S39916 Available at: http://journals.sagepub.com/doi/10.4137/JEN.S39916 [Accessed January 5, 2019].

Laskin DL, Pendino KJ (1995) Macrophages and Inflammatory Mediators in Tissue Injury. Annu Rev Pharmacol Toxicol 35:655-677 Available at: http://www.ncbi.nlm.nih.gov/pubmed/7598511 [Accessed August 12, 2017].

Lee CYD, Landreth GE (2010) The role of microglia in amyloid clearance from the AD brain. J Neural Transm 117:949-960 Available at: http://www.ncbi.nlm.nih.gov/pubmed/20552234 [Accessed August 9, 2018].

Lee Y, Andaloussi S El, Wood MJA (2012a) Exosomes and microvesicles: extracellular vesicles for genetic information transfer and gene therapy. Hum Mol Genet 21:R125-R134 Available at: https://oup.silverchaircdn.com/oup/backfile/Content_public/Journal/hmg/21/R1/10.1093/hmg/dds317/2/dds317.p 
df?Expires $=1501692232 \&$ Signature $=$ CufUkuolAsCDJuyvphs7BZ0FxPUq9VkTxeWcc59X jAneU 1Yk8pPjJoM04o5v0LPzkI aiNaPBpEq2c-4UXe-

BGIZ9Oj6hEpr ijjHOBHdONL83LUtgh [Accessed August 1, 2017].

Lee Y, Morrison BM, Li Y, Lengacher S, Farah MH, Hoffman PN, Liu Y, Tsingalia A, Jin L, Zhang P-W, Pellerin L, Magistretti PJ, Rothstein JD (2012b) Oligodendroglia metabolically support axons and contribute to neurodegeneration. Nature 487:443-448 Available at: http://www.ncbi.nlm.nih.gov/pubmed/22801498 [Accessed August 24, 2018].

Leng J, Goldstein DR (2010) Impact of aging on viral infections. Microbes Infect 12:1120-1124 Available at: http://www.ncbi.nlm.nih.gov/pubmed/20849976 [Accessed January 20, 2019].

Lennicke C, Rahn J, Lichtenfels R, Wessjohann LA, Seliger B (2015) Hydrogen peroxide production, fate and role in redox signaling of tumor cells. Cell Commun Signal 13:39 Available at: http://www.ncbi.nlm.nih.gov/pubmed/26369938 [Accessed November 28, 2018].

Lewis BP, Burge CB, Bartel DP (2005) Conserved Seed Pairing, Often Flanked by Adenosines, Indicates that Thousands of Human Genes are MicroRNA Targets. Cell 120:15-20 Available at: http://www.ncbi.nlm.nih.gov/pubmed/15652477 [Accessed January 2, 2019].

Li JJ, Wang B, Kodali MC, Chen C, Kim E, Patters BJ, Lan L, Kumar S, Wang X, Yue J, Liao F-F (2018) In vivo evidence for the contribution of peripheral circulating inflammatory exosomes to neuroinflammation. J Neuroinflammation 15:8 Available at: https://jneuroinflammation.biomedcentral.com/articles/10.1186/s12974-017-1038-8 [Accessed December 16, 2018].

Li Q, Zhang T, Wang J, Zhang Z, Zhai Y, Yang G-Y, Sun X (2014) Rapamycin attenuates mitochondrial dysfunction via activation of mitophagy in experimental ischemic stroke. Biochem Biophys Res Commun 444:182-188 Available at: https://www.sciencedirect.com/science/article/pii/S0006291X14000473 [Accessed January 6, 2019].

Li X, Wang Z, Tan L, Wang Y, Lu C, Chen R, Zhang S, Gao Y, Liu Y, Yin Y, Liu X, Liu E, Yang Y, Hu Y, Xu Z, Xu F, Wang J, Liu G-P, Wang J-Z (2017) Correcting miR92a-vGATMediated GABAergic Dysfunctions Rescues Human Tau-Induced Anxiety in Mice. Mol Ther 25:140-152 Available at: http://www.ncbi.nlm.nih.gov/pubmed/28129110 [Accessed August 12, 2017].

Liang J, Yu Y, Wang B, Lu B, Zhang J, Zhang H, Ge P, Liang J, Yu Y, Wang B, Lu B, Zhang J, Zhang H, Ge P (2013) Ginsenoside Rb1 Attenuates Oxygen-Glucose Deprivation-Induced Apoptosis in SH-SY5Y Cells via Protection of Mitochondria and Inhibition of AIF and Cytochrome c Release. Molecules 18:12777-12792 Available at: http://www.mdpi.com/1420-3049/18/10/12777 [Accessed January 6, 2019].

Liang WS, Reiman EM, Valla J, Dunckley T, Beach TG, Grover A, Niedzielko TL, Schneider LE, Mastroeni D, Caselli R, Kukull W, Morris JC, Hulette CM, Schmechel D, Rogers J, Stephan DA (2008) Alzheimer's disease is associated with reduced expression of energy metabolism genes in posterior cingulate neurons. Proc Natl Acad Sci 105:4441-4446 Available at: http://www.ncbi.nlm.nih.gov/pubmed/18332434 [Accessed August 21, 2018].

Liao Y-F, Wang B-J, Cheng H-T, Kuo L-H, Wolfe MS (2004) Tumor Necrosis Factor- $\alpha$, Interleukin-1 $\beta$, and Interferon- $\gamma$ Stimulate $\gamma$-Secretase-mediated Cleavage of Amyloid Precursor Protein through a JNK-dependent MAPK Pathway. J Biol Chem 279:49523- 
49532 Available at: http://www.ncbi.nlm.nih.gov/pubmed/15347683 [Accessed November $1,2018]$.

Liu DZ, Jickling GC, Ander BP, Hull H, Zhan X, Cox C, Shroff N, Dykstra-Aiello C, Stamova B, Sharp FR (2016a) Elevating microRNA-122 in blood improves outcomes after temporary middle cerebral artery occlusion in rats. J Cereb Blood Flow Metab 36:1374-1383 Available at: http://www.ncbi.nlm.nih.gov/pubmed/26661204 [Accessed August 13, 2017].

Liu W, Cai H, Lin M, Zhu L, Gao L, Zhong R, Bi S, Xue Y, Shang X (2016b) MicroRNA-107 prevents amyloid-beta induced blood-brain barrier disruption and endothelial cell dysfunction by targeting Endophilin-1. Exp Cell Res 343:248-257 Available at: http://www.ncbi.nlm.nih.gov/pubmed/27038654 [Accessed October 30, 2017].

Liu Y-P, Lin H-I, Tzeng S-F (2005) Tumor necrosis factor- $\alpha$ and interleukin-18 modulate neuronal cell fate in embryonic neural progenitor culture. Brain Res 1054:152-158 Available at: http://www.ncbi.nlm.nih.gov/pubmed/16054598 [Accessed January 2, 2019].

Liu Y, Levine B (2015) Autosis and autophagic cell death: the dark side of autophagy. Cell Death Differ 22:367-376 Available at: http://www.nature.com/articles/cdd2014143 [Accessed January 7, 2019].

Livak KJ, Schmittgen TD (2001) Analysis of Relative Gene Expression Data Using Real-Time Quantitative PCR and the 2- $\Delta \Delta \mathrm{CT}$ Method. Methods 25:402-408 Available at: http://www.ncbi.nlm.nih.gov/pubmed/11846609 [Accessed August 23, 2018].

López-Collazo E, del Fresno C (2013) Pathophysiology of endotoxin tolerance: mechanisms and clinical consequences. Crit Care 17:242 Available at: http://www.ncbi.nlm.nih.gov/pubmed/24229432 [Accessed January 8, 2019].

Lopez-Ramirez MA, Wu D, Pryce G, Simpson JE, Reijerkerk A, King-Robson J, Kay O, de Vries HE, Hirst MC, Sharrack B, Baker D, Male DK, Michael GJ, Romero IA (2014) MicroRNA-155 negatively affects blood-brain barrier function during neuroinflammation. FASEB J 28:2551-2565 Available at: http://www.ncbi.nlm.nih.gov/pubmed/24604078 [Accessed October 30, 2017].

Lötvall J, Hill AF, Hochberg F, Buzás EI, Di Vizio D, Gardiner C, Gho YS, Kurochkin I V., Mathivanan S, Quesenberry P, Sahoo S, Tahara H, Wauben MH, Witwer KW, Théry C (2014) Minimal experimental requirements for definition of extracellular vesicles and their functions: a position statement from the International Society for Extracellular Vesicles. J Extracell Vesicles 3:26913 Available at: https://www.tandfonline.com/doi/full/10.3402/jev.v3.26913 [Accessed July 22, 2018].

Lotvall J, Valadi H (2007) Cell to cell signalling via exosomes through esRNA. Cell Adh Migr 1:156-158 Available at: http://www.ncbi.nlm.nih.gov/pubmed/19262134 [Accessed January 2, 2019].

Lu L-F, Boldin MP, Chaudhry A, Lin L-L, Taganov KD, Hanada T, Yoshimura A, Baltimore D, Rudensky AY (2010) Function of miR-146a in Controlling Treg Cell-Mediated Regulation of Th1 Responses. Cell 142:914-929 Available at: http://www.ncbi.nlm.nih.gov/pubmed/20850013 [Accessed August 23, 2018].

Luarte A, Cisternas P, Caviedes A, Batiz LF, Lafourcade C, Wyneken U, Henzi R (2017) Astrocytes at the Hub of the Stress Response: Potential Modulation of Neurogenesis by miRNAs in Astrocyte-Derived Exosomes. Stem Cells Int 2017:1-13 Available at: https://www.hindawi.com/journals/sci/2017/1719050/ [Accessed January 5, 2019]. 
Luketic L, Delanghe J, Sobol PT, Yang P, Frotten E, Mossman KL, Gauldie J, Bramson J, Wan Y (2007) Antigen presentation by exosomes released from peptide-pulsed dendritic cells is not suppressed by the presence of active CTL. J Immunol 179:5024-5032 Available at: http://www.ncbi.nlm.nih.gov/pubmed/17911587 [Accessed August 2, 2017].

Lukiw WJ (2007) Micro-RNA speciation in fetal, adult and Alzheimer's disease hippocampus. Neuroreport 18:297-300 Available at: http://www.ncbi.nlm.nih.gov/pubmed/17314675 [Accessed August 23, 2018].

Lukiw WJ, Alexandrov PN (2012) Regulation of Complement Factor H (CFH) by Multiple miRNAs in Alzheimer's Disease (AD) Brain. Mol Neurobiol 46:11-19 Available at: http://www.ncbi.nlm.nih.gov/pubmed/22302353 [Accessed August 9, 2018].

Lukiw WJ, Surjyadipta B, Dua P, Alexandrov PN (2012) Common micro RNAs (miRNAs) target complement factor H (CFH) regulation in Alzheimer's disease (AD) and in agerelated macular degeneration (AMD). Int J Biochem Mol Biol 3:105-116 Available at: http://www.ncbi.nlm.nih.gov/pubmed/22509485 [Accessed August 23, 2018].

Lukiw WJ, Zhao Y, Cui JG (2008) An NF-кB-sensitive Micro RNA-146a-mediated Inflammatory Circuit in Alzheimer Disease and in Stressed Human Brain Cells. J Biol Chem 283:31315-31322 Available at: http://www.ncbi.nlm.nih.gov/pubmed/18801740 [Accessed August 21, 2018].

Lv L-L, Wu W-J, Feng Y, Li Z-L, Tang T-T, Liu B-C (2018) Therapeutic application of extracellular vesicles in kidney disease: promises and challenges. J Cell Mol Med 22:728737 Available at: http://www.ncbi.nlm.nih.gov/pubmed/29083099 [Accessed January 6, 2019].

Ma Q, Zhao H, Tao Z, Wang R, Liu P, Han Z, Ma S, Luo Y, Jia J (2016) MicroRNA-181c Exacerbates Brain Injury in Acute Ischemic Stroke. Aging Dis 7:705-714 Available at: http://www.ncbi.nlm.nih.gov/pubmed/28053821 [Accessed January 3, 2019].

MacEwan DJ (2002) TNF receptor subtype signalling: differences and cellular consequences. Cell Signal 14:477-492 Available at: http://www.ncbi.nlm.nih.gov/pubmed/11897488 [Accessed January 2, 2019].

Mack M, Kleinschmidt A, Brühl H, Klier C, Nelson PJ, Cihak J, Plachý J, Stangassinger M, Erfle V, Schlöndorff D (2000) Transfer of the chemokine receptor CCR5 between cells by membrane-derivedmicroparticles: A mechanism for cellular human immunodeficiency virus 1 infection. Nat Med 6:769-775 Available at: http://www.nature.com/doifinder/10.1038/77498 [Accessed April 28, 2018].

Mackenzie K, Foot NJ, Anand S, Dalton HE, Chaudhary N, Collins BM, Mathivanan S, Kumar $S$ (2016) Regulation of the divalent metal ion transporter via membrane budding. Cell Discov 2:16011 Available at: http://www.nature.com/articles/celldisc201611 [Accessed October 27, 2018].

Maiuri AR, Li H, Stein BD, Tennessen JM, O’Hagan HM (2018) Inflammation-induced DNA methylation of DNA polymerase gamma alters the metabolic profile of colon tumors. Cancer Metab 6:9 Available at: http://www.ncbi.nlm.nih.gov/pubmed/30002826 [Accessed January 3, 2019].

Marquardt L, Ruf A, Mansmann U, Winter R, Schuler M, Buggle F, Mayer H, Grau AJ (2002) Course of platelet activation markers after ischemic stroke. Stroke 33:2570-2574 Available at: http://www.ncbi.nlm.nih.gov/pubmed/12411644 [Accessed January 6, 2019]. 
Martin-Villalba A, Herr I, Jeremias I, Hahne M, Brandt R, Vogel J, Schenkel J, Herdegen T, Debatin KM (1999) CD95 ligand (Fas-L/APO-1L) and tumor necrosis factor-related apoptosis-inducing ligand mediate ischemia-induced apoptosis in neurons. J Neurosci 19:3809-3817 Available at: http://www.ncbi.nlm.nih.gov/pubmed/10234013 [Accessed January 7, 2019].

Marzesco A-M, Janich P, Wilsch-Bräuninger M, Dubreuil V, Langenfeld K, Corbeil D, Huttner WB (2005) Release of extracellular membrane particles carrying the stem cell marker prominin-1 (CD133) from neural progenitors and other epithelial cells. J Cell Sci 118:28492858 Available at: http://www.ncbi.nlm.nih.gov/pubmed/15976444 [Accessed January 5, 2019].

Matsumoto J, Stewart T, Banks WA, Zhang J (2018) The Transport Mechanism of Extracellular Vesicles at the Blood-Brain Barrier. Curr Pharm Des 23:6206-6214 Available at: http://www.ncbi.nlm.nih.gov/pubmed/28914201 [Accessed January 6, 2019].

Maurer LM, Tomasini-Johansson BR, Mosher DF (2010) Emerging roles of fibronectin in thrombosis. Thromb Res 125:287-291 Available at: http://www.ncbi.nlm.nih.gov/pubmed/20116835 [Accessed January 3, 2019].

McEwen B (2014) The Influence of Diet and Nutrients on Platelet Function. Semin Thromb Hemost 40:214-226 Available at: http://www.ncbi.nlm.nih.gov/pubmed/24497119 [Accessed January 6, 2019].

McKenzie AJ, Hoshino D, Hong NH, Cha DJ, Franklin JL, Coffey RJ, Patton JG, Weaver AM (2016) KRAS-MEK Signaling Controls Ago2 Sorting into Exosomes. Cell Rep 15:978-987 Available at: http://www.ncbi.nlm.nih.gov/pubmed/27117408 [Accessed October 28, 2018].

Meisel C, Schwab JM, Prass K, Meisel A, Dirnagl U (2005) Central nervous system injuryinduced immune deficiency syndrome. Nat Rev Neurosci 6:775-786 Available at: http://www.nature.com/articles/nrn1765 [Accessed January 3, 2019].

Meyer A, Laverny G, Bernardi L, Charles AL, Alsaleh G, Pottecher J, Sibilia J, Geny B (2018) Mitochondria: An Organelle of Bacterial Origin Controlling Inflammation. Front Immunol 9:536 Available at: http://journal.frontiersin.org/article/10.3389/fimmu.2018.00536/full [Accessed January 2, 2019].

Micheau O, Tschopp J (2003) Induction of TNF Receptor I-Mediated Apoptosis via Two Sequential Signaling Complexes. Cell 114:181-190 Available at: https://www.sciencedirect.com/science/article/pii/S009286740300521X [Accessed January 7, 2019].

Migita K, Iwanaga N, Izumi Y, Kawahara C, Kumagai K, Nakamura T, Koga T, Kawakami A (2017) TNF- $\alpha$-induced miR-155 regulates IL-6 signaling in rheumatoid synovial fibroblasts. BMC Res Notes 10:403 Available at: http://www.ncbi.nlm.nih.gov/pubmed/28807007 [Accessed January 3, 2019].

Monje ML, Toda H, Palmer TD (2003) Inflammatory Blockade Restores Adult Hippocampal Neurogenesis. Science (80- ) 302:1760-1765 Available at: http://www.ncbi.nlm.nih.gov/pubmed/14615545 [Accessed January 2, 2019].

Montagne A, Barnes SR, Sweeney MD, Halliday MR, Sagare AP, Zhao Z, Toga AW, Jacobs RE, Liu CY, Amezcua L, Harrington MG, Chui HC, Law M, Zlokovic B V (2015) BloodBrain Barrier Breakdown in the Aging Human Hippocampus HHS Public Access. Neuron January 2112 Available at: 
https://www.ncbi.nlm.nih.gov/pmc/articles/PMC4350773/pdf/nihms651289.pdf [Accessed July 8, 2017].

Montecalvo A, Larregina AT, Shufesky WJ, Beer Stolz D, Sullivan MLG, Karlsson JM, Baty CJ, Gibson GA, Erdos G, Wang Z, Milosevic J, Tkacheva OA, Divito SJ, Jordan R, LyonsWeiler J, Watkins SC, Morelli AE (2012) Mechanism of transfer of functional microRNAs between mouse dendritic cells via exosomes. Blood 119:756-766 Available at: http://www.ncbi.nlm.nih.gov/pubmed/22031862 [Accessed August 2, 2017].

Montgomery SL, Bowers WJ (2012) Tumor Necrosis Factor-alpha and the Roles it Plays in Homeostatic and Degenerative Processes Within the Central Nervous System. J Neuroimmune Pharmacol 7:42-59 Available at: http://www.ncbi.nlm.nih.gov/pubmed/21728035 [Accessed January 2, 2019].

Morris GP, Clark IA, Zinn R, Vissel B (2013) Microglia: A new frontier for synaptic plasticity, learning and memory, and neurodegenerative disease research. Neurobiol Learn Mem 105:40-53 Available at: http://www.ncbi.nlm.nih.gov/pubmed/23850597 [Accessed August 4, 2017].

Mosconi L, Mistur R, Switalski R, Tsui WH, Glodzik L, Li Y, Pirraglia E, De Santi S, Reisberg B, Wisniewski T, de Leon MJ (2009) FDG-PET changes in brain glucose metabolism from normal cognition to pathologically verified Alzheimer's disease. Eur J Nucl Med Mol Imaging 36:811-822 Available at: http://www.ncbi.nlm.nih.gov/pubmed/19142633 [Accessed January 1, 2019].

Mosconi L, Pupi A, De Leon MJ (2008) Brain Glucose Hypometabolism and Oxidative Stress in Preclinical Alzheimer's Disease. Ann N Y Acad Sci 1147:180-195 Available at: http://www.ncbi.nlm.nih.gov/pubmed/19076441 [Accessed August 9, 2018].

Mosconi L, Sorbi S, de Leon MJ, Li Y, Nacmias B, Myoung PS, Tsui W, Ginestroni A, Bessi V, Fayyazz M, Caffarra P, Pupi A (2006) Hypometabolism exceeds atrophy in presymptomatic early-onset familial Alzheimer's disease. J Nucl Med 47:1778-1786 Available at: http://www.ncbi.nlm.nih.gov/pubmed/17079810 [Accessed August 9, 2018].

Mosher KI, Wyss-Coray T (2014) Microglial dysfunction in brain aging and Alzheimer's disease. Biochem Pharmacol 88:594-604 Available at: http://www.ncbi.nlm.nih.gov/pubmed/24445162 [Accessed January 1, 2019].

Mustapic M, Eitan E, Werner JK, Berkowitz ST, Lazaropoulos MP, Tran J, Goetzl EJ, Kapogiannis D, Kapogiannis D (2017) Plasma Extracellular Vesicles Enriched for Neuronal Origin: A Potential Window into Brain Pathologic Processes. Front Neurosci 11:278 Available at: http://www.ncbi.nlm.nih.gov/pubmed/28588440 [Accessed January 5, 2019].

Nabhan JF, Hu R, Oh RS, Cohen SN, Lu Q (2012) Formation and release of arrestin domaincontaining protein 1-mediated microvesicles (ARMMs) at plasma membrane by recruitment of TSG101 protein. PNAS 109:4146-4151 Available at: http://www.pnas.org/content/pnas/109/11/4146.full.pdf [Accessed April 23, 2018].

Neubert J, Glumm J (2016) Promoting neuronal regeneration using extracellular vesicles loaded with superparamagnetic iron oxide nanoparticles. Neural Regen Res 11:61-63 Available at: http://www.ncbi.nlm.nih.gov/pubmed/26981080 [Accessed January 6, 2019].

Neumann H, Schweigreiter R, Yamashita T, Rosenkranz K, Wekerle H, Barde Y-A (2002) Tumor necrosis factor inhibits neurite outgrowth and branching of hippocampal neurons by a rho-dependent mechanism. J Neurosci 22:854-862 Available at: 
http://www.ncbi.nlm.nih.gov/pubmed/11826115 [Accessed January 2, 2019].

Nguyen LS, Fregeac J, Bole-Feysot C, Cagnard N, Iyer A, Anink J, Aronica E, Alibeu O, Nitschke P, Colleaux L (2018) Role of miR-146a in neural stem cell differentiation and neural lineage determination: relevance for neurodevelopmental disorders. Mol Autism 9:38 Available at: http://www.ncbi.nlm.nih.gov/pubmed/29951184 [Accessed November 25, 2018].

Nicolas M, Hassan BA (2014) Amyloid precursor protein and neural development. Development 141:2543-2548 Available at: http://www.ncbi.nlm.nih.gov/pubmed/24961795 [Accessed August 12, 2017].

Nimmerjahn A, Kirchhoff F, Helmchen F (2005) Resting Microglial Cells Are Highly Dynamic Surveillants of Brain Parenchyma in Vivo. Science (80- ) 308:1314-1318 Available at: http://www.ncbi.nlm.nih.gov/pubmed/15831717 [Accessed August 4, 2017].

Nottet HS, Persidsky Y, Sasseville VG, Nukuna AN, Bock P, Zhai QH, Sharer LR, McComb RD, Swindells S, Soderland C, Gendelman HE (1996) Mechanisms for the transendothelial migration of HIV-1-infected monocytes into brain. J Immunol 156:1284-1295 Available at: http://www.ncbi.nlm.nih.gov/pubmed/8558009 [Accessed August 3, 2017].

Obregon C, Rothen-Rutishauser B, Gitahi SK, Gehr P, Nicod LP (2006) Exovesicles from Human Activated Dendritic Cells Fuse with Resting Dendritic Cells, Allowing Them to Present Alloantigens. Am J Pathol 169:2127-2136 Available at: https://www.sciencedirect.com/science/article/pii/S0002944010626727?via\%3Dihub\#fig1 [Accessed October 29, 2018].

Oeckinghaus A, Ghosh S (2009) The NF-kappaB family of transcription factors and its regulation. Cold Spring Harb Perspect Biol 1:a000034 Available at: http://www.ncbi.nlm.nih.gov/pubmed/20066092 [Accessed May 23, 2018].

Okano M, Bell DW, Haber DA, Li E (1999) DNA methyltransferases Dnmt3a and Dnmt3b are essential for de novo methylation and mammalian development. Cell 99:247-257 Available at: http://www.ncbi.nlm.nih.gov/pubmed/10555141 [Accessed January 3, 2019].

Olivieri F, Rippo MR, Procopio AD, Fazioli F (2013) Circulating inflamma-miRs in aging and age-related diseases. Front Genet 4:121 Available at: http://www.ncbi.nlm.nih.gov/pubmed/23805154 [Accessed January 2, 2019].

Orrenius S, Zhivotovsky B (2005) Cardiolipin oxidation sets cytochrome c free. Nat Chem Biol 1:188-189 Available at: http://www.nature.com/articles/nchembio0905-188 [Accessed January 5, 2019].

Ostrowski M et al. (2010) Rab27a and Rab27b control different steps of the exosome secretion pathway. Nat Cell Biol 12:19-30 Available at: http://www.nature.com/doifinder/10.1038/ncb2000 [Accessed April 23, 2018].

Ott M, Robertson JD, Gogvadze V, Zhivotovsky B, Orrenius S (2002) Cytochrome c release from mitochondria proceeds by a two-step process. Proc Natl Acad Sci U S A 99:12591263 Available at: http://www.ncbi.nlm.nih.gov/pubmed/11818574 [Accessed January 7, 2019].

Ouyang Y-B, Lu Y, Yue S, Xu L-J, Xiong X-X, White RE, Sun X, Giffard RG, Stamler J, Ferguson D, Roberts C, Hwang J, Trendler T, Kennan R, Suzuki Y, Reardon C, Hickey W, Chen L, McLeod R (2012) miR-181 regulates GRP78 and influences outcome from cerebral ischemia in vitro and in vivo. Neurobiol Dis 45:555-563 Available at: 
http://linkinghub.elsevier.com/retrieve/pii/S0969996111003226 [Accessed August 3, 2017].

Paganini-Hill A, Lozano E, Fischberg G, Perez Barreto M, Rajamani K, Ameriso SF, Heseltine

PNR, Fisher M (2003) Infection and Risk of Ischemic Stroke. Stroke 34:452-457 Available at: https://www.ahajournals.org/doi/10.1161/01.STR.0000053451.28410.98 [Accessed December 16, 2018].

Pan BT, Johnstone RM (1983) Fate of the transferrin receptor during maturation of sheep reticulocytes in vitro: selective externalization of the receptor. Cell 33:967-978 Available at: http://www.ncbi.nlm.nih.gov/pubmed/6307529 [Accessed January 5, 2019].

Pan BT, Teng K, Wu C, Adam M, Johnstone RM (1985) Electron microscopic evidence for externalization of the transferrin receptor in vesicular form in sheep reticulocytes. J Cell Biol 101:942-948 Available at: http://www.ncbi.nlm.nih.gov/pubmed/2993317 [Accessed May 22, 2018].

Pan W, Kastin AJ (2007) Tumor necrosis factor and stroke: role of the blood-brain barrier. Prog Neurobiol 83:363-374 Available at: http://www.ncbi.nlm.nih.gov/pubmed/17913328 [Accessed January 3, 2019].

Papadakis M, Hadley G, Xilouri M, Hoyte LC, Nagel S, McMenamin MM, Tsaknakis G, Watt SM, Drakesmith CW, Chen R, Wood MJA, Zhao Z, Kessler B, Vekrellis K, Buchan AM (2013) Tsc1 (hamartin) confers neuroprotection against ischemia by inducing autophagy. Nat Med 19:351-357 Available at: http://www.nature.com/articles/nm.3097 [Accessed January 3,2019$]$.

Paresce DM, Chung H, Maxfield FR (1997) Slow degradation of aggregates of the Alzheimer's disease amyloid beta-protein by microglial cells. J Biol Chem 272:29390-29397 Available at: http://www.ncbi.nlm.nih.gov/pubmed/9361021 [Accessed August 9, 2018].

Pawluczyk IZ, Harris KP (1998) Cytokine interactions promote synergistic fibronectin accumulation by mesangial cells. Kidney Int 54:62-70 Available at: http://www.ncbi.nlm.nih.gov/pubmed/9648064 [Accessed January 3, 2019].

Pena-Philippides JC, Caballero-Garrido E, Lordkipanidze T, Roitbak T (2016) In vivo inhibition of miR-155 significantly alters post-stroke inflammatory response. J Neuroinflammation 13:287 Available at: http://www.ncbi.nlm.nih.gov/pubmed/27829437 [Accessed January 3, 2019].

Perry RT, Collins JS, Wiener H, Acton R, Go RC (2001) The role of TNF and its receptors in Alzheimer's disease. Neurobiol Aging 22:873-883 Available at: http://www.ncbi.nlm.nih.gov/pubmed/11754994 [Accessed August 4, 2017].

Persidsky Y, Gendelman HE (1997) Development of laboratory and animal model systems for HIV-1 encephalitis and its associated dementia. J Leukoc Biol 62:100-106 Available at: http://www.ncbi.nlm.nih.gov/pubmed/9226000 [Accessed August 3, 2017].

Phinney DG, Di Giuseppe M, Njah J, Sala E, Shiva S, St Croix CM, Stolz DB, Watkins SC, Di YP, Leikauf GD, Kolls J, Riches DWH, Deiuliis G, Kaminski N, Boregowda S V., McKenna DH, Ortiz LA (2015) Mesenchymal stem cells use extracellular vesicles to outsource mitophagy and shuttle microRNAs. Nat Commun 6:8472 Available at: http://www.nature.com/articles/ncomms9472 [Accessed November 16, 2018].

Plaza-Zabala A, Sierra-Torre V, Sierra A (2017) Autophagy and Microglia: Novel Partners in Neurodegeneration and Aging. Int J Mol Sci 18 Available at: http://www.ncbi.nlm.nih.gov/pubmed/28282924 [Accessed January 7, 2019]. 
Pogue AI, Lukiw WJ (2018) Up-regulated Pro-inflammatory MicroRNAs (miRNAs) in Alzheimer's disease (AD) and Age-Related Macular Degeneration (AMD). Cell Mol Neurobiol 38:1021-1031 Available at: http://www.ncbi.nlm.nih.gov/pubmed/29302837 [Accessed November 25, 2018].

Polanco JC, Li C, Durisic N, Sullivan R, Götz J (2018) Exosomes taken up by neurons hijack the endosomal pathway to spread to interconnected neurons. Acta Neuropathol Commun 6:10 Available at: http://www.ncbi.nlm.nih.gov/pubmed/29448966 [Accessed January 5, 2019].

Pongratz G, Straub RH (2014) The sympathetic nervous response in inflammation. Arthritis Res Ther 16:504 Available at: http://www.ncbi.nlm.nih.gov/pubmed/25789375 [Accessed January 3, 2019].

Ponomarev ED, Veremeyko T, Barteneva N, Krichevsky AM, Weiner HL (2011) MicroRNA124 promotes microglia quiescence and suppresses EAE by deactivating macrophages via the C/EBP- $\alpha-P U .1$ pathway. Nat Med 17:64-70 Available at: https://www.nature.com/nm/journal/v17/n1/pdf/nm.2266.pdf [Accessed August 3, 2017].

Potolicchio I, Carven GJ, Xu X, Stipp C, Riese RJ, Stern LJ, Santambrogio L (2005) Proteomic analysis of microglia-derived exosomes: metabolic role of the aminopeptidase CD13 in neuropeptide catabolism. J Immunol 175:2237-2243 Available at: http://www.ncbi.nlm.nih.gov/pubmed/16081791 [Accessed January 5, 2019].

Prat A, Biernacki K, Wosik K, Antel JP (2001) Glial cell influence on the human blood-brain barrier. Glia 36:145-155.

Pristerà A, Saraulli D, Farioli-Vecchioli S, Strimpakos G, Costanzi M, di Certo MG, Cannas S, Ciotti MT, Tirone F, Mattei E, Cestari V, Canu N (2013) Impact of N-tau on adult hippocampal neurogenesis, anxiety, and memory. Neurobiol Aging 34:2551-2563 Available at: http://www.ncbi.nlm.nih.gov/pubmed/23769395 [Accessed August 12, 2017].

Putz U, Howitt J, Lackovic J, Foot N, Kumar S, Silke J, Tan S-S (2008) Nedd4 familyinteracting protein 1 (Ndfip1) is required for the exosomal secretion of Nedd4 family proteins. J Biol Chem 283:32621-32627 Available at: http://www.ncbi.nlm.nih.gov/pubmed/18819914 [Accessed January 5, 2019].

Quinn SR, O'Neill LA (2011) A trio of microRNAs that control Toll-like receptor signalling. Int Immunol 23:421-425 Available at: http://www.ncbi.nlm.nih.gov/pubmed/21652514 [Accessed January 2, 2019].

Qureshi IA, Mehler MF (2010) Emerging role of epigenetics in stroke: part 1: DNA methylation and chromatin modifications. Arch Neurol 67:1316-1322 Available at: http://www.ncbi.nlm.nih.gov/pubmed/21060009 [Accessed January 3, 2019].

Rajendran L, Honsho M, Zahn TR, Keller P, Geiger KD, Verkade P, Simons K (2006) Alzheimer's disease beta-amyloid peptides are released in association with exosomes. Proc Natl Acad Sci 103:11172-11177 Available at:

http://www.ncbi.nlm.nih.gov/pubmed/16837572 [Accessed January 5, 2019].

Ramachandran S, Palanisamy V (2012) Horizontal transfer of RNAs: exosomes as mediators of intercellular communication. Wiley Interdiscip Rev RNA 3:286-293 Available at: http://www.ncbi.nlm.nih.gov/pubmed/22012863 [Accessed January 2, 2019].

Raposo G, Stoorvogel W (2013) Extracellular vesicles: Exosomes, microvesicles, and friends. J Cell Biol 200:373-383 Available at: http://www.ncbi.nlm.nih.gov/pubmed/23420871 [Accessed August 2, 2017]. 
Raschzok N, Werner W, Sallmon H, Billecke N, Dame C, Neuhaus P, Sauer IM (2011)

Temporal expression profiles indicate a primary function for microRNA during the peak of DNA replication after rat partial hepatectomy. Am J Physiol Regul Integr Comp Physiol 300:1363-1372 Available at: www.microrna.org/ [Accessed August 24, 2018].

Ratajczak J, Wysoczynski M, Hayek F, Janowska-Wieczorek A, Ratajczak MZ (2006)

Membrane-derived microvesicles: important and underappreciated mediators of cell-to-cell communication. Leukemia 20:1487-1495 Available at: http://www.nature.com/articles/2404296 [Accessed August 24, 2018].

Rehwinkel J, Behm-Ansmant I, Gatfield D, Izaurralde E (2005) A crucial role for GW182 and the DCP1:DCP2 decapping complex in miRNA-mediated gene silencing. RNA 11:1640 1647 Available at: http://www.ncbi.nlm.nih.gov/pubmed/16177138 [Accessed January 2, 2019].

Rhein V, Baysang G, Rao S, Meier F, Bonert A, Müller-Spahn F, Eckert A (2009) Amyloid-beta Leads to Impaired Cellular Respiration, Energy Production and Mitochondrial Electron Chain Complex Activities in Human Neuroblastoma Cells. Cell Mol Neurobiol 29:10631071 Available at: http://www.ncbi.nlm.nih.gov/pubmed/19350381 [Accessed August 10, 2018].

Ricart KC, Fiszman ML (2001) Hydrogen Peroxide-Induced Neurotoxicity in Cultured Cortical Cells Grown in Serum-Free and Serum-Containing Media. Available at: https://link.springer.com/content/pdf/10.1023\%2FA\%3A1011660001941.pdf [Accessed November 28, 2018].

Rippo MR, Olivieri F, Monsurrò V, Prattichizzo F, Albertini MC, Procopio AD (2014) MitomiRs in human inflamm-aging: A hypothesis involving miR-181a, miR-34a and miR146a. Exp Gerontol 56:154-163 Available at: http://www.ncbi.nlm.nih.gov/pubmed/24607549 [Accessed August 9, 2018].

Riske L, Thomas RK, Baker GB, Dursun SM (2017) Lactate in the brain: an update on its relevance to brain energy, neurons, glia and panic disorder. Ther Adv Psychopharmacol 7:85-89 Available at: http://www.ncbi.nlm.nih.gov/pubmed/28255438 [Accessed January $5,2019]$.

Rock RB, Gekker G, Hu S, Sheng WS, Cheeran M, Lokensgard JR, Peterson PK (2004) Role of Microglia in Central Nervous System Infections. Clin Microbiol Rev 17:942-964 Available at: http://www.ncbi.nlm.nih.gov/pubmed/15489356 [Accessed August 4, 2017].

Rogers J, Cooper NR, Webster S, Schultz J, McGeer PL, Styren SD, Civin WH, Brachova L, Bradt B, Ward P (1992) Complement activation by beta-amyloid in Alzheimer disease. Proc Natl Acad Sci U S A 89:10016-10020 Available at: http://www.ncbi.nlm.nih.gov/pubmed/1438191 [Accessed August 12, 2017].

Rosenzweig HL, Lessov NS, Henshall DC, Minami M, Simon RP, Stenzel-Poore MP (2004) Endotoxin Preconditioning Prevents Cellular Inflammatory Response During Ischemic Neuroprotection in Mice. Stroke 35:2576-2581 Available at: http://www.ncbi.nlm.nih.gov/pubmed/15375302 [Accessed January 8, 2019].

Rosenzweig HL, Minami M, Lessov NS, Coste SC, Stevens SL, Henshall DC, Meller R, Simon RP, Stenzel-Poore MP (2007) Endotoxin Preconditioning Protects against the Cytotoxic Effects of TNF $\alpha$ after Stroke: A Novel Role for TNF $\alpha$ in LPS-Ischemic Tolerance. J Cereb Blood Flow Metab 27:1663-1674 Available at: 
http://journals.sagepub.com/doi/10.1038/sj.jcbfm.9600464 [Accessed January 8, 2019].

Rozmyslowicz T, Majka M, Kijowski J, Murphy SL, Conover DO, Poncz M, Ratajczak J, Gaulton GN, Ratajczak MZ (2003) Platelet- and megakaryocyte-derived microparticles transfer CXCR4 receptor to CXCR4-null cells and make them susceptible to infection by X4-HIV. AIDS 17:33-42 Available at: http://www.ncbi.nlm.nih.gov/pubmed/12478067 [Accessed May 29, 2018].

Rufino-Ramos D, Albuquerque PR, Carmona V, Perfeito R, Nobre RJ, Pereira de Almeida L (2017) Extracellular vesicles: Novel promising delivery systems for therapy of brain diseases. J Control Release 262:247-258 Available at: http://www.ncbi.nlm.nih.gov/pubmed/28687495 [Accessed January 6, 2019].

Salvarani C, Morris JM, Giannini C, Brown RD, Christianson T, Hunder GG, Hunder GG (2016) Imaging Findings of Cerebral Amyloid Angiopathy, A $\beta$-Related Angiitis (ABRA), and Cerebral Amyloid Angiopathy-Related Inflammation: A Single-Institution 25-Year Experience. Medicine (Baltimore) 95:e3613 Available at: http://www.ncbi.nlm.nih.gov/pubmed/27196463 [Accessed August 23, 2018].

Saman S, Kim W, Raya M, Visnick Y, Miro S, Saman S, Jackson B, McKee AC, Alvarez VE, Lee NCY, Hall GF (2012) Exosome-associated Tau Is Secreted in Tauopathy Models and Is Selectively Phosphorylated in Cerebrospinal Fluid in Early Alzheimer Disease. J Biol Chem 287:3842-3849 Available at: http://www.ncbi.nlm.nih.gov/pubmed/22057275 [Accessed January 5, 2019].

Sansone P et al. (2017) Packaging and transfer of mitochondrial DNA via exosomes regulate escape from dormancy in hormonal therapy-resistant breast cancer. Proc Natl Acad Sci U S A 114:E9066-E9075 Available at: http://www.ncbi.nlm.nih.gov/pubmed/29073103 [Accessed November 14, 2018].

Santilli F, Vazzana N, Liani R, Guagnano MT, Davì G (2012) Platelet activation in obesity and metabolic syndrome. Obes Rev 13:27-42 Available at: http://www.ncbi.nlm.nih.gov/pubmed/21917110 [Accessed January 6, 2019].

Sardar Sinha M, Ansell-Schultz A, Civitelli L, Hildesjö C, Larsson M, Lannfelt L, Ingelsson M, Hallbeck M (2018) Alzheimer's disease pathology propagation by exosomes containing toxic amyloid-beta oligomers. Acta Neuropathol 136:41-56 Available at: http://link.springer.com/10.1007/s00401-018-1868-1 [Accessed November 21, 2018].

Sarkar S, Jun S, Rellick S, Quintana DD, Cavendish JZ, Simpkins JW (2016) Expression of microRNA-34a in Alzheimer's disease brain targets genes linked to synaptic plasticity, energy metabolism, and resting state network activity. Brain Res 1646:139-151 Available at: https://www.sciencedirect.com/science/article/pii/S0006899316303821 [Accessed August 27, 2018].

Sarkar S, Jun S, Simpkins JW (2015) Estrogen amelioration of A $\beta$-induced defects in mitochondria is mediated by mitochondrial signaling pathway involving ER $\beta$, AKAP and Drp1. Brain Res 1616:101-111.

Sarkar SN, Russell AE, Engler-Chiurazzi EB, Porter KN, Simpkins JW (2018) MicroRNAs and the Genetic Nexus of Brain Aging, Neuroinflammation, Neurodegeneration, and Brain Trauma. Aging Dis:0 Available at:

http://www.aginganddisease.org/EN/10.14336/AD.2018.0409 [Accessed January 3, 2019].

Sas K, Robotka H, Toldi J, Vécsei L (2007) Mitochondria, metabolic disturbances, oxidative 
stress and the kynurenine system, with focus on neurodegenerative disorders. J Neurol Sci 257:221-239 Available at: http://www.ncbi.nlm.nih.gov/pubmed/17462670 [Accessed January 5, 2019].

Saunderson SC, Schuberth PC, Dunn AC, Miller L, Hock BD, MacKay PA, Koch N, Jack RW, McLellan AD (2008) Induction of exosome release in primary B cells stimulated via CD40 and the IL-4 receptor. J Immunol 180:8146-8152 Available at: http://www.ncbi.nlm.nih.gov/pubmed/18523279 [Accessed October 29, 2018].

Schütze S, Wiegmann K, Machleidt T, Krönke M (1995) TNF-induced activation of NF-kappa B. Immunobiology 193:193-203 Available at: http://www.ncbi.nlm.nih.gov/pubmed/8530143 [Accessed November 19, 2018].

Segawa K, Nagata S (2015) An Apoptotic 'Eat Me' Signal: Phosphatidylserine Exposure. Trends Cell Biol 25:639-650 Available at: http://www.ncbi.nlm.nih.gov/pubmed/26437594 [Accessed January 2, 2019].

Selkoe DJ (1994) Normal and Abnormal Biology of the beta-Amyloid Precursor Protein. Annu Rev Neurosci 17:489-517 Available at: http://www.annualreviews.org/doi/10.1146/annurev.ne.17.030194.002421 [Accessed August 12, 2017].

Sessa R, Pietro M Di, Filardo S, Turriziani O (2014) Infectious burden and atherosclerosis: A clinical issue. World J Clin cases 2:240-249 Available at: http://www.ncbi.nlm.nih.gov/pubmed/25032197 [Accessed January 3, 2019].

Shah GN, Mooradian AD (1997) Age-related changes in the blood-brain barrier. Exp Gerontol 32:501-519 Available at: http://linkinghub.elsevier.com/retrieve/pii/S0531556596001581 [Accessed August 4, 2017].

Sharma V, Thakur V, Singh SN, Guleria R (2012) Tumor Necrosis Factor and Alzheimer's Disease: A Cause and Consequence Relationship. Klin Psikofarmakol Bülteni-Bulletin Clin Psychopharmacol 22:86-97 Available at: https://www.tandfonline.com/doi/full/10.5455/bcp.20120112064639 [Accessed January 4, 2019].

Shoji Y, Uedono Y, Ishikura H, Takeyama N, Tanaka T (1995) DNA damage induced by tumour necrosis factor-alpha in L929 cells is mediated by mitochondrial oxygen radical formation. Immunology 84:543-548 Available at: http://www.ncbi.nlm.nih.gov/pubmed/7790027 [Accessed January 7, 2019].

Shors TJ, Miesegaes G, Beylin A, Zhao M, Rydel T, Gould E (2001) Neurogenesis in the adult is involved in the formation of trace memories. Nature 410:372-376 Available at: http://www.ncbi.nlm.nih.gov/pubmed/11268214 [Accessed January 2, 2019].

Simeoli R, Montague K, Jones HR, Castaldi L, Chambers D, Kelleher JH, Vacca V, Pitcher T, Grist J, Al-Ahdal H, Wong L-F, Perretti M, Lai J, Mouritzen P, Heppenstall P, Malcangio M (2017) Exosomal cargo including microRNA regulates sensory neuron to macrophage communication after nerve trauma. Nat Commun 8:1778 Available at: http://www.nature.com/articles/s41467-017-01841-5 [Accessed January 5, 2019].

Simpkins JW, Yi KD, Yang S-H, Dykens JA (2010) Mitochondrial mechanisms of estrogen neuroprotection. Biochim Biophys Acta 1800:1113-1120 Available at: http://www.ncbi.nlm.nih.gov/pubmed/19931595 [Accessed January 5, 2019].

Smith AD (2002) Imaging the progression of Alzheimer pathology through the brain. Proc Natl 
Acad Sci U S A 99:4135-4137 Available at:

http://www.ncbi.nlm.nih.gov/pubmed/11929987 [Accessed August 9, 2018].

Sochocka M, Diniz BS, Leszek J (2017a) Inflammatory Response in the CNS: Friend or Foe? Mol Neurobiol 54:8071-8089 Available at:

http://www.ncbi.nlm.nih.gov/pubmed/27889895 [Accessed August 24, 2018].

Sochocka M, Diniz BS, Leszek J (2017b) Inflammatory Response in the CNS: Friend or Foe? Mol Neurobiol 54:8071-8089.

Song Y, Dou H, Li X, Zhao X, Li Y, Liu D, Ji J, Liu F, Ding L, Ni Y, Hou Y (2017) Exosomal miR-146a Contributes to the Enhanced Therapeutic Efficacy of Interleukin-1 $\beta$-Primed Mesenchymal Stem Cells Against Sepsis. Stem Cells 35:1208-1221 Available at: http://www.ncbi.nlm.nih.gov/pubmed/28090688 [Accessed August 23, 2018].

Soriano-Tárraga C, Jiménez-Conde J, Giralt-Steinhauer E, Mola M, Ois Á, Rodríguez-Campello A, Cuadrado-Godia E, Fernández-Cadenas I, Carrera C, Montaner J, Elosua R, Roquer J, GeneStroke on behalf of, Consortium" "The Spanish Stroke Genetics (2014) Global DNA Methylation of Ischemic Stroke Subtypes Lo AWI, ed. PLoS One 9:e96543 Available at: http://dx.plos.org/10.1371/journal.pone.0096543 [Accessed January 3, 2019].

Soriano-Tárraga C, Mola-Caminal M, Giralt-Steinhauer E, Ois A, Rodríguez-Campello A, Cuadrado-Godia E, Gómez-González A, Vivanco-Hidalgo RM, Fernández-Cadenas I, Cullell N, Roquer J, Jiménez-Conde J (2017) Biological age is better than chronological as predictor of 3-month outcome in ischemic stroke. Neurology 89:830-836 Available at: http://www.ncbi.nlm.nih.gov/pubmed/28733340 [Accessed January 3, 2019].

Sperling RA, Dickerson BC, Pihlajamaki M, Vannini P, LaViolette PS, Vitolo O V., Hedden T, Becker JA, Rentz DM, Selkoe DJ, Johnson KA (2010) Functional Alterations in Memory Networks in Early Alzheimer's Disease. NeuroMolecular Med 12:27-43 Available at: http://www.ncbi.nlm.nih.gov/pubmed/20069392 [Accessed January 7, 2019].

Spittau B (2017) Aging Microglia-Phenotypes, Functions and Implications for Age-Related Neurodegenerative Diseases. Front Aging Neurosci 9:194 Available at: http://www.ncbi.nlm.nih.gov/pubmed/28659790 [Accessed January 1, 2019].

Srinivasan S, Su M, Ravishankar S, Moore J, Head P, Dixon JB, Vannberg F (2017) TLRexosomes exhibit distinct kinetics and effector function. Sci Rep 7:41623 Available at: http://www.nature.com/articles/srep41623 [Accessed July 24, 2018].

Stanzione R, Bianchi F, Cotugno M, Marchitti S, Forte M, Busceti C, Ryskalin L, Fornai F, Volpe M, Rubattu S (2017) A Decrease of Brain MicroRNA-122 Level Is an Early Marker of Cerebrovascular Disease in the Stroke-Prone Spontaneously Hypertensive Rat. Oxid Med Cell Longev 2017:1-13 Available at:

https://www.hindawi.com/journals/omcl/2017/1206420/ [Accessed August 13, 2017].

Stern DM, Kaiser E, Nawroth PP (1988) Regulation of the Coagulation System by Vascular Endothelial Cells. Pathophysiol Haemost Thromb 18:202-214 Available at: http://www.ncbi.nlm.nih.gov/pubmed/2853122 [Accessed December 16, 2018].

Stevens SL, Ciesielski TM, Marsh BJ, Yang T, Homen DS, Boule J-L, Lessov NS, Simon RP, Stenzel-Poore MP (2008) Toll-Like Receptor 9: A New Target of Ischemic Preconditioning in the Brain. J Cereb Blood Flow Metab 28:1040-1047 Available at: http://journals.sagepub.com/doi/10.1038/sj.jcbfm.9600606 [Accessed January 8, 2019].

Sun B, Dalvi P, Abadjian L, Tang N, Pulliam L (2017) Blood neuron-derived exosomes as 
biomarkers of cognitive impairment in HIV. AIDS 31:F9-F17 Available at: http://www.ncbi.nlm.nih.gov/pubmed/28692534 [Accessed January 5, 2019].

Sun J, Hu H, Ren X, Simpkins JW (2016) Tert-butylhydroquinone compromises survival in murine experimental stroke. Neurotoxicol Teratol 54:15-21 Available at: https://www.sciencedirect.com/science/article/pii/S0892036216300046?via\%3Dihub [Accessed December 12, 2018].

Sun X, Zhang J, Hou Z, Han Q, Zhang C, Tian Z (2015) miR-146a is directly regulated by STAT3 in human hepatocellular carcinoma cells and involved in anti-tumor immune suppression. Cell Cycle 14:243-252 Available at: http://www.ncbi.nlm.nih.gov/pubmed/25607648 [Accessed August 23, 2018].

Swerdlow RH (2018) Mitochondria and Mitochondrial Cascades in Alzheimer's Disease Perry G, Avila J, Tabaton M, Zhu X, eds. J Alzheimer's Dis 62:1403-1416 Available at: http://www.ncbi.nlm.nih.gov/pubmed/29036828 [Accessed November 16, 2018].

Swerdlow RH, Burns JM, Khan SM (2014) The Alzheimer's disease mitochondrial cascade hypothesis: Progress and perspectives. Biochim Biophys Acta - Mol Basis Dis 1842:12191231 Available at: http://www.ncbi.nlm.nih.gov/pubmed/24071439 [Accessed January 7, 2019].

Swerdlow RH, Khan SM (2004) A “mitochondrial cascade hypothesis" for sporadic Alzheimer's disease. Med Hypotheses 63:8-20 Available at: http://www.ncbi.nlm.nih.gov/pubmed/15193340 [Accessed November 28, 2018].

Taanman J-W (1999) The mitochondrial genome: structure, transcription, translation and replication. Biochim Biophys Acta - Bioenerg 1410:103-123 Available at: https://www.sciencedirect.com/science/article/pii/S0005272898001613 [Accessed January 7, 2019].

Taganov KD, Boldin MP, Chang K-J, Baltimore D (2006) NF-B-dependent induction of microRNA miR-146, an inhibitor targeted to signaling proteins of innate immune responses. Available at: www.pnas.orgcgidoi10.1073pnas.0605298103 [Accessed August 9, 2018].

Takahashi A, Okada R, Nagao K, Kawamata Y, Hanyu A, Yoshimoto S, Takasugi M, Watanabe S, Kanemaki MT, Obuse C, Hara E (2017) Exosomes maintain cellular homeostasis by excreting harmful DNA from cells. Nat Commun 8:15287 Available at: http://www.nature.com/doifinder/10.1038/ncomms15287 [Accessed July 24, 2018].

Tang Y-C, Tian H-X, Yi T, Chen H-B (2016) The critical roles of mitophagy in cerebral ischemia. Protein Cell 7:699-713 Available at: http://www.ncbi.nlm.nih.gov/pubmed/27554669 [Accessed January 6, 2019].

Tarkowski E, Andreasen N, Tarkowski A, Blennow K (2003) Intrathecal inflammation precedes development of Alzheimer's disease. J Neurol Neurosurg Psychiatry 74:1200-1205 Available at: http://www.ncbi.nlm.nih.gov/pubmed/12933918 [Accessed November 1, 2018].

Terada H (1990) Uncouplers of oxidative phosphorylation. Environ Health Perspect 87:213-218 Available at: http://www.ncbi.nlm.nih.gov/pubmed/2176586 [Accessed November 1, 2018].

Teri L, Ferretti LE, Gibbons LE, Logsdon RG, McCurry SM, Kukull WA, McCormick WC, Bowen JD, Larson EB (1999) Anxiety of Alzheimer's disease: prevalence, and comorbidity. J Gerontol A Biol Sci Med Sci 54:M348-52 Available at: http://www.ncbi.nlm.nih.gov/pubmed/10462166 [Accessed August 12, 2017]. 
Thal DR, Ghebremedhin E, Orantes M, Wiestler OD (2003) Vascular pathology in Alzheimer disease: correlation of cerebral amyloid angiopathy and arteriosclerosis/lipohyalinosis with cognitive decline. J Neuropathol Exp Neurol 62:1287-1301 Available at: http://www.ncbi.nlm.nih.gov/pubmed/14692704 [Accessed August 24, 2018].

Théry C, Amigorena S, Raposo G, Clayton A (2006) Isolation and Characterization of Exosomes from Cell Culture Supernatants and Biological Fluids. In: Current Protocols in Cell Biology, pp Unit 3.22. Hoboken, NJ, USA: John Wiley \& Sons, Inc. Available at: http://www.ncbi.nlm.nih.gov/pubmed/18228490 [Accessed April 23, 2018].

Théry C, Ostrowski M, Segura E (2009) Membrane vesicles as conveyors of immune responses. Nat Rev Immunol 9:581-593 Available at: http://www.nature.com/articles/nri2567 [Accessed October 29, 2018].

Thompson AG, Gray E, Heman-Ackah SM, Mäger I, Talbot K, Andaloussi S El, Wood MJ, Turner MR (2016) Extracellular vesicles in neurodegenerative disease - pathogenesis to biomarkers. Nat Rev Neurol 12:346-357 Available at: http://www.nature.com/articles/nrneurol.2016.68 [Accessed August 9, 2018].

Thundyil J, Lim K-L (2015) DAMPs and neurodegeneration. Ageing Res Rev 24:17-28 Available at: http://www.ncbi.nlm.nih.gov/pubmed/25462192 [Accessed November 16, 2018].

Ting AT, Bertrand MJM (2016) More to Life than NF-kB in TNFR1 Signaling. Trends Immunol 37:535-545 Available at: http://dx.doi.org/10.1016/j.it.2016.06.002 [Accessed January 7, 2019].

Tkach M, Théry C (2016) Communication by Extracellular Vesicles: Where We Are and Where We Need to Go. Cell 164:1226-1232 Available at: http://dx.doi.org/10.1016/j.cell.2016.01.043 [Accessed May 22, 2018].

Toledano MB, Ghosh D, Trinh F, Leonard WJ (1993) N-terminal DNA-binding domains contribute to differential DNA-binding specificities of NF-kappa B p50 and p65. Mol Cell Biol 13:852-860 Available at: http://www.ncbi.nlm.nih.gov/pubmed/8423807 [Accessed January 2, 2019].

Tolia NH, Joshua-Tor L (2007) Slicer and the Argonautes. Nat Chem Biol 3:36-43 Available at: http://www.nature.com/doifinder/10.1038/nchembio848 [Accessed January 2, 2019].

Tracey D, Klareskog L, Sasso EH, Salfeld JG, Tak PP (2008) Tumor necrosis factor antagonist mechanisms of action: A comprehensive review. Pharmacol Ther 117:244-279 Available at: https://www.sciencedirect.com/science/article/pii/S0163725807002069?via\%3Dihub [Accessed May 24, 2018].

Trajkovic K, Hsu C, Chiantia S, Rajendran L, Wenzel D, Wieland F, Schwille P, Brügger B, Simons M (2007) Ceramide Triggers Budding of Exosome Vesicles into Multivesicular Endosomes. Available at: www.sciencemag.org/cgi/content/full/319/5867/1241/DC1 [Accessed April 20, 2018].

Tuppo EE, Arias HR (2005) The role of inflammation in Alzheimer's disease. Int J Biochem Cell Biol 37:289-305 Available at: http://www.ncbi.nlm.nih.gov/pubmed/15474976 [Accessed August 4, 2017].

Turola E, Furlan R, Bianco F, Matteoli M, Verderio C (2012) Microglial microvesicle secretion and intercellular signaling. Front Physiol 3:149 Available at: http://journal.frontiersin.org/article/10.3389/fphys.2012.00149/abstract [Accessed January 
5, 2019].

Uraoka M, Ikeda K, Kurimoto-Nakano R, Nakagawa Y, Koide M, Akakabe Y, Kitamura Y, Ueyama T, Matoba S, Yamada H, Okigaki M, Matsubara H (2011) Loss of Bcl-2 During the Senescence Exacerbates the Impaired Angiogenic Functions in Endothelial Cells by Deteriorating the Mitochondrial Redox State. Hypertension 58:254-263 Available at: https://www.ahajournals.org/doi/10.1161/HYPERTENSIONAHA.111.176701 [Accessed January 6, 2019].

Uslu S, Akarkarasu ZE, Ozbabalik D, Ozkan S, Çolak O, Demirkan ES, Ozkiris A, Demirustu C, Alatas O (2012) Levels of Amyloid Beta-42, Interleukin-6 and Tumor Necrosis FactorAlpha in Alzheimer's Disease and Vascular Dementia. Neurochem Res 37:1554-1559 Available at: http://www.ncbi.nlm.nih.gov/pubmed/22437436 [Accessed August 12, 2017].

Utsugi-Kobukai S, Fujimaki H, Hotta C, Nakazawa M, Minami M (2003) MHC class I-mediated exogenous antigen presentation by exosomes secreted from immature and mature bone marrow derived dendritic cells. Immunol Lett 89:125-131 Available at: http://www.ncbi.nlm.nih.gov/pubmed/14556969 [Accessed August 2, 2017].

Vader P, Mol EA, Pasterkamp G, Schiffelers RM (2016) Extracellular vesicles for drug delivery. Adv Drug Deliv Rev 106:148-156 Available at: http://www.ncbi.nlm.nih.gov/pubmed/26928656 [Accessed January 6, 2019].

Valadi H, Ekström K, Bossios A, Sjöstrand M, Lee JJ, Lötvall JO (2007) Exosome-mediated transfer of mRNAs and microRNAs is a novel mechanism of genetic exchange between cells. Nat Cell Biol 9:654-659 Available at: http://www.ncbi.nlm.nih.gov/pubmed/17486113 [Accessed August 2, 2017].

van Niel G, D'Angelo G, Raposo G (2018) Shedding light on the cell biology of extracellular vesicles. Nat Rev Mol Cell Biol 19:213-228 Available at: http://www.nature.com/doifinder/10.1038/nrm.2017.125 [Accessed August 9, 2018].

Veerhuis R (2011) Histological and direct evidence for the role of complement in the neuroinflammation of AD. Curr Alzheimer Res 8:34-58 Available at: http://www.ncbi.nlm.nih.gov/pubmed/21143154 [Accessed August 3, 2017].

Vella LJ, Sharples RA, Nisbet RM, Cappai R, Hill AF (2008) The role of exosomes in the processing of proteins associated with neurodegenerative diseases. Eur Biophys J 37:323332 Available at: http://www.ncbi.nlm.nih.gov/pubmed/18064447 [Accessed January 5, 2019].

Verderio C et al. (2012) Myeloid microvesicles are a marker and therapeutic target for neuroinflammation. Ann Neurol 72:610-624 Available at: http://doi.wiley.com/10.1002/ana.23627 [Accessed January 5, 2019].

Wajant H, Pfizenmaier K, Scheurich P (2003) Tumor necrosis factor signaling. Cell Death Differ 10:45-65 Available at: http://www.ncbi.nlm.nih.gov/pubmed/12655295 [Accessed January 2, 2019].

Wang G, Huang Y, Wang L-L, Zhang Y-F, Xu J, Zhou Y, Lourenco GF, Zhang B, Wang Y, Ren R-J, Halliday GM, Chen S-D (2016) MicroRNA-146a suppresses ROCK1 allowing hyperphosphorylation of tau in Alzheimer's disease. Sci Rep 6:26697 Available at: http://www.nature.com/articles/srep26697 [Accessed August 9, 2018].

Wang K, Ye L, Lu H, Chen H, Zhang Y, Huang Y, Zheng JC (2017a) TNF- $\alpha$ promotes extracellular vesicle release in mouse astrocytes through glutaminase. J Neuroinflammation 
14:87 Available at: http://www.ncbi.nlm.nih.gov/pubmed/28427419 [Accessed January 6, 2019].

Wang L, Tang B, Han H, Mao D, Chen J, Zeng Y, Xiong M (2018a) miR-155 Affects

Osteosarcoma MG-63 Cell Autophagy Induced by Adriamycin Through Regulating PTENPI3K/AKT/mTOR Signaling Pathway. Cancer Biother Radiopharm 33:32-38 Available at: http://www.ncbi.nlm.nih.gov/pubmed/29412697 [Accessed January 3, 2019].

Wang P, Guan Y-F, Du H, Zhai Q-W, Su D-F, Miao C-Y (2012) Induction of autophagy contributes to the neuroprotection of nicotinamide phosphoribosyltransferase in cerebral ischemia. Autophagy 8:77-87 Available at: http://www.tandfonline.com/doi/abs/10.4161/auto.8.1.18274 [Accessed January 3, 2019].

Wang P, Hou J, Lin L, Wang C, Liu X, Li D, Ma F, Wang Z, Cao X (2010) Inducible microRNA-155 Feedback Promotes Type I IFN Signaling in Antiviral Innate Immunity by Targeting Suppressor of Cytokine Signaling 1. J Immunol 185 Available at: http://www.jimmunol.org/content/185/10/6226 [Accessed August 4, 2017].

Wang P, Shao B-Z, Deng Z, Chen S, Yue Z, Miao C-Y (2018b) Autophagy in ischemic stroke. Prog Neurobiol 163-164:98-117 Available at: https://www.sciencedirect.com/science/article/pii/S0301008217301442?via\%3Dihub [Accessed January 3, 2019].

Wang Q, Lu Q (2017) Plasma membrane-derived extracellular microvesicles mediate noncanonical intercellular NOTCH signaling. Nat Commun 8:709 Available at: http://www.nature.com/articles/s41467-017-00767-2 [Accessed October 27, 2018].

Wang S, Zhang X, Ju Y, Zhao B, Yan X, Hu J, Shi L, Yang L, Ma Z, Chen L, Liu Y, Duan Z, Chen X, Meng S (2013) MicroRNA-146a Feedback Suppresses T Cell Immune Function by Targeting Stat1 in Patients with Chronic Hepatitis B. J Immunol 191:293-301 Available at: http://www.ncbi.nlm.nih.gov/pubmed/23698745 [Accessed August 21, 2018].

Wang X, Welsh N (2014) Bcl-2 maintains the mitochondrial membrane potential, but fails to affect production of reactive oxygen species and endoplasmic reticulum stress, in sodium palmitate-induced $\beta$-cell death. Ups J Med Sci 119:306-315 Available at: http://www.ncbi.nlm.nih.gov/pubmed/25266628 [Accessed January 6, 2019].

Wang Y, Balaji V, Kaniyappan S, Krüger L, Irsen S, Tepper K, Chandupatla R, Maetzler W, Schneider A, Mandelkow E, Mandelkow E-M (2017b) The release and trans-synaptic transmission of Tau via exosomes. Mol Neurodegener 12:5 Available at: http://molecularneurodegeneration.biomedcentral.com/articles/10.1186/s13024-016-0143-y [Accessed January 5, 2019].

Wei Z, Batagov AO, Schinelli S, Wang J, Wang Y, El Fatimy R, Rabinovsky R, Balaj L, Chen CC, Hochberg F, Carter B, Breakefield XO, Krichevsky AM (2017) Coding and noncoding landscape of extracellular RNA released by human glioma stem cells. Nat Commun 8:1145 Available at: http://www.nature.com/articles/s41467-017-01196-x [Accessed October 28, 2018].

Wekerle H, Linington C, Lassmann H, Meyermann R, Schrader JW, Siers W, Clayberger C, Krensky AW, Burakoff SJ, Reis CS (1986) Cellular immune reactivity within the CNS. Trends Neurosci 9:271-277 Available at: http://linkinghub.elsevier.com/retrieve/pii/0166223686900779 [Accessed August 3, 2017]. Wendeln A-C et al. (2018) Innate immune memory in the brain shapes neurological disease 
hallmarks. Nature 556:332-338 Available at: http://www.nature.com/articles/s41586-0180023-4 [Accessed January 3, 2019].

White IJ, Bailey LM, Aghakhani MR, Moss SE, Futter CE (2006) EGF stimulates annexin 1dependent inward vesiculation in a multivesicular endosome subpopulation. EMBO J 25:112 Available at: http://www.ncbi.nlm.nih.gov/pubmed/16052208 [Accessed August 2, 2017].

Widera D, Mikenberg I, Elvers M, Kaltschmidt C, Kaltschmidt B (2006) Tumor necrosis factor alpha triggers proliferation of adult neural stem cells via IKK/NF-kappaB signaling. BMC Neurosci 7:64 Available at: http://www.ncbi.nlm.nih.gov/pubmed/16987412 [Accessed January 2, 2019].

Willis CM, Ménoret A, Jellison ER, Nicaise AM, Vella AT, Crocker SJ (2017) A Refined BeadFree Method to Identify Astrocytic Exosomes in Primary Glial Cultures and Blood Plasma. Front Neurosci 11:335 Available at: http://www.ncbi.nlm.nih.gov/pubmed/28663721 [Accessed January 5, 2019].

Wilson M, Seymour R, Henderson B (1998) Bacterial Perturbation of Cytokine Networks. Infect Immun 66:2401-2409 Available at: https://iai.asm.org/content/66/6/2401 [Accessed December 16, 2018].

Witwer KW, Buzás EI, Bemis LT, Bora A, Lässer C, Lötvall J, Nolte-’t Hoen EN, Piper MG, Sivaraman S, Skog J, Théry C, Wauben MH, Hochberg F (2013) Standardization of sample collection, isolation and analysis methods in extracellular vesicle research. J Extracell vesicles 2 Available at: http://www.ncbi.nlm.nih.gov/pubmed/24009894 [Accessed January 6, 2019].

Wu D, Cerutti C, Lopez-Ramirez MA, Pryce G, King-Robson J, Simpson JE, van der Pol SM, Hirst MC, de Vries HE, Sharrack B, Baker D, Male DK, Michael GJ, Romero IA (2015) Brain endothelial miR-146a negatively modulates T-cell adhesion through repressing multiple targets to inhibit NF- $\kappa$ B activation. J Cereb Blood Flow Metab 35:412-423 Available at: http://www.ncbi.nlm.nih.gov/pubmed/25515214 [Accessed August 23, 2018].

Wu JP, Kuo JS, Liu YL, Tzeng SF (2000) Tumor necrosis factor-alpha modulates the proliferation of neural progenitors in the subventricular/ventricular zone of adult rat brain. Neurosci Lett 292:203-206 Available at: http://www.ncbi.nlm.nih.gov/pubmed/11018312 [Accessed January 2, 2019].

Wu L-J, Vadakkan KI, Zhuo M (2007) ATP-induced chemotaxis of microglial processes requires P2Y receptor-activated initiation of outward potassium currents. Glia 55:810-821 Available at: http://www.ncbi.nlm.nih.gov/pubmed/17357150 [Accessed August 4, 2017].

Wyss-Coray T (2006) Inflammation in Alzheimer Disease: Driving force, bystander or beneficial response? Nat Med 12:1191-1197 Available at: http://www.ncbi.nlm.nih.gov/pubmed/16960575 [Accessed August 12, 2017].

Wyss-Coray T, Rogers J (2012) Inflammation in Alzheimer disease-a brief review of the basic science and clinical literature. Cold Spring Harb Perspect Med 2:a006346 Available at: http://www.ncbi.nlm.nih.gov/pubmed/22315714 [Accessed November 1, 2018].

Xin H, Wang F, Li Y, Lu Q-E, Cheung WL, Zhang Y, Zhang ZG, Chopp M (2017) Secondary Release of Exosomes From Astrocytes Contributes to the Increase in Neural Plasticity and Improvement of Functional Recovery After Stroke in Rats Treated With Exosomes Harvested From MicroRNA 133b-Overexpressing Multipotent Mesenchymal Stromal Cells. 
Cell Transplant 26:243-257 Available at: http://www.ncbi.nlm.nih.gov/pubmed/27677799 [Accessed January 5, 2019].

Xu B, Zhang Y, Du X-F, Li J, Zi H-X, Bu J-W, Yan Y, Han H, Du J-L (2017) Neurons secrete miR-132-containing exosomes to regulate brain vascular integrity. Cell Res 27:882-897 Available at: http://www.ncbi.nlm.nih.gov/pubmed/28429770 [Accessed August 14, 2017]. Yamada M (2015) Cerebral amyloid angiopathy: emerging concepts. J stroke 17:17-30 Available at: http://www.ncbi.nlm.nih.gov/pubmed/25692104 [Accessed August 10, 2018]. Yamamoto M, Kiyota T, Horiba M, Buescher JL, Walsh SM, Gendelman HE, Ikezu T (2007) Interferon- $\gamma$ and Tumor Necrosis Factor- $\alpha$ Regulate Amyloid- $\beta$ Plaque Deposition and $\beta$ Secretase Expression in Swedish Mutant APP Transgenic Mice. Am J Pathol 170:680-692 Available at: http://www.ncbi.nlm.nih.gov/pubmed/17255335 [Accessed November 1, 2018].

Yang I, Han SJ, Kaur G, Crane C, Parsa AT (2010) The role of microglia in central nervous system immunity and glioma immunology. J Clin Neurosci 17:6-10 Available at: http://www.ncbi.nlm.nih.gov/pubmed/19926287 [Accessed August 24, 2018].

Yang Q, Tong X, Schieb L, Vaughan A, Gillespie C, Wiltz JL, King SC, Odom E, Merritt R, Hong Y, George MG (2017) Vital Signs: Recent Trends in Stroke Death Rates - United States, 2000-2015. MMWR Morb Mortal Wkly Rep 66:933-939 Available at: http://www.cdc.gov/mmwr/volumes/66/wr/mm6635e1.htm [Accessed December 16, 2018].

Yang Y, Boza-Serrano A, Dunning CJR, Clausen BH, Lambertsen KL, Deierborg T (2018) Inflammation leads to distinct populations of extracellular vesicles from microglia. J Neuroinflammation 15:168 Available at: https://jneuroinflammation.biomedcentral.com/articles/10.1186/s12974-018-1204-7 [Accessed January 6, 2019].

Ye J, Jiang Z, Chen X, Liu M, Li J, Liu N (2017) The role of autophagy in pro-inflammatory responses of microglia activation via mitochondrial reactive oxygen species in vitro. $\mathrm{J}$ Neurochem 142:215-230 Available at: http://doi.wiley.com/10.1111/jnc.14042 [Accessed January 7,2019$]$.

Yellen G (2018) Fueling thought: Management of glycolysis and oxidative phosphorylation in neuronal metabolism. J Cell Biol 217:2235-2246 Available at: http://www.ncbi.nlm.nih.gov/pubmed/29752396 [Accessed January 5, 2019].

Yin F, Sancheti H, Patil I (2016) Energy metabolism and inflammation in brain aging and Alzheimer's disease. Free Radic Biol Med 100:108-122 Available at: https://www.sciencedirect.com/science/article/pii/S0891584916302167?via\%3Dihub [Accessed August 23, 2018].

Yin H, Song S, Pan X (2017) Knockdown of miR-155 protects microglia against LPS-induced inflammatory injury via targeting RACK1: a novel research for intracranial infection. J Inflamm (Lond) 14:17 Available at: http://www.ncbi.nlm.nih.gov/pubmed/28804270 [Accessed January 6, 2019].

Zaremba J, Losy J (2001) Early TNF-alpha levels correlate with ischaemic stroke severity. Acta Neurol Scand 104:288-295 Available at: http://doi.wiley.com/10.1034/j.16000404.2001.00053.x [Accessed January 3, 2019].

Zeevi N, Pachter J, McCullough LD, Wolfson L, Kuchel GA (2010) The blood-brain barrier: geriatric relevance of a critical brain-body interface. J Am Geriatr Soc 58:1749-1757 
Available at: http://www.ncbi.nlm.nih.gov/pubmed/20863334 [Accessed July 8, 2017].

Zeller JA, Lenz A, Eschenfelder CC, Zunker P, Deuschl G (2005) Platelet-Leukocyte Interaction and Platelet Activation in Acute Stroke With and Without Preceding Infection. Arterioscler Thromb Vasc Biol 25:1519-1523 Available at: http://www.ncbi.nlm.nih.gov/pubmed/15845906 [Accessed December 16, 2018].

Zhang L, Dong L-Y, Li Y-J, Hong Z, Wei W-S (2012) The microRNA miR-181c controls microglia-mediated neuronal apoptosis by suppressing tumor necrosis factor. $\mathrm{J}$ Neuroinflammation 9 Available at: http://www.jneuroinflammation.com/content/9/1/211 [Accessed August 3, 2017].

Zhao Y, Lukiw WJ (2018) Microbiome-Mediated Upregulation of MicroRNA-146a in Sporadic Alzheimer's Disease. Front Neurol 9:145 Available at: http://www.ncbi.nlm.nih.gov/pubmed/29615954 [Accessed November 25, 2018].

Zhu J, Wang F-L, Wang H-B, Dong N, Zhu X-M, Wu Y, Wang Y-T, Yao Y-M (2017) TNF- $\alpha$ mRNA is negatively regulated by microRNA-181a-5p in maturation of dendritic cells induced by high mobility group box-1 protein. Sci Rep 7:12239 Available at: http://www.nature.com/articles/s41598-017-12492-3 [Accessed January 3, 2019].

Zielinski MR, Dunbrasky DL, Taishi P, Souza G, Krueger JM (2013) Vagotomy attenuates brain cytokines and sleep induced by peripherally administered tumor necrosis factor- $\alpha$ and lipopolysaccharide in mice. Sleep 36:1227-38, 1238A Available at: http://www.ncbi.nlm.nih.gov/pubmed/23904683 [Accessed January 3, 2019].

Zimmermann AK, Loucks FA, Schroeder EK, Bouchard RJ, Tyler KL, Linseman DA (2007) Glutathione binding to the Bcl-2 homology-3 domain groove: a molecular basis for Bcl-2 antioxidant function at mitochondria. J Biol Chem 282:29296-29304 Available at: http://www.ncbi.nlm.nih.gov/pubmed/17690097 [Accessed January 6, 2019].

Zuliani G, Ranzini M, Guerra G, Rossi L, Munari MR, Zurlo A, Volpato S, Atti AR, Blè A, Fellin R (2007) Plasma cytokines profile in older subjects with late onset Alzheimer's disease or vascular dementia. J Psychiatr Res 41:686-693 Available at: https://www.sciencedirect.com/science/article/pii/S0022395606000525?via\%3Dihub [Accessed November 1, 2018]. 